
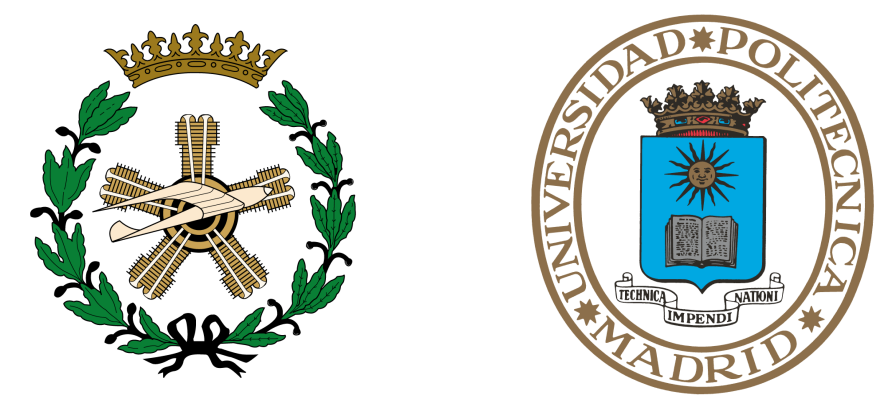

Universidad Politécnica de Madrid

Escuela Técnica Superior de Ingenieros Aeronáuticos

\title{
COMPARACIÓN DE UN NUEVO MÉTODO DE ACELERACIÓN DE ANÁLISIS DINÁMICOS CON MÉTODOS DE REDUCCIÓN DE MODELOS
}

Tesis Doctoral

\section{Juan García Martínez}

Ingeniero Aeronáutico y Licenciado en Matemáticas 

Departamento de Vehículos Aeroespaciales

Escuela Técnica Superior de Ingenieros Aeronáuticos

\title{
Comparación de un nuevo método de aceleración de análisis dinámicos con métodos de reducción de modelos
}

\author{
Tesis Doctoral \\ Juan García Martínez \\ Ingeniero Aeronáutico y Licenciado en Matemáticas
}

Director de la Tesis Doctoral

Francisco Javier Montáns Leal

Dr. Ingeniero Industrial

Catedrático de Universidad 



\section{TRIBUNAL}

Presidente:

\section{Alberto Fraile de Lerma \\ Dr. Ingeniero Industrial}

Universidad Politécnica de Madrid

Vocal:

\section{Ricardo Atienza Pascual}

Dr. Ingeniero Aeronáutico

Universidad Alfonso X el Sabio

Vocal:

Dña. $\mathbf{M}^{a}$ del Carmen Serna Moreno

Dr. Ingeniero Industrial

Universidad de Castilla-La Mancha

Suplente:

D. Juan José Benito Muñoz

Dr. Ingeniero Industrial

Universidad Nacional de Educación a

Distancia
Vocal:

\section{Fernando Cabrerizo García}

Dr. Ingeniero Aeronáutico

Universidad Alfonso X el Sabio

Secretario:

D. Marcos Latorre Ferrús

Dr. Ingeniero Aeronáutico

Universidad Politécnica de Madrid

Suplente:

D. Miguel Ángel Caminero Torija

Dr. Ingeniero Industrial

Universidad de Castilla-La Mancha 

A Sandra, Nora y Bruno, y a mis padres, Balbina y Juan. 



\section{RESUMEN}

La dinámica estructural estudia la respuesta de una estructura ante cargas o fenómenos variables en el tiempo. En muchos casos, estos fenómenos requieren realizar análisis paramétricos de la estructura considerando una gran cantidad de configuraciones de diseño o modificaciones de la estructura. Estos cambios, ya sean en fases iniciales de diseño o en fases posteriores de rediseño, alteran las propiedades físicas de la estructura y por tanto del modelo empleado para su análisis, cuyo comportamiento dinámico se modifica en consecuencia. Un caso de estudio de este tipo de modificaciones es la supervisión de la integridad estructural, que trata de identificar la presencia de daño estructural y prever el comportamiento de la estructura tras ese daño, como puede ser la variación del comportamiento dinámico de la estructura debida a una delaminación, la aparición o crecimiento de grieta, la debida a la pérdida de pala sufrida por el motor de un avión en vuelo, o la respuesta dinámica de construcciones civiles como puentes o edificios frente a cargas sísmicas.

Si a la complejidad de los análisis dinámicos requeridos en el caso de grandes estructuras se añade la variación de determinados parámetros en busca de una respuesta dinámica determinada o para simular la presencia de daños, resulta necesario la búsqueda de medios de simplificación o aceleración del conjunto de análisis que de otra forma parecen inabordables tanto desde el punto de vista del tiempo de computación, como de la capacidad requerida de almacenamiento y manejo de grandes volúmenes de archivos de datos.

En la presente tesis doctoral se han revisado los métodos de reducción de elementos finitos más habituales para análisis dinámicos de grandes estructuras. Se han comparado los resultados de casos de estudio de los métodos más aptos, para el tipo de estructuras y modificaciones descritas, con los resultados de aplicación de un método de reducción reciente. Entre los primeros están el método de condensación estática de Guyan extendido al caso con amortiguamiento no proporcional y posteriores implementaciones de condensaciones dinámicas en diferentes espacios vectoriales. El método de reducción recientemente presentado se denomina en esta tesis DACMAM (Dynamic Analysis in Complex Modal space Acceleration Method), y consiste en el análisis simplificado que proporciona una solución para la respuesta dinámica de una estructura, calculada en el espacio modal complejo y que admite modificaciones estructurales. El método $D A C M A M$ permite seleccionar un número reducido de grados de libertad significativos para la 
dinámica del fenómeno que se quiere estudiar como son los puntos de aplicación de la carga, localizaciones de los cambios estructurales o puntos donde se quiera conocer la respuesta, de forma que al implementar las modificaciones estructurales, se ejecutan los análisis necesarios sólo de dichos grados de libertad sin pérdida de precisión. El método permite considerar alteraciones de masa, rigidez, amortiguamiento y la adición de nuevos grados de libertad.

Teniendo en cuenta la dimensión del conjunto de ecuaciones a resolver, la parametrización de los análisis no sólo resulta posible, sino que es también manejable y controlable gracias a la sencilla implementación del procedimiento para los códigos habituales de cálculo mediante elementos finitos.

En el presente trabajo se muestra la bondad y eficiencia del método en comparación con algunos de los métodos de reducción de grandes modelos estructurales, verificando las diferencias entre sí de los resultados obtenidos y respecto a la respuesta real de la estructura, y comprobando los medios empleados en ellos tanto en tiempo de ejecución como en tamaño de ficheros electrónicos. La influencia de los diversos factores que se tienen en cuenta permite identificar los límites y capacidades de aplicación del método y su exhaustiva comparación con los otros procedimientos. 


\section{ABSTRACT}

Structural dynamics studies the response of a structure under loads or phenomena which vary over time. In many cases, these phenomena require the use of parametric analyses taking into consideration several design configurations or modifications of the structure. This is a typical need in an engineering office, no matter the structural design is in early or final stages. These changes modify the physical properties of the structure, and therefore, the finite element model to analyse it. A case study, that exemplifies this circumstance, is the structural health monitoring to predict the variation of the dynamical behaviour after damage, such as a delaminated structure, a crack onset or growth, an aircraft that suffers a blade loss event or civil structures (buildings or bridges) under seismic loads.

Not only large structures require complex analyses to appropriately acquire an accurate solution, but also the variation of certain parameters. There is a need to simplify the analytical process, in order to bring CPU time, data files, management of solutions to a reasonable size.

In the current doctoral thesis, the most common finite element reduction methods for large structures are reviewed. Results of case studies are compared between a recently proposed method, herein named DACMAM (Dynamic Analysis in Complex Modal space Acceleration Method), and different condensation methods, namely static or Guyan condensation and dynamic condensation in different vectorial spaces. All these methods are suitable for considering non-classical damping. The reduction method DACMAM consist of a structural modification in the complex modal domain which provides a dynamic response solution for the reduced models. This process allows the selection of a few degrees of freedom that are relevant for the dynamic response of the system. These d.o.f. are the load application points, relevant structural points or points in which it is important to know the response. Consequently, an analysis with structural modifications implies only the calculation of the dynamic response of the selected degrees of freedom added, but with no loss of information. Therefore, mass, stiffness or damping modifications are easily considered as well as new degrees of freedom.

Taking into account the size of the equations to be solved, the parameterization of the dynamic solutions is not only possible, but also manageable and controllable due to the easy implementation of the procedure in the standard finite element solvers.

In this thesis, the proposed reduction method for large structural models is compared with 
other published model order reduction methods. The comparison shows and underlines the efficiency of the new method, and verifies the differences in the response when compared with the response of the full model. The CPU time, the data files and the scope of the parameterization are also addressed. 


\section{Agradecimientos}

Cuando empecé con la idea de realizar la tesis pensaba que era un trabajo bastante unipersonal, pero a medida que ha ido pasado el tiempo no he hecho otra cosa que confirmar, día tras día, que estaba totalmente equivocado. Sin la ayuda, apoyo y consejos de todo el mundo que me ha rodeado en este tiempo dudo que hubiera conseguido finalizarla.

En primer lugar quiero resaltar a mi familia, que han vivido día a día el desarrollo de la tesis, dándome todo su apoyo. Durante este tiempo, que son años, me he casado y he tenido dos hijos: Nora y Bruno. Gracias a ellos y a mi esposa Sandra por su paciencia y amor. No quiero olvidarme de mis padres, Juan y Balbina, que siempre me han apoyado y animado con los estudios. Y otro recuerdo a mis hermanas, sobrinos y a Ana María (la Tata). Al resto de la familia, carnal y política, en especial a Rosa, y a mis amigos, todos ellos muy importantes y numerosos, de lo cual me alegro, les llevo siempre en mi corazón.

Al haber realizado esta tesis mientras desarrollaba mi carrera profesional fuera de la Escuela, en el INTA, no puedo dejar de recordar y dar las gracias a todos aquellos que me han apoyado en mi trabajo con su conocimiento, sus ánimos y dándome tantos buenos pequeños recuerdos de risas y alegrías: Santacruz, Ramón F., Ramón S., San Millán, Rafael, José Miguel, Roberto, Antonio, Gabriel, muchos otros con los que he compartido trabajos y retos, y en especial a Iñaki, con quien he compartido y comparto en el aspecto académico multitud de inquietudes.

La tesis la he realizado en el Departamento de Vehículos Aeroespaciales de la Escuela de Aeronáuticos. Empecé con Jesús López Díez, al que recordaré siempre por su gran conocimiento y por sus chistes, con los que siempre me hacía reír. También quiero darle las gracias a Gregorio López, de quien no me faltó apoyo en ningún momento. El tutor con quien tengo la suerte de entregar la tesis, Paco Montans, ha sido pieza clave para que la tesis llegue a buen puerto y me ha aportado siempre su conocimiento y buenos consejos, mil gracias. No me olvido de Iván Muñoz y del resto de sus compañeros del Área de Mecánica de Medios Continuos y Teoría de Estructuras, con quienes, a pesar de no haber tenido un contacto diario, siempre me han prestado su apoyo si lo necesitaba y me han recibido con una gran sonrisa.

Finalmente quiero mencionar a Paco Herrada, con quien he recorrido este largo camino muy estrechamente y con el que espero tener la suerte de poder compartir proyectos de investigación en el futuro. 



\section{Índice General}

Índice General

Índice de figuras $\quad$ V

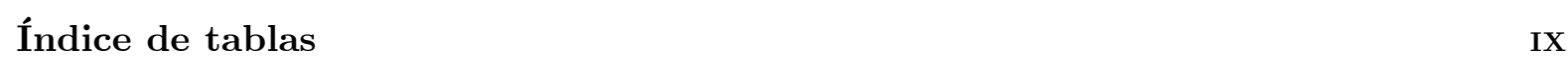

1. INTRODUCCIÓN 1

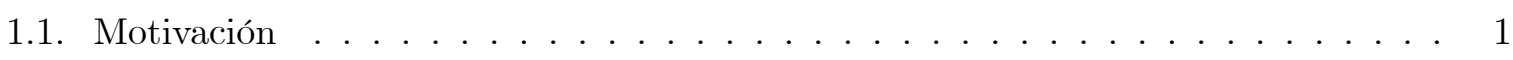

1.2. Parametrización en análisis dinámicos . . . . . . . . . . . 9

1.3. Métodos de reducción de modelos de elementos finitos . . . . . . . . . . 18

1.4. Objetivos y alcance de la tesis . . . . . . . . . . . . . . 25

1.5. Estructura de la tesis . . . . . . . . . . . . . . . . . 27

2. MÉTODO DE ACELERACIÓN DE ANÁLISIS DINÁMICOS EN EL ESPACIO MODAL COMPLEJO (DACMAM) 29

2.1. Descomposición modal . . . . . . . . . . . . . . . . . . . 30

2.2. Introducción de amortiguamiento adicional no proporcional. Nuevo problema de

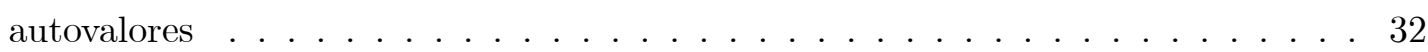

2.3. Adición de masas locales y elementos con rigidez. . . . . . . . . . . . . . . 35

2.4. Grados de libertad adicionales . . . . . . . . . . . . . . . . 36

2.5. Solución del problema general . . . . . . . . . . . . . . . 37

2.6. Ejemplo de aplicación . . . . . . . . . . . . . . . . . 42

3. OTROS MÉTODOS DE REDUCCIÓN DE MODELOS 45 
3.1. Técnicas de reducción consideradas . . . . . . . . . . . . . . . . 45

3.2. Álgebra compleja . . . . . . . . . . . . . . . . . . . . . . . . . . . 47

3.3. Condensación Estática . . . . . . . . . . . . . . . . . . . . 50

3.3.1. Condensación estática en el espacio de los desplazamientos (SCDS) . . . . 50

3.3.2. Condensación estática en el espacio de los estados I (SCSS I) . . . . . . . 52

3.3.3. Condensación estática en el espacio de los estados II (SCSS II) . . . . . . 54

3.4. Condensación Dinámica . . . . . . . . . . . . . . . . . . . . 57

3.4.1. Condensación dinámica en el espacio de los estados I (DCSS I) . . . . . 58

3.4.2. Condensación dinámica en el espacio de los estados II (DCSS II) . . . . 60

3.4.3. Condensación dinámica en el espacio de los estados III (DCSS III) . . . 61

\section{PRIMERAS CONSIDERACIONES RESPECTO A LA COMPARACIÓN} DE LOS MÉTODOS

4.1. Matrices en el espacio físico no simétricas . . . . . . . . . . . . . 65

4.2. Limitaciones del método de aceleración $D A C M A M$ propuesto . . . . . . . . . 67

5. CASO DE ESTUDIO PARA LA COMPARACIÓN DE MÉTODOS DE REDUCCIÓN DE MODELOS

5.1. Descripción del ejemplo . . . . . . . . . . . . . . . . . . . . 71

5.2. Cargas sobre la estructura . . . . . . . . . . . . . . . . . 76

6. COMPARACIÓN DE LOS MÉTODOS 79

6.1. Tiempos de computación . . . . . . . . . . . . . . . . . . 79

6.2. Comparación de autovalores, autovectores y factor de amortiguamiento crítico . . 85

6.3. Parametrizaciones del problema. . . . . . . . . . . . . . . . . . . . . . 101

6.3.1. Parametrización de rigidez del TMD para minimizar desplazamientos . . 112

6.3.2. Parametrización de amortiguamiento del TMD para minimizar aceleraciones . . . . . . . . . . . . . . . . . . . . 117

6.3.3. Parametrización de amortiguamiento del TMD para minimizar desplazamientos . . . . . . . . . . . . . . . . . . . . . . 123

6.3.4. Parametrización de masas del edificio. Búsqueda de respuesta máxima . . 130 
6.3.5. Parametrización de rigideces del edificio. Búsqueda de respuesta máxima . 133

7. CONCLUSIONES

7.1. Otras consideraciones y trabajos futuros . . . . . . . . . . . . . . . 142

Referencias 


\section{Índice de figuras}

1-1. Esquema de análisis. Espacio físico real; Modelo FEM en espacio físico; Modelo en el espacio modal con truncatura de modos. . . . . . . . . . . . . . . . . 3

1-2. Respuesta a carga armónica. Aceleraciones máximas de un nodo del modelo en función de la frecuencia de la carga. . . . . . . . . . . . . . . . 5

1-3. Respuesta a carga transitoria. Desplazamientos de un nodo del modelo en función

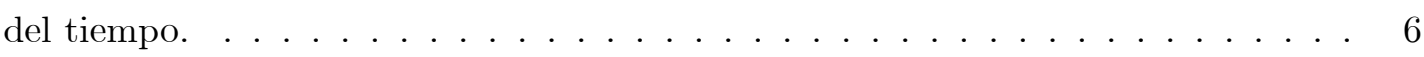

1-4. Flujo de trabajo típico para análisis estáticos y dinámicos. . . . . . . . . . . . 8

2-1. Esquema del método DACMAM: espacio físico, espacio modal real y espacio modal complejo. . . . . . . . . . . . . . . . . . . . 38

2-2. FEM completo del ala de una aeronave no tripulada (cortesía INTA). . . . . . . 43

5-1. Esquema estructural del edificio con 40 plantas y un TMD. . . . . . . . . . . . 72

5-2. Modos propios 1 y 2 del edificio. . . . . . . . . . . . . . . . . . 74

5-3. Modos propios 3 y 4 del edificio. . . . . . . . . . . . . . . . . 74

5-4. Modos propios 5 y 6 del edificio. . . . . . . . . . . . . . . . . 75

5-5. Imagen original del acelerómetro que captó la señal del terremoto de El Centro, 1940. Componentes vertical, N-S, y E-O . . . . . . . . . . . . . 77

5-6. Curva de aceleración del terreno, en función del tiempo, para la componente N-S del terremoto de El Centro, 1940 . . . . . . . . . . . . . . . . . 78

5-7. Transformada discreta de Fourier para la componente N-S de la aceleración del terreno en el terremoto de El Centro, 1940. . . . . . . . . . . . . . . 78 
6-1. Respuesta en aceleración de la planta 5 del edificio a la carga del terremoto. Comparativa de resolución por diversos métodos. . . . . . . . . . . . . . . . . . 105

6-2. Respuesta en aceleración de la planta 40 del edificio a la carga del terremoto. Comparativa de resolución por diversos métodos. . . . . . . . . . . . . . . . . 106

6-3. Respuesta en desplazamientos de la planta 5 del edificio a la carga del terremoto. Comparativa de resolución por diversos métodos. . . . . . . . . . . . . . . . 107

6-4. Respuesta en desplazamientos de la planta 40 del edificio a la carga del terremoto. Comparativa de resolución por diversos métodos. . . . . . . . . . . . . . . . 108

6-5. Respuesta en desplazamientos de la planta 40 del edificio a la carga del terremoto (prolongada en el tiempo con valor nulo para mejorar resultados de la transformada de Fourier). Comparativa de resolución por diversos métodos. . . . 109

6-6. Respuesta en desplazamientos transitoria y permanente a la carga del terremoto para la planta 5 del edificio, evaluando la solución en sumatorio de senos con el método DACMAM . . . . . . . . . . . . . . . . . . . . . . . . . . . . 110

6-7. Respuesta en desplazamientos transitoria y permanente a la carga del terremoto para la planta 40 del edificio, evaluando la solución en sumatorio de senos con el método DACMAM. . . . . . . . . . . . . . . . . . . . . . . . . 110

6-8. Respuesta en aceleraciones transitoria y permanente a la carga del terremoto para la planta 5 del edificio, evaluando la solución en sumatorio de senos con el

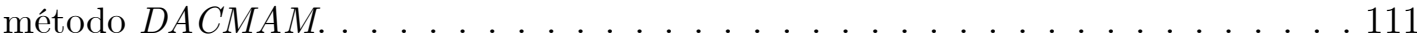

6-9. Respuesta en aceleraciones transitoria y permanente a la carga del terremoto para la planta 40 del edificio, evaluando la solución en sumatorio de senos con el método DACMAM. . . . . . . . . . . . . . . . . . . . . . . . . . . 111

6-10. Resultados de desplazamiento de la planta 40, respuestas en frecuencia. Comparación de métodos: modelo completo en el espacio de lo estados no simétrico y método DACMAM sin truncatura modal. . . . . . . . . . . . . . . . . . . . 113

6-11. Respuesta temporal en desplazamientos de la planta 40 a la carga del terremoto. Caso óptimo de rigidez del TMD para minimizar desplazamientos. Comparación de diversos métodos. . . . . . . . . . . . . . . . . . . . . . 114 
6-12. Respuesta máxima en desplazamientos de la planta 40 en función del parámetro de rigidez del TMD. Comparación de métodos: algoritmo de integración Newmarkbeta, inversa de Fourier con el método DACMAM, inversa de Fourier del modelo completo, y con la condensación DCSS II, caso B (híbrido, con T del SS III). . . 115

6-13. Resultados de aceleración de la planta 40, respuestas en frecuencia. Comparación de métodos: modelo completo en el espacio de lo estados no simétrico y método DACMAM sin truncatura modal. . . . . . . . . . . . . . . . . . . 118

6-14. Respuesta temporal en aceleraciones de la planta 40 a la carga del terremoto. Caso óptimo de amortiguamiento del TMD para minimizar aceleraciones. Comparación de diversos métodos. . . . . . . . . . . . . . . . . . . . . 119

6-15. Respuesta máxima en aceleraciones de la planta 40 en función del parámetro de amortiguamiento del TMD. Algoritmo de integración Newmark-beta. . . . . . . . 120

6-16. Respuesta máxima en aceleraciones de la planta 40 en función del parámetro de amortiguamiento del TMD. Comparación de métodos: algoritmo de integración Newmark-beta, inversa de Fourier con método DACMAM, e inversa de Fourier del modelo completo. . . . . . . . . . . . . . . . . . . . . . . . . . . . 121

6-17. Respuesta máxima en aceleraciones de la planta 40 en función del parámetro de amortiguamiento del TMD. Método DACMAM, combinación SRSS. . . . . . . . 122

6-18. Respuesta máxima en aceleraciones de la planta 40 en función del parámetro de amortiguamiento del TMD. Método DACMAM, combinación TPM. . . . . . . . 122

6-19. Resultados de desplazamiento de la planta 40, respuesta en frecuencia. Comparación de métodos: modelo completo en el espacio de los estados no simétrico y método DACMAM sin truncatura modal. . . . . . . . . . . . . . . . . . . 123

6-20. Respuesta temporal en desplazamientos de la planta 40 a la carga del terremoto. Caso óptimo de amortiguamiento del TMD para minimizar desplazamientos. Comparación de diversos métodos. . . . . . . . . . . . . . . . . . . . . . . . . 124

6-21. Respuesta máxima en desplazamientos de la planta 40 en función del parámetro de amortiguamiento del TMD. Comparación de métodos: algoritmo de integración Newmark-beta, e inversas de Fourier del método DACMAM, del modelo sin reducir y de la condensación DCSS II, caso B (híbrido, con T del SS III). . . 125 
6-22. Respuesta máxima en desplazamientos de la planta 40 en función del parámetro de amortiguamiento del TMD. Método DACMAM, combinación SRSS. . . . . . . 126

6-23. Respuesta máxima en desplazamientos de la planta 40 en función del parámetro de amortiguamiento del TMD. Método DACMAM, combinación TPM. . . . . . . 127

6-24. Esquema de la parametrización de masas en una planta del edificio. . . . . . . . . 130

6-25. Respuesta máxima en desplazamientos de la planta 40 en función del grupo de masas adicionales. Comparación de métodos: algoritmo de integración Newmarkbeta, e inversas de Fourier del método DACMAM, del modelo sin reducir y de la condensación DCSS II, caso B (híbrido, con T del SS III). . . . . . . . . . . . . . 132

6-26. Esquema de la parametrización de rigideces en una planta del edificio. . . . . . . 134

6-27. Respuesta máxima en aceleraciones de la planta 40 en función de grupo de rigideces adicionales consideradas. Algoritmo de integración Newmark-beta. . . . . . . 135

6-28. Respuesta máxima en aceleraciones de la planta 40 en función de grupo de rigideces adicionales consideradas. Método DACMAM. . . . . . . . . . . . . . . 136

6-29. Respuesta máxima en aceleraciones de la planta 40 en función del grupo de rigideces adicionales. Comparación de métodos modales: modelo sin reducir, método DACMAM, y condensación DCSS II, caso B (híbrido, con T del SS III). . . . . . 137 


\section{Índice de tablas}

2.1. Tiempos de computación de los diferentes análisis y modelos del ala de una aeronave no tripulada. . . . . . . . . . . . . . . . . . . . 43

6.1. Descripción del procesador de los equipos empleados en el análisis. . . . . . . . . 80

6.2. Descripción de la memoria de acceso aleatorio de los equipos empleados en el

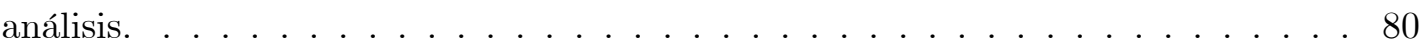

6.3. Tiempos de computación de los diferentes métodos sin reducción. . . . . . . . . . 82

6.4. Tiempos de computación de los diferentes métodos con reducción a 5 grados de libertad. . . . . . . . . . . . . . . . . . . . . . . . 84

6.5. Tiempos de computación de los diferentes métodos con reducción a 10 grados de libertad. . . . . . . . . . . . . . . . . . . . . . . . . 85

6.6. Frecuencias $[\mathrm{rad} / \mathrm{s}]$ con amortiguamiento de los primeros 6 modos. Modelos sin

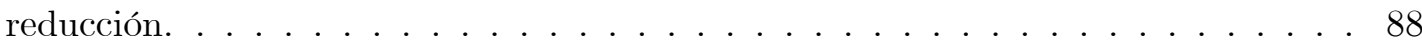

6.7. Errores relativos en porcentaje de las frecuencias de los primeros 6 modos comparados con los del espacio de los estados no simétrico sin reducción. Modelos sin reducción. . . . . . . . . . . . . . . . . . . . . . 88

6.8. Factor de amortiguamiento crítico de los primeros 6 modos. Modelos sin reducción. 89

6.9. Errores relativos en porcentaje de los factores de amortiguamiento crítico de los primeros 6 modos comparados con los del espacio de los estados no simétrico sin reducción. Modelos sin reducir. . . . . . . . . . . . . . . . . . . . . . . . 89 
6.10. Errores relativos en porcentaje de los autovectores de los primeros 6 modos comparados con los del espacio de los estados no simétrico sin reducción. Modelos sin reducir. . . . . . . . . . . . . . . . . . . . . . . . . 90

6.11. Frecuencias $[\mathrm{rad} / \mathrm{s}]$ con amortiguamiento de los primeros 5 modos. Modelos reducidos a 5 grados de libertad. . . . . . . . . . . . . . . . . . . . . . . . 91

6.12. Errores relativos en porcentaje de las frecuencias de los primeros 5 modos comparados con los del espacio de los estados no simétrico sin reducción. Modelos reducidos a 5 grados de libertad. . . . . . . . . . . . . . . . . . . . . 91

6.13. Factor de amortiguamiento crítico de los primeros 5 modos. Modelos reducidos a 5 grados de libertad. . . . . . . . . . . . . . . . . . 92

6.14. Errores relativos en porcentaje de los factores de amortiguamiento crítico de los primeros 5 modos comparados con los del espacio de los estados no simétrico sin reducción. Modelos reducidos a 5 grados de libertad. . . . . . . . . . . . . . . . . 92

6.15. Errores relativos en porcentaje de los autovectores de los primeros 5 modos comparados con los del espacio de los estados no simétrico sin reducción. Modelos reducidos a 5 grados de libertad. . . . . . . . . . . . . . . . . . . . . . 93

6.16. Frecuencias $[\mathrm{rad} / \mathrm{s}]$ con amortiguamiento de los modos 1 al 5. Modelos reducidos a 10 grados de libertad. . . . . . . . . . . . . . . . . . . . . . 94

6.17. Frecuencias $[\mathrm{rad} / \mathrm{s}]$ con amortiguamiento de los modos 6 al 10. Modelos reducidos a 10 grados de libertad. . . . . . . . . . . . . . . . . . . . . 94

6.18. Errores relativos en porcentaje de las frecuencias de los modos 1 al 5 comparados con los del espacio de los estados no simétrico sin reducción. Modelos reducidos a 10 grados de libertad. . . . . . . . . . . . . . . . . . . 95

6.19. Errores relativos en porcentaje de las frecuencias de los modos 6 al 10 comparados con los del espacio de los estados no simétrico sin reducción. Modelos reducidos a 10 grados de libertad.

6.20. Factor de amortiguamiento crítico de los modos 1 al 5 . Modelos reducidos a 10 grados de libertad. . . . . . . . . . . . . . . . . . . 96

6.21. Factor de amortiguamiento crítico de los modos 6 al 10. Modelos reducidos a 10 grados de libertad. 
6.22. Errores relativos en porcentaje de los factores de amortiguamiento crítico de los modos 1 al 5 comparados con los del espacio de los estados no simétrico sin reducción. Modelos reducidos a 10 grados de libertad. . . . . . . . . . . . . . . . 97

6.23. Errores relativos en porcentaje de los factores de amortiguamiento crítico de los modos 6 al 10 comparados con los del espacio de los estados no simétrico sin reducción. Modelos reducidos a 10 grados de libertad. . . . . . . . . . . . . . . 97

6.24. Errores relativos en porcentaje de los autovectores de los modos 1 al 5 comparados con los del espacio de los estados no simétrico sin reducción. Modelos reducidos a 10 grados de libertad. . . . . . . . . . . . . . . . . . . . 98

6.25. Errores relativos en porcentaje de los autovectores de los modos 6 al 10 comparados con los del espacio de los estados no simétrico sin reducción. Modelos reducidos a 10 grados de libertad. . . . . . . . . . . . . . . . . . . . . . . 98

6.26. Valores máximos de desplazamientos y aceleraciones obtenidos por métodos de combinación de análisis espectral. . . . . . . . . . . . . . . . . . . . . . . . . . 103

6.27. Valores óptimos de amortiguamiento del TMD para minimizar la respuesta en aceleraciones ante la carga del terremoto. . . . . . . . . . . . . . . . . . . . . 115

6.28. Tiempos de computación de los diferentes métodos para el caso de parametrización del amortiguamiento del TMD en busca de minimizar aceleraciones. . . 116

6.29. Valores óptimos de amortiguamiento del TMD para minimizar la respuesta en aceleraciones ante la carga del terremoto. . . . . . . . . . . . . . . . . . . 120

6.30. Tiempos de computación de los diferentes métodos para el caso de parametrización del amortiguamiento del TMD en busca de minimizar aceleraciones. . . 121

6.31. Valores óptimos de amortiguamiento del TMD para minimizar la respuesta en desplazamientos ante la carga del terremoto. . . . . . . . . . . . . . . . . . . . 128

6.32. Tiempos de computación de los diferentes métodos para el caso de parametrización del amortiguamiento del TMD en busca de minimizar desplazamiento. . 129

6.33. Tiempos de computación de los diferentes métodos para el caso de parametrización de masas adicionales en las últimas plantas del edificio. . . . . . . . . . 133

6.34. Tiempos de computación de los diferentes métodos para el caso de parametrización de rigideces adicionales en las plantas inferiores del edificio. . . . . . . . 137 


\section{Capítulo 1}

\section{INTRODUCCIÓN}

\subsection{Motivación}

Existen en la naturaleza numerosos fenómenos que pueden describirse adecuadamente sólo si se tienen en cuenta sus efectos a lo largo de un periodo de tiempo. La magnitud temporal determina en la mayoría de los casos el tipo de problema al que nos enfrentamos cuando comparamos las ecuaciones que lo describen en contraposición con los llamados análisis estáticos (Bathe [1996]).

Tradicionalmente se ha entendido por análisis dinámico de estructuras (Chopra [1995]) al análisis de las pequeñas oscilaciones o vibraciones que puede sufrir una estructura alrededor de su posición de equilibrio. En algunos casos dicho análisis es relevante porque ese movimiento oscilatorio produce una modificación de las tensiones y deformaciones existentes. Como resultado de una perturbación exterior un edificio o estructura resistente que bajo la acción de unas cargas estaba en reposo, experimenta oscilaciones que en primera aproximación pueden representarse como un movimiento armónico compuesto, caracterizado por un sistema de ecuaciones lineal (Den Hartog [1984])

Sin embargo, en muchos casos las perturbaciones no tienen por qué ser pequeñas ni la situación de la estructura próxima al equilibrio. Desde el punto de vista de la solución del problema, la primera distinción respecto a los análisis estáticos es la consideración de las fuerzas de inercia, que no pueden despreciarse si la estructura está sometida a aceleraciones. El comportamiento de la estructura está caracterizado por el movimiento provocado por dichas acelera- 
ciones, por lo que habitualmente se le llama respuesta del sistema a las cargas. Las excitaciones generales pueden tener la forma de desplazamientos y velocidades iniciales o fuerzas aplicadas (Paz [1992]). En el primer caso, la respuesta se conoce como respuesta libre o vibración libre y en el segundo, respuesta forzada o vibración forzada. Otro aspecto que diferencia la respuesta dinámica de la estática es la influencia del amortiguamiento. Todas las estructuras reales tienen alguna forma de amortiguamiento que produce la reducción paulatina de las vibraciones libres. Este amortiguamiento, que en muchos casos de aplicación se puede considerar muy pequeño, o bien se desprecia, tomando el nombre de vibraciones no amortiguadas, o bien se considera, denominándose entonces vibraciones amortiguadas. La ecuación de equilibrio dinámico con estas consideraciones queda de la forma (Montáns y Muñoz [2013]):

$$
\left\{\begin{array}{l}
\mathbf{M} \ddot{\mathbf{x}}(t)+\mathbf{C} \dot{\mathbf{x}}(t)+\mathbf{K} \mathbf{x}(t)=\mathbf{F}(t) \\
\mathbf{x}(t=0)=\mathbf{x}_{0} \quad, \quad \dot{\mathbf{x}}(t=0)=\dot{\mathbf{x}}_{0}
\end{array}\right.
$$

Según las consideraciones tenidas en cuenta para el análisis dinámico de estructuras, se tienen sucesivos escalones de complejidad en función de las excitaciones y la respuesta que requiera el análisis.

Es habitual clasificar de diversas maneras los tipos de análisis dinámicos en función del tipo de excitación, el número de grados de libertad o la consideración de amortiguamiento. Para la presente tesis se empleará en general la nomenclatura habitual en el campo de elementos finitos (MSC.Nastran Dynamics [2014]). Con esta nomenclatura, se puede afirmar que los análisis dinámicos más frecuentes son el análisis modal real, el análisis de respuesta en frecuencia y la respuesta transitoria del sistema.

El primer análisis que suele realizarse en dinámica estructural es el análisis modal real (figura 1-1), que consiste en el cálculo de frecuencias y modos propios de la estructura considerada sin amortiguamiento, es decir, determinar las frecuencias y modos según los cuales la estructura tiende a vibrar en respuesta libre tras una perturbación de las condiciones de equilibrio. Los resultados de este análisis de autovalores no tienen en cuenta ninguna carga aplicada, pero se pueden usar para predecir los efectos de diversas excitaciones mediante la superposición modal (basado en el teorema de expansión del álgebra lineal), por lo que caracteriza el comportamiento o respuesta de la estructura bajo cargas dinámicas. Permite conocer la interacción entre un 
componente y sus condiciones de contorno, evaluar cambios en el diseño, realizar diferentes tipos de análisis o correlacionar los resultados numéricos con los experimentales (MSC.Nastran Dynamics [2014]; Harris et al. [2002]). Sin embargo, el número de variables del modelo de elementos finitos suele ser mucho mayor que las variables que pueden verificarse por ensayo, uno de los motivos que hace conveniente la reducción del modelo.

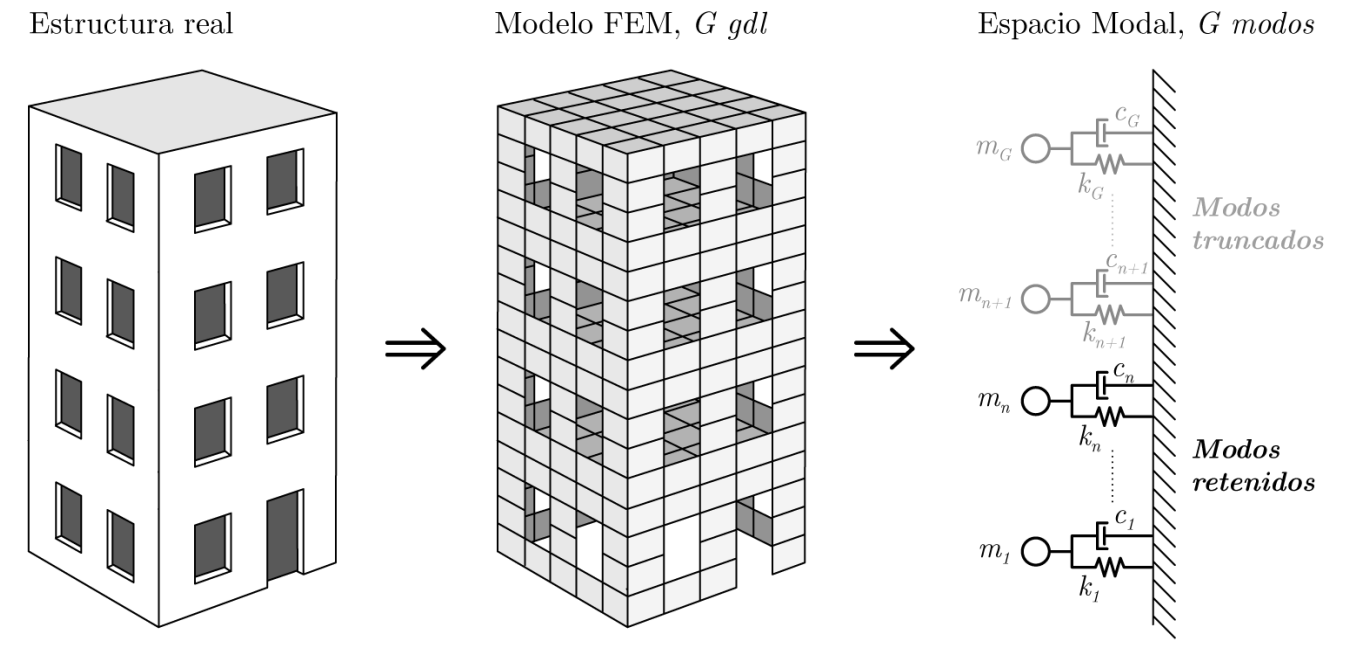

Figura 1-1: Esquema de análisis. Espacio físico real; Modelo FEM en espacio físico; Modelo en el espacio modal con truncatura de modos.

Tras el análisis modal y la extracción de autovalores y vectores propios se emplea el teorema de expansión, que dice que cualquier vector G-dimensional puede ser expresado como combinación lineal de vectores G-dimensionales que sean ortogonales, y por tanto linealmente independientes. Este sistema de vectores forma una base en el espacio modal. Desde el punto de vista de espacios vectoriales, la truncatura modal se puede ver como una herramienta para aproximar la solución exacta si el subespacio que definen los vectores o modos retenidos contiene las soluciones buscadas. Aunque en la realidad las soluciones no se encuentran contenidas en dicho subespacio, sí se puede considerar en muchos casos dicha hipótesis como buena y recomendable al mantenerse los errores resultantes dentro de un rango aceptable. De hecho es la práctica habitual siempre que se recurre al análisis modal en grandes estructuras con muchos 
grados de libertad.

Aplicando el método de la superposición modal (Rayleigh [1945]), la solución de la ecuación dinámica, tras la resolución de un problema de autovalores, puede representarse como la suma de la serie de los modos de vibración de la estructura. En gran parte de los problemas, cuyo rango de frecuencias de interés es bajo, basta con considerar los primeros modos de la serie (Rouleau et al. [2014]). Aunque hay técnicas para corregir el error bajo ciertas condiciones, esto causa que la influencia de modos de altas frecuencias no sea considerada. Es frecuente que los autovalores obtenidos por este método se encuentren en el espacio de números reales ya que, como se ha indicado, se suele considerar, o bien que el amortiguamiento es despreciable, o bien que es aceptablemente modelado por valores proporcionales a la rigidez y masa del sistema o sus inversas (Rayleigh [1945]; Caughey [1960]; Caughey y O'Kelly [1965]). En caso contrario debe resolverse el problema complejo de autovalores (en la tesis se trata este caso en más profundidad).

El análisis de respuesta en frecuencia (figura 1-2) sirve para encontrar la respuesta permanente a una excitación sinusoidal conocida (Shabana [1996]). En la práctica cualquier excitación periódica puede ser representada por una suma de señales sinusoidales al aplicarle la descomposición en serie de Fourier, resultando este tipo de análisis el más indicado para cargas cíclicas. Este tipo de análisis se realiza habitualmente por medio de superposición modal teniendo en cuenta los modos contenidos en el rango de frecuencias de estudio y un número adicional de modos cuya frecuencia es próxima a este rango. También es posible realizar estos análisis por medio de métodos numéricos que integran en el tiempo las variables del sistema. El análisis ante una carga aleatoria definida por un espectro de cargas en frecuencias es un caso particular de los análisis de respuesta en frecuencia.

El último tipo de análisis más común que se ha mencionado es el de la respuesta transitoria del sistema frente a cargas variables en el tiempo (figura 1-3). Este se puede considerar como el caso más general pero implica el conocimiento explícito de la carga en cada instante de tiempo. Suele resolverse mediante diversos métodos o algoritmos de integración, entre los que destaca el método implícito Newmark-beta por su amplia aceptación y por ser incondicionalmente estable con los parámetros habituales (Montáns y Muñoz [2013]). Hay que señalar que se puede considerar la carga transitoria como una carga que se repite en tiempo infinito, 


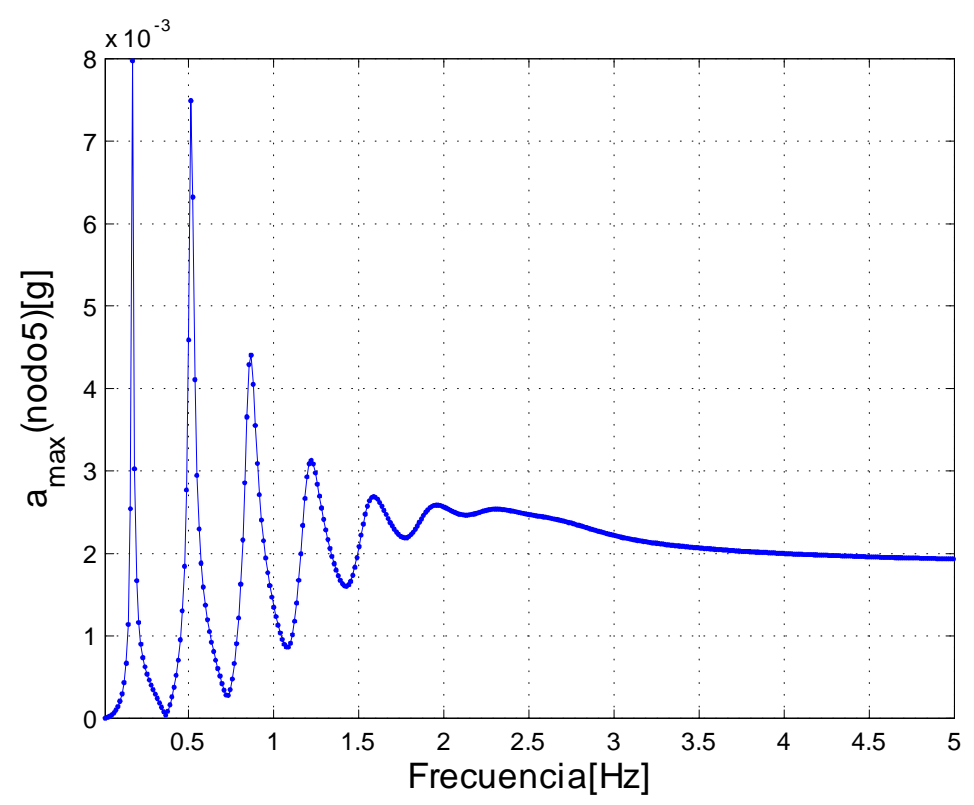

Figura 1-2: Respuesta a carga armónica. Aceleraciones máximas de un nodo del modelo en función de la frecuencia de la carga.

y es ésta la aproximación que se realiza para resolver este tipo de análisis en el dominio de las frecuencias empleando la transformada de Fourier de la carga (en este caso habitualmente hay dos truncaturas: en el número de modos considerados y en el número de términos de la serie de la transformada de Fourier de las cargas aplicadas). El análisis Shock and Response Spectrum (SRS) o análisis espectral es una simplificación de los análisis transitorios para estimar la respuesta dinámica máxima de la estructura ante cargas variables en el tiempo.

Generalmente, los sistemas reales son continuos, al igual que la variación de sus parámetros, aunque en muchos casos es posible simplificar los análisis representando los parámetros distribuidos del sistema por medio de parámetros discretos. De esta forma, los modelos numéricos de estructuras se pueden distinguir entre modelos continuos y modelos discretos o de masas localizadas. En este concepto se basa el método de los elementos finitos (Argyris y Kelsey [1954,1955]; Turner et al. [1956]; Clough [1960]), que permite representar las estructuras reales con modelos de un número finito de grados de libertad, que simbolizan generalmente los desplazamientos en ciertos puntos llamados nudos o nodos. 


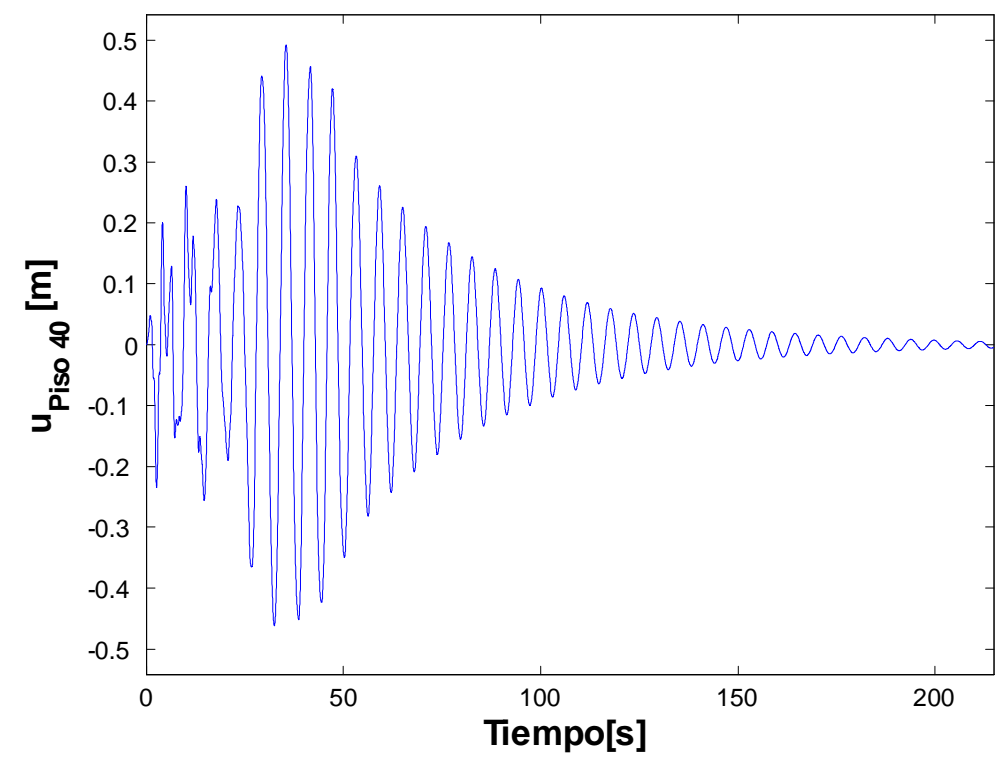

Figura 1-3: Respuesta a carga transitoria. Desplazamientos de un nodo del modelo en función del tiempo.

Hoy en día, este método se emplea cada vez más, evolucionando tanto en precisión como en complejidad de los modelos (Mourelatos et al. [2012]), apoyado por la evolución de los sistemas informáticos y el software comercial que lo sustenta. De esta forma, los modelos actualmente manejados en ingeniería eran impensables hace unos pocos años (Garatani [1999]).

Los modelos de elementos finitos pueden depender del tipo de análisis para el que son desarrollados, aunque en muchos casos se emplea el mismo modelo para todo el análisis estructural, por lo que deben simular adecuadamente el comportamiento de la estructura frente a los diversos casos de carga que vaya a soportar durante su vida de servicio. Un método habitual (MSC.Nastran Dynamics [2014]) es realizar en primer lugar los análisis estáticos necesarios, a continuación el análisis modal y finalizar con los análisis de respuesta forzada (figura 1-4).

El análisis estructural mediante elementos finitos presenta una desventaja que ya se ha citado: dependiendo de la estructura y el detalle que se requiera, los modelos de elementos finitos pueden llegar a ser muy grandes, de millones de grados de libertad. Este tipo de modelos requieren volúmenes de datos de gigabytes y un tratamiento específico que puede implicar 
costes elevados debidos a diversas causas: logísticas, disponibilidad de hardware, limitaciones del software, etc. Evidentemente puede escalarse la potencia de cálculo y de almacenamiento de los sistemas informáticos empleados (Chen et al. [2011]), pero hay que tener en cuenta que el incremento de tiempo empleado en los análisis también impacta en el coste debido al uso de las licencias de los programas de análisis así como del mantenimiento y gestión de las máquinas empleadas.

Un modo de enfrentarse a dicho obstáculo es la simplificación de los análisis o del propio modelo. La simplificación de modelos es una solución muy recomendable en los primeros análisis, especialmente en la fase de diseño preliminar de estructuras, pero debe emplearse con la debida cautela para análisis detallados, ya que tiene limitaciones en función de los métodos empleados.

En base a lo comentado surge la necesidad de acelerar los análisis dinámicos en estos casos, bien sea mediante la reducción del número de variables o por la simplificación de la ejecución, siempre que los resultados se mantengan aproximados, con la precisión requerida, a los de la estructura real ( $\mathrm{Qu}[2004])$. 


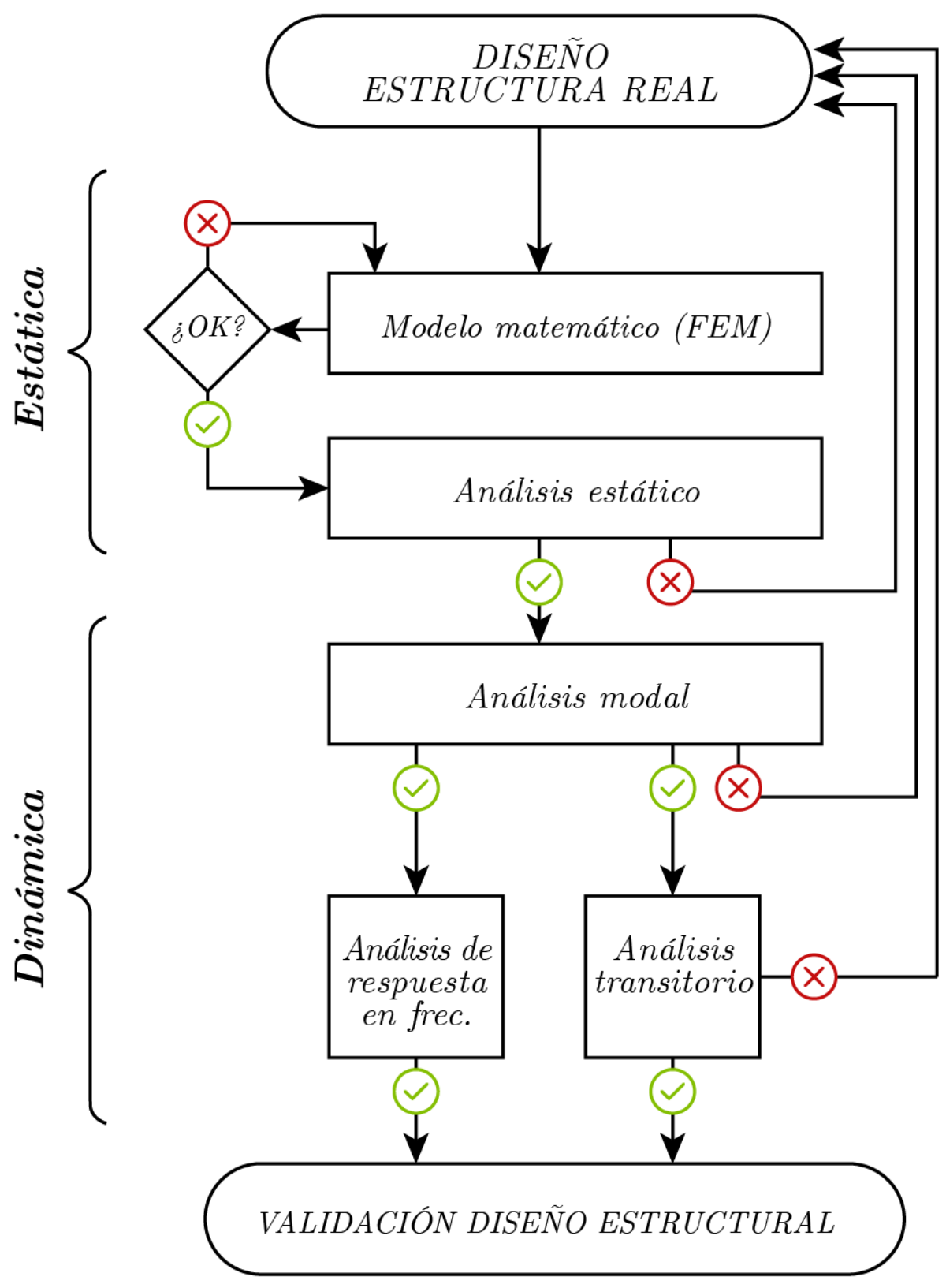

Figura 1-4: Flujo de trabajo típico para análisis estáticos y dinámicos. 


\subsection{Parametrización en análisis dinámicos}

En muchos casos, es interesante conocer la respuesta dinámica de la estructura teniendo en cuenta determinadas variaciones de algún parámetro del sistema. Estos análisis paramétricos suponen la repetición del tipo de análisis necesario, lo que implica que se complique el problema, especialmente en modelos de gran tamaño. Este tipo de análisis es utilizado en el prediseño de estructuras así como en los casos de modificaciones estructurales al estudiar rangos completos de soluciones válidas entre las que seleccionar el conjunto óptimo de un grupo de variables (Wallack et al. [1989]) o modificaciones estructurales que simulan características reales sobre la estructura para conocer la respuesta del sistema o comprobar que se encuentra dentro de los rangos admisibles (Lepidi et al. [2007]).

En el caso concreto de las respuestas dinámicas, hay una gran diversidad de fenómenos que influyen en el estudio del problema, en particular debe considerarse toda variación temporal de cualquier propiedad como la rigidez o la masa. Respecto a la sensibilidad de la respuesta a estas modificaciones, hay que señalar que es muy alta, requiriendo en general que las parametrizaciones que se realizan tengan muchos puntos en el rango de interés, lo que complica enormemente el problema por los tiempos de proceso y volúmenes de datos que implica. Se abordará cómo tratar este problema mediante métodos de reducción en el siguiente apartado.

Un caso de modificación estructural, en este caso en la rigidez, se debe al conocido como daño estructural. Se llama daño a los cambios intencionados o no intencionados en el material y/o en las propiedades geométricas del sistema estructural, incluyendo cambios en las condiciones de contorno o en los elementos de unión del sistema, y que afecta de modo adverso a las funciones de dicho sistema estructural. Todo daño se origina por un defecto o grieta del material, que crecen debido a las condiciones de operación o ambientales a los que está sometido el sistema hasta alcanzar el nivel de daño. El daño implica que haya una pérdida parcial de la funcionalidad del sistema, pero la estructura todavía puede operar satisfactoriamente. Una pérdida total de esa funcionalidad, cuyo estado se denomina fallo, se alcanza o bien tras un proceso de acumulación de daño como entre otros la corrosión o fatiga, o bien tras un suceso propio de la operación del sistema, como sería el aterrizaje en una aeronave, o no programado tal como un terremoto o un impacto.

En los campos aeronáutico y aeroespacial han existido desde sus inicios comerciales exigentes 
normas de seguridad. Una de las consecuencias de estas normas en el campo de la aviación civil son el elevado número y lo costosas que son las revisiones que se deben realizar a las aeronaves periódicamente durante toda su vida útil. Una finalidad de estas revisiones es comprobar el estado estructural de elementos críticos de la estructura cuyo fallo conllevaría una reducción inadmisible de seguridad para la operación de la estructura, lo que podría implicar poner en peligro la vida de personas como sucede en el caso de la aviación comercial.

En el ámbito espacial existe el inconveniente de la dificultad para realizar estas revisiones debido al entorno de operación, con el consiguiente peligro de que el fallo de un elemento del vehículo o satélite conlleve el fallo completo de todo el sistema y los subsiguientes costes que se derivan. Debido a este inconveniente se dimensionan muchos elementos de vehículos espaciales con márgenes de seguridad muy elevados, encareciendo el programa o reduciendo considerablemente la carga de pago útil disponible.

Dentro del campo civil, las condiciones no son menos exigentes, como sucede con el uso continuado bajo condiciones muy diversas de las estructuras, los requisitos de seguridad también aplicables o los problemas logísticos derivados de la clausura temporal debida a daños estructurales para su reparación, como por ejemplo el cierre temporal de un puente por el que circulen decenas de miles de vehículos al día.

El desarrollo de la tecnología en el campo de las inspecciones estructurales y la evaluación de la resistencia residual tras un daño determinado, se ha centrado en los últimos años en el desarrollo de la supervisión de la integridad estructural, SHM (Structural Health Monitoring en inglés), que busca soluciones para los requerimientos cada vez más estrictos de reducir costes de mantenimiento y aumentar al mismo tiempo la seguridad.

SHM es una de las características/funciones de las estructuras inteligentes, que consiste en la continua evaluación de la integridad de la estructura mediante el empleo de sensores (supervisión pasiva) y en algunos casos empleando también actuadores (supervisión activa). Todos ellos van integrados en la estructura, integración que puede realizarse o bien durante el proceso de producción de la estructura o bien a posteriori. La evaluación de la integridad de la estructura se centra en la detección (fiable, sin falsas alarmas), localización y cuantificación (o tamaño) de daños, lo que determina el estado de la estructura. En general se considera la prognosis de vida como la meta principal de esta Tecnología. 
La supervisión de la integridad de una estructura se puede definir (Williamson y Fixter [2006]) como el proceso de detección de daño en una estructura de ingeniería aeroespacial, civil o mecánica. Consiste en la observación del sistema estructural a lo largo del tiempo usando las medidas de la respuesta dinámica del sistema con un muestreo periódico, la extracción de estas medidas de las características del sistema sensibles al daño, y el análisis estadístico de estas características para determinar el estado de la estructura. Si la supervisión es de larga duración, como es el caso de estructuras civiles, se debe actualizar la información de forma periódica para tener en cuenta la degradación producida en la estructura y que el sistema SHM realice la función deseada. En el caso de sucesos como terremotos, impactos, etc., el sistema debe suministrar información fiable de la integridad de la estructura en tiempo real.

Algunos autores (Basseville [2005]) dividen el proceso de establecer un sistema de supervisión de la integridad de una estructura (SHM) en 4 pasos:

- Evaluación del escenario de operación. En este paso se razonan los requisitos y la justificación del empleo de SHM, se establecen los objetivos de daño a encontrar y de las condiciones ambientales o de operación en las que encontrarlo, y se estudian las limitaciones para la adquisición de datos. Hay diversas razones que justifican el empleo del SHM, como pueden ser la necesidad de una herramienta fiable para la detección de daños antes de que produzcan un fallo crítico de la estructura, lo que conllevaría peligro de pérdida de vidas humanas o pérdida de la usabilidad del sistema con la consiguiente pérdida monetaria. Se puede emplear SHM como sustitución de técnicas NDE (Non Destructive Evaluation) que requieren elevados tiempos y costes de personal. También es útil para detectar daños no visibles, como pueden ser zonas no accesibles para la aplicación de técnicas NDE, o también como sucede con los BVID (Barely Visible Impact Damage), muy comunes en materiales compuestos como consecuencia de impactos de pájaros en aeronaves, caídas de herramientas durante labores de mantenimiento, daños por proyectiles en vehículos de combate, etc. Otra posibilidad que ofrece es la de notificar al usuario del sistema estructural la existencia de un daño de modo que el usuario tome las medidas adecuadas para preservar la integridad estructural del sistema hasta que el daño sea reparado, y de este modo poder reducir la severidad de la reparación tanto en cuantía monetaria como en tiempo de parada del servicio que prestase el sistema estructural, o 
incluso evitar la posibilidad de progresar hasta un daño irreversible para la estructura.

- Adquisición y tratamiento previo de los datos. Tras el primer paso se evalúa el tipo de sensores adecuado para la aplicación, el número y su localización, y actuadores si el método elegido lo requiere. También se determina el hardware necesario para la adquisición y almacenaje de los datos. La frecuencia de muestreo máxima también queda definida en este paso. El factor económico puede influir en la selección de estos elementos de manera muy decisiva. Se normalizan los datos con el fin de diferenciar la influencia en los datos de las condiciones ambientales o de operación de la influencia de un daño; una opción muy empleada es usar las señales de excitación de la estructura, cuando estén disponibles, para esta normalización. También se considera en este paso la utilización de filtros para los datos adquiridos e incluso la posibilidad de eliminar series de datos erróneas (e.g.: un sensor roto o mal cableado). Hay que tener en cuenta que para supervisiones durante tiempos largos, las condiciones ambientales y/o de operación pueden cambiar ampliamente, de modo que afecte a la capacidad del sistema SHM para diferenciar en los datos la influencia ambiental/operacional de un daño. El tratamiento de los datos puede ser revisado durante la aplicación del sistema SHM, como puede ser la aplicación de diferentes filtros para diferentes momentos de la adquisición.

- Selección de parámetros característicos del estado estructural y reducción de los datos. En general es necesario reducir la enorme cantidad de datos adquiridos a una parte representativa del estado de la estructura de modo que obtenemos los parámetros característicos para el problema de la detección de daño (en la bibliografía en inglés se denominan distinguishing features). Hay diversas metodologías para realizar esta reducción y la capacidad del sistema para localizar el daño puede depender de una adecuada elección, ya que se podrían elegir parámetros del estado estructural que no fueran sensibles a la aparición de un tipo determinado de daño o un daño localizado en una zona determinada de la estructura. Esta reducción de los datos debe ser robusta, de modo que mantenga las propiedades de sensibilidad (tamaño mínimo de daño que es capaz de detectar el sistema SHM) y resolución (capacidad del sistema SHM para diferenciar dos daños muy próximos) deseadas. 
- Desarrollo del modelo estadístico de confirmación del daño. Esta parte del proceso del SHM consiste en el desarrollo de modelos estadísticos para la diferenciación entre el estado con daño y estado sano o sin daño del sistema estructural. Se pueden dividir estos modelos en dos grandes grupos: Aprendizaje con supervisión (supervised learning) si los algoritmos usados disponen de datos tanto de la estructura sin daño como de la estructura con daño, y aprendizaje sin supervisión (unsupervised learning, o también novelty detection o anomaly detection) si los algoritmos que se emplean no disponen de datos correspondientes a la estructura con daño. Todos ellos emplean análisis estadísticos de las distribuciones de los datos obtenidos con el fin de mejorar la detección del daño.

En la bibliografía se distinguen cuatro o cinco niveles de verificación del daño según los autores Farrar et al. [2006] y Takeda [2006], y que son la meta de todo sistema SHM:

1. Detección del daño.

2. Localización del daño.

3. Clasificación del tipo de daño.

4. Severidad o tamaño del daño.

5. Prognosis de vida.

Queda claro que cada nivel requiere tener los datos de todos los anteriores. También se puede deducir que para que este tipo de sistemas sean eficaces, requieren del uso de modelos de elementos finitos de gran detalle, para los que será necesario emplear métodos de reducción con el fin de obtener tiempos de computación razonables.

Los métodos de aprendizaje sin supervisión no tienen capacidad para estimar el tipo y tamaño del daño.

La clasificación del tipo de daño es el nivel que ciertos autores omiten, pero puede ser un nivel cuya información sea necesaria para verificar los niveles 4 y 5 .

Prognosis de daño es la estimación del remanente de vida útil de una estructura en presencia de daños. Esta estimación se hace en base a los resultados de modelos predictivos que tienen en cuenta: 
- los datos de supervisión y los resultados de SHM de dicha estructura .

- las condiciones ambientales o de operación a que está, ha estado y se prevé que esté sometida la estructura.

- diseño de la estructura, y resultados de análisis detallados y ensayos a nivel de componente y de sistema completo.

En la actualidad los esfuerzos de investigación en sistemas SHM se centran en la prognosis de vida como herramienta clave para el salto a la industria de los sistemas SHM. Por esto es básico poder disponer de métodos de aceleración de análisis dinámicos que colaboren en esta tarea.

Dentro de la supervisión de la integridad estructural hay diversas líneas de investigación atendiendo a las diferentes opciones que brinda la tecnología en cada uno de los pasos necesarios para llegar a la toma de decisión acerca del estado de la estructura. Debido a las fases descritas en la evaluación de la integridad de la estructura, los aspectos de tolerancia al daño y crecimiento de grietas cobran una gran importancia, y sobre ellos se han realizado diferentes trabajos orientados a identificar cómo crecen las grietas y su influencia en la tolerancia al daño en aeronaves con el consiguiente impacto en los análisis estáticos y dinámicos de las estructuras. En Martín de La Escalera et al. [2007] y Valle et al. [2008], los autores Martín de la Escalera, García-Martínez y otros estudian la resistencia residual de paneles metálicos característicos de estructuras de aeronaves, mostrando correlaciones entre resultados experimentales y simulaciones con elementos finitos basadas en el criterio CTOA. Ante el auge del empleo del material compuesto en este tipo de estructuras, se pretendía estudiar la tolerancia al daño en las mismas, como García-Martínez, Armendáriz, San Millán y otros muestran en GarcíaMartínez et al. [2008] y González et al. [2008], donde se recogen correlaciones entre resultados experimentales y simulaciones basadas en métodos energéticos (con algoritmos descritos en el artículo, implementados dentro de software comercial de elementos finitos) del crecimiento de delaminaciones en estructuras de paneles rigidizados fabricados en material compuesto, que modifican las características físicas de los modelos.

También la supervisión de la respuesta en vibración de una estructura es un campo de estudio del análisis estructural enfocado en SHM. La premisa en el uso de métodos de identificación 
de daño para la supervisión de la integridad de una estructura basados en su respuesta en vibración es que, tras el daño, las propiedades mecánicas de la estructura se alteran y por tanto, la respuesta en vibración de la estructura cambia ante las cargas aplicadas externas y/o las condiciones ambientales. Las modificaciones en la respuesta ante cargas estáticas sólo permiten la detección de daño cuando éste está muy avanzado, y por lo tanto no es viable para una detección temprana.

En una estructura, tras sufrir un daño se produce una variación de su rigidez, y debido a la relación directa que hay entre la rigidez, masa y amortiguamiento de una estructura con sus frecuencias propias y modos propios, muchos estudios se han centrado en estudiar la variación de estas propiedades dinámicas de la estructura con el fin de supervisar su integridad estructural desde el punto de vista modal, como en el caso de la actualización o sintonización de modelos de elementos finitos con este fin (García-Hiernaux et al. [2007]). Cabe señalar que la eficacia en los métodos de SHM, entendida como alta sensibilidad ante tamaños de daño muy pequeños, está directamente relacionada con la densidad de puntos empleada en las parametrizaciones durante la fase de aprendizaje, por lo que se vuelven a poner de relieve los altos requisitos en tiempo de computación y volumen de datos que implican este tipo de análisis cuando se busca que tengan alta eficacia.

En el ámbito aeroespacial, otro caso de estudio que produce un importante desequilibrio dinámico y requiere un importante esfuerzo de cálculo paramétrico son los fenómenos relacionados con excitaciones dinámicas severas originadas por fallos en el sistema de propulsión del avión.

Aunque son diversos, pueden citarse, entre otros, el montaje desequilibrado de la hélice o etapas de compresión o turbina, fallo de alguno de sus rodamientos o pérdida de pala. Los sistemas de sujeción de los motores deben diseñarse de forma que absorban estas vibraciones y, en caso necesario, producir la suelta del motor antes de que se puedan producir graves daños en el avión. Es evidente que la simulación de estas modificaciones estructurales conlleva sustanciales modificaciones de los modelos de elementos finitos empleados en la realización de análisis dinámicos. Es necesario considerar una gran cantidad de parámetros involucrados, los autores Armendáriz y García-Martínez tienen en cuenta junto a otros la posición angular de la pala, la velocidad angular, el tamaño de la pala, su rigidez y el amortiguamiento de la estructura 
en los análisis dinámicos en (Armendáriz et al. [2015]) para verificar que las cargas sobre la estructura se mantienen dentro del rango requerido.

Un último caso de aplicación es el relacionado con la respuesta dinámica a fenómenos sísmicos que influye en el diseño y análisis de estructuras civiles (Chopra [1995]; Paz [1992]). Al margen de la complejidad del origen del fenómeno, las excitaciones dinámicas que trasladan a las construcciones se caracterizan por su variedad de contenido en frecuencia. En la tesis se desarrollará un ejemplo de estructura sujeto a este tipo de cargas, de las que se hablará en detalle más adelante. Este tipo de cargas se ha estudiado tradicionalmente con la técnica de los análisis espectrales (también empleada para el estudio de la respuesta de estructuras ante impactos), donde se reduce la estructura de múltiples grados de libertad a la superposición de sistemas de un grado de libertad, cuyo número será el número de modos tenidos en consideración. Esta metodología, propuesta por Housner [1941] para resolver las limitaciones de computación de aquella época tiene como ventajas su sencillez y ser una técnica tradicionalmente empleada en estudios por lo que está implementada en muchos códigos comerciales de elementos finitos para el cálculo de la respuesta dinámica máxima de la estructura. Por contra tiene también limitaciones, entre la que cabe destacar su limitada precisión y que el amortiguamiento que se puede considerar se limita a amortiguamiento modal, que en ciertos casos no simula adecuadamente la realidad. En la tesis se estudiará la respuesta de la estructura mediante un nuevo método de superposición modal en el espacio de los estados complejos (Herrada-Martín [2016]), que permite un amortiguamiento estructural no clásico y se compararán los resultados con una respuesta transitoria obtenida por medio de algoritmos de integración teniendo en consideración varias parametrizaciones.

Todos los ejemplos descritos anteriormente se pueden considerar como casos de uso de la parametrización debido a modificaciones estructurales. En el ámbito del análisis dinámico, se entiende por modificación estructural la técnica por la que cambian las propiedades físicas de una estructura para alterar su comportamiento dinámico (Pomazal y Snyder [1971]; Baldwind y Hutton [1985]; Nad [2007]). Es frecuente que se propongan para la mejora de la respuesta de una estructura existente o para el diseño de una estructura que debe cumplir unas especificaciones ya sean de aceleraciones, velocidades o desplazamientos. También se pueden plantear desde el punto de vista preventivo como un método para predecir las condiciones o modificaciones máximas 
que puede soportar la estructura sin poner en riesgo la seguridad, ya sea por la aparición de un daño que puede crecer como en el caso de grietas o delaminaciones, o por la modificación de rigideces y masas añadidas a la estructura como en el caso de un edificio. Por lo tanto, las modificaciones estructurales se han ido incorporando de diversas maneras al diseño estructural de forma que sus análisis sean precisos y adecuados tanto en tiempo como en necesidad de cálculo (Avitabile [2003]).

Como conclusión se puede extraer que hay numerosas aplicaciones que requieren de parametrizaciones, o bien con un elevado número de parámetros, o bien con una densidad de valores del parámetro considerado muy grande, o incluso ambos al mismo tiempo. La consecuencia de este requisito es otra necesidad, la de equipos con velocidades de proceso altas y capacidad de gestión y almacenaje de volúmenes de datos enorme. Los métodos de aceleración de análisis o reducción de modelos consiguen reducir en parte estas exigencias y constituyen por tanto una herramienta esencial de trabajo en el análisis de estructuras para las aplicaciones descritas. A continuación se describen los empleados con mayor frecuencia en el campo del análisis estructural. 


\subsection{Métodos de reducción de modelos de elementos finitos}

Con el objetivo de obtener las concentraciones de esfuerzos, la predicción de autovalores y autovectores asociados a frecuencias propias, y la respuesta de un sistema en el tiempo o en la frecuencia a una cierta excitación se usan, como se ha comentado, modelos detallados de elementos finitos. Desde hace tiempo, se han empleado diversas técnicas para la reducción de dichos modelos de forma que se evite realizar costosos análisis, especialmente en el caso de prolijas parametrizaciones, de los modelos completos. Se han utilizado en análisis globallocal, reanálisis, optimización, problemas de autovalores, vibraciones y pandeo, estudios de sensibilidad y diseño de parámetros de control y detección de daño (Qu [2004]; Besselink [2013]). Dado que el modelo reducido no retiene todas las características de la estructura completa, el gran objetivo de los métodos de reducción consiste en lograr el modelo más reducido posible con la menor pérdida de información del modelo completo.

La ecuación dinámica de equilibrio tiene la forma:

$$
\mathbf{M} \ddot{\mathbf{x}}(t)+\mathbf{C} \dot{\mathbf{x}}(t)+\mathbf{K x}(t)=\mathbf{F}(t)
$$

donde $\mathbf{M}, \mathbf{C}, \mathbf{K} \in \mathbb{R}^{G \times G}$ son las matrices de masa, amortiguamiento y rigidez del modelo completo y $\mathbf{x}, \dot{\mathbf{x}}, \ddot{\mathbf{x}} \in \mathbb{R}^{G}$ son respectivamente los vectores desplazamiento, velocidad y aceleración de la respuesta frente a cargas exteriores de todos los grados de libertad de la estructura. Si se considera la reducción del modelo mediante una transformación de coordenadas:

$$
\mathbf{x}(t)=\mathbf{T u}(t)
$$

siendo $\mathbf{T} \in \mathbb{R}^{G \times G}$ la matriz de transformación de coordenadas y $\mathbf{u} \in \mathbb{R}^{G}$ las coordenadas del modelo reducido. Si la matriz $\mathbf{T}$ es constante en el tiempo, las derivadas de $\mathbf{x}$ se obtienen de la siguiente manera:

$$
\begin{aligned}
\dot{\mathbf{x}}(t) & =\mathbf{T} \dot{\mathbf{u}}(t) \\
\ddot{\mathbf{x}}(t) & =\mathbf{T} \ddot{\mathbf{u}}(t)
\end{aligned}
$$


Los métodos de reducción de modelos pueden clasificarse según el tipo de variables con las que trabajan: reducción de variables físicas, de variables generalizadas e híbridos.

En el primer tipo, el modelo reducido se obtiene reduciendo las variables físicas del modelo completo. Es la técnica más evidente ya que las variables del modelo reducido forman parte de las variables del modelo completo con el sentido físico que eso conlleva. La reducción de variables físicas suele llamarse condensación estática o dinámica y su matriz de transformación de coordenadas toma la forma siguiente:

$$
\mathbf{T}=\left[\begin{array}{l}
\mathbf{I} \\
\mathbf{R}
\end{array}\right]
$$

siendo I la matriz unidad del orden del sistema reducido: $m$ (que se denominarán grados de libertad maestros o "master" mientras el resto de grados de libertad serán los esclavos o "slave") y $\mathbf{R}$ se denomina habitualmente matriz de condensación. En esta tesis nos referiremos en general a la matriz de condensación dinámica; la llamada condensación estática (Guyan [1965]) ignora los efectos dinámicos en la reducción del modelo y se considera válida solo para un rango determinado de bajas frecuencias entre 0 y la llamada frecuencia de corte. Generalmente este tipo de reducción es computacionalmente más eficiente que las otras dos de variables no físicas, aunque su precisión y convergencia dependen de la selección de los grados de libertad maestros (Kim y Lee [2014]). Se consideran tres tipos de matrices de condensación dinámicas: dependientes de un único modo, de varios modos y dependiente de la respuesta. En el primer caso, la matriz se define como la relación que hay en un autovector entre sus grados de libertad principales y esclavos:

$$
\phi_{s}=\mathbf{R} \phi_{m}
$$

siendo $\phi_{s}$ la partición del autovector de los grados de libertad totales en esclavos y $\phi_{m}$ de los grados de libertad totales en grados principales. Para obtener la matriz de condensación dinámica se aplica la partición a la ecuación de autovalores del modelo completo: 


$$
(\mathbf{K}-\lambda \mathbf{M}) \boldsymbol{\phi}=\mathbf{0} \Rightarrow\left[\begin{array}{cc}
\mathbf{K}_{m m} & \mathbf{K}_{m s} \\
\mathbf{K}_{s m} & \mathbf{K}_{s s}
\end{array}\right]\left[\begin{array}{c}
\boldsymbol{\phi}_{m} \\
\boldsymbol{\phi}_{s}
\end{array}\right]-\lambda\left[\begin{array}{ll}
\mathbf{M}_{m m} & \mathbf{M}_{m s} \\
\mathbf{M}_{s m} & \mathbf{M}_{s s}
\end{array}\right]\left[\begin{array}{c}
\boldsymbol{\phi}_{m} \\
\boldsymbol{\phi}_{s}
\end{array}\right]=\mathbf{0}
$$

De la segunda fila de la ecuación se obtiene

$$
\mathbf{K}_{s m} \boldsymbol{\phi}_{m}+\mathbf{K}_{s s} \boldsymbol{\phi}_{s}-\lambda \mathbf{M}_{s m} \boldsymbol{\phi}_{m}-\lambda \mathbf{M}_{s s} \boldsymbol{\phi}_{s}=\mathbf{0}
$$

y agrupando términos

$$
\left(\mathbf{K}_{s s}-\lambda \mathbf{M}_{s s}\right) \phi_{s}=-\left(\mathbf{K}_{s m}-\lambda \mathbf{M}_{s m}\right) \boldsymbol{\phi}_{m},
$$

de modo que la matriz de condensación dinámica depende del autovalor del modo

$$
\mathbf{R}(\lambda)=-\left(\mathbf{K}_{s s}-\lambda \mathbf{M}_{s s}\right)^{-1}\left(\mathbf{K}_{s m}-\lambda \mathbf{M}_{s m}\right)
$$

De forma análoga, las matrices de condensación dependientes de varios modos (en este caso p) se rigen por:

$$
\boldsymbol{\Phi}_{s p}=\mathbf{R} \boldsymbol{\Phi}_{m p}
$$

y la matriz resulta:

$$
\mathbf{R}=\boldsymbol{\Phi}_{s p} \boldsymbol{\Phi}_{m p}^{T}\left(\boldsymbol{\Phi}_{m p} \boldsymbol{\Phi}_{m p}^{T}\right)^{-1}
$$

Esta matriz de condensación dinámica es la misma que la que proporciona el método del sistema equivalente de reducción-expansión (SEREP) (O'Callahan et al. [1989]).

Por último, la matriz de condensación dependiente de la respuesta se define en base a las relaciones entre las respuestas de los grados de libertad principales y esclavos:

$$
\mathbf{x}_{s}(t)=\mathbf{R} \mathbf{x}_{m}(t)
$$

donde las respuestas del sistema se simulan usando un esquema de integración temporal. 
Todas las coordenadas que no son físicas se denominan habitualmente coordenadas generalizadas. Dos métodos conocidos que usan este tipo de coordenadas son el de coordenadas modales y el de las bases de Ritz (Wilson [1985]). Como se ha comentado, en el primer caso, las respuestas dinámicas de un modelo de $G$ grados de libertad pueden expresarse en función de las coordenadas modales q del espacio modal. Si se tienen en cuenta sólo $m$ coordenadas modales, de las $G$ que definen una transformación de espacios biunívoca, se tiene:

$$
\mathbf{x}=\sum_{i=1}^{G} \phi_{i} q_{i}(t)=\mathbf{\Phi q} \Rightarrow \mathbf{x} \simeq \sum_{i=1}^{m} \phi_{i} q_{i}(t)=\mathbf{\Phi}_{m} \mathbf{q}_{m}
$$

siendo $\mathbf{\Phi}_{m} \in \mathbb{R}^{G \times m}$ la matriz de $m$ autovectores del modelo completo en la que cada columna es un autovector de los $m$ modos considerados en la reducción y $\mathbf{q}_{m}$ el vector columna de las $m$ coordenadas generalizadas correspondientes. Dependiendo de la frecuencia de excitación, los $m$ modos considerados pueden ser los de frecuencias más bajas o los que estén en un rango de frecuencias de interés. Sustituyendo la Ec. (1.15) en la Ec. (1.2) y premultiplicando por la traspuesta de la matriz de autovectores se obtiene:

$$
\overline{\mathbf{M}} \ddot{\mathbf{q}}_{m}+\overline{\mathbf{C}} \dot{\mathbf{q}}_{m}+\overline{\mathbf{K}} \mathbf{q}_{m}=\overline{\mathbf{F}}
$$

donde $\overline{\mathbf{M}}$ es la matriz modal de masas, $\overline{\mathbf{C}}$ es la matriz modal de amortiguamiento y $\overline{\mathbf{K}}$ es la matriz modal de rigidez. La primera y la última son diagonales en modelos lineales. En cambio, la matriz de amortiguamiento puede no serlo dependiendo del tipo de amortiguamiento. Si lo fuera las ecuaciones se desacoplarían y quedaría un sistema de $m$ ecuaciones del espacio modal que puede verse como un sistema de $m$ modelos de un grado de libertad, con los cuales, a través de la superposición de los modos, darían la respuesta en el espacio físico. Ya se ha mencionado que un número limitado de modos de vibración de la estructura puede representar el comportamiento dinámico de la estructura completa a cambio de una pérdida de precisión. La tarea importante del ingeniero de estructuras recae en saber cuándo esa pérdida de precisión es relevante para cada problema concreto. 


$$
\mathbf{x}=\sum_{i=1}^{m} \boldsymbol{\phi}_{i} q_{i}+\underbrace{\sum_{j=m+1}^{G} \phi_{j} q_{j}}_{\text {Truncatura }}
$$

El segundo ejemplo de coordenadas generalizadas que se ha mencionado, los vectores de Ritz, puede aproximar adecuadamente el comportamiento de la estructura si las cargas son conocidas. Esta sustitución puede soslayar el costoso cálculo de autovalores y proporcionar la adecuada precisión en algunos casos ya que puede entenderse como una especie de superposición modal generalizada. La construcción de los vectores de Ritz suele ser computacionalmente más eficiente que los autovectores exactos, aunque la ecuación del movimiento tiene sus componentes acopladas (se trata en resumen de un cambio de coordenadas no ortogonal). El método muestra sus ventajas cuando la resolución del problema requiere el cálculo de un número reducido de autovalores en modelos grandes. También muestra su utilidad para acelerar el proceso de iteración para la búsqueda de autovalores si se eligen adecuadamente, como sugiere Bathe en su método de iteración de subespacios (Bathe [1996]; Montáns y Muñoz [2013]).

El método de la corrección estática y el de aceleración modal (Rixen [2009]) tratan de considerar la contribución de los modos omitidos. En estos casos se añade un término corrector basado en las contribuciones estáticas de los modos no considerados. Una mejora de la anterior técnica es el llamado método del aumento de la truncatura modal que consiste en el uso de la corrección estática como una dirección adicional para la expansión de la truncatura (Rixen [2001]; Tham et al. [2009]). Este vector pasa a formar parte del espacio reducido.

En cualquier caso, en estos métodos es clave la extracción de autovectores y autovalores por el coste computacional que implica su cálculo para grandes estructuras (Sutter et al. [1988]; Bahai et al. [2002]; Majkut [2010]; Wilson [2000]; Lázaro y Pérez-Aparicio [2013]; Lázaro et al. [2012]; Martínez-Aguirre y Elejabarrieta [2011]; Zeng [1995]).

La reducción híbrida es básicamente una combinación de la condensación de Guyan aplicada a los grados de libertad no retenidos y la reducción en el espacio modal aplicada a los grados de libertad retenidos (Kim [1996]). Estos métodos tienen mucho en común con la técnica de la síntesis modal por componentes (Component Modal Synthesis, CMS), que se puede dividir en tres variantes: de interfaz fija (Craig Jr. y Bampton [1968]; Hurty [1965]), de interfaz libre e híbrida, que es una combinación de los anteriores. La primera de las variantes consiste en 
dividir la estructura en componentes, y obtener las matrices del sistema de cada componente por separado bajo la hipótesis de que los puntos de interfaz están fijos en el espacio. Estas matrices se ensamblan dando lugar a un modelo global reducido que es el que se resuelve en coordenadas generalizadas. La solución en coordenadas físicas se obtiene aplicando la matriz de transformación, que en este caso es de la forma:

$$
\mathbf{T}_{C M S}=\left[\begin{array}{cc}
\boldsymbol{\Phi}_{N} & \mathbf{R}_{C} \\
\mathbf{0} & \mathbf{I}
\end{array}\right]
$$

La variante con los grados de libertad de la interfaz considerados como libres tiene la ventaja de que los grados de libertad de la interfaz no aparecen en las ecuaciones de movimiento finales aunque su convergencia es peor (Goldman [1969]; Hou [1969]).

Generalmente, todos los métodos de reducción de modelos se llevan a cabo en un único paso. Sin embargo, se han desarrollado numerosos algoritmos iterativos para mejorar su precisión (Friswell et al. [1995]; Suarez y Singh [1992]; Liu y Wu [2011]) que se basan en la obtención del modelo reducido en un segundo paso que es usado a su vez para la estimación de la matriz de condensación dinámica. En otros casos (Paz [1984]; Paz [1989]), las ecuaciones de la condensación dinámica se derivan directamente pero debido a su no linealidad requieren el uso de esquemas iterativos.

Existen otros métodos de reducción de modelos. Se mencionan por último algunos métodos relevantes que no se han tratado en esta introducción: "Balanced Realization Method" (Moore [1981]) basado en técnicas de control, "Proper Orthogonal Decomposition" (POD) que emplea los valores singulares de las matrices del sistema en lugar de los autovalores (Rathinam y Petzold [2003]), o "Condensation Model Reduction" (CMR) que plantea la reducción con un operador diferencial de segundo orden (Flippen Jr. [1994]).

En la presente tesis doctoral se estudia la aplicación de un método de aceleración de análisis dinámicos en modelos de elementos finitos que mantiene la información de toda la estructura (Allemang y Brown [1987]; Wallack et al. [1988]; Li y He [1999]; O'Callahan [2003]) en ciertos grados de libertad de interés a través de la información contenida en los modos propios del problema. Esto permite, siempre que se seleccionen con el adecuado sentido físico dichos grados de libertad, una elevada reducción en el número de variables manejadas con el consiguiente ahorro 
de tiempo y memoria de ordenador. Esta técnica permite la implementación de modificaciones estructurales (Vibrant Technology [2014]; Schwarz et al. [2007]; Schwarz y Richardson [1997]) no sólo de masa, rigidez sino también de amortiguamiento e incluso la adición de nuevos grados de libertad en el modelo.

Como se ha comentado, se compara dicho método con algunos de los más frecuentemente usados, métodos de condensación estática y condensación dinámica, mostrando su utilidad, eficiencia y flexibilidad en diferentes aplicaciones. 


\subsection{Objetivos y alcance de la tesis}

El objetivo de la presente tesis doctoral es realizar una comparación de métodos de reducción de modelos estructurales aplicados a modelos de elementos finitos de grandes estructuras, sometidas a cargas dinámicas, y de forma que puedan realizarse con precisión adecuada y con eficiencia análisis paramétricos.

En la introducción se ha expuesto con bastante detalle la supervisión de la integridad estructural, campo que corresponde al trabajo realizado en los inicios de esta tesis. Para dicha técnica los análisis dinámicos son una herramienta muy útil, ya que la respuesta dinámica del sistema es en general más sensible a la presencia de pequeños daños que la respuesta estática, y por ello tiene notables ventajas en la detección, localización y cuantificación del daño. Si a dichos análisis dinámicos se les añade parametrizaciones de las propiedades físicas del modelo, las ventajas aumentan, pudiéndose aplicar esta característica desde el entrenamiento de redes neuronales para sistemas con aprendizaje sin supervisión enfocados en la detección de daño, hasta en la prognosis de vida de la estructura con daño.

Para la aplicación concreta al estudio del efecto de modificaciones estructurales locales y paramétricas en la respuesta dinámica, se ha realizado la revisión de los métodos empleados habitualmente en la reducción de modelos de elementos finitos. Entre ellos se han seleccionado varios métodos de condensación de grados de libertad como métodos óptimos para dicha aplicación y se han comparado sus resultados con un método propuesto recientemente (Herrada-Martín [2016]), que consiste en el análisis simplificado de un número reducido de grados de libertad del espacio modal que permite tener en cuenta modificaciones estructurales de masa, rigidez, amortiguamiento y la adición de nuevos grados de libertad. El método ya ha sido implementado partiendo de resultados modales de los softwares ANSYS y MSC.Nastran, y ha probado su validez en un caso práctico de una gran estructura, el ala de una aeronave no tripulada. En la comparación de esta tesis se han considerado la diferencia de los resultados respecto a la respuesta real de la estructura y la eficiencia de cada método en la ejecución de los diversos análisis, empleando como estructura un ejemplo sencillo sobre el que se tiene control total de sus parámetros, y que permite abordar los análisis con los métodos de reducción que se contemplan y con otros métodos sin reducción para la validación de los resultados.

De esta forma se puede conocer el alcance real de cada uno de los métodos estudiados y la 
influencia que suponen los diversos factores que intervienen gracias a su aplicación en casos de estudio iguales. 


\subsection{Estructura de la tesis}

En la introducción, tras presentar la motivación de la tesis doctoral basada en la comparación de métodos para acelerar análisis dinámicos de grandes estructuras con parametrizaciones, se han descrito ciertos tipos de fenómenos dinámicos específicos como son la supervisión de la integridad estructural, que trata de detectar daño estructural y prever el comportamiento de la estructura tras ese daño, la variación del comportamiento de la estructura debida a una delaminación o un crecimiento de grieta, la pérdida de pala sufrida por el motor de un avión en vuelo o modificaciones para alterar la respuesta dinámica de construcciones civiles como puentes o edificios frente a cargas sísmicas. Todos estos fenómenos pueden entenderse como modificaciones estructurales que varían la respuesta estructural bajo cargas dinámicas debido a los cambios que se producen en las propiedades físicas de la estructura.

A continuación se han introducido y clasificado brevemente los distintos tipos de métodos de reducción de modelos de elementos finitos que se recogen en la literatura.

Posteriormente, en el capítulo 2, se describe la base teórica del método propuesto recientemente de aceleración de análisis para entender su alcance y características en detalle, y que se denomina en esta tesis Método DACMAM. De forma análoga, en el capítulo que le sigue, se describen las ecuaciones en las que se basan el resto de métodos de la comparación. En el capítulo cuarto se recogen las primeras consideraciones que comparan los métodos tras su exposición teórica.

El sexto capítulo aborda la aplicación práctica y estudio de los resultados obtenidos de los diversos métodos en un caso de estudio basado en el análisis de una estructura de un edificio de 40 plantas sometido a cargas sísmicas, cuyos detalles está descritos en el capítulo 5. Se muestran los resultados considerando diferentes parametrizaciones de rigidez, masa y amortiguamiento.

La discusión y conclusiones se recogen en el capítulo final, donde también se analiza el cumplimiento de los objetivos de la Tesis.

Por último, se proponen ciertas líneas de investigación relacionadas con el objeto del presente trabajo para desarrollar en el futuro. 


\section{Capítulo 2}

\section{MÉTODO DE ACELERACIÓN DE}

ANÁLISIS DINÁMICOS EN EL

\section{ESPACIO MODAL COMPLEJO}

\section{$(D A C M A M)$}

Se muestra a continuación la descripción del nuevo método. Por motivos de fidelidad con el procedimiento se mantiene el esquema empleado en la presentación del método (Herrada-Martín [2016]).

El método que se describe emplea la superposición modal en el espacio complejo y tiene como característica principal que sólo emplea parte de la información de los modos, aquella relevante para los puntos donde se estudia la respuesta y donde se introduce la carga. El método muestra su mayor potencial cuando el número de estos puntos es reducido en modelos grandes, lo cual es bastante habitual. Adicionalmente permite modificaciones correspondientes a masas, rigideces y amortiguamientos en nodos del modelo existentes o en localizaciones nuevas mediante la creación de grados de libertad adicionales. De este modo el método es especialmente adecuado para el caso de parametrizaciones con este tipo de modificaciones.

El método no es exacto en el caso de truncatura modal (inevitable en modelos grandes), pero la aproximación que proporciona es suficiente si los cambios en la estructura no son importantes 
en términos de masa global, rigidez o amortiguamiento. Si no se emplea truncatura modal, el método es exacto.

En esta tesis se le denominará método para aceleración de análisis dinámicos en el espacio modal complejo (Dynamic Analysis in Complex Modal space Acceleration Method, DACMAM).

\subsection{Descomposición modal}

Se parte de la ecuación dinámica habitual,

$$
\mathbf{M} \ddot{\mathbf{x}}+\mathbf{C} \dot{\mathbf{x}}+\mathbf{K x}=\mathbf{P} f(t)
$$

donde $\mathbf{x}$ es el vector de desplazamiento de los nodos, de tamaño $G$ (número total de los grados de libertad), $\mathbf{M}$ es la matriz de masas, $\mathbf{C}$ es la matriz de amortiguamiento, $\mathbf{K}$ es la matriz de rigidez, $\mathbf{P}$ es el vector de cargas (vector de influencia) y $f(t)$, en función del tiempo, es el multiplicador de la carga (se considera por sencillez comportamiento proporcional para todos los grados de libertad, la ampliación del método a funciones distintas para cada grado de libertad es trivial). Se denominará $\mathbf{X}$ al vector de coordenadas (de longitud igual al número de nodos por el número de grados de libertad por nodo).

Considerando la superposición modal de los desplazamientos habitual:

$$
\mathbf{x}(\mathbf{X}, t)=\mathbf{\Phi}(\mathbf{X}) \mathbf{u}(t) \Leftrightarrow \mathbf{x}(\mathbf{X}, t)=\sum_{\substack{k=1 \\ i=c(k)}}^{n} u_{c(k)}(t) \phi_{c(k)}(\mathbf{X})+\sum_{\substack{k=1 \\ i=n c(k)}}^{G-n} u_{n c(k)}(t) \phi_{n c(k)}(\mathbf{X})
$$

donde $u_{i}(t)$ son los desplazamientos o coordenadas modales y $\mathbf{\Phi}(\mathbf{X})$ es la matriz de modos, cuyas columnas, $\boldsymbol{\phi}_{i}(\mathbf{X})$, son los modos de vibración de la estructura (reales si se cumplen ciertas condiciones), con $i=1, \ldots, G$. Esta matriz se ha particionado, siendo $n \leq G$ el número de modos que se consideran afectados por las modificaciones (grupo Considerado), en general un número mucho menor que el número de modos real de la estructura (e.g. cientos frente cientos de miles), i.e.

$$
\underbrace{\Phi_{c}}_{G \times n}=\left[\phi_{c(1)}, \phi_{c(2)}, \phi_{c(3)}, \ldots, \phi_{c(n)}\right] \quad \text { y } \quad \phi_{i}=\left[\phi_{1 i}, \ldots, \phi_{G i}\right]^{T}
$$


y $G-n$ es el número de modos que no se considera afectado por las modificaciones. Hasta ahora no se ha impuesto ningún tipo de limitación, pudiendo ser la cantidad de elementos de dichos grupos cualquiera al igual que el orden. La contribución a la respuesta de este segundo grupo se añadirá como una suma al resultado final.

$$
\underbrace{\Phi_{n c}}_{G \times(G-n)}=\left[\phi_{n c(1)}, \phi_{n c(2)}, \phi_{n c(3)}, \ldots, \phi_{n c(G-n)}\right]
$$

Si no hay amortiguamiento en la estructura (o este es proporcional), los modos son reales y la solución del problema de autovalores de la forma

$$
\left[\mathbf{K}-\omega_{i}^{2} \mathbf{M}\right] \phi_{i}=\mathbf{0}
$$

donde $\tilde{\mathbf{\Omega}}^{2}=\operatorname{diag}\left(\omega_{i}^{2}\right)$ es la matriz de autovalores reales sin amortiguamiento. Por sencillez de notación, durante el desarrollo teórico, se considerará la matriz modal completa $\tilde{\boldsymbol{\Phi}}$ y la matriz de autovalores completa $\tilde{\Omega}^{2}$ (ambas de tamaño $G \times G$ ), mientras que para referirse al grupo Considerado (de tamaño $G \times n$ o $n \times 1$ ), se omitirán los subíndices hasta el resultado final. Como es bien sabido, al ser todos los modos independientes se puede introducir la normalización de modos usual con la masa $\tilde{\mathbf{\Phi}}^{T} \mathbf{M} \tilde{\mathbf{\Phi}}=\mathbf{I}$ de modo que la ecuación de movimiento queda como sigue

$$
\ddot{\mathbf{u}}+\tilde{\boldsymbol{\Phi}}^{T} \mathbf{C} \tilde{\mathbf{\Phi}} \dot{\mathbf{u}}+\tilde{\mathbf{\Omega}}^{2} \mathbf{u}=\tilde{\mathbf{\Phi}}^{T} \mathbf{P} f(t)
$$

Las condiciones para que los autovectores del sistema sean reales son que $\mathbf{C}$ se pueda escribir como función lineal de las matrices K, M y sus inversas (Rayleigh [1945]; Caughey [1960]; Caughey y O'Kelly [1965]). También, bajo las condiciones habituales de bajo amortiguamiento en las estructuras, se puede considerar que la matriz de amortiguamiento modal, $\tilde{\boldsymbol{\Phi}}^{T} \mathbf{C} \tilde{\boldsymbol{\Phi}}$, es diagonal

$$
\tilde{\boldsymbol{\Phi}}^{T} \mathbf{C} \tilde{\boldsymbol{\Phi}}=\operatorname{diag}\left(2 \zeta_{i} \omega_{i}\right), \quad i=1, . ., G
$$

donde $\zeta_{i}$ es el "factor de amortiguamiento modal", correspondiente al del sistema de un grado de libertad del modo $i$ en el problema desacoplado resultante por ser las matrices del sistema, $\tilde{\mathbf{\Omega}}^{2}, \quad \tilde{\boldsymbol{\Phi}}^{T} \mathbf{C} \tilde{\boldsymbol{\Phi}}$ y $\quad \tilde{\boldsymbol{\Phi}}^{T} \mathbf{M} \tilde{\boldsymbol{\Phi}}=\mathbf{I}$, diagonales con el número de modos seleccionado de manera 
arbitraria. Si se denomina $\quad \overline{\mathbf{C}}=\boldsymbol{\Phi}^{T} \mathbf{C \Phi}$ a la matriz modal de amortiguamiento con la partición de $n$ modos indicada antes, la ecuación de movimiento en términos de las coordenadas modales correspondientes es

$$
\ddot{\mathbf{u}}+\overline{\mathbf{C}} \dot{\mathbf{u}}+\boldsymbol{\Omega}^{2} \mathbf{u}=\boldsymbol{\Phi}^{T} \mathbf{P} f(t)
$$

Cabe resaltar que para conocer la respuesta de un grado de libertad determinado, únicamente es necesario considerar las componentes de los modos en ese grado de libertad. Por esto, en la práctica, se puede reducir adicionalmente el problema de superposición modal únicamente a aquellos $g<<G$ grados de libertad de interés y considerar $\boldsymbol{\Phi}$ como una matriz de dimensión $g \times n$.

\subsection{Introducción de amortiguamiento adicional no proporcional. Nuevo problema de autovalores}

El hecho de emplear amortiguamiento proporcional en la estructura sin modificar es una hipótesis no real pero es bien conocido que es una aproximación aceptable en la mayoría de los casos si el amortiguamiento es pequeño (Schwarz y Richardson [2013]; Montáns y Muñoz [2013]). Tiene ventajas en eficiencia muy relevantes debido a que da lugar a ecuaciones en el espacio modal de autovalores reales que están desacopladas. Adicionalmente, determinar la matriz de amortiguamiento real en grandes estructuras es una tarea con un alto nivel de dificultad.

Hay casos en los que la estructura tiene dispositivos amortiguadores en localizaciones específicas como sistemas masa-amortiguador sintonizados para aliviar la respuesta dinámica a una o varias frecuencias determinadas. Aunque se pueden emplear aproximaciones para seguir tratando el problema en el espacio modal real (Bilbao et al. [2006]), en general se requiere la solución exacta, ya que el amortiguamiento real no puede aproximarse por uno proporcional, y la matriz de amortiguamiento modal ya no es diagonal. Aun así, el número de términos fuera de la diagonal no nulos es reducido, por lo que todavía se pueden realizar estudios paramétricos y diversos tipos de análisis con un tamaño del problema muy reducido, teniendo en cuenta únicamente los grados de libertad de los modos que se consideran afectados por la modificación (la partición que se comentaba inicialmente de los modos) y manteniendo el amortiguamiento modal proporcional en el resto de la estructura. Si se considera la siguiente partición de la 
matriz de amortiguamiento

$$
\mathbf{C}^{*}=\mathbf{C}+\mathbf{C}^{a}
$$

donde $\mathbf{C}$ es tal que $\quad \overline{\mathbf{C}}=\boldsymbol{\Phi}^{T} \mathbf{C} \boldsymbol{\Phi}=\operatorname{diag}\left(2 \zeta_{i} \omega_{i}\right)$ es la matriz de amortiguamiento considerada anteriormente, habitualmente con un amortiguamiento pequeño, y $\mathbf{C}^{a}$ es la matriz de amortiguamiento no proporcional, que debido a los mencionados dispositivos de amortiguamiento, no es necesariamente pequeña. Proyectando al subespacio modal se tiene

$$
\overline{\mathbf{C}}^{*}=\boldsymbol{\Phi}^{T} \mathbf{C}^{*} \boldsymbol{\Phi}=\overline{\mathbf{C}}+\boldsymbol{\Phi}^{T} \mathbf{C}^{a} \boldsymbol{\Phi}:=\overline{\mathbf{C}}+\overline{\mathbf{C}}^{a}
$$

Consideremos ahora $N$ amortiguadores entre los grados de libertad del grupo seleccionado $g$ con los siguientes valores de amortiguamiento: $\mathbf{c}=\left[c_{1}, c_{2}, \ldots, c_{N}\right]^{T}$. La matriz $\mathbf{C}^{a}$ está poblada con muchos ceros porque únicamente son no nulos los grados de libertad afectados por los elementos de c. Por ejemplo, en el caso de un único amortiguador que une 2 grados de libertad con una constante $c$, la matriz quedaría del siguiente modo

$$
\mathbf{C}^{a}=\left[\begin{array}{cccccccc}
\ddots & & & & & & \\
& 0 & 0 & 0 & 0 & 0 & \\
& 0 & c & 0 & -c & 0 & \\
& 0 & 0 & 0 & 0 & 0 & \\
0 & -c & 0 & c & 0 & \\
0 & 0 & 0 & 0 & 0 & \\
& & & & & \ddots
\end{array}\right]
$$

Por ello $\quad \overline{\mathbf{C}}^{a}=\boldsymbol{\Phi}^{T} \mathbf{C}^{a} \boldsymbol{\Phi}$ se puede obtener de la expresión

$$
\begin{aligned}
\bar{C}_{i j}^{a} & =\sum_{p=1}^{g} \sum_{q=1}^{g} \phi_{p i} C_{p q}^{a} \phi_{q j} \\
& =\sum_{\substack{k=1 \\
r=\operatorname{dof}_{1}(k), s=\operatorname{dof}_{2}(k)}}^{N}\left(\phi_{r i} C_{r r}^{a} \phi_{r j}+\phi_{r i} C_{r s}^{a} \phi_{s j}+\phi_{s i} C_{s r}^{a} \phi_{r j}+\phi_{s i} C_{s s}^{a} \phi_{s j}\right)
\end{aligned}
$$


donde sólo las coordenadas modales de los grados de libertad afectados de los modos considerados deben ser tenidas en cuenta. La ecuación de movimiento proyectada en el espacio modal quedaría ahora

$$
\ddot{\mathbf{u}}+\overline{\mathbf{C}}^{*} \dot{\mathbf{u}}+\boldsymbol{\Omega}^{2} \mathbf{u}=\boldsymbol{\Phi}^{T} \mathbf{P} f(t)
$$

Ahora $\overline{\mathbf{C}}^{*}$ no es diagonal, pero la dimensión de la matriz es mucho más pequeña que la del problema original en el espacio físico $(n<<G)$. Para desacoplar las ecuaciones diferenciales el problema se puede reescribir en el espacio de los estados en términos de desplazamientos y velocidades modales:

$$
\mathbf{U}:=\left[\begin{array}{c}
\mathbf{u} \\
\mathbf{v}
\end{array}\right], \quad \dot{\mathbf{U}}:=\left[\begin{array}{c}
\dot{\mathbf{u}} \\
\dot{\mathbf{v}}
\end{array}\right]
$$

donde la expresión de ü se obtiene a partir de la Ec. (2.12)

$$
\ddot{\mathbf{u}}=: \dot{\mathbf{v}}=-\overline{\mathbf{C}}^{*} \dot{\mathbf{u}}-\boldsymbol{\Omega}^{2} \mathbf{u}+\boldsymbol{\Phi}^{T} \mathbf{P} f(t)
$$

La Ec. (2.12) quedaría del siguiente modo:

$$
\left[\begin{array}{c}
\dot{\mathbf{u}} \\
\dot{\mathbf{v}}
\end{array}\right]=\underbrace{\left[\begin{array}{cc}
\mathbf{0} & \mathbf{I} \\
-\boldsymbol{\Omega}^{2} & -\overline{\mathbf{C}}^{*}
\end{array}\right]}\left[\begin{array}{l}
\mathbf{u} \\
\mathbf{v}
\end{array}\right]+\underbrace{\left[\begin{array}{c}
\mathbf{0} \\
\boldsymbol{\Phi}^{T} \mathbf{P}
\end{array}\right]} f(t)
$$

A

B

O

$$
\dot{\mathbf{U}}=\mathbf{A} \mathbf{U}+\mathbf{B} f(t)
$$

Esta disposición del espacio de los estados, no simétrica, permite el uso de matrices de amortiguamiento no simétricas. Esto último implica una pequeña pérdida de eficiencia del método, que se puede optimizar en el caso de que todas las matrices originales del sistema dinámico fueran simétricas empleando un espacio de los estados simétricos como se indica más adelante en la tesis. 


\subsection{Adición de masas locales y elementos con rigidez.}

Añadir masas y muelles al sistema se hace de modo análogo al descrito para los amortiguadores. Para el caso de adición de rigideces se considera, del mismo modo, una partición de la matriz de rigidez en un término sin modificaciones $\mathbf{K}$ y un término de rigideces añadidas $\mathbf{K}^{a}$

$$
\mathbf{K}^{*}=\mathbf{K}+\mathbf{K}^{a}
$$

La proyección en el subespacio de los modos considerados inicialmente queda

$$
\overline{\mathbf{K}}^{*}=\boldsymbol{\Phi}^{T} \mathbf{K}^{*} \boldsymbol{\Phi}=\boldsymbol{\Omega}^{2}+\boldsymbol{\Phi}^{T} \mathbf{K}^{a} \boldsymbol{\Phi}:=\boldsymbol{\Omega}^{2}+\overline{\mathbf{K}}^{a}
$$

donde $\overline{\mathbf{K}}^{a}$ se obtendría de modo similar al caso de amortiguamiento, i.e. haciendo uso únicamente de los grados de libertad afectados por las rigideces añadidas y de los modos relevantes. La nueva matriz A del sistema en el espacio de los estados, Ec. (2.16), es

$$
\mathbf{A}=\left[\begin{array}{cc}
\mathbf{0} & \mathbf{I} \\
-\boldsymbol{\Omega}^{2}-\overline{\mathbf{K}}^{a} & -\overline{\mathbf{C}}^{*}
\end{array}\right]
$$

Del mismo modo se obtiene la matriz de masas mientras que la matriz $\mathbf{B}$ no se modifica. Se introducen masas adicionales a través de la matriz de masas adicional $\mathbf{M}^{a}$. Entonces

$$
\mathbf{M}^{*}=\mathbf{M}+\mathbf{M}^{a}
$$

y la proyección en el espacio modal reducido a los modos considerados queda

$$
\overline{\mathbf{M}}^{*}=\boldsymbol{\Phi}^{T} \mathbf{M}^{*} \boldsymbol{\Phi}=\mathbf{I}+\boldsymbol{\Phi}^{T} \mathbf{M}^{a} \boldsymbol{\Phi}:=\mathbf{I}+\overline{\mathbf{M}}^{a}
$$

donde $\overline{\mathbf{M}}^{a}$ se obtiene de modo análogo a $\overline{\mathbf{K}}^{a}$ y a $\overline{\mathbf{C}}^{a}$. La matriz final $\mathbf{A}$ es

$$
\mathbf{A}=\left[\begin{array}{cc}
\mathbf{0} & \mathbf{I}+\overline{\mathbf{M}}^{a} \\
-\mathbf{\Omega}^{2}-\overline{\mathbf{K}}^{a} & -\overline{\mathbf{C}}^{*}
\end{array}\right]
$$




\subsection{Grados de libertad adicionales}

La adición de masas, rigideces o amortiguadores puede hacer necesario también añadir un pequeño número $d$ de grados de libertad que no estuviesen considerados en el modelo original. En este caso, se deben ampliar los modos originales con los grados de libertad adicionales, de modo que se considere su influencia en la estructura original. Adicionalmente, se deben añadir tantos vectores a la matriz de modos como grados de libertad considerados, $d$ (por ejemplo para incluir nuevos modos locales). Se eligen estos modos teniendo valor unitario en los nuevos grados de libertad y el resto de términos nulos (de modo similar a vectores de inicio en el método de iteración de subespacios), que además de ser sencillo los hace ortogonales a los modos originales. De este modo se expande la matriz de modos

$$
\hat{\boldsymbol{\Phi}}:=\left[\begin{array}{cc}
\boldsymbol{\Phi} & \mathbf{0}_{n \times d} \\
\mathbf{0}_{n \times d}^{T} & \mathbf{1}_{d \times d}
\end{array}\right]
$$

La matriz de rigidez modal expandida sin modificaciones es

$$
\hat{\boldsymbol{\Phi}}^{T} \hat{\mathbf{K}} \hat{\boldsymbol{\Phi}}=\left[\begin{array}{ll}
\boldsymbol{\Omega}^{2} & \mathbf{0} \\
\mathbf{0}^{T} & \mathbf{0}
\end{array}\right]=: \hat{\boldsymbol{\Omega}}^{2}
$$

y, de modo similar, se expanden las matrices modales de masas y amortiguamiento

$$
\begin{gathered}
\hat{\boldsymbol{\Phi}}^{T} \hat{\mathbf{M}} \hat{\boldsymbol{\Phi}}=\left[\begin{array}{cc}
\boldsymbol{\Phi}^{T} \mathbf{M} \boldsymbol{\Phi} & \mathbf{0} \\
\mathbf{0}^{T} & \mathbf{0}
\end{array}\right]=\left[\begin{array}{cc}
\mathbf{I} & \mathbf{0} \\
\mathbf{0}^{T} & \mathbf{0}
\end{array}\right]=: \hat{\mathbf{I}} \\
\hat{\boldsymbol{\Phi}}^{T} \hat{\mathbf{C}} \hat{\boldsymbol{\Phi}}=\left[\begin{array}{cc}
\overline{\mathbf{C}} & \mathbf{0} \\
\mathbf{0}^{T} & 0
\end{array}\right]=: \hat{\overline{\mathbf{C}}}
\end{gathered}
$$

Aquí se debe tener en cuenta que las masas, rigideces o amortiguadores se pueden añadir en los nuevos grados de libertad de modo similar al indicado para los grados de libertad originales.

Los términos de masa, rigidez o amortiguamiento se incluyen igual que antes, pero aplicados sobre las matrices expandidas con los nuevos grados de libertad y autovectores, por ejemplo, 
para rigideces se tendría:

$$
\widehat{\bar{K}}_{i j}^{a}=\sum_{p=1}^{g+d} \sum_{q=1}^{g+d} \hat{\phi}_{p i} K_{p q}^{a} \hat{\phi}_{q j}
$$

donde $\hat{\phi}_{p i}=0$ para $p>n$ excepto $\hat{\phi}_{p p}=1$. Finalmente, la matriz $\hat{\mathbf{A}}$ expandida es

$$
\hat{\mathbf{A}}=\left[\begin{array}{cc}
\hat{\mathbf{0}} & \hat{\mathbf{I}}+\widehat{\overline{\mathbf{M}}}^{a} \\
-\hat{\mathbf{\Omega}}^{2}-\widehat{\mathbf{\mathbf { K }}}^{a} & -\widehat{\widehat{\mathbf{C}}}^{*}
\end{array}\right]
$$

En las secciones siguientes se omitirá el sombrero para denotar a las matrices expandidas sin pérdida de generalidad, puesto que no hay diferencia en el procedimiento una vez que se han expandido las matrices.

\subsection{Solución del problema general}

Con las matrices expandidas y modificadas, se consideran para la Ec. 2.16) soluciones del tipo

$$
\mathbf{U}(\mathbf{X}, t)=\boldsymbol{\Psi}(\mathbf{X}) \boldsymbol{\xi}(t)
$$

donde $\boldsymbol{\Psi}$ es la matriz de cambio de coordenadas, que convierte los modos reales del sistema original a modos complejos del modelo modificado (figura 2-1). Al mismo tiempo, son los autovectores complejos del nuevo problema de autovalores

$$
\mathbf{A} \boldsymbol{\Psi}=\mathbf{\Psi} \boldsymbol{\Lambda} \quad \text { con } \quad \boldsymbol{\Lambda}=\operatorname{diag}\left(\lambda_{i}\right)
$$

El vector $\boldsymbol{\xi}$ contiene los multiplicadores modales y $\lambda_{i}$ son los $2 n$ autovalores complejos del nuevo problema. Es sabido que los modos complejos y los autovalores complejos correspondientes de este tipo de problemas se presentan en pares conjugados. La Ec. (2.16) se escribe ahora de la forma

$$
\mathbf{\Psi} \dot{\boldsymbol{\xi}}=\mathbf{A} \boldsymbol{\Psi} \boldsymbol{\xi}+\mathbf{B} f(t)
$$

Ya que A es no simétrica (se recuerda que de este modo el procedimiento admite matrices 


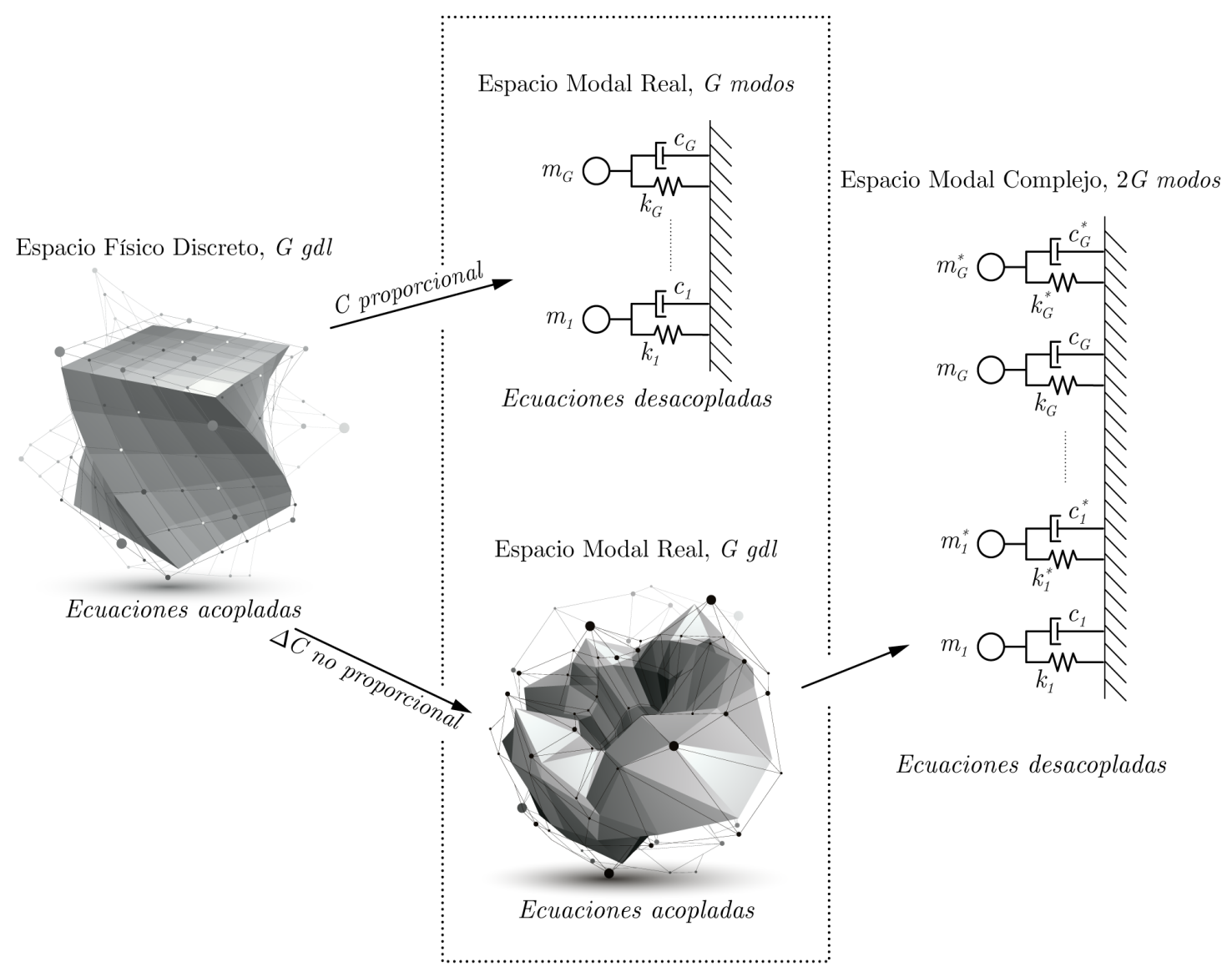

Figura 2-1: Esquema del método DACMAM: espacio físico, espacio modal real y espacio modal complejo.

de amortiguamiento y rigideces no simétricas), debe tenerse en consideración una nueva matriz de vectores $\boldsymbol{\Gamma}^{T}$, que se introducen premultiplicando la ecuación

$$
\boldsymbol{\Gamma}^{T} \boldsymbol{\Psi} \dot{\boldsymbol{\xi}}-\boldsymbol{\Gamma}^{T} \mathbf{A} \boldsymbol{\Psi} \boldsymbol{\xi}=\boldsymbol{\Gamma}^{T} \mathbf{B} f(t)
$$

de modo que $\gamma_{i}^{T} \mathbf{A} \psi_{j}=\lambda_{j} \gamma_{i}^{T} \boldsymbol{\psi}_{j}$, donde $\boldsymbol{\gamma}_{i}$ son las columnas de $\boldsymbol{\Gamma}$ (los autovectores de $\mathbf{A}^{T}$ ) y $\boldsymbol{\psi}_{i}$ las columnas de $\boldsymbol{\Psi}$ :

$$
\boldsymbol{\Gamma}^{T} \mathbf{A} \Psi=\Lambda \boldsymbol{\Gamma}^{T} \boldsymbol{\Psi}
$$

Para simplificar la Ec. (2.31) se emplea la normalización habitual a partir de las condiciones 
de ortogonalidad del problema complejo. Por la definición de $\boldsymbol{\Psi}$ y $\boldsymbol{\Gamma}$ se tiene:

$$
\begin{aligned}
& \gamma_{i}^{T}\left(\mathbf{A} \psi_{j}\right)=\gamma_{i}^{T} \lambda_{j} \boldsymbol{\psi}_{j} \\
& \left(\boldsymbol{\gamma}_{i}^{T} \mathbf{A}\right) \boldsymbol{\psi}_{j}=\lambda_{i} \boldsymbol{\gamma}_{i}^{T} \boldsymbol{\psi}_{j}
\end{aligned}
$$

y

$$
0=\left(\lambda_{j}-\lambda_{i}\right) \boldsymbol{\gamma}_{i}^{T} \boldsymbol{\psi}_{j} \Rightarrow \text { si } \lambda_{i} \neq \lambda_{j} \text { entonces } \boldsymbol{\gamma}_{i}^{T} \boldsymbol{\psi}_{j}=0
$$

de modo que $\boldsymbol{\Gamma}^{T} \boldsymbol{\Psi}$ es una matriz diagonal $\left(\boldsymbol{\gamma}_{i}\right.$ y $\boldsymbol{\psi}_{j}$ son ortogonales). Se pueden redefinir los autovectores para obtener $\boldsymbol{\Gamma}^{T} \boldsymbol{\Psi}=\mathbf{I}$; por ejemplo

$$
\boldsymbol{\psi}_{i} \leftarrow \frac{1}{\left(\gamma_{i}^{T} \boldsymbol{\psi}_{i}\right)} \boldsymbol{\psi}_{i}
$$

y la Ec. (2.31) queda ahora de la forma

$$
\dot{\boldsymbol{\xi}}-\boldsymbol{\Lambda} \boldsymbol{\xi}=\boldsymbol{\Gamma}^{T} \mathbf{B} f(t)
$$

que es ahora un sistema desacoplado de $2 n$ ecuaciones diferenciales

$$
\dot{\xi}_{i}-\lambda_{i} \xi_{i}=b_{i} f(t) \quad \text { con } \quad b_{i}=\gamma_{i}^{T} \mathbf{B}
$$

cuya solución es

$$
\xi_{i}(t)=e^{-h}\left[\int_{0}^{t} e^{-\lambda_{i} \tau} b_{i} f(\tau) d \tau+\text { const }\right] \quad \text { con } \quad h=\int_{0}^{t}\left(-\lambda_{i}\right) d t=-\lambda_{i} t
$$

Para condiciones iniciales no nulas, $\xi_{i}(0)=\xi_{i 0} \neq 0$, la solución es como sigue:

$$
\begin{aligned}
\xi_{i}(t) & =b_{i} \int_{0}^{t} e^{\lambda_{i}(t-\tau)} f(\tau) d \tau+\xi_{i}^{0}(t) \\
& =b_{i} \int_{0}^{t} e^{\lambda_{i}(t-\tau)} f(\tau) d \tau+e^{\lambda_{i} t} \xi_{i 0}
\end{aligned}
$$

Si se considera a $f(t)$ como una superposición de $M$ funciones trigonométricas (como puede 
ser aplicando la transformada de Fourier a una excitación general)

$$
f(t)=\sum_{m=1}^{M} a_{m} \sin \left(\varpi_{m} t+\varphi_{m}\right)
$$

Entonces la solución se puede obtener por superposición a partir de

$$
\xi_{i}(t)=\sum_{m=1}^{M} \bar{\xi}_{i m}(t)+\xi_{i}^{0}(t)
$$

donde, para cada término $m$, la solución es

$$
\begin{aligned}
\bar{\xi}_{i m}(t)= & a_{m} b_{i} \int_{0}^{t} e^{(t-\tau) \lambda_{i}} \sin \left(\varpi_{m} \tau+\varphi_{m}\right) d \tau \\
= & \frac{a_{m} b_{i}}{\lambda_{i}^{2}+\varpi_{m}^{2}}\left[\varpi_{m}\left(\cos \varphi_{m}\right) e^{t \lambda_{i}}+\lambda_{i}\left(\sin \varphi_{m}\right) e^{t \lambda_{i}}\right. \\
& \left.-\varpi_{m} \cos \left(\varphi_{m}+t \varpi_{m}\right)-\lambda_{i} \sin \left(\varphi_{m}+t \varpi_{m}\right)\right]
\end{aligned}
$$

y

$$
\xi_{i}^{0}(t)=e^{\lambda_{i} t} \xi_{i 0} \quad \text { con } \quad \boldsymbol{\xi}_{0}=\boldsymbol{\Psi}^{-1} \mathbf{U}(\mathbf{X}, 0)
$$

En general, el interés reside en la parte permanente de la respuesta. En tal caso, se puede descomponer el autovalor complejo en sus partes real e imaginaria

$$
\lambda_{i}=\sigma_{i}+j \omega_{d i}
$$

de tal modo que

$$
e^{\lambda_{i} t}=e^{\sigma_{i} t} e^{j \omega_{d i} t}
$$

y

$$
\begin{aligned}
\bar{\xi}_{i m}(t)= & \frac{a_{m} b_{i}}{\lambda_{i}^{2}+\varpi_{m}^{2}}[\underbrace{-\varpi_{m} \cos \left(\varphi_{m}+t \varpi_{m}\right)-\lambda_{i} \sin \left(\varphi_{m}+t \varpi_{m}\right)}_{\text {permanente }} \\
& \underbrace{\left.+\varpi_{m}\left(\cos \varphi_{m}\right) e^{\sigma_{i} t} e^{j \omega_{d i} t}+\lambda_{i}\left(\sin \varphi_{m}\right) e^{\sigma_{i} t} e^{j \omega_{d i} t}\right]}
\end{aligned}
$$

transitoria 
Para respuestas acotadas con $\sigma<0$, se tiene para la respuesta permanente

$$
\begin{aligned}
\bar{\xi}_{i m}^{\infty}:=\bar{\xi}_{i m}(t \rightarrow \infty) & = \\
& =\frac{-a_{m} b_{i}}{\lambda_{i}^{2}+\varpi_{m}^{2}}\left[\varpi_{m} \cos \left(t \varpi_{m}+\varphi_{m}\right)+\lambda_{i} \sin \left(t \varpi_{m}+\varphi_{m}\right)\right] \\
& =\bar{A}_{i m} \sin \left(t \varpi_{m}+\varphi_{m}\right)+\bar{B}_{i m} \cos \left(t \varpi_{m}+\varphi_{m}\right)
\end{aligned}
$$

y la respuesta transitoria es $\xi_{i}^{t}:=\xi_{i}^{0}+\sum_{m=1}^{M} \bar{\xi}_{i m}^{t}$, donde $\quad \xi_{i}^{0}=e^{\lambda_{i} t} \xi_{i 0} \quad$ y $\bar{\xi}_{i m}^{t}$ es

$$
\bar{\xi}_{i m}^{t}:=-e^{\sigma_{i} t} e^{j \omega_{d i} t}\left[\bar{B}_{i m} \cos \varphi_{m}+\bar{A}_{i m} \sin \varphi_{m}\right]
$$

con las amplitudes

$$
\bar{A}_{i m}=\frac{-b_{i} \lambda_{i}}{\lambda_{i}^{2}+\varpi_{m}^{2}} a_{m} \quad \text { y } \quad \bar{B}_{i m}=\frac{-b_{i} \varpi_{m}}{\lambda_{i}^{2}+\varpi_{m}^{2}} a_{m}
$$

De este modo, las operaciones para la respuesta permanente se pueden obtener en función de estas amplitudes

$$
\xi_{i}^{\infty}(t)=\sum_{m=1}^{M} \bar{\xi}_{i m}^{\infty}(t) \Rightarrow \boldsymbol{\xi}^{\infty}=\sum_{m=1}^{M} \overline{\boldsymbol{\xi}}_{m}^{\infty}=\sum_{m=1}^{M}\left[\overline{\mathbf{A}}_{m} \sin \left(t \varpi_{m}+\varphi_{m}\right)+\overline{\mathbf{B}}_{m} \cos \left(t \varpi_{m}+\varphi_{m}\right)\right]
$$

donde $\overline{\mathbf{A}}_{m}$ y $\overline{\mathbf{B}}_{m}$ son vectores, siendo $\bar{A}_{i m}$ el término correspondiente al modo $i$. De este modo, si se recupera la expresión de la Ec. (2.28) :

$$
\begin{gathered}
\mathbf{U}=\boldsymbol{\Psi}(\mathbf{X}) \boldsymbol{\xi}(\mathbf{t}) \\
\mathbf{U}^{\infty}=\left[\begin{array}{l}
\mathbf{u} \\
\mathbf{v}
\end{array}\right]^{\infty}=\sum_{m=1}^{M}\left(\boldsymbol{\Psi} \overline{\mathbf{A}}_{m} \sin \left(t \varpi_{m}+\varphi_{m}\right)+\boldsymbol{\Psi} \overline{\mathbf{B}}_{m} \cos \left(t \varpi_{m}+\varphi_{m}\right)\right)
\end{gathered}
$$

y a partir de la Ec. (2.13), se puede realizar la siguiente partición:

$$
\boldsymbol{\Psi} \overline{\mathbf{A}}_{m}=\left[\begin{array}{c}
\left(\boldsymbol{\Psi} \overline{\mathbf{A}}_{m}\right)^{U} \\
\left(\boldsymbol{\Psi} \overline{\mathbf{A}}_{m}\right)^{L}
\end{array}\right] ; \quad \boldsymbol{\Psi} \overline{\mathbf{B}}_{m}=\left[\begin{array}{c}
\left(\boldsymbol{\Psi} \overline{\mathbf{B}}_{m}\right)^{U} \\
\left(\boldsymbol{\Psi} \overline{\mathbf{B}}_{m}\right)^{L}
\end{array}\right]
$$


Entonces

$$
\mathbf{x}^{\infty}=\mathbf{\Phi} \mathbf{u}^{\infty}=\sum_{m=1}^{M}[\underbrace{\boldsymbol{\Phi}\left(\boldsymbol{\Psi} \overline{\mathbf{A}}_{m}\right)^{U}}_{:=\hat{\mathbf{a}}_{m}^{\infty}} \sin \left(t \varpi_{m}+\varphi_{m}\right)+\underbrace{\mathbf{\Phi}\left(\boldsymbol{\Psi} \overline{\mathbf{B}}_{m}\right)^{U}}_{:=\hat{\mathbf{b}}_{m}^{\infty}} \cos \left(t \varpi_{m}+\varphi_{m}\right)]
$$

Adicionalmente, la parte transitoria de la respuesta se puede calcular a partir de las ecuaciones $(2.49)$ y $(2.50)$ :

$$
\begin{aligned}
\mathbf{x}^{t}=\boldsymbol{\Phi} \mathbf{u}^{t}= & -\sum_{m=1}^{M}[\underbrace{\boldsymbol{\Phi}\left(\boldsymbol{\Psi} \mathbf{e}^{\boldsymbol{\Lambda t}} \overline{\mathbf{A}}_{m}\right)^{U} \sin \varphi_{m}}_{:=\hat{\mathbf{a}}_{m}^{t}(t)}+\underbrace{\boldsymbol{\Phi}\left(\boldsymbol{\Psi} \mathbf{e}^{\boldsymbol{\Lambda} \mathbf{t}} \overline{\mathbf{B}}_{m}\right)^{U} \cos \varphi_{m}}_{:=\hat{\mathbf{b}}_{m}^{t}(t)}] \\
& +\underbrace{\boldsymbol{\Phi}\left(\boldsymbol{\Psi} \mathbf{e}^{\boldsymbol{\Lambda} \mathbf{t}} \boldsymbol{\Psi}^{-1}\left[\begin{array}{c}
\mathbf{u}_{0} \\
\mathbf{v}_{0}
\end{array}\right]\right)}_{:=\hat{\mathbf{c}}^{t}(t)}
\end{aligned}
$$

Finalmente, para el caso más general, añadiendo la contribución de los modos no considerados para las modificaciones, se obtiene:

$$
\mathbf{x}(t)=\mathbf{x}^{t}(t)+\mathbf{x}^{\infty}(t)+\sum_{\substack{k=1 \\ i=n c(k)}}^{G-n} u_{n c(k)}(t) \phi_{n c(k)}(\mathbf{X})
$$

\subsection{Ejemplo de aplicación}

En la presentación del método (Herrada-Martín [2016]) se mostraba un caso práctico con una gran estructura que correspondía al ala de una aeronave no tripulada (2-2). A modo de ejemplo, para mostrar las capacidades del método, se muestran en la tabla (2.1) los resultados de tiempos de análisis de dicho caso práctico para una parametrización que resuelve un total de 115000 análisis. En dicha tabla, modelo reducido indica que se ha resuelto el método en el espacio modal con truncatura de modos, mientras que el modelo completo corresponde a una integración numérica con un algoritmo implícito.

También se mostraban datos acerca del volumen de ficheros generados, siendo del orden de cientos de MB para los resultados del código comercial, frente a KB para el método DACMAM. 


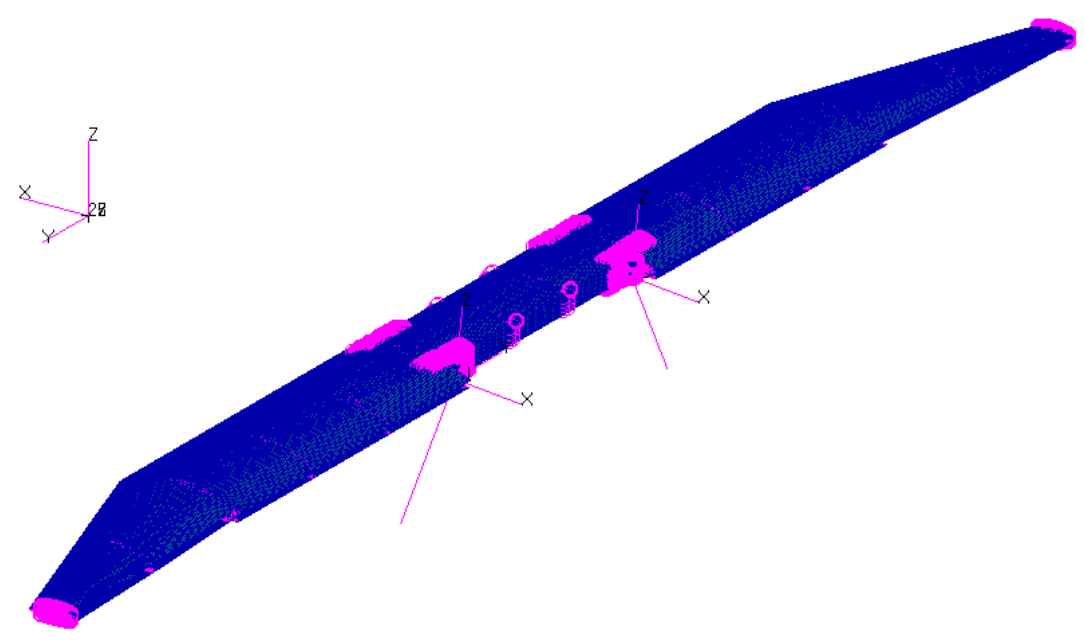

Figura 2-2: FEM completo del ala de una aeronave no tripulada (cortesía INTA).

\begin{tabular}{|c|c|c|}
\hline Tipo de análisis & Tiempo por análisis (s) & Tiempo total (h) \\
\hline Modelo completo con código comercial & 4500 & 96000 \\
\hline Modelo reducido con código comercial & 1200 & 25600 \\
\hline Modelo reducido con método DACMAM & 1.1 & 23.4 \\
\hline
\end{tabular}

Tabla 2.1: Tiempos de computación de los diferentes análisis y modelos del ala de una aeronave no tripulada. 


\section{Capítulo 3}

\section{OTROS MÉTODOS DE REDUCCIÓN DE MODELOS}

\subsection{Técnicas de reducción consideradas}

Para estudiar las ventajas y límites del nuevo método, se compara con otros métodos de reducción que son empleados actualmente en las empresas y organismos dedicados al análisis estructural.

De las técnicas o métodos descritos en la introducción para la reducción de modelos estructurales en el campo de la dinámica estructural y las vibraciones, se han escogido para la comparación los métodos de condensación dinámica que admiten un amortiguamiento no clásico o no proporcional, ya que es una de las capacidades del nuevo método, y se han descartado aquellos métodos que admiten sólo modelos estructurales sin amortiguamiento o amortiguamiento clásico. En Paz [1984], Paz [1989], Qu [2004], Besselink [2013] se pueden encontrar los métodos aquí seleccionados y otros que sólo admiten amortiguamiento nulo o amortiguamiento diagonalizable en el espacio modal real.

Se puede considerar que todos estos métodos de condensación derivan del método de condensación estática de Guyan [1965] o B.Irons [1965]. Los métodos seleccionados para la comparación se pueden agrupar asimismo en dos grupos: métodos de reducción iterativos y métodos de reducción directos.

Los primeros proporcionan mejores resultados, mientras que los segundos proporcionan tiem- 
pos de computación menores penalizando la calidad de los resultados.

Los métodos iterativos son una mejora de los métodos directos que permiten resolver de manera más exacta las ecuaciones que definen el problema, que son no lineales. Para un mismo método iterativo existen diversas variantes que sólo modifican la aproximación inicial de la solución, dando lugar a tiempos de computación y número de iteraciones necesario para alcanzar la convergencia distintos.

A continuación, tras un apartado en que se introduce la resolución de sistemas por medio de álgebra compleja, se describirán brevemente los métodos. Se empleará para referirse a los distintos métodos una nomenclatura que identifica el espacio de los estados que se emplea, similar a la empleada en Qu [2004]. En todos se parte de la ecuación de equilibrio dinámico con la presencia de amortiguamiento, de dimensión $G$ :

$$
\mathbf{M} \ddot{\mathbf{x}}+\mathbf{C} \dot{\mathbf{x}}+\mathbf{K x}=\mathbf{P} f(t)
$$




\section{2. Álgebra compleja}

Los métodos que se describen a continuación y el método $D A C M A M$ requieren del álgebra compleja para su resolución.

Para una descripción más detallada de la matemática implicada se puede revisar diversas referencias, habiendo referencias específicas de análisis de estructuras como Chopra [1995], Harris et al. [2002], o Qu [2004].

Partiendo de la Ec. (3.1), para cargas aplicadas nulas, se plantea el problema de autovalores considerando, como se hace habitualmente, una solución de la forma

$$
\mathbf{x}=\phi \mathbf{e}^{\lambda t}
$$

donde el vector $\phi$ y el coeficiente $\lambda$ son números complejos en el caso más general. El modo que se emplea habitualmente para resolver este problema con el fin de poder desacoplar las ecuaciones diferenciales, es transformarlo en un problema de segundo orden, similar al problema de autovalores clásico de sistemas no amortiguados, mediante la introducción de un vector que se denomina vector de estados (se introducen más adelante varios métodos para hacerlo que dan lugar a espacios vectoriales con bases distintas). La diferencia entre ambos sistemas radica en que la matriz del nuevo sistema que multiplica la derivada del vector de estados no es definida positiva, mientras que la matriz de masas del sistema original sí lo era. El vector columna introducido y su derivada, de dimensión $2 G$, tienen la forma:

$$
\mathbf{X}=\left[\begin{array}{c}
\mathbf{x} \\
\dot{\mathbf{x}}
\end{array}\right], \dot{\mathbf{X}}=\left[\begin{array}{c}
\dot{\mathrm{x}} \\
\ddot{\mathbf{x}}
\end{array}\right]
$$

El "vector de estado", X, y la "velocidad del vector de estado", $\dot{\mathbf{X}}$, se denominan de este modo porque contienen el desplazamiento y la velocidad de la estructura, que una vez conocidos, por la ecuación de equilibrio determina la aceleración, quedando la solución o estado del sistema unívocamente definido. El problema de autovalores queda

$$
\mathbf{A X}+\mathbf{B} \dot{\mathbf{X}}=\mathbf{F} \Rightarrow(\mathbf{A}+\lambda \mathbf{B}) \boldsymbol{\psi}=\mathbf{0}, \quad \text { siendo } \boldsymbol{\psi}=\left[\begin{array}{c}
\boldsymbol{\phi} \\
\lambda \boldsymbol{\phi}
\end{array}\right]
$$


La solución completa al problema de autovalores descrito serán $2 G$ autovectores, que son pares conjugados con sus correspondientes autovalores. En el caso de que las matrices del sistema no sean simétricas, se debe resolver adicionalmente el problema de autovalores compuesto de las matrices transpuestas (los autovalores son coincidentes).

$$
\boldsymbol{\gamma}^{T}(\mathbf{A}+\lambda \mathbf{B})=\mathbf{0} \Rightarrow\left(\mathbf{A}^{T}+\lambda \mathbf{B}^{T}\right) \boldsymbol{\gamma}=\mathbf{0}
$$

Se pueden ensamblar las matrices de autovectores con el siguiente orden

$$
\boldsymbol{\Psi}=\left[\boldsymbol{\psi}_{1}, \ldots, \boldsymbol{\psi}_{G}, \boldsymbol{\psi}_{1}^{*}, \ldots, \boldsymbol{\psi}_{G}^{*}\right], \quad \boldsymbol{\Gamma}=\left[\gamma_{1}, \ldots, \gamma_{G}, \boldsymbol{\gamma}_{1}^{*}, \ldots, \gamma_{G}^{*}\right]
$$

y la matriz diagonal de autovalores

$$
\boldsymbol{\Lambda}=\left[\begin{array}{cc}
\operatorname{diag}\left(\lambda_{1}, \ldots, \lambda_{G}\right) & \mathbf{0} \\
\mathbf{0} & \operatorname{diag}\left(\lambda_{1}^{*}, \ldots, \lambda_{G}^{*}\right)
\end{array}\right]
$$

A partir de ellas se describen las condiciones de ortogonalidad:

$$
\boldsymbol{\Gamma}^{T} \mathbf{A} \boldsymbol{\Psi}=-\boldsymbol{\Lambda} \quad y \quad \boldsymbol{\Gamma}^{T} \mathbf{B} \boldsymbol{\Psi}=\mathbf{I}
$$

Si $\mathbf{A}$ y $\mathbf{B}$ son simétricas se cumple la condición $(\mathbf{A}+\lambda \mathbf{B})=\left(\mathbf{A}^{T}+\lambda \mathbf{B}^{T}\right) \Rightarrow \boldsymbol{\Psi}=\boldsymbol{\Gamma}$.

De cada par conjugado de autovalores se pueden obtener las frecuencias propias y factor amortiguamiento crítico cuya interpretación en el espacio físico está clara. Tomando el par conjugado de autovalores del modo $r$ se tiene:

$$
\begin{aligned}
& \lambda_{r}=-\alpha_{r}+j \beta_{r} \quad, \quad \lambda_{r}^{*}=-\alpha_{r}-j \beta_{r} \Rightarrow \\
& \zeta_{r}=\alpha_{r} / \sqrt{\alpha_{r}^{2}+\beta_{r}^{2}}, \quad \omega_{r}=\sqrt{\alpha_{r}^{2}+\beta_{r}^{2}}, \quad \omega_{r}^{d}=\omega_{r} \sqrt{1-\zeta_{r}^{2}}
\end{aligned}
$$

donde $\omega_{r}$ se denomina frecuencia natural sin amortiguar del modo $r, \mathrm{y} \omega_{r}^{d}$ se denomina la frecuencia amortiguada del modo $r$.

Cabe resaltar que el tiempo de computación para resolver el nuevo problema de autovalores, que duplica la dimensión del primero puede ser hasta 8 veces mayor (el tiempo de computación 
de gran parte de los algoritmos de resolución de autovalores es proporcional al cubo de la dimensión de la diagonal). Hay muchos algoritmos para obtener los autovalores y autovectores de una matriz, dependiendo el óptimo del tamaño y otras características de las matrices involucradas, como son su simetría, si son definidas positivas o no, reales o complejas, tipo "sparse", etc. En dinámica estructural, debido a que se pueden encontrar diversos tipos de matrices, no se puede mencionar uno idóneo para todos los casos, pero cabe mencionar el método de Lanczos (Lanczos [1950]), con buenas propiedades para el gran tamaño que tienen habitualmente las matrices involucradas. Adicionalmente se debe tener en cuenta que en los problemas de autovalores de análisis de estructuras no es necesario obtener todos los autovalores y autovectores del sistema, siendo requeridos en general los correspondientes a un rango de frecuencias o un número determinado de los autovalores más cercanos a 0 . Se puede encontrar una revisión exhaustiva de muchos de los algoritmos disponibles en Golub y van der Vorst [2000]. 


\subsection{Condensación Estática}

\subsubsection{Condensación estática en el espacio de los desplazamientos (SCDS)}

La primera de las tres condensaciones consideradas es básicamente la condensación de Guyan para problemas dinámicos (Guyan [1965]), pero ampliada a un sistema con amortiguamiento. Dicha condensación se realiza en el espacio físico (a continuación, si se resuelve el sistema reducido en el espacio de los estados, permite emplear amortiguamiento no clásico, que da lugar a matrices de amortiguamiento modal no diagonales). Se emplea la notación habitual: "master" son los grados de libertad retenidos en la reducción, y "slave" son los grados de libertad que se condensan o eliminan mediante la siguiente transformación:

$$
\mathbf{x}=\left[\begin{array}{c}
\mathbf{x}_{m} \\
\mathbf{x}_{s}
\end{array}\right]=\left[\begin{array}{c}
\mathbf{I} \\
-\mathbf{K}_{s s}^{-1} \mathbf{K}_{s m}
\end{array}\right] \mathbf{x}_{m}=\mathbf{T} \mathbf{x}_{m}
$$

donde $\mathbf{T}$ se denomina matriz de transformación de coordenadas o matriz global de mapeado.

Con esta transformación, las ecuaciones de energía elástica y energía cinética quedan:

$$
\begin{aligned}
\mathcal{U} & =\frac{1}{2} \mathbf{x}^{T} \mathbf{K} \mathbf{x}=\frac{1}{2} \mathbf{x}_{m}^{T} \mathbf{T}^{T} \mathbf{K} \mathbf{T} \mathbf{x}_{m}=\frac{1}{2} \mathbf{x}_{m}^{T} \overline{\mathbf{K}} \mathbf{x}_{m} \\
\mathcal{K} & =\frac{1}{2} \dot{\mathbf{x}}^{T} \mathbf{M} \dot{\mathbf{x}}=\frac{1}{2} \dot{\mathbf{x}}_{m}^{T} \mathbf{T}^{T} \mathbf{M T} \dot{\mathbf{x}}_{m}=\frac{1}{2} \dot{\mathbf{x}}_{m}^{T} \overline{\mathbf{M}} \dot{\mathbf{x}}_{m}
\end{aligned}
$$

Por tanto, las matrices de masas y de rigideces reducidas, y el vector de cargas aplicadas resultan:

$$
\begin{aligned}
\overline{\mathbf{M}}=\mathbf{T}^{T} \mathbf{M} \mathbf{T}= & {\left[\begin{array}{ll}
\mathbf{I} & -\mathbf{K}_{m s} \mathbf{K}_{s s}^{-1}
\end{array}\right]\left[\begin{array}{cc}
\mathbf{M}_{m m} & \mathbf{M}_{m s} \\
\mathbf{M}_{s m} & \mathbf{M}_{s s}
\end{array}\right]\left[\begin{array}{c}
\mathbf{I} \\
-\mathbf{K}_{s s}^{-1} \mathbf{K}_{s m}
\end{array}\right]=} \\
& \mathbf{M}_{m m}-\mathbf{K}_{m s} \mathbf{K}_{s s}^{-1} \mathbf{M}_{s m}-\mathbf{M}_{m s} \mathbf{K}_{s s}^{-1} \mathbf{K}_{s m}+\mathbf{K}_{m s} \mathbf{K}_{s s}^{-1} \mathbf{M}_{s s} \mathbf{K}_{s s}^{-1} \mathbf{K}_{s m}
\end{aligned}
$$




$$
\begin{gathered}
\overline{\mathbf{K}}=\mathbf{T}^{T} \mathbf{K} \mathbf{T}=\left[\begin{array}{ll}
\mathbf{I} & -\mathbf{K}_{m s} \mathbf{K}_{s s}^{-1}
\end{array}\right]\left[\begin{array}{cc}
\mathbf{K}_{m m} & \mathbf{K}_{m s} \\
\mathbf{K}_{s m} & \mathbf{K}_{s s}
\end{array}\right]\left[\begin{array}{c}
\mathbf{I} \\
-\mathbf{K}_{s s}^{-1} \mathbf{K}_{s m}
\end{array}\right]= \\
\mathbf{K}_{m m}-\mathbf{K}_{m s} \mathbf{K}_{s s}^{-1} \mathbf{K}_{s m} \\
\overline{\mathbf{P}}=\mathbf{T}^{T} \mathbf{P}=\left[\begin{array}{ll}
\mathbf{I} & -\mathbf{K}_{m s} \mathbf{K}_{s s}^{-1}
\end{array}\right]\left[\begin{array}{c}
\mathbf{P}_{m} \\
\mathbf{P}_{s}
\end{array}\right]=\mathbf{P}_{m}-\mathbf{K}_{m s} \mathbf{K}_{s s}^{-1} \mathbf{P}_{s}
\end{gathered}
$$

Y de modo similar, la ecuación del trabajo debido a las fuerzas de la amortiguación y los desplazamientos virtuales, y la matriz de amortiguamiento reducida son:

$$
\begin{gathered}
\delta \mathcal{W}_{C}=\delta \mathbf{x}^{T} \mathbf{C} \dot{\mathbf{x}}=\delta \mathbf{x}_{m}^{T} \mathbf{T}^{T} \mathbf{C} \mathbf{T} \dot{\mathbf{x}}_{m}=\delta \mathbf{x}_{m}^{T} \overline{\mathbf{C}} \dot{\mathbf{x}}_{m} \\
\overline{\mathbf{C}}=\mathbf{T}^{T} \mathbf{C} \mathbf{T}=\left[\begin{array}{ll}
\mathbf{I} & -\mathbf{K}_{m s} \mathbf{K}_{s s}^{-1}
\end{array}\right]\left[\begin{array}{cc}
\mathbf{C}_{m m} & \mathbf{C}_{m s} \\
\mathbf{C}_{s m} & \mathbf{C}_{s s}
\end{array}\right]\left[\begin{array}{c}
\mathbf{I} \\
-\mathbf{K}_{s s}^{-1} \mathbf{K}_{s m}
\end{array}\right]= \\
\mathbf{C}_{m m}-\mathbf{K}_{m s} \mathbf{K}_{s s}^{-1} \mathbf{C}_{s m}-\mathbf{C}_{m s} \mathbf{K}_{s s}^{-1} \mathbf{K}_{s m}+\mathbf{K}_{m s} \mathbf{K}_{s s}^{-1} \mathbf{C}_{s s} \mathbf{K}_{s s}^{-1} \mathbf{K}_{s m}
\end{gathered}
$$

Una vez resuelto el problema reducido, se pueden recuperar las coordenadas globales por medio de la matriz de transformación $\mathbf{T}$. La ecuación de equilibrio dinámico del sistema reducido queda del siguiente modo.

$$
\overline{\mathbf{M}} \ddot{\mathbf{x}}_{m}+\overline{\mathbf{C}} \dot{\mathbf{x}}_{m}+\overline{\mathbf{K}} \mathbf{x}_{m}=\overline{\mathbf{P}} f(t)
$$

Respecto a una selección adecuada de los grados de libertad retenidos, hay mucha bibliografía al respecto (Montáns y Muñoz [2013]; Chopra [1995]; Qu [2004]). Dicho aspecto, aunque de gran importancia, queda fuera del alcance de esta tesis. El ejemplo de aplicación elegido se caracteriza por ser un ejemplo sencillo, cuyo problema completo puede ser resuelto de modo relativamente sencillo, de modo que se validará la bondad de la selección de los grados de libertad retenidos.

Las dos reducciones estáticas restantes que se han considerado están definidas en el espacio 
de los estados. En dicho espacio se escribe la ecuación de equilibrio dinámica (Montáns y Muñoz [2013], Chopra [1995]) empleando vectores de estado.

Como ya se ha comentado, el recurso del espacio de los estados surge de la necesidad de desacoplar el sistema de ecuaciones por medio del álgebra compleja al no ser posible desacoplarlo por medio del álgebra lineal. Esta necesidad aparece cuando el amortiguamiento de la estructura no se puede aproximar por medio de un amortiguamiento proporcional a las matrices de masa o rigidez (más específicamente cuando $\mathbf{C}$ y $\mathbf{K}$ son $\mathbf{M}$-conmutativas, i.e. $\mathbf{C M}^{-1} \mathbf{K}=\mathbf{K M}^{-1} \mathbf{C}$, Caughey y O'Kelly [1965]). Esto sucede ante la presencia de dispositivos amortiguadores concentrados en uno o varios grados de libertad de la estructura, diferencias relevantes en las propiedades de amortiguamiento inherentes a diferentes materiales empleados en distintas partes de la estructura, o ante la presencia de elementos rotativos dentro de la estructura con inercias de consideración. El problema se define en estos casos como problema de autovalores complejos o problema de amortiguamiento no clásico. A diferencia del método que se compara en esta tesis (que está en forma estándar), la formulación de los dos métodos de condensación estática que se definen a continuación emplean la forma lineal general de autovalores complejos.

\subsubsection{Condensación estática en el espacio de los estados I (SCSS I)}

El primero de los dos métodos mencionados se obtiene añadiendo al sistema de ecuaciones de equilibrio las ecuaciones dadas por la identidad

$$
\mathbf{M} \dot{\mathbf{x}}-\mathbf{M} \dot{\mathbf{x}}=\mathbf{0},
$$

de modo que se forma el siguiente sistema simétrico de ecuaciones:

$$
\underbrace{\left[\begin{array}{cc}
\mathbf{K} & \mathbf{0} \\
\mathbf{0} & -\mathbf{M}
\end{array}\right]}_{\mathbf{A}^{I}} \underbrace{\left[\begin{array}{c}
\mathbf{x} \\
\dot{\mathbf{x}}
\end{array}\right]}_{\mathbf{X}}+\underbrace{\left[\begin{array}{cc}
\mathbf{C} & \mathbf{M} \\
\mathbf{M} & \mathbf{0}
\end{array}\right]}_{\mathbf{B}^{I}} \underbrace{\left[\begin{array}{c}
\dot{\mathbf{x}} \\
\ddot{\mathbf{x}}
\end{array}\right]}_{\dot{\mathbf{X}}}=\underbrace{\left[\begin{array}{c}
\mathbf{P} f(t) \\
\mathbf{0}
\end{array}\right]}_{\mathbf{F}^{I}}
$$


donde a las matrices del sistema $\mathbf{A}^{I}, \mathbf{B}^{I} \in \mathbb{R}^{2 G \times 2 G}$, siendo $G$ el número de grados de libertad del sistema estructural, se les aplica la partición de "master" y "slave":

$$
\left[\begin{array}{cc}
\mathbf{A}_{m m}^{I} & \mathbf{A}_{m s}^{I} \\
\mathbf{A}_{s m}^{I} & \mathbf{A}_{s s}^{I}
\end{array}\right]\left[\begin{array}{c}
\mathbf{X}_{m} \\
\mathbf{X}_{s}
\end{array}\right]+\left[\begin{array}{cc}
\mathbf{B}_{m m}^{I} & \mathbf{B}_{m s}^{I} \\
\mathbf{B}_{s m}^{I} & \mathbf{B}_{s s}^{I}
\end{array}\right]\left[\begin{array}{c}
\dot{\mathbf{X}}_{m} \\
\dot{\mathbf{X}}_{s}
\end{array}\right]=\left[\begin{array}{c}
\mathbf{F}_{m}^{I} \\
\mathbf{F}_{s}^{I}
\end{array}\right]
$$

cuyas submatrices tienen las siguientes expresiones (por sencillez se emplean subíndices genéri$\cos i, j$, que deben sustituirse por los correspondientes $m, s$ según el cajón correspondiente)

$$
\begin{aligned}
\mathbf{A}_{i j}^{I} & =\left[\begin{array}{cc}
\mathbf{K}_{i j} & \mathbf{0} \\
\mathbf{0} & -\mathbf{M}_{i j}
\end{array}\right] \quad, \quad \mathbf{B}_{i j}^{I}=\left[\begin{array}{cc}
\mathbf{C}_{i j} & \mathbf{M}_{i j} \\
\mathbf{M}_{i j} & \mathbf{0}
\end{array}\right], \quad \mathbf{F}_{i}^{I}=\left[\begin{array}{c}
\mathbf{P}_{i f} f(t) \\
\mathbf{0}
\end{array}\right] \\
\mathbf{X}_{i} & =\left[\begin{array}{c}
\mathbf{X}_{i} \\
\dot{\mathbf{X}}_{i}
\end{array}\right] \quad, \quad \dot{\mathbf{X}}_{i}=\left[\begin{array}{c}
\dot{\mathbf{X}}_{i} \\
\ddot{\mathbf{X}}_{i}
\end{array}\right]
\end{aligned}
$$

Bajo la hipótesis de fuerzas nulas en los grados de libertad "slaves", se obtiene de la segunda fila de la Ec. (3.21):

$$
\begin{aligned}
\mathbf{A}_{s m}^{I} \mathbf{X}_{m}+\mathbf{A}_{s s}^{I} \mathbf{X}_{s}+\mathbf{B}_{s m}^{I} \dot{\mathbf{X}}_{m}+\mathbf{B}_{s s}^{I} \dot{\mathbf{X}}_{s} & =\mathbf{0} \Longrightarrow \\
\mathbf{X}_{s} & =\left(\mathbf{A}_{s s}^{I}\right)^{-1}\left(-\mathbf{A}_{s m}^{I} \mathbf{X}_{m}-\mathbf{B}_{s m}^{I} \dot{\mathbf{X}}_{m}-\mathbf{B}_{s s}^{I} \dot{\mathbf{X}}_{s}\right)
\end{aligned}
$$

Añadiendo la hipótesis adicional de velocidad del vector de estado nula

$$
\dot{\mathbf{X}}=\mathbf{0} \Longrightarrow \dot{\mathbf{X}}_{m}=\mathbf{0}, \dot{\mathbf{X}}_{s}=\mathbf{0}
$$

(que es el motivo por el cual se considera esta reducción dentro del grupo de reducciones estáticas), se obtiene la matriz de cambio de coordenadas $\mathbf{T}^{I}$ :

$$
\mathbf{X}_{s}=-\left(\mathbf{A}_{s s}^{I}\right)^{-1} \mathbf{A}_{s m}^{I} \mathbf{X}_{m} \Longrightarrow \mathbf{T}^{I}=\left[\begin{array}{cc}
\mathbf{I} & \mathbf{0} \\
\mathbf{0} & \mathbf{I} \\
-\mathbf{K}_{s s}^{-1} \mathbf{K}_{s m} & \mathbf{0} \\
\mathbf{0} & -\mathbf{M}_{s s}^{-1} \mathbf{M}_{s m}
\end{array}\right]
$$


de modo que $\quad \mathbf{X}=\left[\begin{array}{c}\mathbf{X}_{m} \\ \mathbf{X}_{s}\end{array}\right]=\mathbf{T}^{I} \mathbf{X}_{m} \quad$ y $\quad \dot{\mathbf{X}}=\mathbf{T}^{I} \dot{\mathbf{X}}_{m}$.

Cabe señalar, que aunque se ha considerado la velocidad del vector de estado nula, el sistema retiene efectos inerciales de modo parcial como se puede observar en la última caja de la matriz de cambio de coordenadas. Usando la siguiente nomenclatura

$$
\mathbf{T}^{I}=\left[\begin{array}{c}
\mathbf{T}_{m}^{I} \\
\mathbf{T}_{s}^{I}
\end{array}\right] \quad, \quad \mathbf{T}_{m}^{I}=\mathbf{I}_{(2 m) \times(2 m)} \quad, \quad \mathbf{T}_{s}^{I}=\left[\begin{array}{cc}
-\mathbf{K}_{s s}^{-1} \mathbf{K}_{s m} & \mathbf{0} \\
\mathbf{0} & -\mathbf{M}_{s s}^{-1} \mathbf{M}_{s m}
\end{array}\right]
$$

el sistema reducido queda definido por las siguientes ecuaciones:

$$
\begin{gathered}
\overline{\mathbf{A}}^{I}=\mathbf{A}_{m m}^{I}+\left(\mathbf{T}_{s}^{I}\right)^{T} \mathbf{A}_{s m}^{I}+\mathbf{A}_{m s}^{I} \mathbf{T}_{s}^{I}+\left(\mathbf{T}_{s}^{I}\right)^{T} \mathbf{A}_{s s}^{I} \mathbf{T}_{s}^{I} \\
\overline{\mathbf{B}}^{I}=\mathbf{B}_{m m}^{I}+\left(\mathbf{T}_{s}^{I}\right)^{T} \mathbf{B}_{s m}^{I}+\mathbf{B}_{m s}^{I} \mathbf{T}_{s}^{I}+\left(\mathbf{T}_{s}^{I}\right)^{T} \mathbf{B}_{s s}^{I} \mathbf{T}_{s}^{I} \\
\overline{\mathbf{F}}^{I}=\mathbf{F}_{m}^{I}+\left(\mathbf{T}_{s}^{I}\right)^{T} \mathbf{F}_{s}^{I}
\end{gathered}
$$

con la siguiente ecuación de equilibrio dinámico

$$
\overline{\mathbf{A}}^{I} \mathbf{X}_{m}+\overline{\mathbf{B}}^{I} \dot{\mathbf{X}}_{m}=\overline{\mathbf{F}}^{I}
$$

Se pueden obtener a partir de las ecuaciones mostradas las matrices de masas, de rigidez y de amortiguamiento del sistema reducido por sustitución. Todas son idénticas a las del modelo de reducción anterior a excepción de la matriz de masas.

\subsubsection{Condensación estática en el espacio de los estados II (SCSS II)}

El último modelo de reducción estático que se propone se obtiene añadiendo al sistema de ecuaciones de equilibrio la ecuación identidad función de la matriz de rigidez:

$$
\mathbf{K} \dot{\mathbf{x}}-\mathbf{K} \dot{\mathbf{x}}=\mathbf{0}
$$


de modo que se forma el siguiente sistema simétrico de ecuaciones:

$$
\underbrace{\left[\begin{array}{cc}
\mathbf{0} & \mathbf{K} \\
\mathbf{K} & \mathbf{C}
\end{array}\right]}_{\mathbf{A}^{I I}} \underbrace{\left[\begin{array}{c}
\mathbf{x} \\
\dot{\mathbf{x}}
\end{array}\right]}_{\mathbf{X}}+\underbrace{\left[\begin{array}{cc}
-\mathbf{K} & \mathbf{0} \\
\mathbf{0} & \mathbf{M}
\end{array}\right]}_{\mathbf{B}^{I I}} \underbrace{\left[\begin{array}{c}
\dot{\mathbf{x}} \\
\ddot{\mathbf{x}}
\end{array}\right]}_{\dot{\mathbf{X}}}=\underbrace{\left[\begin{array}{c}
\mathbf{0} \\
\mathbf{P} f(t)
\end{array}\right]}_{\mathbf{F}^{I I}}
$$

donde a las matrices del sistema $\mathbf{A}^{I I}, \mathbf{B}^{I I} \in \mathbb{R}^{2 G \times 2 G}$, siendo $G$ el número de grados de libertad del sistema estructural, se les aplica la misma partición de "master" y "slave"

$$
\left[\begin{array}{cc}
\mathbf{A}_{m m}^{I I} & \mathbf{A}_{m s}^{I I} \\
\mathbf{A}_{s m}^{I I} & \mathbf{A}_{s s}^{I I}
\end{array}\right]\left[\begin{array}{c}
\mathbf{X}_{m} \\
\mathbf{X}_{s}
\end{array}\right]+\left[\begin{array}{cc}
\mathbf{B}_{m m}^{I I} & \mathbf{B}_{m s}^{I I} \\
\mathbf{B}_{s m}^{I I} & \mathbf{B}_{s s}^{I I}
\end{array}\right]\left[\begin{array}{c}
\dot{\mathbf{X}}_{m} \\
\dot{\mathbf{X}}_{s}
\end{array}\right]=\left[\begin{array}{c}
\mathbf{F}_{m}^{I I} \\
\mathbf{F}_{s}^{I I}
\end{array}\right]
$$

cuyas submatrices tienen las siguientes expresiones (por sencillez se emplean subíndices genéri$\cos i, j$, que deben sustituirse por los correspondientes $m, s$ según el cajón correspondiente)

$$
\begin{aligned}
\mathbf{A}_{i j}^{I I} & =\left[\begin{array}{cc}
\mathbf{0} & \mathbf{K}_{i j} \\
\mathbf{K}_{i j} & \mathbf{C}_{i j}
\end{array}\right], \quad \mathbf{B}_{i j}^{I I}=\left[\begin{array}{cc}
-\mathbf{K}_{i j} & \mathbf{0} \\
\mathbf{0} & \mathbf{M}_{i j}
\end{array}\right], \quad \mathbf{F}_{i}^{I I}=\left[\begin{array}{c}
\mathbf{0} \\
\mathbf{P}_{i f} f(t)
\end{array}\right] \\
\mathbf{X}_{i} & =\left[\begin{array}{c}
\mathbf{X}_{i} \\
\dot{\mathbf{X}}_{i}
\end{array}\right] \quad, \quad \dot{\mathbf{X}}_{i}=\left[\begin{array}{c}
\dot{\mathbf{X}}_{i} \\
\ddot{\mathbf{X}}_{i}
\end{array}\right]
\end{aligned}
$$

Aplicando las mismas hipótesis que en el método anterior, Ec. (3.26) y fuerzas nulas en Ec. (3.36), se obtiene la nueva matriz de cambio de coordenadas $\mathbf{T}^{I I}$ :

$$
\mathbf{X}_{s}=-\left(\mathbf{A}_{s s}^{I I}\right)^{-1} \mathbf{A}_{s m}^{I I} \mathbf{X}_{m} \Longrightarrow \mathbf{T}^{I I}=\left[\begin{array}{cc}
\mathbf{I} & \mathbf{0} \\
\mathbf{0} & \mathbf{I} \\
-\mathbf{K}_{s s}^{-1} \mathbf{K}_{s m} & \mathbf{K}_{s s}^{-1} \mathbf{C}_{s s} \mathbf{K}_{s s}^{-1} \mathbf{K}_{s m}-\mathbf{K}_{s s}^{-1} \mathbf{C}_{s m} \\
\mathbf{0} & -\mathbf{K}_{s s}^{-1} \mathbf{K}_{s m}
\end{array}\right]
$$

de modo que $\quad \mathbf{X}=\left[\begin{array}{c}\mathbf{X}_{m} \\ \mathbf{X}_{s}\end{array}\right]=\mathbf{T}^{I I} \mathbf{X}_{m} \quad$ y $\quad \dot{\mathbf{X}}=\mathbf{T}^{I I} \dot{\mathbf{X}}_{m}$.

A diferencia del método anterior, esta matriz de cambio de coordenadas no retiene efectos 
inerciales. Usando una nomenclatura coherente con la empleada en el método anterior se tiene $\mathbf{T}^{I I}=\left[\begin{array}{c}\mathbf{T}_{m}^{I I} \\ \mathbf{T}_{s}^{I I}\end{array}\right] \quad, \quad \mathbf{T}_{m}^{I I}=\mathbf{I}_{(2 m) \times(2 m)} \quad, \quad \mathbf{T}_{s}^{I I}=\left[\begin{array}{cc}-\mathbf{K}_{s s}^{-1} \mathbf{K}_{s m} & \mathbf{K}_{s s}^{-1} \mathbf{C}_{s s} \mathbf{K}_{s s}^{-1} \mathbf{K}_{s m}-\mathbf{K}_{s s}^{-1} \mathbf{C}_{s m} \\ \mathbf{0} & -\mathbf{K}_{s s}^{-1} \mathbf{K}_{s m}\end{array}\right]$

y el sistema reducido queda definido por las siguientes ecuaciones:

$$
\begin{gathered}
\overline{\mathbf{A}}^{I I}=\mathbf{A}_{m m}^{I I}+\left(\mathbf{T}_{s}^{I I}\right)^{T} \mathbf{A}_{s m}^{I I}+\mathbf{A}_{m s}^{I I} \mathbf{T}_{s}^{I I}+\left(\mathbf{T}_{s}^{I I}\right)^{T} \mathbf{A}_{s s}^{I I} \mathbf{T}_{s}^{I I} \\
\overline{\mathbf{B}}^{I I}=\mathbf{B}_{m m}^{I I}+\left(\mathbf{T}_{s}^{I I}\right)^{T} \mathbf{B}_{s m}^{I I}+\mathbf{B}_{m s}^{I I} \mathbf{T}_{s}^{I I}+\left(\mathbf{T}_{s}^{I I}\right)^{T} \mathbf{B}_{s s}^{I I} \mathbf{T}_{s}^{I I} \\
\overline{\mathbf{F}}^{I I}=\mathbf{F}_{m}^{I I}+\left(\mathbf{T}_{s}^{I I}\right)^{T} \mathbf{F}_{s}^{I I}
\end{gathered}
$$

con la siguiente ecuación de equilibrio dinámico

$$
\overline{\mathbf{A}}^{I I} \mathbf{X}_{m}+\overline{\mathbf{B}}^{I I} \dot{\mathbf{X}}_{m}=\overline{\mathbf{F}}^{I I}
$$

En este caso las matrices de rigidez, amortiguamiento y el vector de cargas coinciden con los descritos en el primer método de condensación estática. La matriz de masas es idéntica a excepción de un término adicional que tiene este tercer método y que es sólo función de las matrices de amortiguamiento y rigidez del sistema.

Por último, cabe señalar que de los 3 métodos indicados, por las hipótesis realizadas en cada uno de ellos (en algún caso contradictorias), los resultados del primero de ellos son mejores que los de los otros dos, siendo el segundo método descrito el que da peores resultados. Se puede encontrar una explicación más detallada de las diferencias en Qu [2004] y más adelante, en el apartado de comparación de resultados. Un aspecto también a tener en cuenta, es que en el caso de amortiguamiento proporcional, en el que no es necesario recurrir a una transformación al espacio modal complejo, la dimensión del problema de autovalores para el método SCDS sería la mitad respecto a los otros dos, con el consiguiente ahorro en tiempo de computación. 


\subsection{Condensación Dinámica}

En esta sección se describen los métodos de condensación dinámica iterativos empleados para la comparación de resultados con el método DACMAM.

El uso de métodos de condensación dinámica iterativos se justifica por una mejora de los resultados a cambio de aumentar el tiempo de computación, aunque los diversos métodos disponibles han ido mejorando ambos aspectos (Paz [1984]; Paz [1989]; Suarez y Singh [1992]; Qu [2004]; Liu y Wu [2011]).

Como ya se ha comentado, se presentan a continuación, de entre todos los disponibles para análisis dinámicos, métodos de reducción que admiten un amortiguamiento no clásico.

Considerando soluciones del estilo $\mathbf{x}=\phi \mathbf{e}^{j \omega t}$, se puede escribir el problema de autovalores correspondiente a la ecuación de equilibrio dinámico, Ec. (3.1), del siguiente modo:

$$
\left(\mathbf{K}+j \omega \mathbf{C}-\omega^{2} \mathbf{M}\right) \boldsymbol{\phi}=\mathbf{0}
$$

Con la partición habitual en grados de libertad "master" y "slave" se tiene

$$
\left[\begin{array}{cc}
\mathbf{K}_{m m}+j \omega \mathbf{C}_{m m}-\omega^{2} \mathbf{M}_{m m} & \mathbf{K}_{m s}+j \omega \mathbf{C}_{m s}-\omega^{2} \mathbf{M}_{m s} \\
\mathbf{K}_{s m}+j \omega \mathbf{C}_{s m}-\omega^{2} \mathbf{M}_{s m} & \mathbf{K}_{s s}+j \omega \mathbf{C}_{s s}-\omega^{2} \mathbf{M}_{s s}
\end{array}\right]\left[\begin{array}{c}
\boldsymbol{\phi}_{m} \\
\boldsymbol{\phi}_{s}
\end{array}\right]=\mathbf{0}
$$

De la segunda ecuación de este sistema se obtiene la matriz de cambio de coordenadas, que define la condensación dinámica para un sistema con amortiguamiento no clásico (condensación dinámica en el espacio de los desplazamientos).

$$
\mathbf{x}=\left[\begin{array}{c}
\mathbf{x}_{m} \\
\mathbf{x}_{s}
\end{array}\right]=\left[\begin{array}{c}
\mathbf{I} \\
-\left(\mathbf{K}_{s s}+j \omega \mathbf{C}_{s s}-\omega^{2} \mathbf{M}_{s s}\right)^{-1}\left(\mathbf{K}_{s m}+j \omega \mathbf{C}_{s m}-\omega^{2} \mathbf{M}_{s m}\right)
\end{array}\right] \mathbf{x}_{m}=\mathbf{T} \mathbf{x}_{m}
$$

Se observa en la ecuación precedente que la resolución de dicha matriz de cambio de coordenadas es compleja por la inversa que contiene. A continuación se describen tres métodos iterativos para aproximar la solución. 


\subsubsection{Condensación dinámica en el espacio de los estados I (DCSS I)}

Se pasa del espacio de los desplazamientos al espacio de los estados añadiendo la identidad de masas, Ec. (3.19), dando lugar a la ecuación de equilibrio dinámico (3.20). El problema de autovalores queda:

$$
\mathbf{A}^{I} \boldsymbol{\Psi}+\mathbf{B}^{I} \boldsymbol{\Psi} \boldsymbol{\Lambda}=\mathbf{0}
$$

donde $\boldsymbol{\Psi}$ es la matriz de autovectores complejos de dimensión $2 G \times 2 G$. La dimensión dobla el número de grados de libertad (gdl) reales al estar compuesta la solución por pares conjugados de autovectores y autovalores. Las condiciones de ortogonalidad para el sistema descrito son

$$
\boldsymbol{\Psi}^{T} \mathbf{A}^{I} \boldsymbol{\Psi}=-\boldsymbol{\Lambda} \quad \mathrm{y} \quad \boldsymbol{\Psi}^{T} \mathbf{B}^{I} \boldsymbol{\Psi}=\mathbf{I}
$$

Con la partición habitual, al considerar sólo los primeros $m$ pares de autovectores conjugados, la Ec. (3.47) queda:

$$
\begin{gathered}
\mathbf{A}^{I} \mathbf{\Psi}_{m}+\mathbf{B}^{I} \boldsymbol{\Psi}_{m} \boldsymbol{\Lambda}_{m}=\mathbf{0} \\
{\left[\begin{array}{cc}
\mathbf{A}_{m m}^{I} & \mathbf{A}_{m s}^{I} \\
\mathbf{A}_{s m}^{I} & \mathbf{A}_{s s}^{I}
\end{array}\right]\left[\begin{array}{c}
\boldsymbol{\Psi}_{m m} \\
\boldsymbol{\Psi}_{s m}
\end{array}\right]+\left[\begin{array}{cc}
\mathbf{B}_{m m}^{I} & \mathbf{B}_{m s}^{I} \\
\mathbf{B}_{s m}^{I} & \mathbf{B}_{s s}^{I}
\end{array}\right]\left[\begin{array}{c}
\boldsymbol{\Psi}_{m m} \\
\boldsymbol{\Psi}_{s m}
\end{array}\right] \boldsymbol{\Lambda}_{m}=\mathbf{0},}
\end{gathered}
$$

donde $\boldsymbol{\Psi}_{s m}$, de dimensión $2 s \times 2 m$, denota los grados de libertad "slaves" en desplazamientos y en velocidades de los primeros $m$ pares de autovectores conjugados; $\boldsymbol{\Lambda}_{m}$, de dimensión $2 m \times 2 m$, es la matriz que contiene en los elementos de su diagonal los autovalores complejos conjugados correspondientes a los $m$ pares de autovectores conjugados. De la segunda ecuación del sistema se obtiene la relación entre los grados de libertad "master" y "slave".

$$
\begin{gathered}
\mathbf{A}_{s m}^{I} \boldsymbol{\Psi}_{m m}+\mathbf{A}_{s s}^{I} \boldsymbol{\Psi}_{s m}+\mathbf{B}_{s m}^{I} \boldsymbol{\Psi}_{m m} \boldsymbol{\Lambda}_{m}+\mathbf{B}_{s s}^{I} \boldsymbol{\Psi}_{s m} \boldsymbol{\Lambda}_{m}=\mathbf{0} \\
\boldsymbol{\Psi}_{s m}=-\left(\mathbf{A}_{s s}^{I}\right)^{-1}\left(\mathbf{A}_{s m}^{I} \boldsymbol{\Psi}_{m m}+\mathbf{B}_{s m}^{I} \boldsymbol{\Psi}_{m m} \boldsymbol{\Lambda}_{m}+\mathbf{B}_{s s}^{I} \boldsymbol{\Psi}_{s m} \boldsymbol{\Lambda}_{m}\right)
\end{gathered}
$$

La matriz de condensación dinámica, $\mathbf{R}^{I}$, que es la caja inferior de la matriz de cambio de 
base, tiene dimensión $2 s \times 2 m$ y se obtiene de la siguiente expresión:

$$
\mathbf{R}^{I}=-\left(\mathbf{A}_{s s}^{I}\right)^{-1}\left[\mathbf{A}_{s m}^{I}+\left(\mathbf{B}_{s m}^{I}+\mathbf{B}_{s s}^{I} \mathbf{R}^{I}\right) \mathbf{\Psi}_{m m} \mathbf{\Lambda}_{m} \mathbf{\Psi}_{m m}^{-1}\right]
$$

Se muestra a continuación el sistema de matrices reducido:

$$
\mathbf{T}=\left[\begin{array}{c}
\mathbf{I} \\
\mathbf{R}^{I}
\end{array}\right] \Longrightarrow \begin{gathered}
\overline{\mathbf{A}}^{I}=\mathbf{A}_{m m}^{I}+\left(\mathbf{R}^{I}\right)^{T} \mathbf{A}_{s m}^{I}+\mathbf{A}_{m s}^{I} \mathbf{R}^{I}+\left(\mathbf{R}^{I}\right)^{T} \mathbf{A}_{s s}^{I} \mathbf{R}^{I} \\
\overline{\mathbf{B}}^{I}=\mathbf{B}_{m m}^{I}+\left(\mathbf{R}^{I}\right)^{T} \mathbf{B}_{s m}^{I}+\mathbf{B}_{m s}^{I} \mathbf{R}^{I}+\left(\mathbf{R}^{I}\right)^{T} \mathbf{B}_{s s}^{I} \mathbf{R}^{I} \\
\overline{\mathbf{F}}^{I}=\mathbf{F}_{m}^{I}+\left(\mathbf{R}^{I}\right)^{T} \mathbf{F}_{s}^{I}
\end{gathered}
$$

Y la ecuación de autovalores del sistema reducido, junto a las condiciones de ortogonalidad:

$$
\overline{\mathbf{A}}^{I} \boldsymbol{\Psi}_{m m}+\overline{\mathbf{B}}^{I} \boldsymbol{\Psi}_{m m} \boldsymbol{\Lambda}_{m}=\mathbf{0} \quad, \quad \boldsymbol{\Psi}_{m m}^{T} \overline{\mathbf{A}}^{I} \boldsymbol{\Psi}_{m m}=-\boldsymbol{\Lambda}_{m} \quad \text { y } \quad \boldsymbol{\Psi}_{m m}^{T} \overline{\mathbf{B}}^{I} \boldsymbol{\Psi}_{m m}=\mathbf{I}
$$

La matriz de condensación dinámica no se puede resolver linealmente, por lo que se puede aproximar su valor de modo iterativo a partir de la Ec. (3.51), donde la matriz de condensación dinámica es sustituida en el término de la derecha, en primera aproximación, por una estimación inicial, y en sucesivas iteraciones por el valor de la iteración anterior.

De las ecuaciones de ortogonalidad, se pueden obtener expresiones de la ecuación que sustituyen las matrices de autovectores y sus inversas por las matrices $\overline{\mathbf{A}}^{I}$ y $\overline{\mathbf{B}}^{I}$. Es importante señalar que estas matrices, al igual que las matrices de autovectores, son función de la matriz de condensación dinámica y se evalúan en cada iteración. La expresión óptima de la matriz de condensación dinámica, para evitar el cálculo de inversas (i.e. evitar la operación de resolver un sistema lineal), queda para la iteración $n$ :

$$
\mathbf{R}_{n}^{I}=-\left(\mathbf{A}_{s s}^{I}\right)^{-1}\left[\mathbf{A}_{s m}^{I}-\left(\mathbf{B}_{s m}^{I}+\mathbf{B}_{s s}^{I} \mathbf{R}_{n-1}^{I}\right) \mathbf{\Psi}_{m m, n-1} \mathbf{\Psi}_{m m, n-1}^{T} \overline{\mathbf{A}}_{n-1}^{I}\right]
$$

donde $\boldsymbol{\Psi}_{m m, n}$ indica la iteración $n$-ésima de la matriz de autovectores del sistema reducido. Del mismo modo, las expresiones para la iteración $n$ de las matrices del sistema reducido quedan:

$$
\begin{aligned}
\overline{\mathbf{A}}_{n}^{I} & =\mathbf{A}_{m m}^{I}+\left(\mathbf{R}_{n}^{I}\right)^{T} \mathbf{A}_{s m}^{I}+\mathbf{A}_{m s}^{I} \mathbf{R}_{n}^{I}+\left(\mathbf{R}_{n}^{I}\right)^{T} \mathbf{A}_{s s}^{I} \mathbf{R}_{n}^{I} \\
\overline{\mathbf{B}}_{n}^{I} & =\mathbf{B}_{m m}^{I}+\left(\mathbf{R}_{n}^{I}\right)^{T} \mathbf{B}_{s m}^{I}+\mathbf{B}_{m s}^{I} \mathbf{R}_{n}^{I}+\left(\mathbf{R}_{n}^{I}\right)^{T} \mathbf{B}_{s s}^{I} \mathbf{R}_{n}^{I}
\end{aligned}
$$


Respecto a la aproximación inicial de la matriz de condensación, el valor inmediato es:

$$
\mathbf{R}_{0}^{I}=-\left(\mathbf{A}_{s s}^{I}\right)^{-1} \mathbf{A}_{s m}^{I}
$$

Otra aproximación que se aplica en la comparación, propuesta por Rivera et al. [1999], reduce el esfuerzo de computación al reducir de dos a una las matrices inversas que es necesario evaluar para obtener la aproximación inicial, además de coincidir con el valor de la matriz de condensación estática en el espacio físico, Ec. (3.10):

$$
\mathbf{R}_{0}^{I}=-\left[\begin{array}{cc}
\mathbf{K}_{s s}^{-1} \mathbf{K}_{s m} & \mathbf{0} \\
\mathbf{0} & \mathbf{K}_{s s}^{-1} \mathbf{K}_{s m}
\end{array}\right]
$$

\subsubsection{Condensación dinámica en el espacio de los estados II (DCSS II)}

Se repite el desarrollo para el espacio de los estados que se obtiene, partiendo del espacio de los desplazamientos, a través de la identidad de rigideces, Ec. (3.33). El sistema corresponde a la Ec. (3.34) y el problema de autovalores tiene la forma:

$$
\mathbf{A}^{I I} \boldsymbol{\Psi}+\mathbf{B}^{I I} \boldsymbol{\Psi} \boldsymbol{\Lambda}=\mathbf{0},
$$

donde $\boldsymbol{\Psi}$ es la matriz de autovectores complejos de dimensión $2 G \times 2 G$. Las condiciones de ortogonalidad para el sistema descrito son iguales al caso anterior:

$$
\boldsymbol{\Psi}^{T} \mathbf{A}^{I I} \boldsymbol{\Psi}=-\boldsymbol{\Lambda} \quad \mathbf{y} \quad \boldsymbol{\Psi}^{T} \mathbf{B}^{I I} \boldsymbol{\Psi}=\mathbf{I}
$$

Con la partición habitual, al considerar sólo los primeros $m$ pares de autovectores conjugados, la Ec. (3.58) queda:

$$
\begin{gathered}
\mathbf{A}^{I I} \mathbf{\Psi}_{m}+\mathbf{B}^{I I} \boldsymbol{\Psi}_{m} \boldsymbol{\Lambda}_{m}=\mathbf{0} \\
{\left[\begin{array}{cc}
\mathbf{A}_{m m}^{I I} & \mathbf{A}_{m s}^{I I} \\
\mathbf{A}_{s m}^{I I} & \mathbf{A}_{s s}^{I I}
\end{array}\right]\left[\begin{array}{c}
\boldsymbol{\Psi}_{m m} \\
\boldsymbol{\Psi}_{s m}
\end{array}\right]+\left[\begin{array}{cc}
\mathbf{B}_{m m}^{I I} & \mathbf{B}_{m s}^{I I} \\
\mathbf{B}_{s m}^{I I} & \mathbf{B}_{s s}^{I I}
\end{array}\right]\left[\begin{array}{c}
\boldsymbol{\Psi}_{m m} \\
\boldsymbol{\Psi}_{s m}
\end{array}\right] \boldsymbol{\Lambda}_{m}=\mathbf{0},}
\end{gathered}
$$

donde $\boldsymbol{\Psi}_{s m}$, de dimensión $2 s \times 2 m$, denota los grados de libertad "slaves" en desplazamientos y en velocidades de los primeros $m$ pares de autovectores conjugados; $\boldsymbol{\Lambda}_{m}$, de dimensión $2 m \times 2 m$, 
es la matriz que contiene en los elementos de su diagonal los autovalores complejos conjugados correspondientes a los $m$ pares de autovectores conjugados. De la segunda ecuación del sistema se obtiene la relación entre los grados de libertad "master" y "slave". La matriz de condensación dinámica, $\mathbf{R}^{I I}$, que se corresponde con la caja inferior de la matriz de cambio de base, tiene dimensión $2 s \times 2 m$ y resulta:

$$
\mathbf{R}^{I I}=-\left(\mathbf{A}_{s s}^{I I}\right)^{-1}\left[\mathbf{A}_{s m}^{I I}+\left(\mathbf{B}_{s m}^{I I}+\mathbf{B}_{s s}^{I I} \mathbf{R}^{I I}\right) \mathbf{\Psi}_{m m} \boldsymbol{\Lambda}_{m} \mathbf{\Psi}_{m m}^{-1}\right]
$$

El sistema de matrices reducido tiene también la misma forma que el caso anterior:

$$
\mathbf{T}=\left[\begin{array}{c}
\mathbf{I} \\
\mathbf{R}^{I I}
\end{array}\right] \Longrightarrow \begin{gathered}
\overline{\mathbf{A}}^{I I}=\mathbf{A}_{m m}^{I I}+\left(\mathbf{R}^{I I}\right)^{T} \mathbf{A}_{s m}^{I I}+\mathbf{A}_{m s}^{I I} \mathbf{R}^{I I}+\left(\mathbf{R}^{I I}\right)^{T} \mathbf{A}_{s s}^{I I} \mathbf{R}^{I I} \\
\overline{\mathbf{B}}^{I I}=\mathbf{B}_{m m}^{I I}+\left(\mathbf{R}^{I I}\right)^{T} \mathbf{B}_{s m}^{I I}+\mathbf{B}_{m s}^{I I} \mathbf{R}^{I I}+\left(\mathbf{R}^{I I}\right)^{T} \mathbf{B}_{s s}^{I I} \mathbf{R}^{I I} \\
\overline{\mathbf{F}}^{I I}=\mathbf{F}_{m}^{I I}+\left(\mathbf{R}^{I I}\right)^{T} \mathbf{F}_{s}^{I I}
\end{gathered}
$$

Y la ecuación del sistema reducido, junto a las condiciones de ortogonalidad:

$$
\overline{\mathbf{A}}^{I I} \boldsymbol{\Psi}_{m m}+\overline{\mathbf{B}}^{I I} \boldsymbol{\Psi}_{m m} \boldsymbol{\Lambda}_{m}=\mathbf{0} \quad, \quad \boldsymbol{\Psi}_{m m}^{T} \overline{\mathbf{A}}^{I I} \boldsymbol{\Psi}_{m m}=-\boldsymbol{\Lambda}_{m} \quad \text { y } \quad \boldsymbol{\Psi}_{m m}^{T} \overline{\mathbf{B}}^{I I} \boldsymbol{\Psi}_{m m}=\mathbf{I}
$$

A partir de la Ec. (3.61), introduciendo la condición de ortogonalidad dada por la Ec. (3.63), se obtiene la expresión de la matriz de condensación dinámica para la iteración $n$ :

$$
\mathbf{R}_{n}^{I I}=-\left(\mathbf{A}_{s s}^{I I}\right)^{-1}\left[\mathbf{A}_{s m}^{I I}-\left(\mathbf{B}_{s m}^{I I}+\mathbf{B}_{s s}^{I I} \mathbf{R}_{n-1}^{I I}\right) \mathbf{\Psi}_{m m, n-1} \mathbf{\Psi}_{m m, n-1}^{T} \overline{\mathbf{A}}_{n-1}^{I I}\right]
$$

Respecto a la aproximación inicial de la matriz de condensación, se emplea:

$$
\mathbf{R}_{0}^{I I}=-\left(\mathbf{A}_{s s}^{I I}\right)^{-1} \mathbf{A}_{s m}^{I I}
$$

\subsubsection{Condensación dinámica en el espacio de los estados III (DCSS III)}

Se emplea, para completar la comparación, un espacio de estados al que se llega combinando la Ec. (3.1) y su derivada, que da lugar al siguiente problema de autovalores:

$$
\mathbf{A}^{I I I} \mathbf{\Psi}+\mathbf{B}^{I I I} \mathbf{\Psi} \mathbf{\Lambda}^{2}=\mathbf{0}
$$


donde $\quad \mathbf{A}^{I I I}=\mathbf{A}^{I I} \quad, \quad \mathbf{B}^{I I I}=\mathbf{B}^{I} \quad, \quad \mathbf{F}^{I I I}=\left[\begin{array}{c}\mathbf{P} \dot{f}(t) \\ \mathbf{P} f(t)\end{array}\right]=\mathbf{F}^{I I}+\dot{\mathbf{F}}^{I}$, y $\mathbf{\Psi}$ es la matriz de autovectores complejos de dimensión $2 G \times 2 G$. Las condiciones de ortogonalidad para el sistema descrito difieren de los casos anteriores:

$$
\boldsymbol{\Psi}^{T} \mathbf{A}^{I I I} \boldsymbol{\Psi}=-\boldsymbol{\Lambda}^{2} \quad \text { y } \quad \boldsymbol{\Psi}^{T} \mathbf{B}^{I I I} \boldsymbol{\Psi}=\mathbf{I}
$$

Con la partición habitual, al considerar sólo los primeros $m$ pares de autovectores conjugados, la Ec. (3.66) queda:

$$
\begin{gathered}
\mathbf{A}^{I I I} \mathbf{\Psi}_{m}+\mathbf{B}^{I I I} \mathbf{\Psi}_{m} \boldsymbol{\Lambda}_{m}^{2}=\mathbf{0} \\
{\left[\begin{array}{ll}
\mathbf{A}_{m m}^{I I I} & \mathbf{A}_{m s}^{I I I} \\
\mathbf{A}_{s m}^{I I I} & \mathbf{A}_{s s}^{I I I}
\end{array}\right]\left[\begin{array}{c}
\boldsymbol{\Psi}_{m m} \\
\boldsymbol{\Psi}_{s m}
\end{array}\right]+\left[\begin{array}{ll}
\mathbf{B}_{m m}^{I I I} & \mathbf{B}_{m s}^{I I I} \\
\mathbf{B}_{s m}^{I I I} & \mathbf{B}_{s s}^{I I I}
\end{array}\right]\left[\begin{array}{c}
\boldsymbol{\Psi}_{m m} \\
\boldsymbol{\Psi}_{s m}
\end{array}\right] \boldsymbol{\Lambda}_{m}^{2}=\mathbf{0},}
\end{gathered}
$$

donde $\boldsymbol{\Psi}_{s m}$, de dimensión $2 s \times 2 m$, denota los grados de libertad "slaves" en desplazamientos y en velocidades de los primeros $m$ pares de autovectores conjugados; $\boldsymbol{\Lambda}_{m}^{2}$, de dimensión $2 m \times 2 m$, es la matriz que contiene en los elementos de su diagonal el cuadrado de los autovalores complejos conjugados correspondientes a los $m$ pares de autovectores conjugados. De la segunda ecuación del sistema se obtiene la relación entre los grados de libertad "master" y "slave". La matriz de condensación dinámica, $\mathbf{R}^{I I I}$, que corresponde a la caja inferior de la matriz de cambio de base, tiene dimensión $2 s \times 2 m$ y resulta:

$$
\mathbf{R}^{I I I}=-\left(\mathbf{A}_{s s}^{I I I}\right)^{-1}\left[\mathbf{A}_{s m}^{I I I}+\left(\mathbf{B}_{s m}^{I I I}+\mathbf{B}_{s s}^{I I I} \mathbf{R}^{I I I}\right) \mathbf{\Psi}_{m m} \boldsymbol{\Lambda}_{m}^{2} \mathbf{\Psi}_{m m}^{-1}\right]
$$

El sistema de matrices reducido en este tercer espacio queda:

$$
\mathbf{T}^{I I I}=\left[\begin{array}{c}
\mathbf{I} \\
\mathbf{R}^{I I I}
\end{array}\right] \Longrightarrow \begin{gathered}
\overline{\mathbf{A}}^{I I I}=\mathbf{A}_{m m}^{I I I}+\left(\mathbf{R}^{I I I}\right)^{T} \mathbf{A}_{s m}^{I I I}+\mathbf{A}_{m s}^{I I I} \mathbf{R}^{I I I}+\left(\mathbf{R}^{I I I}\right)^{T} \mathbf{A}_{s s}^{I I I} \mathbf{R}^{I I I} \\
\overline{\mathbf{B}}^{I I I}=\mathbf{B}_{m m}^{I I I}+\left(\mathbf{R}^{I I I}\right)^{T} \mathbf{B}_{s m}^{I I I}+\mathbf{B}_{m s}^{I I I} \mathbf{R}^{I I I}+\left(\mathbf{R}^{I I I}\right)^{T} \mathbf{B}_{s s}^{I I I} \mathbf{R}^{I I I} \\
\overline{\mathbf{F}}^{I I I}=\mathbf{F}_{m}^{I I I}+\left(\mathbf{R}^{I I I}\right)^{T} \mathbf{F}_{s}^{I I I}
\end{gathered}
$$

La ecuación del sistema reducido, junto a las condiciones de ortogonalidad:

$$
\overline{\mathbf{A}}^{I I I} \boldsymbol{\Psi}_{m m}+\overline{\mathbf{B}}^{I I I} \boldsymbol{\Psi}_{m m} \boldsymbol{\Lambda}_{m}^{2}=\mathbf{0} \quad, \quad \boldsymbol{\Psi}_{m m}^{T} \overline{\mathbf{A}}^{I I I} \boldsymbol{\Psi}_{m m}=-\boldsymbol{\Lambda}_{m}^{2} \quad \text { y } \quad \boldsymbol{\Psi}_{m m}^{T} \overline{\mathbf{B}}^{I I I} \boldsymbol{\Psi}_{m m}=\mathbf{I}
$$


A partir de la Ec. (3.69), introduciendo la condición de ortogonalidad dada por la Ec. (3.71), se obtiene la expresión de la matriz de condensación dinámica para la iteración $n$ :

$$
\mathbf{R}_{n}^{I I I}=-\left(\mathbf{A}_{s s}^{I I I}\right)^{-1}\left[\mathbf{A}_{s m}^{I I I}-\left(\mathbf{B}_{s m}^{I I I}+\mathbf{B}_{s s}^{I I I} \mathbf{R}_{n-1}^{I I I}\right) \mathbf{\Psi}_{m m, n-1} \mathbf{\Psi}_{m m, n-1}^{T} \overline{\mathbf{A}}_{n-1}^{I I I}\right]
$$

Para la aproximación inicial de la matriz de condensación, se emplea:

$$
\mathbf{R}_{0}^{I I I}=-\left(\mathbf{A}_{s s}^{I I I}\right)^{-1} \mathbf{A}_{s m}^{I I I}
$$

Se combinan las ecuaciones de este espacio con las de los espacios descritos anteriormente para añadir dos métodos adicionales a la comparación como propone Qu [2004] . A estos métodos los denominaremos condensaciones dinámicas del espacio de los estados I o II híbridos con el espacio III (DCSS I y DCSS II híbridos con la matriz de cambio de coordenadas al espacio de los estados III según el caso). La combinación consiste en aplicar la matriz de cambio de coordenadas $\mathbf{T}^{I I I}$ a los sistemas con la Ec. (3.20) y la Ec. (3.34). En este caso los términos $n$-ésimos de las matrices dinámicas y del sistema quedan del siguiente modo:

$$
\begin{aligned}
\mathbf{R}_{n}^{I I I}=- & \left(\mathbf{A}_{s s}^{I I I}\right)^{-1}\left[\mathbf{A}_{s m}^{I I I}-\left(\mathbf{B}_{s m}^{I I I}+\mathbf{B}_{s s}^{I I I} \mathbf{R}_{n-1}^{I I I}\right) \mathbf{\Psi}_{m m, n-1} \mathbf{\Lambda}_{m, n-1} \mathbf{\Psi}_{m m, n-1}^{T} \overline{\mathbf{A}}_{n-1}^{I}\right] \\
& \overline{\mathbf{A}}_{n}^{I}=\mathbf{A}_{m m}^{I}+\left(\mathbf{R}_{n}^{I I I}\right)^{T} \mathbf{A}_{s m}^{I}+\mathbf{A}_{m s}^{I} \mathbf{R}_{n}^{I I I}+\left(\mathbf{R}_{n}^{I I I}\right)^{T} \mathbf{A}_{s s}^{I I} \mathbf{R}_{n}^{I I I} \\
& \overline{\mathbf{B}}_{n}^{I I}=\mathbf{B}_{m m}^{I I}+\left(\mathbf{R}_{n}^{I I I}\right)^{T} \mathbf{B}_{s m}^{I}+\mathbf{B}_{m s}^{I} \mathbf{R}_{n}^{I I I}+\left(\mathbf{R}_{n}^{I I I}\right)^{T} \mathbf{B}_{s s}^{I} \mathbf{R}_{n}^{I I I} \\
\mathbf{R}_{n}^{I I I}=- & \left(\mathbf{A}_{s s}^{I I I}\right)^{-1}\left[\mathbf{A}_{s m}^{I I I}-\left(\mathbf{B}_{s m}^{I I I}+\mathbf{B}_{s s}^{I I I} \mathbf{R}_{n-1}^{I I I}\right) \mathbf{\Psi}_{m m, n-1} \mathbf{\Lambda}_{m, n-1} \mathbf{\Psi}_{m m, n-1}^{T} \overline{\mathbf{A}}_{n-1}^{I I}\right] \\
& \overline{\mathbf{A}}_{n}^{I I}=\mathbf{A}_{m m}^{I I}+\left(\mathbf{R}_{n}^{I I I}\right)^{T} \mathbf{A}_{s m}^{I I}+\mathbf{A}_{m s}^{I I} \mathbf{R}_{n}^{I I I}+\left(\mathbf{R}_{n}^{I I I}\right)^{T} \mathbf{A}_{s s}^{I I} \mathbf{R}_{n}^{I I I} \\
& \overline{\mathbf{B}}_{n}^{I I}=\mathbf{B}_{m m}^{I I}+\left(\mathbf{R}_{n}^{I I I}\right)^{T} \mathbf{B}_{s m}^{I I}+\mathbf{B}_{m s}^{I I} \mathbf{R}_{n}^{I I I}+\left(\mathbf{R}_{n}^{I I I}\right)^{T} \mathbf{B}_{s s}^{I I} \mathbf{R}_{n}^{I I I}
\end{aligned}
$$




\section{Capítulo 4}

\section{PRIMERAS CONSIDERACIONES}

\section{RESPECTO A LA}

\section{COMPARACIÓN DE LOS MÉTODOS}

\subsection{Matrices en el espacio físico no simétricas}

Se debe notar que los métodos de reducción indicados hasta ahora parten de la hipótesis, no siempre cierta, de que las matrices de amortiguamiento y de rigidez en el espacio físico son simétricas, y como tal se han obtenido las condiciones de ortogonalidad. Trabajar en el espacio simétrico tiene ventajas en tiempos de computación al no requerir resolver un sistema lineal y un problema de autovalores adicionales, cuya solución es necesaria si las matrices implicadas en el problema de resolución de autovalores no son simétricas. Las hipótesis de simetría, aunque se cumplen en general, no son ciertas en el caso de que las estructuras tengan un control activo, en el caso de microdinámica o matrices de amortiguamiento obtenidas de experimentos (Udwadia [2009]).

En el caso de que no lo fueran, se pueden ampliar los métodos de condensación para contemplar dicha posibilidad (Rao [2002]), siendo el sistema resultante notablemente más complejo, 
como se muestra a continuación.

Si las matrices $A$ y $B$ de las condensaciones dinámicas son no simétricas, se deben resolver dos problemas de autovalores:

$$
\mathbf{A} \boldsymbol{\Psi}+\mathbf{B} \boldsymbol{\Psi} \boldsymbol{\Lambda}=\mathbf{0} \quad \text { y } \quad \mathbf{A}^{T} \boldsymbol{\Gamma}+\mathbf{B}^{T} \boldsymbol{\Lambda}=\mathbf{0}
$$

y las condiciones de ortogonalidad quedan de la forma

$$
\boldsymbol{\Gamma}^{T} \mathbf{A} \Psi=-\boldsymbol{\Lambda} \quad \text { y } \quad \boldsymbol{\Gamma}^{T} \mathbf{B} \Psi=\mathbf{I}
$$

siendo las columnas de $\boldsymbol{\Gamma}$ los autovectores del sistema traspuesto o autovectores del lado izquierdo. Aplicando un razonamiento análogo al de las condensaciones dinámicas, se tienen la matriz de condensación dinámica para el lado derecho (se usarán los índices $R$ para derecho y $L$ para izquierdo)

$$
\mathbf{R}^{R}=-\mathbf{A}_{s s}^{-1}\left[\mathbf{A}_{s m}+\left(\mathbf{B}_{s m}+\mathbf{B}_{s s} \mathbf{R}^{R}\right) \mathbf{\Psi}_{m m} \boldsymbol{\Lambda}_{m} \mathbf{\Psi}_{m m}^{-1}\right]
$$

y para el lado izquierdo

$$
\mathbf{R}^{L}=-\mathbf{A}_{s s}^{-T}\left[\mathbf{A}_{s m}^{T}+\left(\mathbf{B}_{s m}^{T}+\mathbf{B}_{s s}^{T} \mathbf{R}^{L}\right) \boldsymbol{\Gamma}_{m m} \boldsymbol{\Lambda}_{m} \boldsymbol{\Gamma}_{m m}^{-1}\right]
$$

Se tienen por tanto dos sistemas de ecuaciones implícitas que requieren de dos iteraciones para resolver cada uno de ellos. Se elige la misma expresión que en los apartados anteriores, la que requiere menos esfuerzo de computación, para evaluar las matrices de condensación en la iteración $n$ :

$$
\begin{aligned}
& \mathbf{R}_{n}^{R}=-\mathbf{A}_{s s}^{-1}\left[\mathbf{A}_{s m}-\left(\mathbf{B}_{s m}+\mathbf{B}_{s s} \mathbf{R}_{n-1}^{R}\right) \mathbf{\Psi}_{m m, n-1} \mathbf{\Psi}_{m m, n-1}^{T} \overline{\mathbf{A}}_{n-1}\right] \\
& \mathbf{R}_{n}^{L}=-\mathbf{A}_{s s}^{-T}\left[\mathbf{A}_{s m}^{T}-\left(\mathbf{B}_{s m}^{T}+\mathbf{B}_{s s}^{T} \mathbf{R}_{n-1}^{L}\right) \boldsymbol{\Gamma}_{m m, n-1} \boldsymbol{\Gamma}_{m m, n-1}^{T} \overline{\mathbf{A}}_{n-1}\right]
\end{aligned}
$$

Las matrices del sistema reducido, $\overline{\mathbf{A}}$ y $\overline{\mathbf{B}}$, tienen un valor para cada iteración, con la 
siguiente expresión:

$$
\begin{aligned}
\overline{\mathbf{A}}_{n} & =\mathbf{A}_{m m}+\left(\mathbf{R}_{n}^{L}\right)^{T} \mathbf{A}_{s m}^{I}+\mathbf{A}_{m s}^{I} \mathbf{R}_{n}^{R}+\left(\mathbf{R}_{n}^{L}\right)^{T} \mathbf{A}_{s s}^{I} \mathbf{R}_{n}^{R} \\
\overline{\mathbf{B}}_{n} & =\mathbf{B}_{m m}+\left(\mathbf{R}_{n}^{L}\right)^{T} \mathbf{B}_{s m}^{I}+\mathbf{B}_{m s}^{I} \mathbf{R}_{n}^{R}+\left(\mathbf{R}_{n}^{L}\right)^{T} \mathbf{B}_{s s}^{I} \mathbf{R}_{n}^{R}
\end{aligned}
$$

También se requieren dos valores iniciales para dichas iteraciones, como pueden ser, también de manera análoga a lo descrito para las condensaciones dinámicas con sistemas simétricos $\mathbf{R}_{0}^{R}=-\mathbf{A}_{s s}^{-1} \mathbf{A}_{s m} \quad \mathrm{y} \quad \mathbf{R}_{0}^{L}=-\mathbf{A}_{s s}^{-T} \mathbf{A}_{s m}^{T}$.

Para el método DACMAM, se ha planteado intencionadamente el espacio de los estados no simétrico, que admite matrices de amortiguamiento o de rigidez no simétricas. El tiempo adicional que implica trabajar en un espacio no simétrico no es relevante para resolver sistemas en los que se retienen un número reducido de modos o para el tamaño de las parametrizaciones empleadas habitualmente en la resolución de problemas dinámicos, máxime teniendo en cuenta que los tiempos totales de análisis proporcionados por el método son muy reducidos. En todo caso, la adaptación del método a un espacio simétrico resulta trivial si no se cumpliese alguna de las condiciones mencionadas que hacen irrelevante el incremento de tiempo de computación por trabajar en un espacio no simétrico.

Por último, es de interés señalar que algoritmos recientes de obtención de autovalores y autovectores, en constante desarrollo para afrontar problemas de mayores dimensiones de modo cada vez más eficiente, incluyen la opción de calcular al mismo tiempo los autovectores del lado derecho y los autovectores del lado izquierdo (MATLAB [2014]).

\subsection{Limitaciones del método de aceleración $D A C M A M$ pro- puesto}

Una limitación que tiene el método $D A C M A M$ es la necesidad de retener todos los grados de libertad que estén sometidos a cargas o condiciones de contorno que no estén en equilibrio. En el caso de los métodos de condensación descritos, debido a que el vector de cargas del sistema reducido contiene la información de los grados de libertad descartados, esto no supone mayor complicación.

Para los casos de cargas concentradas esto no supone ninguna desventaja, pero en el caso de 
que el problema soporte cargas inerciales, desplazamientos o velocidades iniciales distribuidas en una zona amplia de grados de libertad o en la estructura completa, implica retener los grados de libertad afectados.

Esto, aunque pudiese parecer lo contrario, no supone un aumento relevante en el tiempo de computación que se requiere, sino que el mayor coste reside en el volumen de datos necesarios para almacenar los grados de libertad sujetos a una excitación para todos los modos considerados.

El hecho de no suponer un incremento de tiempo de computación importante se debe a que la información de cada modo correspondiente a los grados de libertad sujetos a una excitación se emplea únicamente para el cálculo del vector de cargas en el espacio modal real. Una vez realizada esta transformación, no es necesario emplear adicionalmente dicha información si no se requiere conocer la respuesta del sistema en dichos grados de libertad. En todo caso, la dimensión del espacio modal, como se puede ver en la descripción del método (grupo de modos Considerado), es independiente del número de grados de libertad considerados.

Por otro lado, si el problema implica modificaciones en un número elevado de grados de libertad, por ejemplo del orden del número de grados de libertad del problema completo, la resolución de la respuesta dinámica de la estructura por medio del método DACMAM estaría muy penalizada en tiempo de computación debido a la necesidad de retener todos los grados de libertad implicados en las modificaciones, y aumentaría consecuentemente el número de operaciones para trasladar el efecto de dichas modificaciones al espacio modal real. Esta limitación está implícita en la descripción del método $D A C M A M$, ya que está indicado para casos en los que el número de modificaciones es varios órdenes menor que el número de grados de libertad del sistema.

También se debe tener en cuenta que esta situación no es habitual en ingeniería de estructuras, puesto que las modificaciones estructurales para modificar la respuesta dinámica de una estructura o para simular la presencia de un daño se suelen limitar a localizaciones concretas.

Por último, el método DACMAM realiza dos transformaciones de espacios mientras que el resto de métodos empleados sólo una. Esto tiene una pequeña incidencia en el error numérico acumulado en dichas transformaciones y que no se puede evitar, pero es un error del orden del error de máquina y se puede considerar despreciable frente al resto de errores presentes: 
discretización de la estructura, aproximación de geometría y propiedades de material, truncatura modal, etc. Se puede comprobar en los siguientes capítulos cómo este error no es apreciable en los resultados que se muestran. 


\section{Capítulo 5}

\section{CASO DE ESTUDIO PARA LA COMPARACIÓN DE MÉTODOS DE REDUCCIÓN DE MODELOS}

\subsection{Descripción del ejemplo}

En la presentación del método DACMAM (Herrada-Martín [2016]), ya se mostraba una aplicación sobre una gran estructura, el ala de una aeronave no tripulada, dando datos sobre el ahorro de tiempos de análisis y del volumen de datos generados. Esta tesis se ha centrado en la comparación de dicho método con otros, para lo que se ha seleccionado un ejemplo de un edificio ya empleado por otros autores (Wu et al. [1998]; Qu [2004]) para la comparación de métodos de reducción y que se puede ver en la figura (5-1). 


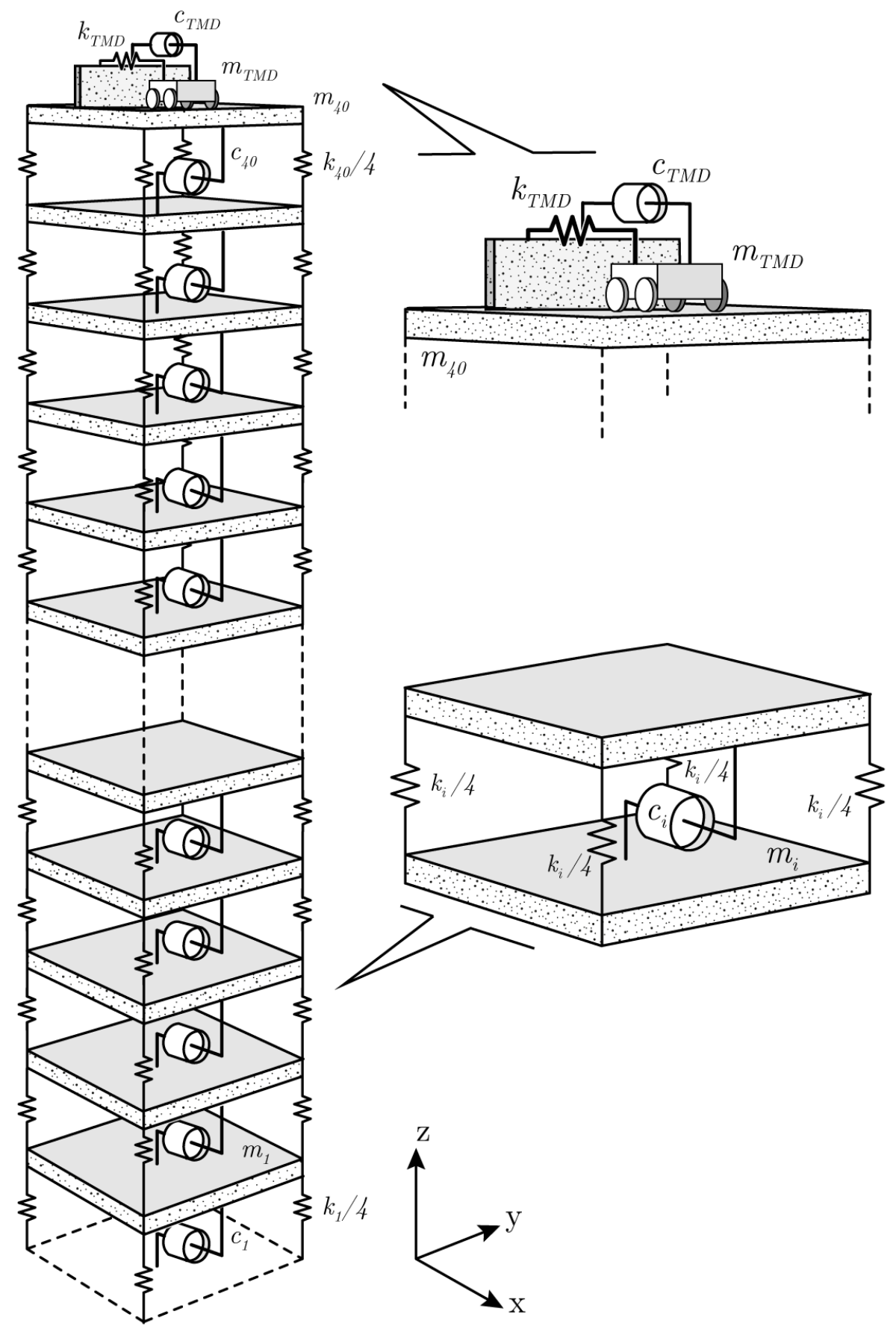

Figura 5-1: Esquema estructural del edificio con 40 plantas y un TMD. 
Este ejemplo tiene como ventaja principal que se trata de un caso sencillo, cuya resolución del problema completo no implica enormes tiempos de computación ni gestionar ficheros de gran tamaño, y sobre el que se tiene un control exhaustivo de todos los parámetros involucrados. Se trata de una estructura con un amortiguamiento no clásico, por lo que permite determinar de manera eficiente los límites del método $D A C M A M$. Y por último, es susceptible de incluir múltiples parametrizaciones que simulen casos prácticos de ingeniería como se verá en los siguientes apartados. A pesar de lo reducido del modelo, se han tomado todas las precauciones para una correcta extrapolación de los resultados obtenidos a modelos de gran tamaño.

El caso a analizar es un edificio que tiene cuarenta plantas, con una masa igual en cada planta, $m_{i}=1,290 \cdot 10^{6} \mathrm{~kg}$, una rigidez de los pilares a cargas transversales igual en cada planta, $k_{i}=10^{9} \mathrm{~N} / \mathrm{m}$, y un amortiguamiento según dirección transversal igual en cada planta, $\quad c_{i}=1,4260 \cdot 10^{7} \mathrm{Ns} / \mathrm{m}$. Adicionalmente, en la última planta hay un dispositivo masaamortiguador sintonizado o tunned mass damper (TMD) con las siguientes especificaciones: $m_{T M D}=0,258 \cdot 10^{6} \mathrm{~kg}, \quad k_{T M D}=300900 \mathrm{~N} / \mathrm{m}, \quad c_{T M D}=83592 \mathrm{Ns} / \mathrm{m}$.

La planta baja se considera empotrada. Para modelar la estructura, se considera un nodo por planta y el nodo del TMD (tunned mass damper, dispositivo amortiguador de vibraciones sintonizado en este caso a la frecuencia del primer modo), y se tiene en cuenta un único grado de libertad por nodo. Dicho grado de libertad es en dirección transversal, se escoge la dirección $x$, siendo $z$ la dirección correspondiente a la altura del edificio, e $y$ formando un triedro a derechas. El mismo modelo se podría emplear para la dirección y (dirección perpendicular en el plano de cada piso).

Se muestran también, en las las figuras (5-2, 5-3, 5-4), los primeros modos propios del edificio con el TMD. Estos serán los modos que se emplearán como base de la comparación de los diferentes modelos en la tesis.

Adicionalmente, se estudiará la modificación de la respuesta estructural del edificio ante cargas dinámicas contemplando diversas modificaciones: amortiguamiento del TMD situado en la última planta para minimizar la respuesta en aceleraciones o desplazamientos de dicha planta, variaciones de masas no estructurales dentro de las últimas plantas del edificio, o variaciones de rigideces simulando el efecto de refuerzos con cruces de San Andrés en algunas de las plantas bajas del edificio. 

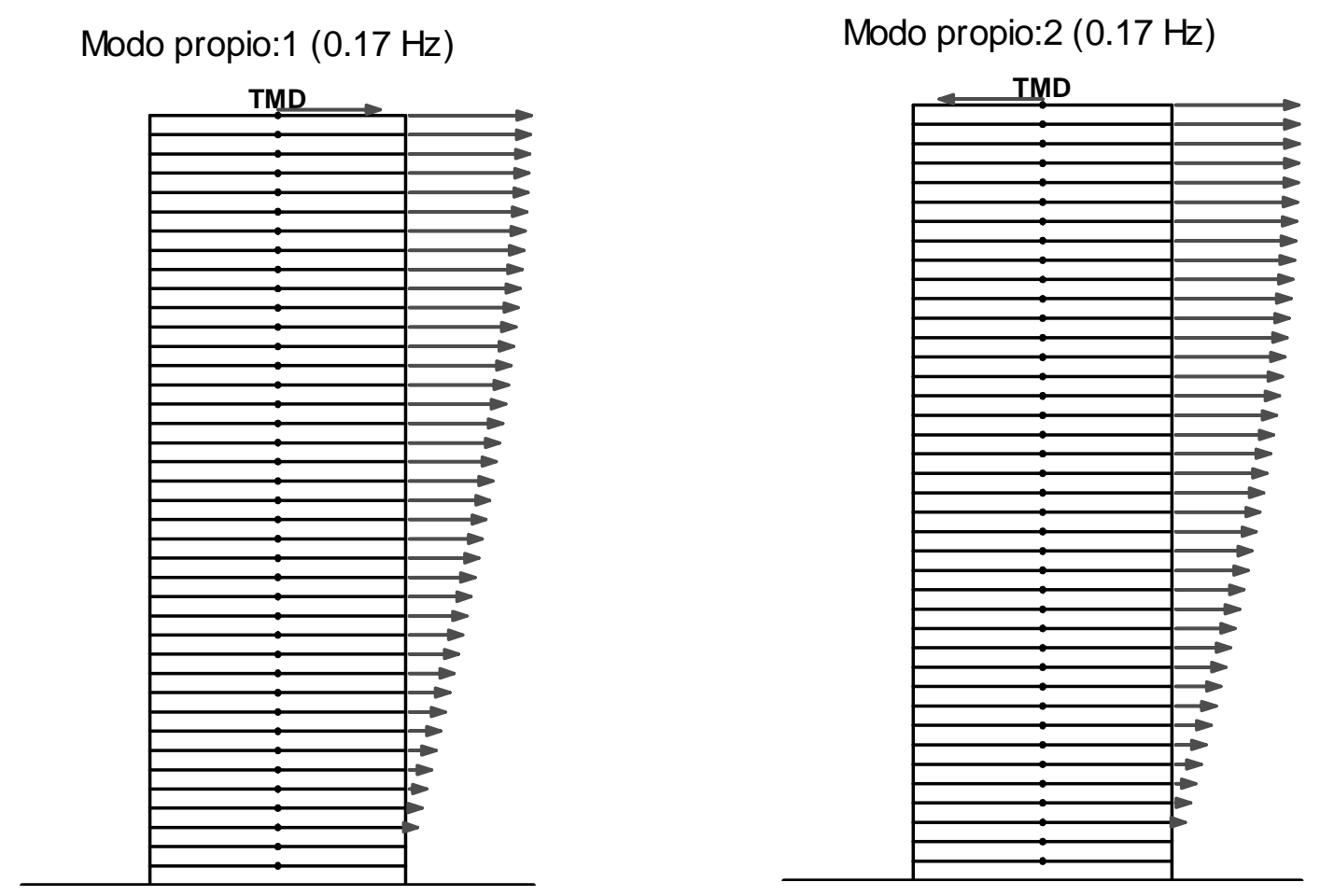

Figura 5-2: Modos propios 1 y 2 del edificio.
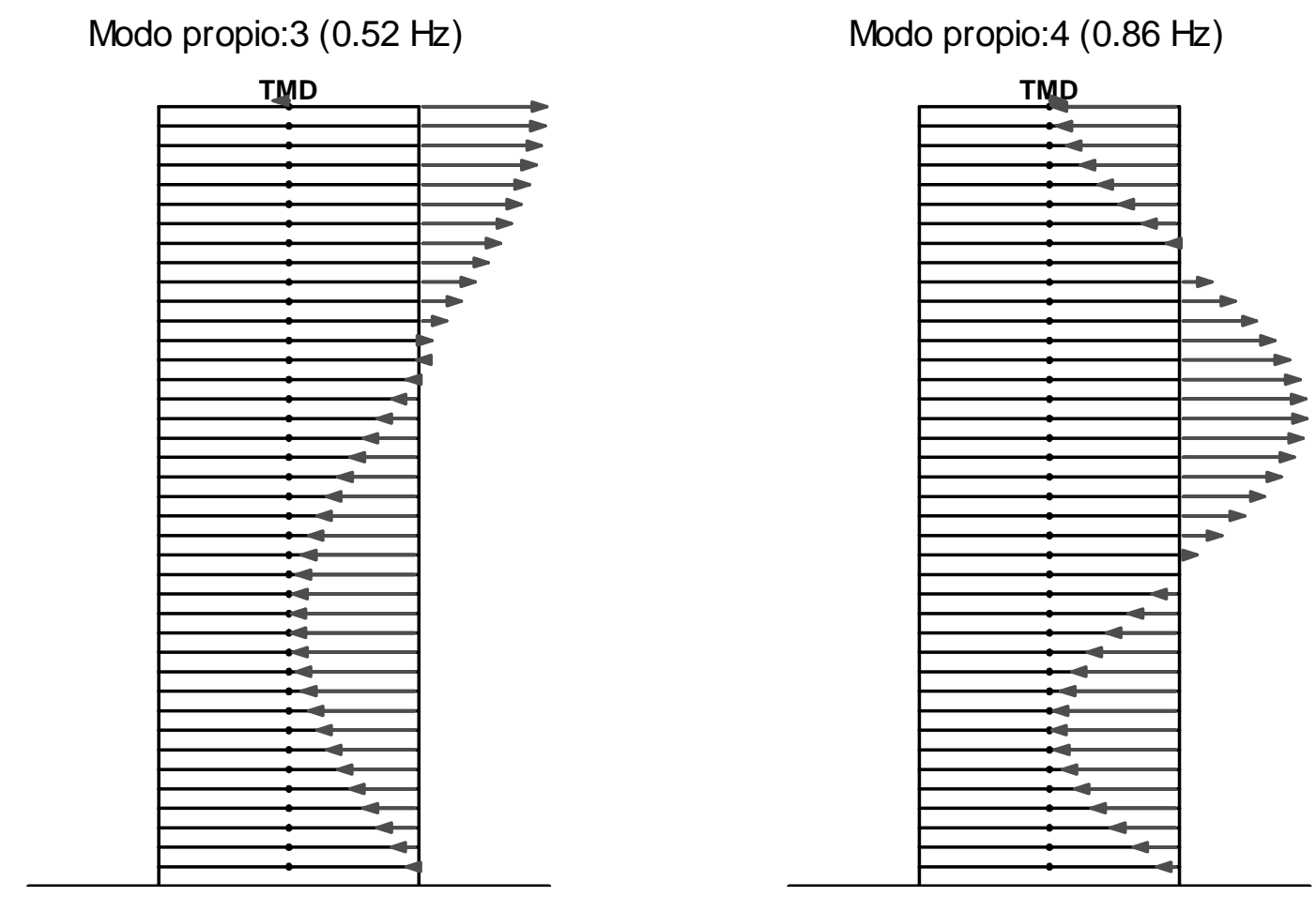

Figura 5-3: Modos propios 3 y 4 del edificio. 

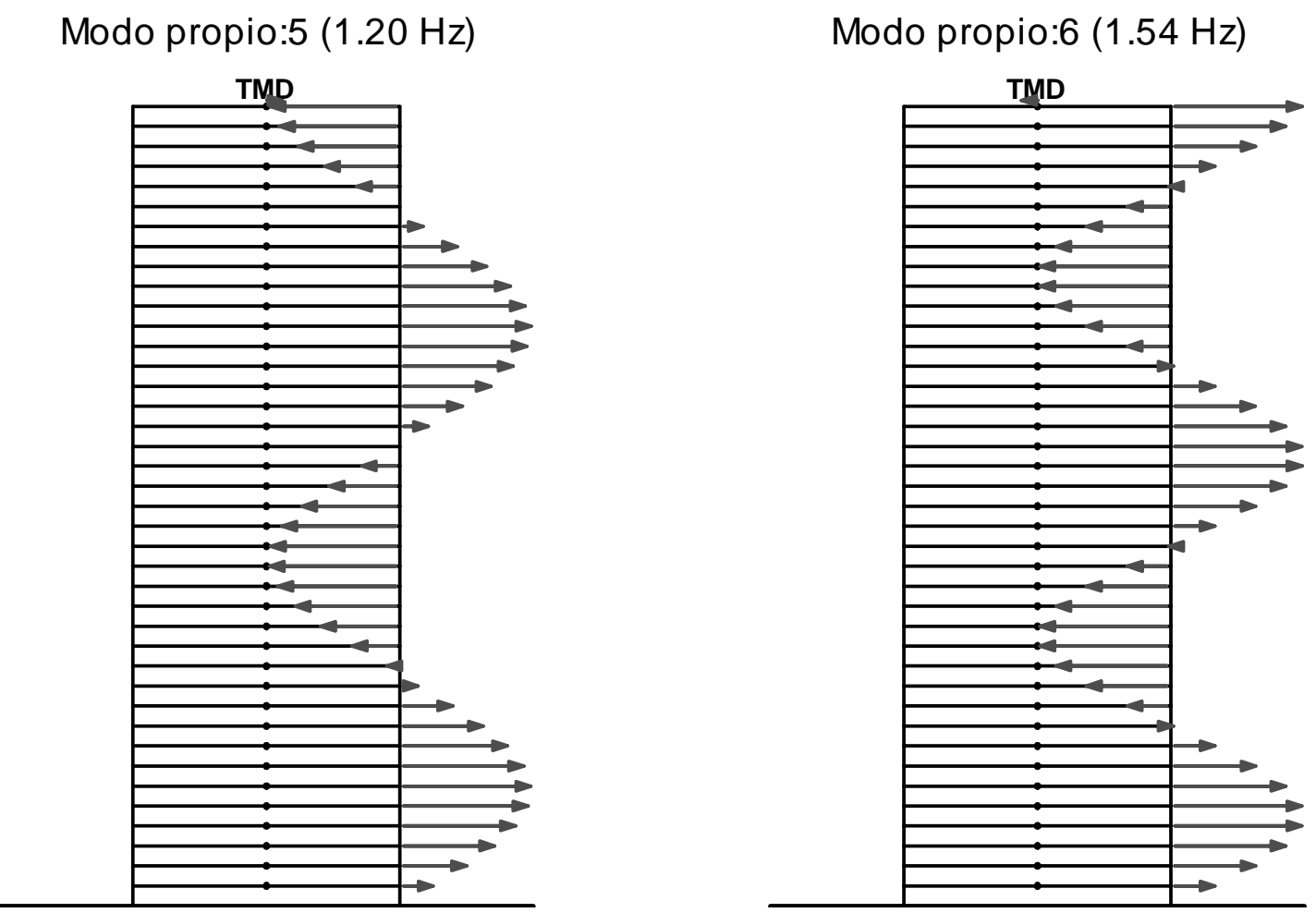

Figura 5-4: Modos propios 5 y 6 del edificio. 


\subsection{Cargas sobre la estructura}

En esta tesis se selecciona un terremoto como carga para la comparación de resultados de los diferentes modelos de reducción ante cargas dinámicas.

Existen muchos lugares en La Tierra en los que los terremotos son frecuentes, y por lo tanto las estructuras civiles deben estar preparadas para soportar las cargas características de los terremotos de dichas localizaciones. Los terremotos presentan características propias en términos de amplitud, duración y contenido en frecuencia dependiendo del lugar donde suceden. Estas características dependen de la localización y orientación de la estructura respecto de las fallas geológicas, del número de éstas, de las características del terreno que las ondas del terremoto atraviesan hasta llegar a la localización exacta de la estructura, etc. Las zonas con alta actividad sísmica tienen habitualmente registros históricos de los terremotos que dan información de la aceleración del terreno durante los mismos y del contenido en frecuencia. Si no se disponen de dichos registros, también hay métodos para generar espectros artificiales de acuerdo a las características geológicas de la zona (Hays [1980]). Las autoridades competentes de dichas regiones con alta actividad sísmica exigen, cada vez con más precisión y alcance, una justificación de resistencia de los edificios y estructuras civiles que se construyen. En España la normativa al respecto está recogida en el Código Técnico de Edificación (CTE), y en las Normas de Construcción Sismorresistente de Edificios y de Puentes (NCSE y NCSP)

El empleo de técnicas de construcción novedosas, unido al empleo de simulaciones estructurales durante la fase de diseño de los edificios cada vez con mayor detalle, da lugar a modelos de gran tamaño que pueden alcanzar cientos de miles de grados de libertad.

Un edificio tiene además unas masas y rigideces muy variables, más aún en edificios altos, que pueden albergar desde oficinas hasta centros comerciales, lo que hace que sea un requisito estudiar la respuesta estructural del mismo en las diferentes condiciones.

El método DACMAM promete reducciones de los tiempos de proceso y de los tamaños de ficheros de análisis que resultan de gran interés en modelos de gran tamaño y ante problemas con un número elevado de parametrizaciones. Gracias a su sencillez, se puede implementar de modo relativamente fácil por los ingenieros dedicados al cálculo de estructuras, de modo que tienen control total sobre las parametrizaciones consideradas, que, en el caso descrito comprenderían desde la carga del terremoto (diferentes perfiles de carga), hasta variaciones en la masa y 
rigideces de la estructura, junto con parametrizaciones de equipos específicos de atenuación de vibraciones como son los tunned mass damper (TMD).

La carga a aplicar se trata del terremoto de El Centro del año 1940, en el sur de California, EE.UU (Trifunac y Brune [1970]), ya que los datos del mismo están disponibles libremente en internet (figura 5-5). Este terremoto tuvo una magnitud de 7.1 en la escala Richter, provocando numerosos daños materiales e incluso personales.

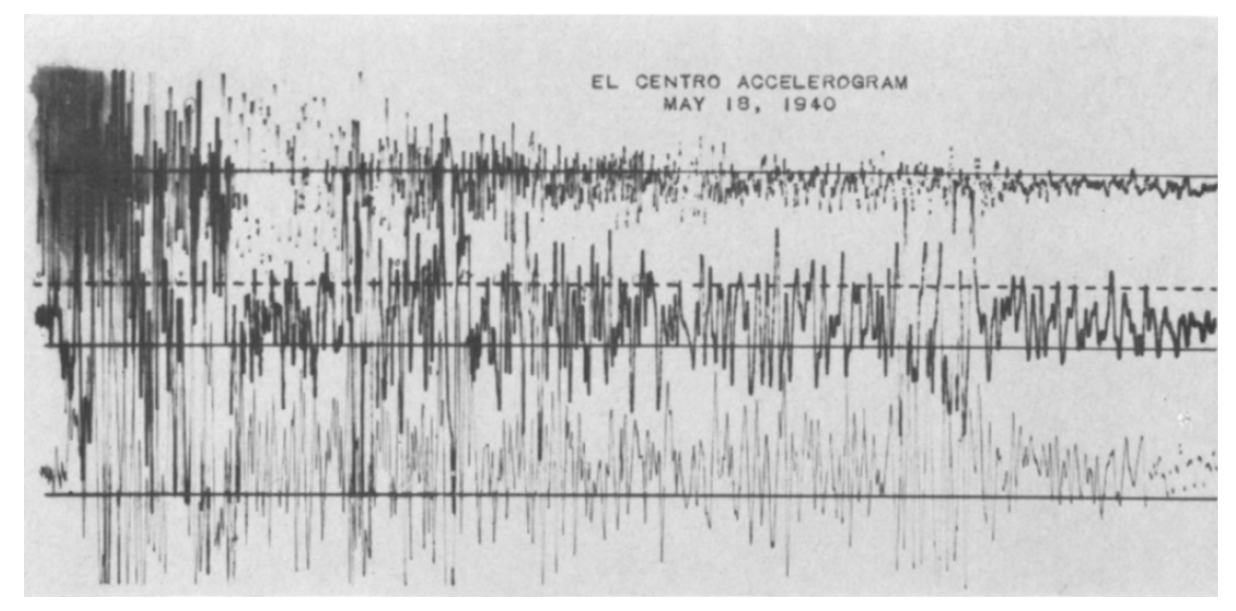

Figura 5-5: Imagen original del acelerómetro que captó la señal del terremoto de El Centro, 1940. Componentes vertical, N-S, y E-O.

Los registros disponibles son registros de aceleraciones en función del tiempo de un sismógrafo muy cercano al epicentro del terremoto. Hay disponibles dos registros, uno correspondiente a la componente norte-sur (N-S), que será el que se emplee en los ejemplos, y otro correspondiente a la componente este-oeste (E-O). Tienen una frecuencia de muestreo de $50 \mathrm{~Hz}$ y un tiempo total de casi $54 \mathrm{~s}$.

Se muestran en las figuras (5-6 y 5-7) dos gráficas correspondientes a la aceleración del terreno en función del tiempo y de la transformada discreta de Fourier (DFT) de la misma. La transformada de Fourier proporciona con todos los datos disponibles del registro del terremoto un $\Delta f_{D F T}=0,012 H z$. Como se comenta más adelante, se refina la densidad de puntos de la DFT añadiendo ceros al final de la señal temporal antes de realizar la transformada con el fin de mejorar la calidad de los resultados. 


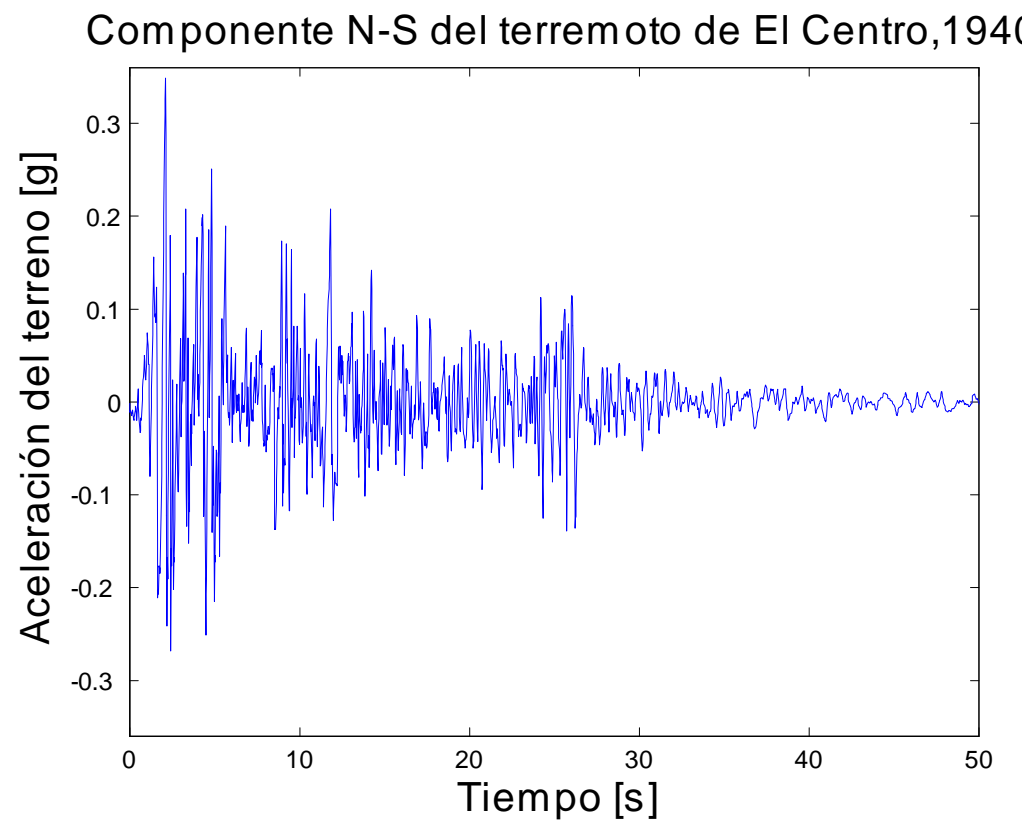

Figura 5-6: Curva de aceleración del terreno, en función del tiempo, para la componente N-S del terremoto de El Centro, 1940.

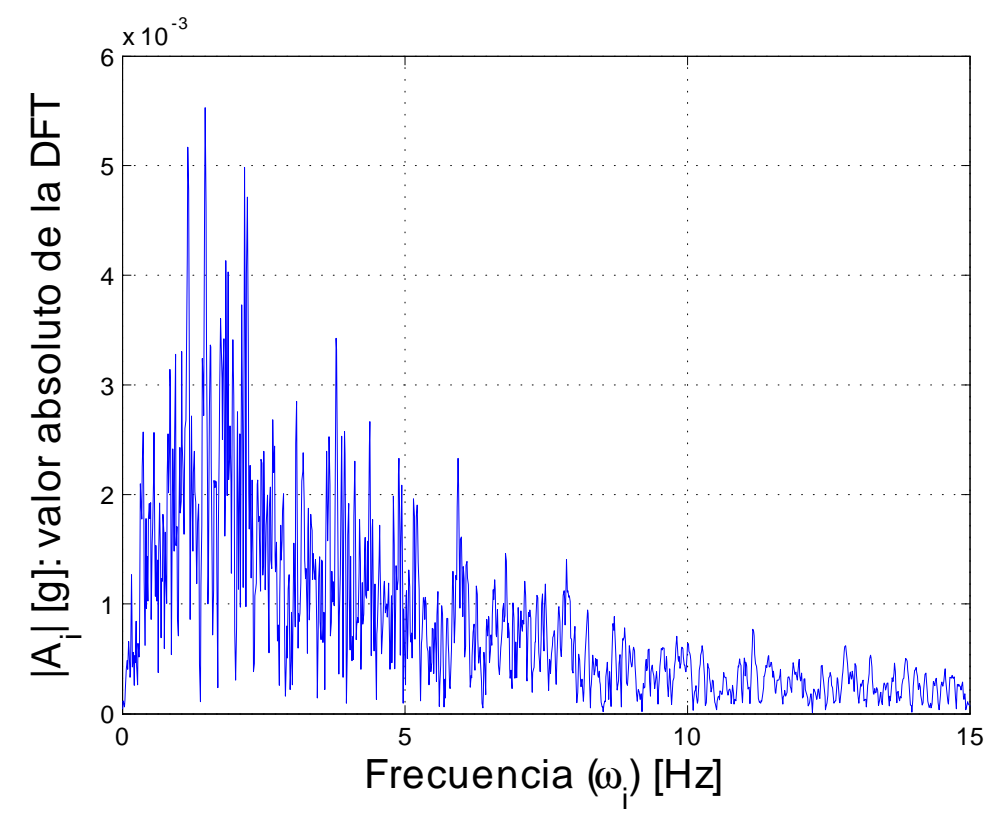

Figura 5-7: Transformada discreta de Fourier para la componente N-S de la aceleración del terreno en el terremoto de El Centro, 1940. 


\section{Capítulo 6}

\section{COMPARACIÓN DE LOS MÉTODOS}

En primer lugar cabe señalar que, para que la comparación de los resultados sea coherente, en el nuevo método se ha empleado truncatura de modos de forma que la dimensión del problema de álgebra compleja tenga la misma dimensión en los métodos que se comparan. En los métodos de reducción por condensación la dimensión del problema es igual a dos veces el número de grados de libertad maestros de los métodos de reducción. En el método DACMAM la dimensión es dos veces el número de modos iniciales retenidos.

\subsection{Tiempos de computación}

Se han realizado los análisis en diversos equipos informáticos para tener en cuenta diversos factores que afectan al tiempo de computación. Por un lado está la velocidad del procesador, que influye en la rapidez para la obtención de los resultados de operaciones matemáticas (la arquitectura también influye en el valor de error de máquina), y por otro lado está la velocidad de acceso a memoria, que influye en el tiempo necesario para ir almacenando la información generada y el acceso a los datos necesarios para realizar las operaciones matemáticas.

Se describen en las tablas (6.1 y 6.2) brevemente las características de los equipos empleados.

La herramienta empleada para la implementación de los métodos es MATLAB [2014]. Hay que tener en cuenta que los tiempos de computación que proporciona emplean el reloj del 


\begin{tabular}{|l|c|c|c|}
\cline { 2 - 4 } \multicolumn{1}{l|}{ Procesador } & Portátil & Estación A & Estación B \\
\hline Año & 2010 & 2013 & 2007 \\
\hline Núcleos & 2 & 8 & 8 \\
\hline$G H z$ & 2.4 & 3.6 & 3 \\
\hline$F S B[M T / s]$ & 667 & 1600 & 1333 \\
\hline Arquitectura & intel core duo (P6) & intel Xeon (Hasswell) & intel Xeon (Core) \\
\hline$n m$ & 65 & 32 & 65 \\
\hline Cache $[M B]$ & $6(\mathrm{~L} 2)$ & 10 (L2 smart) & 8 (L2) \\
\hline
\end{tabular}

Tabla 6.1: Descripción del procesador de los equipos empleados en el análisis.

\begin{tabular}{|l|c|c|c|}
\cline { 2 - 4 } \multicolumn{1}{c|}{ RAM } & $G B$ & Tipo & $M H z$ \\
\hline Portátil (2010) & 4 & DDR3 & 1066 \\
\hline Estación de trabajo A (2013) & 8 & DDR3 & 1600 \\
\hline Estación de trabajo B (2007) & 10 & DDR2 ECC & 667 \\
\hline
\end{tabular}

Tabla 6.2: Descripción de la memoria de acceso aleatorio de los equipos empleados en el análisis.

ordenador, dando lugar a precisiones muy reducidas, dependiente de la máquina y del sistema operativo empleado (en todos los casos se ha empleado Windows 7), ya que este último realiza operaciones o procesos automáticos que consumen capacidad del procesador y de la memoria, y provocan que una misma máquina dé tiempos distintos en diversos momentos. Tras diversas pruebas realizadas se puede considerar que la precisión proporcionada está en el rango 0.01s-0.2s teniendo en cuenta todos los efectos mencionados. Para solventar esta baja precisión, y por los reducidos tiempos de computación para resolver el problema debido a su pequeño tamaño, se han repetido las operaciones un número elevado de veces (del orden de centenas) y a continuación se ha promediado el tiempo empleado.

También se ha procurado en todo caso que las implementaciones sean lo más eficientes posible. Las operaciones que requieren los métodos van desde la resolución de sistemas lineales (no se han invertido matrices en ningún caso, sino que se han resuelto los sistemas lineales correspondientes por medio de factorizaciones) y el producto de matrices y escalares, hasta el cálculo de autovalores y almacenaje de resultados en memoria (esta última se refiere al almacenaje requerido para su uso en memoria de acceso aleatorio y en ningún caso se ha tenido en cuenta el tiempo requerido para almacenar en memoria de acceso directo como son discos duros, etc.).

En pos de la eficiencia, por ejemplo, para la resolución de sistemas lineales empleando fac- 
torizaciones, se han almacenado dichas factorizaciones en memoria para no repetir operaciones cuando era innecesario, se ha minimizado en la programación el uso de bucles, y se ha computado el tiempo requerido tan sólo de las operaciones específicas y necesarias de cada método (e.g. el ensamblaje de matrices, que es común a todos los métodos comparados, no se ha tenido en cuenta en el tiempo de computación). En todo caso, al haberse realizado para esta tesis cada una de las diferentes implementaciones, es de esperar el mismo nivel de eficiencia en todas ellas.

En la colección de tablas que sigue se presentan los resultados para los métodos indicados por filas en el orden que se indica. Los dos primeros corresponden a la resolución del modelo completo sin reducción, en primer lugar para un espacio de los estados que da lugar a matrices no simétricas (formulación estándar), y un segundo en el que el sistema resuelto sí es simétrico (formulación generalizada). El tercer método es el método DACMAM, cuya comparación y validación es el motivo de esta tesis, Ec. (2.16). Los siguientes métodos corresponden, por este orden, al método de condensación estática en el espacio de los desplazamientos, Ec. (3.18), el método de condensación estática en el espacio de los estados I, Ec. (3.32), el método de condensación estática en el espacio de los estados II, Ec. (3.43), el método de condensación dinámica en el espacio de los estados I, Ec. (3.54), el método de condensación dinámica en el espacio de los estados I con una condición inicial distinta, Ec. (3.57), el método de condensación dinámica en el espacio de los estados II, Ec. (3.64), el método de condensación dinámica en el espacio de los estados III, Ec. (3.72), el método de condensación dinámica en el espacio de los estados I híbrido con el espacio III, Ec. (3.74), y por último el método de condensación dinámica en el espacio de los estados II híbrido con el espacio III, Ec. (3.75).

En primer lugar, se han comparado los tiempos de computación para obtener la solución del problema de álgebra compleja, realizando un análisis de respuesta en frecuencia de $0.01 \mathrm{~Hz}$ a $4 \mathrm{~Hz}$ con 327 puntos intermedios, tabla (6.3). En esta primera comparación de tiempos, no hay ninguna reducción de grados de libertad ni truncatura de modos, por lo que la calidad de los resultados es idéntica en todos los casos.

Como primera conclusión, fijándonos en las diferencias de computación entre el método con el modelo completo con un sistema de matrices no simétricas y el método DACMAM, se observan reducciones de tiempos de un $50 \%$ a un $75 \%$. Este valor es una diferencia destacable, más si se tienen en cuenta los siguientes motivos. El orden o tamaño de los sistemas resueltos 


\begin{tabular}{|l|c|c|c|}
\cline { 2 - 3 } \multicolumn{1}{c|}{ tiempos [s] } & Portátil & Estación 2013 & Estación 2007 \\
\hline Espacio de los estados no simétrico, sin reducción & 5.19 & 3.73 & 5.97 \\
\hline SS I, sin reducción & 4.77 & 3.70 & 5.74 \\
\hline Método DACMAM & 1.38 & 1.12 & 3.16 \\
\hline DCSS I, caso A & 4.84 & 3.40 & 4.11 \\
\hline DCSS I, caso B (cond. iniciales diferentes) & 4.73 & 3.44 & 3.94 \\
\hline DCSS II, caso A & 4.87 & 3.33 & 3.83 \\
\hline DCSS III & 4.83 & 3.32 & 3.78 \\
\hline DCSS I, caso C (híbrido, con T del SS III) & 4.78 & 3.38 & 3.97 \\
\hline DCSS II, caso B (híbrido, con T del SS III) & 4.88 & 3.33 & 3.91 \\
\hline SCDS, (resuelto en SS I) & 4.81 & 3.87 & 3.79 \\
\hline SCSS I & 4.82 & 3.48 & 3.82 \\
\hline SCSS II & 4.87 & 3.35 & 3.66 \\
\hline
\end{tabular}

Tabla 6.3: Tiempos de computación de los diferentes métodos sin reducción.

por ambos métodos mediante de álgebra compleja es el mismo, pero hay dos diferencias:

- Mientras en el primer método se evalúa la respuesta de todos los grados de libertad de la estructura (41 gdl), en el segundo sólo se evalúa la respuesta de unos pocos grados de libertad ( $5 \mathrm{gdl}$ ), aquellos en los que queremos supervisar la respuesta, pero sin pérdida de información. A tenor de lo reducido del modelo, es de esperar que para grandes modelos, donde el número de grados de libertad que habitualmente se desea supervisar es varios órdenes de magnitud inferior al número total de grados de libertad del modelo, la reducción en tiempos de computación será mucho mayor. Un ejemplo de ello corresponde a la presentación del método, que es aplicado a un análisis paramétrico sobre un ala de una aeronave no tripulada, (Herrada-Martín [2016]), y donde se muestran ahorros de tiempo con una relación de 1200 a 1 frente a un software comercial de elementos finitos.

- En método completo con un sistema de matrices no simétricas realiza un único cálculo de autovalores, que se emplea en el cambio de coordenadas del espacio físico al espacio modal complejo (de orden 41). En el método DACMAM, se realizan dos cálculos de autovalores, uno para el cambio de variables del espacio real al espacio modal real (de orden 40), y otro adicional para el cambio de variable del espacio modal real al espacio modal complejo, previa incorporación de la información del TMD al modelo transformando sus parámetros físicos al espacio modal real. Al haber empleado la información del modelo completo, este segundo cálculo de autovalores complejos con matrices no simétricas tiene orden 41 tras 
la adición del grado de libertad del TMD.

Este es uno de los aportes que hace el método, y es coherente con el hecho de que habitualmente no es necesario conocer la respuesta de toda la estructura a cargas dinámicas, sino que con determinados puntos de la misma (cuya selección dependen de la experiencia del ingeniero y del conocimiento que se tiene de la estructura) es suficiente para los problemas que se plantean en la gran mayoría de los casos. En el caso del edificio, la última planta es el candidato óptimo para supervisar la variación de las respuestas máximas ante las modificaciones estructurales que se realizan.

Otro de los aspectos a señalar de las comparaciones de los tiempos es que en los equipos más nuevos el método DACMAM proporciona tiempos más reducidos que en el resto de métodos, pero esto apenas sucede en el equipo más antiguo. Todo apunta a que se debe al hecho de que la velocidad de la memoria de acceso aleatorio es menor y que el método DACMAM requiere acceder a los datos almacenados en memoria un número de veces mayor.

En la tabla (6.4) se muestra la comparación de tiempos de computación para los modelos reteniendo 5 grados de libertad (plantas 10, 20, 30, 40 y el gdl correspondiente al TMD). Para que la comparación sea coherente, se ha truncado el número de modos que emplea el método $D A C M A M$ a 5 , de modo que los problemas de álgebra compleja que se resuelven en ambos casos tienen la misma dimensión. Comparando el tiempo para el método DACMAM se observa que hay una reducción del $50 \%$ del tiempo empleado, pero hay que tener en cuenta que la reducción de modos de 40 a 5 afecta negativamente a la calidad de los resultados.

Por último, se muestran en la tabla (6.5) los tiempos de computación correspondientes a una reducción a10 grados de libertad (plantas 5, 10, 15, 20, 25, 30, 34, 38, 40 y el gdl correspondiente al TMD).

Un factor que influye en los tiempos de computación, y del que no se ha hablado todavía, es la tolerancia empleada en los análisis iterativos como condición de convergencia. Esta se ha establecido en $10^{-5}$, y es el límite superior admisible para el error relativo de la parte real y la parte compleja de todos los autovalores del modelo reducido. La selección de este valor no es trivial y se comenta la razón de su selección en el apartado en que se compara la calidad de los resultados. También se ha limitado el número máximo de iteraciones como es habitual, siendo el valor seleccionado 50. Este valor se alcanza en los métodos iterativos cuando se toman 10 


\begin{tabular}{|l|c|c|c|}
\cline { 2 - 3 } \multicolumn{1}{c|}{ tiempos [s] } & Portátil & Estación 2013 & Estación 2007 \\
\hline Espacio de los estados no simétrico, sin reducción & 4.78 & 3.78 & 5.83 \\
\hline SS I, sin reducción & 4.69 & 3.72 & 5.73 \\
\hline Método DACMAM & 0.95 & 0.48 & 0.56 \\
\hline DCSS I, caso A & 0.73 & 0.55 & 1.18 \\
\hline DCSS I, caso B (cond. iniciales diferentes) & 0.73 & 0.54 & 1.46 \\
\hline DCSS II, caso A & 0.77 & 0.55 & 1.77 \\
\hline DCSS III & 0.71 & 0.50 & 1.13 \\
\hline DCSS I, caso C (híbrido, con T del SS III) & 0.66 & 0.51 & 0.80 \\
\hline DCSS II, caso B (híbrido, con T del SS III) & 0.80 & 0.50 & 1.27 \\
\hline SCDS, (resuelto en SS I) & 0.55 & 0.43 & 0.45 \\
\hline SCSS I & 0.55 & 0.42 & 0.45 \\
\hline SCSS II & 0.55 & 0.42 & 0.45 \\
\hline
\end{tabular}

Tabla 6.4: Tiempos de computación de los diferentes métodos con reducción a 5 grados de libertad.

grados de libertad maestros, que de acuerdo a la tabla (6.5), dan tiempos de proceso superiores a los del método DACMAM para resultados de una calidad similar o inferior como se verá en el siguiente apartado.

Aunque el empleo de diferentes computadores también influye en el número de iteraciones necesarias para los métodos iterativos y en sus resultados, la influencia es tan pequeña que no se tendrá en cuenta en la valoración de la calidad de los resultados ofrecidos por los diferentes métodos que se exponen en la tesis. Aunque influye en los tiempos de computación por depender éstos, para los métodos iterativos, de los resultados obtenidos, también se puede considerar que la influencia es pequeña y en todo caso menor que la del resto de factores que sí se han tenido en cuenta.

La comparación de tiempos en el caso de tener en cuenta parametrizaciones se abordará más adelante en cada caso de parametrización concreto. 


\begin{tabular}{|l|c|c|c|}
\cline { 2 - 4 } \multicolumn{1}{c|}{ tiempos [s] } & Portátil & Estación 2013 & Estación 2007 \\
\hline Espacio de los estados no simétrico, sin reducción & 4.83 & 3.90 & 5.89 \\
\hline SS I, sin reducción & 4.72 & 3.82 & 5.70 \\
\hline Método DACMAM & 1.72 & 0.96 & 1.14 \\
\hline DCSS I, caso A & 1.26 & 1.07 & 1.63 \\
\hline DCSS I, caso B (cond. iniciales diferentes) & 1.26 & 1.08 & 1.75 \\
\hline DCSS II, caso A & 1.32 & 1.15 & 2.03 \\
\hline DCSS III & 1.31 & 1.13 & 1.93 \\
\hline DCSS I, caso C (híbrido, con T del SS III) & 1.33 & 1.14 & 1.86 \\
\hline DCSS II, caso B (híbrido, con T del SS III) & 1.32 & 1.13 & 1.76 \\
\hline SCDS, (resuelto en SS I) & 1.07 & 0.85 & 0.90 \\
\hline SCSS I & 1.08 & 0.84 & 0.90 \\
\hline SCSS II & 1.10 & 0.91 & 0.90 \\
\hline
\end{tabular}

Tabla 6.5: Tiempos de computación de los diferentes métodos con reducción a 10 grados de libertad.

\subsection{Comparación de autovalores, autovectores y factor de amor- tiguamiento crítico}

Hay diferentes métodos disponibles para la comparación de los autovectores (Allemang y Brown [1982]; Montáns y Muñoz [2013]; Chopra [1995]; Qu [2004]). Al disponer de un ejemplo de tamaño reducido, se puede contar con unos autovectores de referencia del modelo completo, por lo que por su sencillez y por dar como resultado un único escalar por modo, se emplea la siguiente ecuación "Correlated Coefficient For Modal Vector" CCFMV (básicamente consiste en los elementos de la diagonal del Modal Assurance Criterion, MAC):

$$
C C F M V\left(\boldsymbol{\psi}_{i}^{\text {exacto }}, \boldsymbol{\psi}_{i}^{\text {red }}\right)=\frac{\left(\boldsymbol{\psi}_{i}^{\text {exacto }}\right)^{T} \cdot \mathbf{W} \cdot \boldsymbol{\psi}_{i}^{\text {red }}}{\left[\left(\left(\boldsymbol{\psi}_{i}^{\text {exacto }}\right)^{T} \cdot \mathbf{W} \cdot \boldsymbol{\psi}_{i}^{\text {exacto }}\right)\left(\left(\boldsymbol{\psi}_{i}^{\text {red }}\right)^{T} \cdot \mathbf{W} \cdot \boldsymbol{\psi}_{i}^{\text {red }}\right)\right]^{1 / 2}}
$$

Se tomará como autovector de referencia aquel del sistema de ecuaciones completo correspondiente al modo que se compara, pero tomando sólo los grados de libertad que se hayan retenido en el sistema reducido y descartando el resto. De este modo se obtiene un coeficiente por modo. En cuanto a la matriz de peso que se añade, W, se toma la matriz identidad (se podría tomar también la matriz de masas del sistema o la matriz de rigidez).

Este coeficiente se convierte en error por medio de la siguiente ecuación (al trabajar con 
modos complejos, el resultado de CCFMV es en general un número complejo):

$$
\text { Error }_{M O D O i}=\left|C C F M V_{i}-1\right| \cdot 100
$$

Para implementar este cálculo de error en el caso del método DACMAM, al contar éste con dos cambios de espacios, el coeficiente de correlación queda del siguiente modo (para la matriz de peso valdría cualquiera de las dos matrices del sistema en el espacio modal real):

$$
C C F M V\left(\boldsymbol{\psi}_{i}^{\text {exacto }}, \boldsymbol{\psi}_{i}^{D}\right)=\frac{\left(\boldsymbol{\psi}_{i}^{\text {exacto }}\right)^{T} \cdot \mathbf{W} \cdot\left(\tilde{\boldsymbol{\Phi}} \boldsymbol{\psi}_{i}^{D}\right)}{\left[\left(\left(\boldsymbol{\psi}_{i}^{\text {exacto }}\right)^{T} \cdot \mathbf{W} \cdot \boldsymbol{\psi}_{i}^{\text {exacto }}\right)\left(\left(\tilde{\boldsymbol{\Phi}} \boldsymbol{\psi}_{i}^{D}\right)^{T} \cdot \mathbf{W} \cdot\left(\tilde{\boldsymbol{\Phi}} \boldsymbol{\psi}_{i}^{D}\right)\right)\right]^{1 / 2}}
$$

Al ser $\boldsymbol{\psi}_{i}^{D}=\boldsymbol{\psi}_{i}^{D A C M A M}$ el autovector complejo del modo $i$ obtenido del nuevo problema de autovalores en el espacio modal real, la matriz de autovectores en el espacio físico, $\boldsymbol{\Phi}$, se debe ampliar al espacio de los estados, $\tilde{\boldsymbol{\Phi}}$, del siguiente modo:

$$
\tilde{\boldsymbol{\Phi}}=\left[\begin{array}{ll}
\Phi & 0 \\
0 & \Phi
\end{array}\right]
$$

$\mathrm{Al}$ crear la matriz de autovectores ampliada, $\tilde{\boldsymbol{\Phi}}$, se debe tener especial cuidado, añadiendo los autovectores correspondientes a los nuevos grados de libertad como se indica en la descripción del método, Ec. (2.23).

Se muestran a continuación tablas con las comparaciones de resultados modales del problema del edificio con el TMD, para varios tipos de reducciones:

- En primer lugar se presentan los resultados de los primeros 6 modos para los métodos aplicados reteniendo todos los grados de libertad, a modo de verificación de que están bien implementados. La tabla (6.6) muestra los valores obtenidos de los autovalores junto al número de iteraciones requerido para converger (se recuerda que el número máximo de iteraciones se ha limitado a 50), y a continuación se presentan en la tabla (6.7) los errores relativos de los mismos tomando como referencia uno de los métodos sin reducción. Posteriormente, en las tablas (6.8 y 6.9), se muestran del mismo modo los factores de amortiguamiento crítico y por último los errores correspondientes a la correlación de los 
autovectores, obtenidos de acuerdo al método indicado al inicio del apartado. En la tabla del error de los autovectores, tabla (6.10), el autovector de referencia es el correspondiente al método sin reducción en el espacio de los estados no simétrico, la primera fila y que se muestra en la tabla por motivos de verificación.

- En segundo término se muestran los resultados con una reducción del problema a 5 grados de libertad: las plantas 10, 20, 30, 40 y el grado de libertad correspondiente al TMD. Como ya se ha comentado, para realizar una comparación coherente, los resultados mostrados del método DACMAM corresponden a una truncatura modal reteniendo los 5 primeros modos. Los datos se organizan igual que para el caso sin reducción en las tablas (6.11, $6.12,6.13,6.14$ y 6.15$)$.

- Por último se muestran los resultados correspondientes a una reducción del problema a 10 grados de libertad, los mencionados anteriormente y las plantas adicionales 5, 15, 25, 34 y 38. Los datos se presentan en las tablas $(6.16,6.17,6.18,6.19,6.20,6.21,6.22,6.23$, 6.24 y 6.25$)$.

De los resultados mostrados se puede extraer que el error de máquina es del orden de $10^{-14}$. 


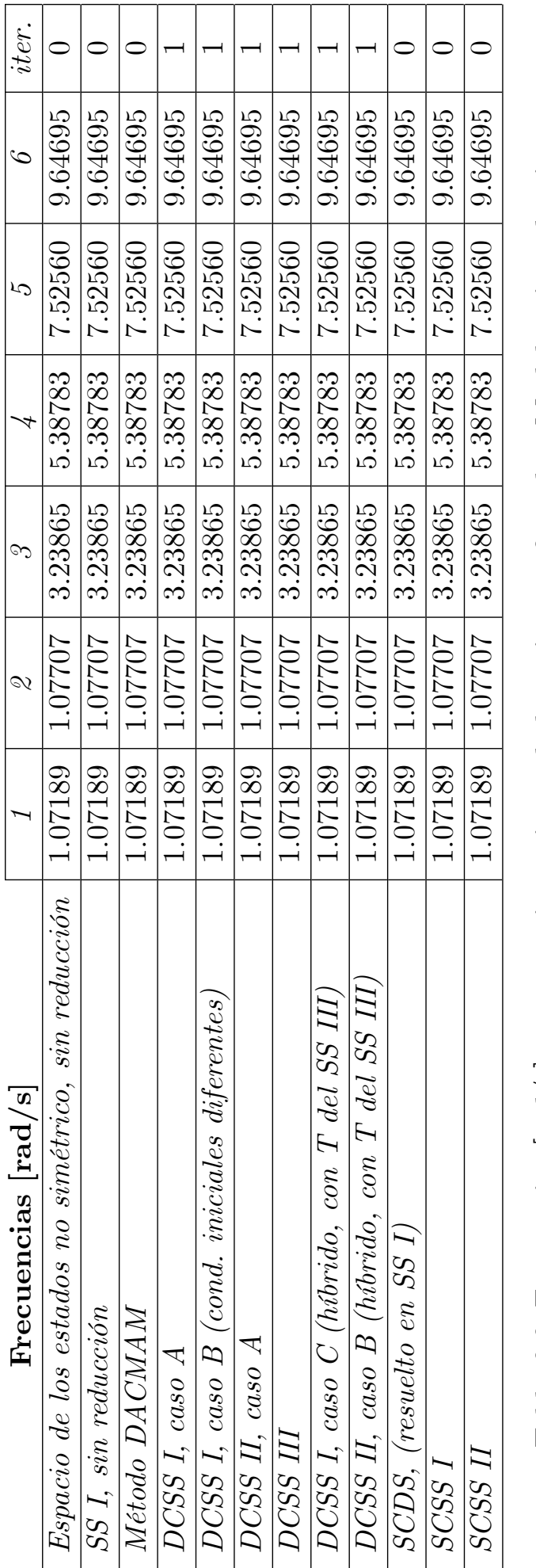

\begin{tabular}{|c|c|c|c|c|c|c|c|c|c|c|c|}
\hline 0 & 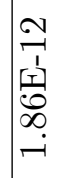 & 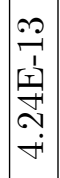 & $\mid \begin{array}{c}\mathcal{N} \\
-1 \\
-1 \\
\\
\infty \\
\infty \\
-1\end{array}$ & $\begin{array}{c}0 \\
-1 \\
-1 \\
-1 \\
01 \\
0 \\
-i \\
-i\end{array}$ & 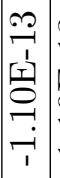 & 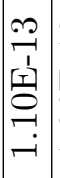 & $\begin{array}{c}N \\
-1 \\
-1 \\
-1 \\
01 \\
\infty \\
-i \\
-i\end{array}$ & 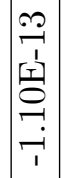 & $\begin{array}{c}N \\
7 \\
1 \\
-1 \\
01 \\
\infty \\
-1 \\
-1\end{array}$ & 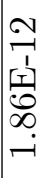 & 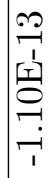 \\
\hline هم & 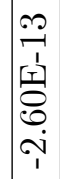 & 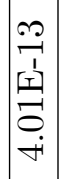 & 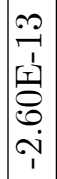 & $\begin{array}{c}0 \\
0 \\
1 \\
-1 \\
0 \\
0 \\
0 \\
i \\
1\end{array}$ & 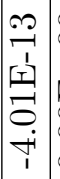 & $\begin{array}{c}8 \\
0 \\
+ \\
\text { 19 } \\
8 \\
8 \\
0\end{array}$ & $\begin{array}{c}\infty \\
-1 \\
1 \\
-1 \\
0 \\
0 \\
ن \\
i \\
1\end{array}$ & 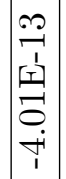 & 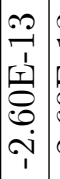 & 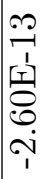 & 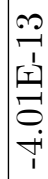 \\
\hline & 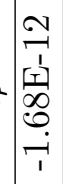 & 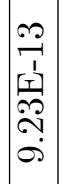 & $\left|\begin{array}{c}\mathcal{N} \\
-1 \\
-1 \\
0 \\
0 \\
0 \\
-1 \\
1\end{array}\right|$ & $\left|\begin{array}{c}0 \\
7 \\
r 1 \\
-1 \\
\infty \\
0 \\
0 \\
-1 \\
1\end{array}\right|$ & 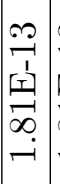 & 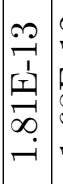 & 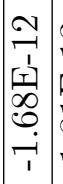 & 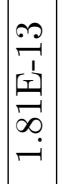 & 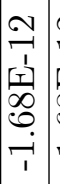 & 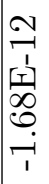 & 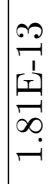 \\
\hline ob & 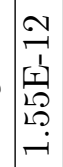 & 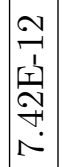 & 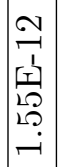 & 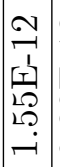 & 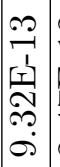 & 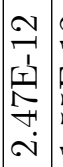 & 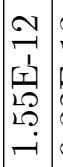 & 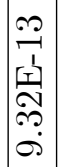 & $\begin{array}{c}N \\
7 \\
-1 \\
12 \\
20 \\
20 \\
-1\end{array}$ & 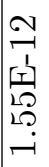 & 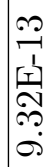 \\
\hline Su & 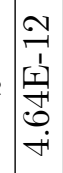 & 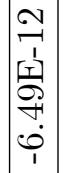 & 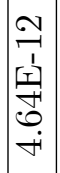 & 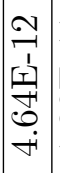 & 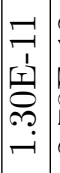 & $\begin{array}{c}\mathcal{N} \\
-1 \\
-1 \\
\infty \\
D \\
N \\
v i\end{array}$ & 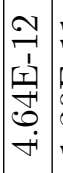 & 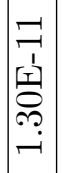 & 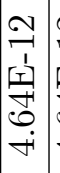 & 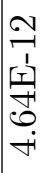 & 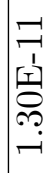 \\
\hline-1 & 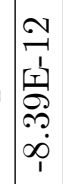 & 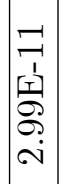 & 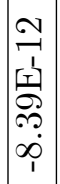 & 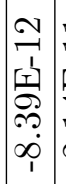 & 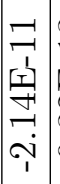 & 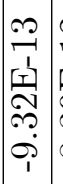 & 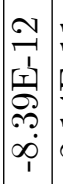 & 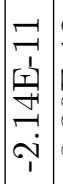 & 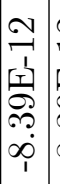 & 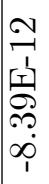 & 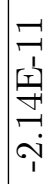 \\
\hline 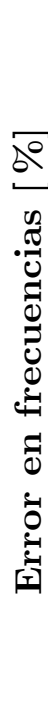 & 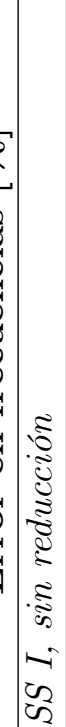 & 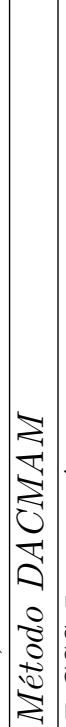 & 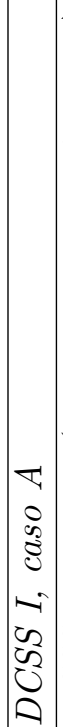 & 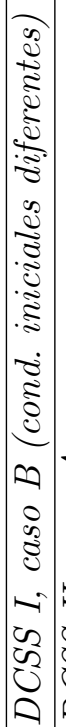 & $\begin{array}{c}7 \\
0 \\
0 \\
0 \\
0 \\
-1 \\
-1 \\
\delta 2 \\
0 \\
0 \\
0\end{array}$ & 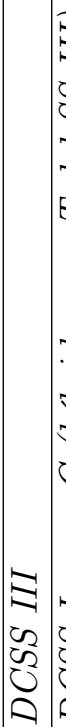 & 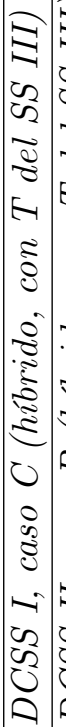 & 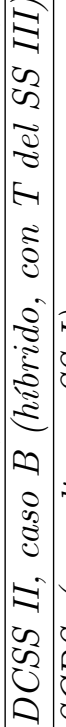 & 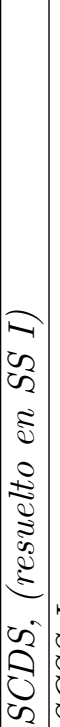 & 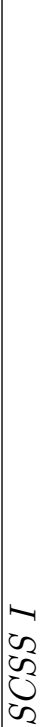 & $\begin{array}{l}\approx \\
\sigma \\
\sigma \\
\sigma \\
\sigma\end{array}$ \\
\hline
\end{tabular}

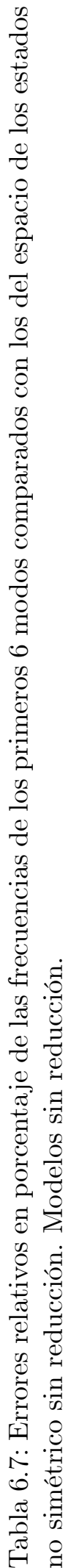




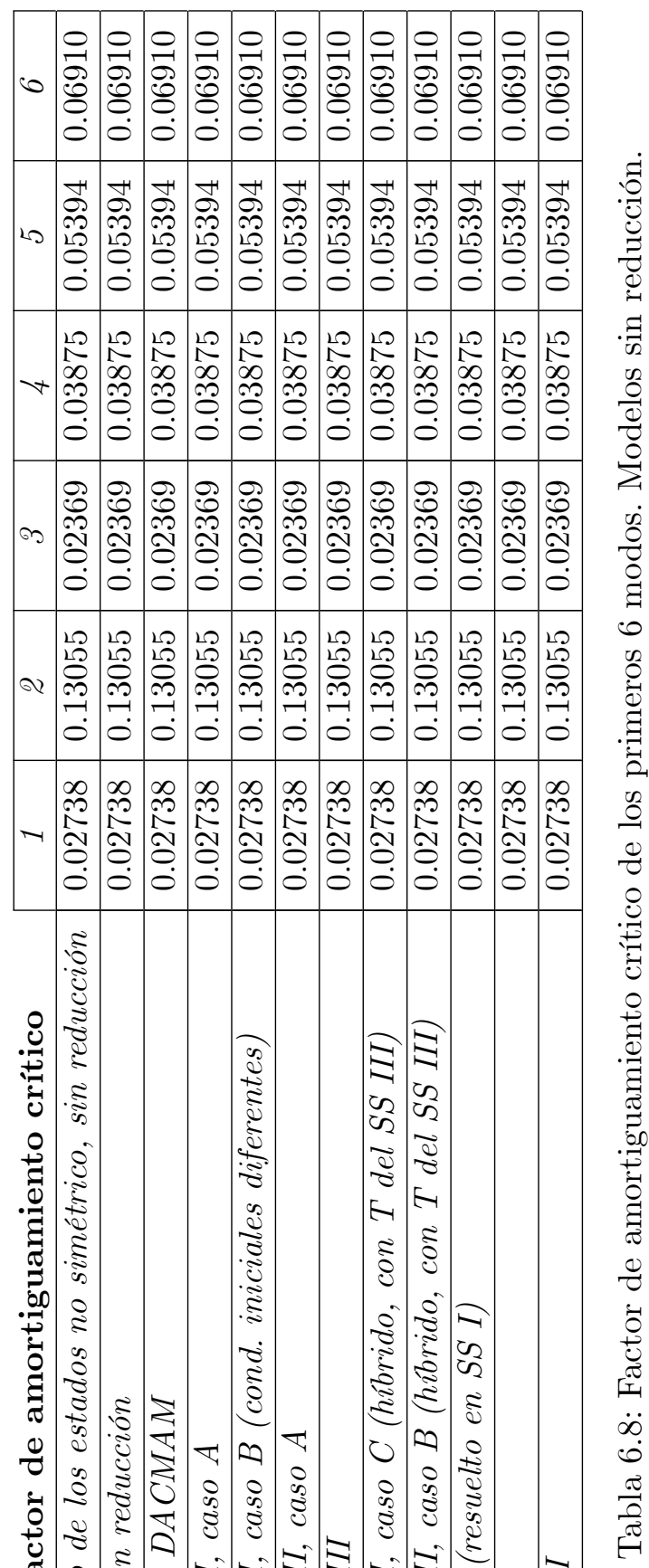

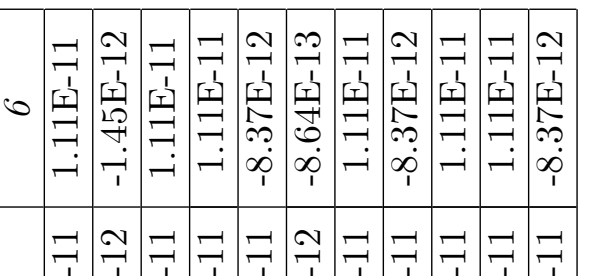

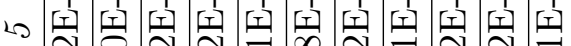

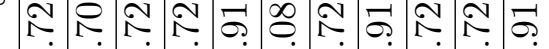

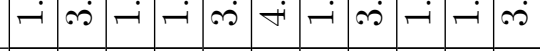

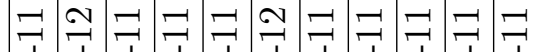

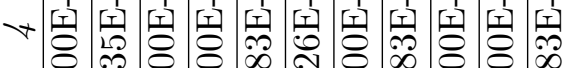

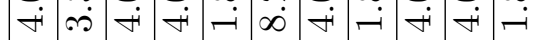

$\exists \exists \exists \exists ㅋ \exists \exists \Xi \exists$

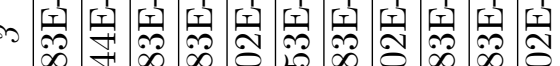

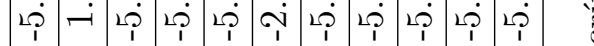

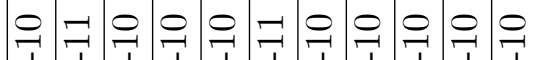

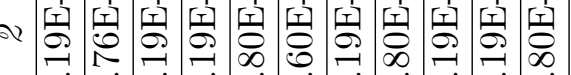

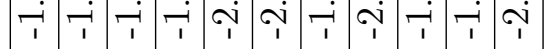

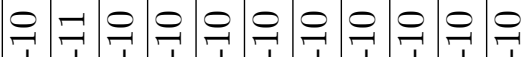

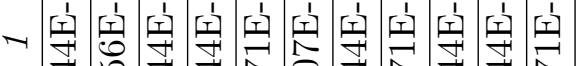

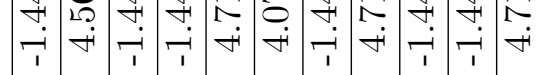

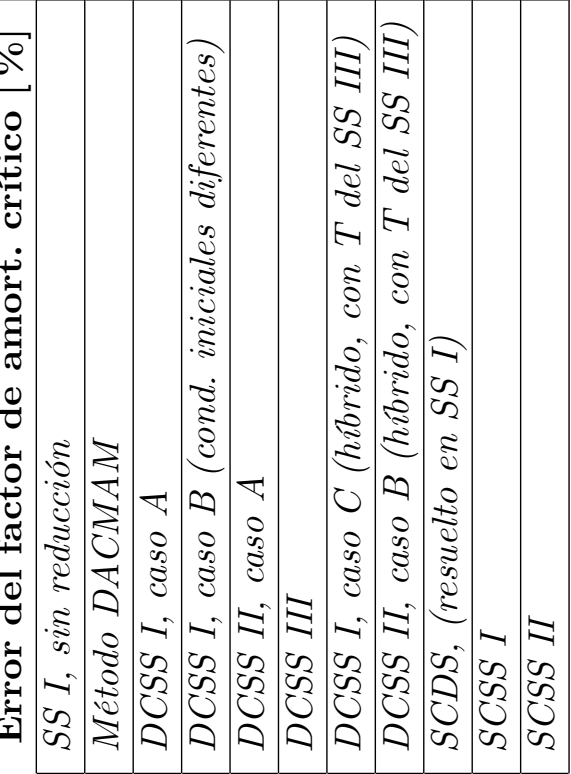

$\stackrel{\infty}{0}$

F

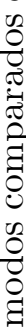

:

$\stackrel{0}{\circ}$

कृ

.8

융

苗

远

द्व

웡

?ै

产

\&

ช)

. $\frac{.}{\pi}$

迅

ํ. 요

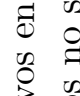

.

एँ

क्ष है

는

$\ddot{\circ} \cdot$

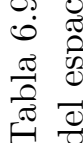




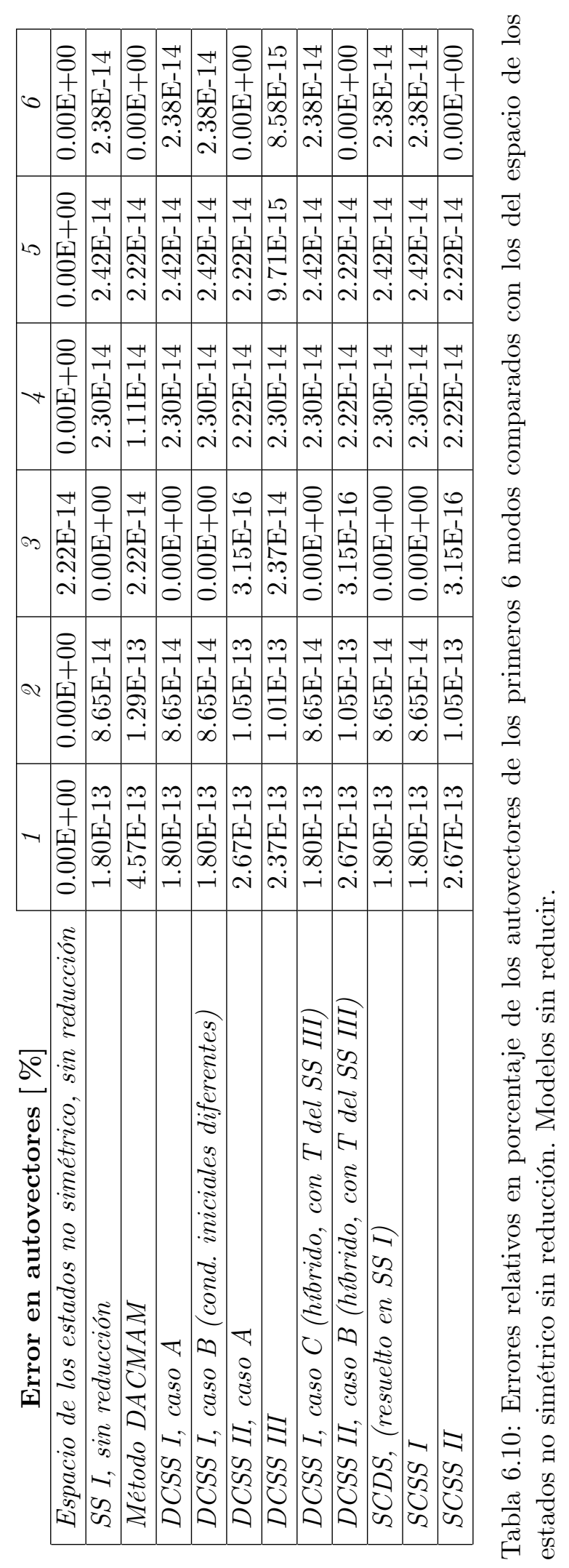




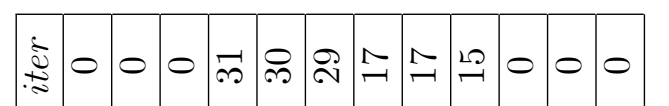

율

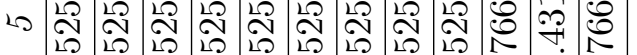

N

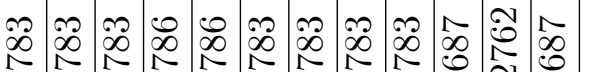

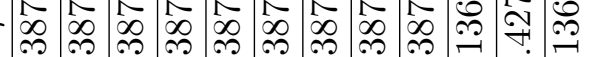

ف

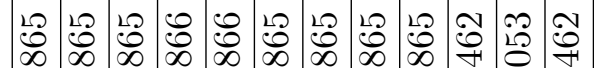

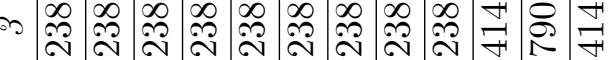
ल

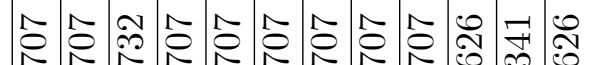

Q

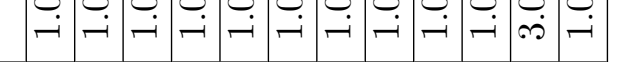

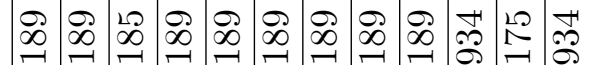

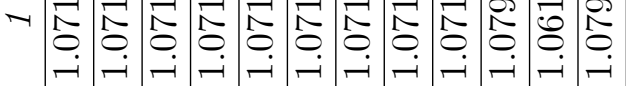

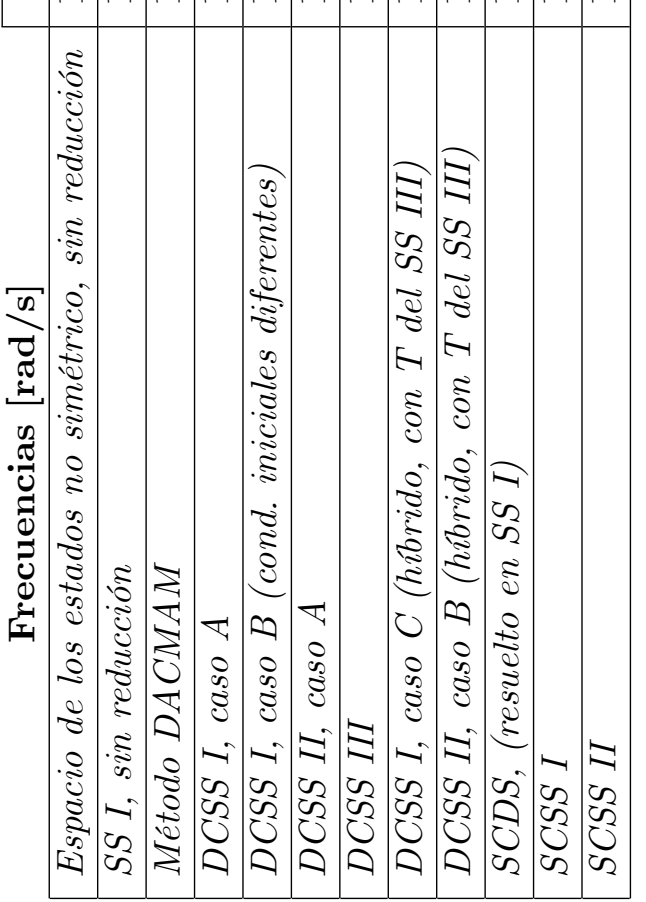

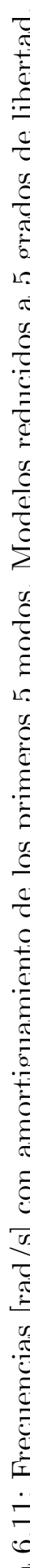

$\frac{\frac{\pi}{0}}{\frac{\pi}{4}}$
.9

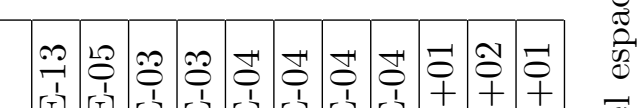

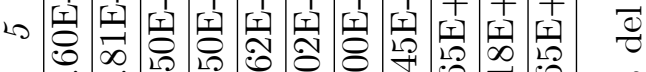

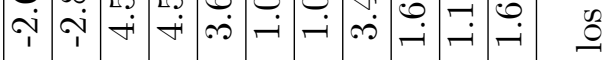

ㄱำ

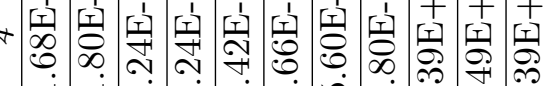

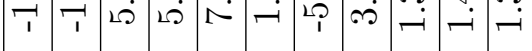

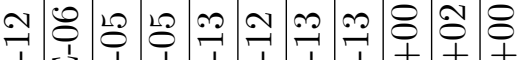

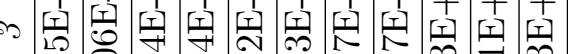

总

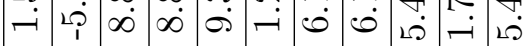

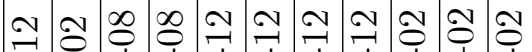

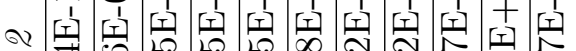

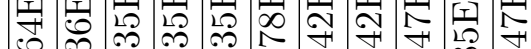

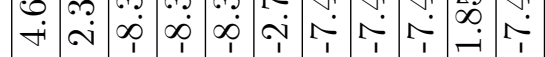

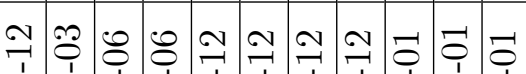

- 寊

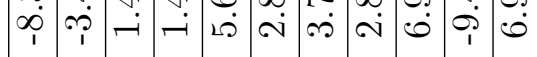

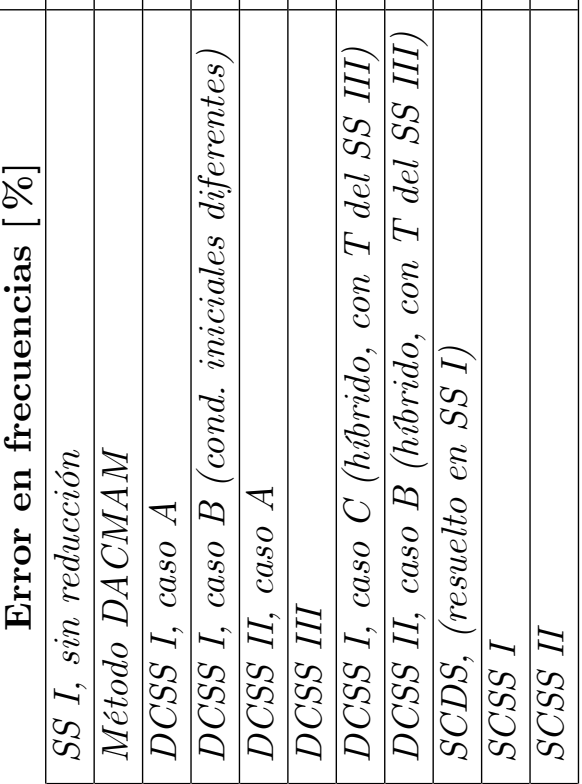

n

离

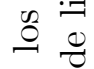

ชै क

?ี

ठै

ن

$\because \frac{0}{0}$

赵

ช

疍

륭

8

훙

त)

?

$\frac{\pi}{0} \cdot \exists$

过

$\ddot{\sim}$

0

竞 


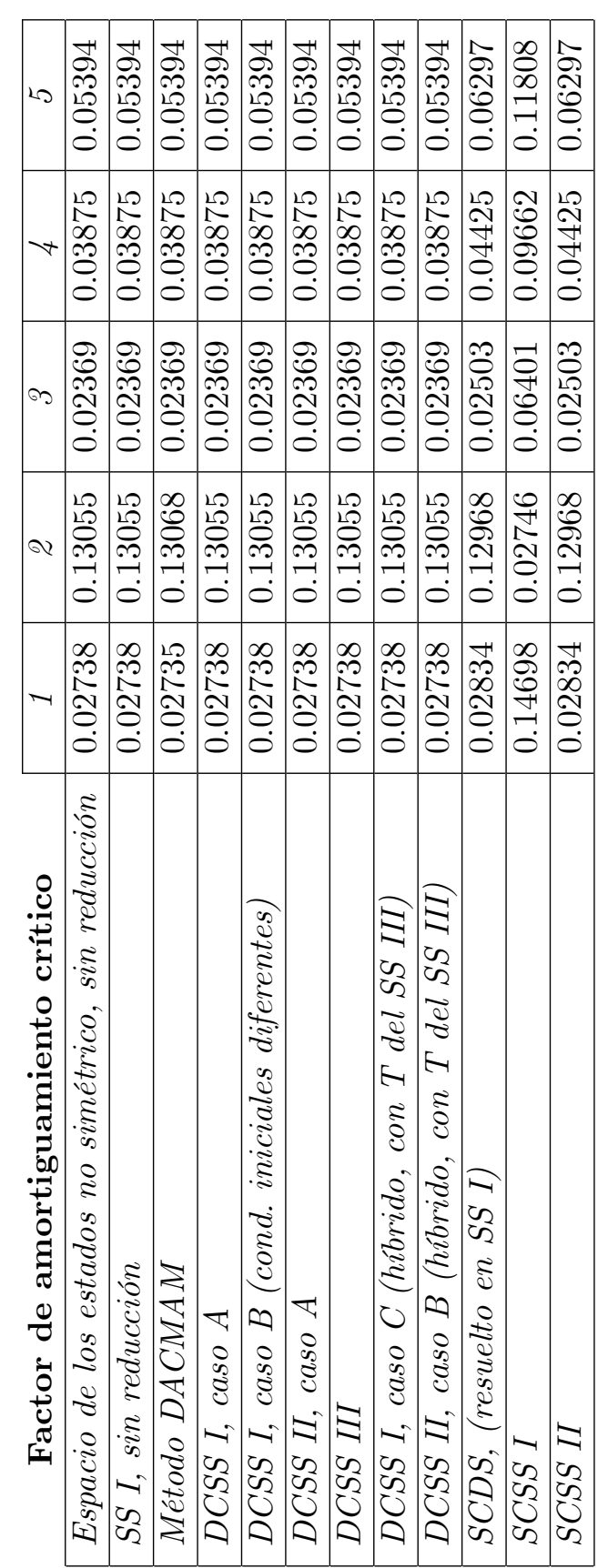




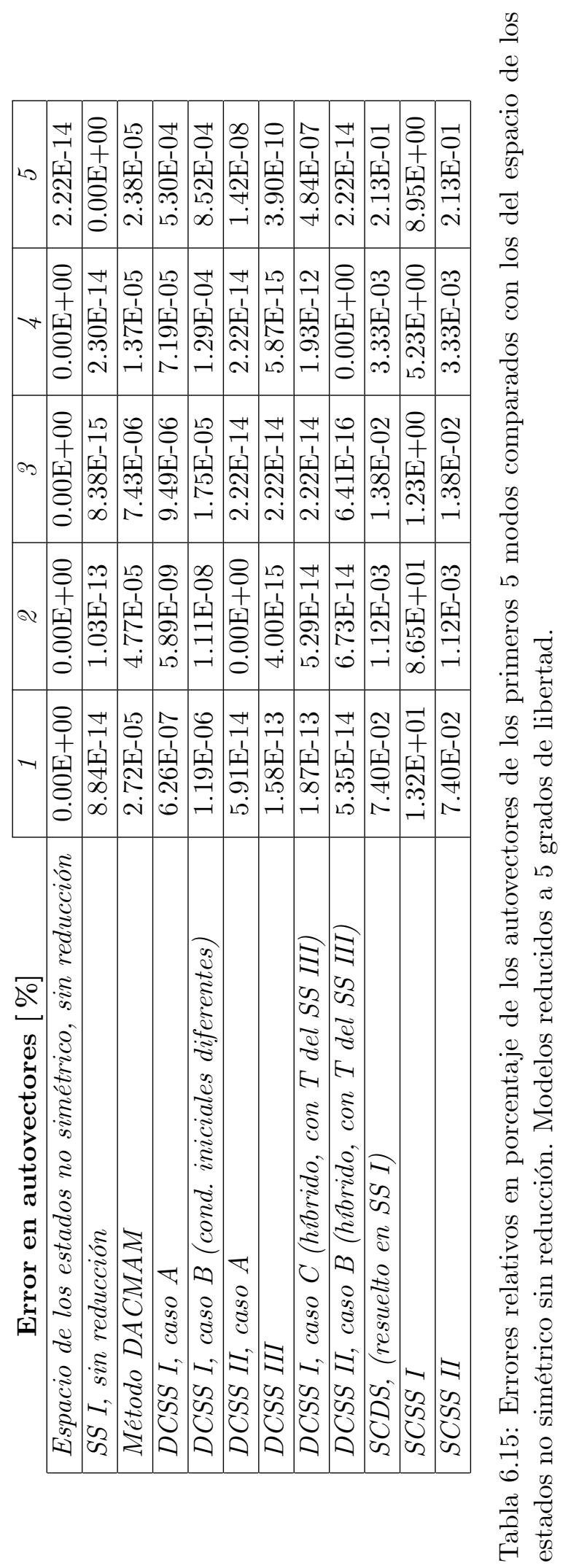




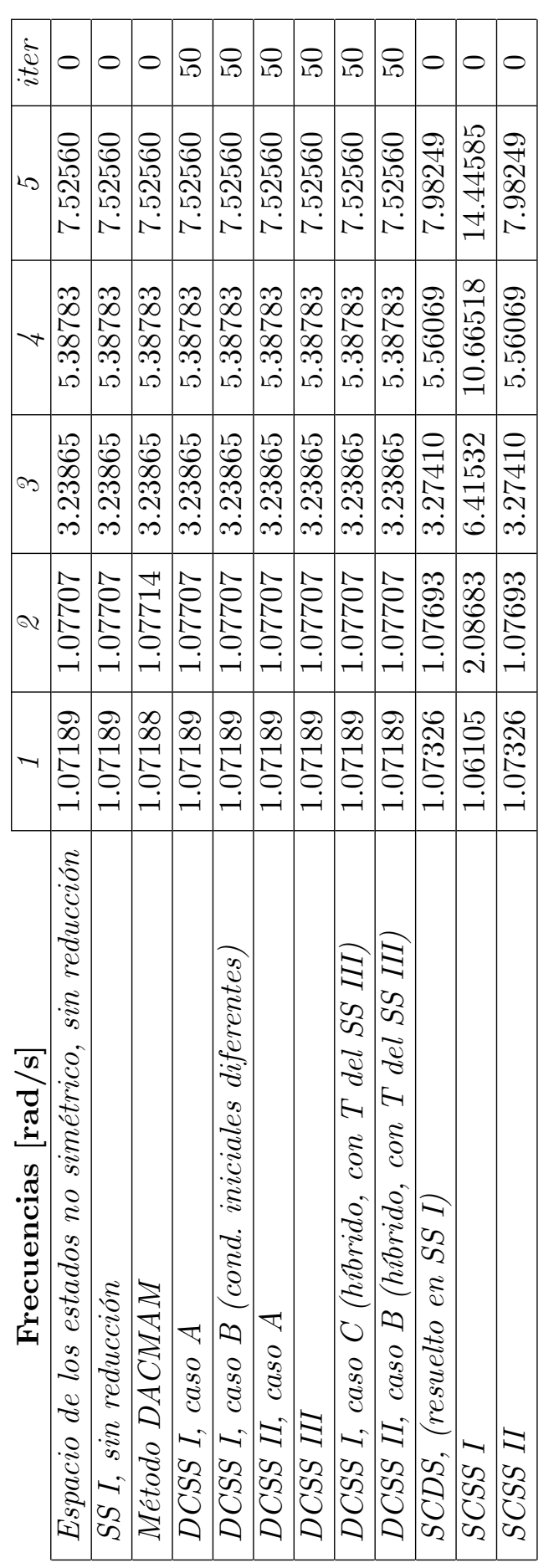

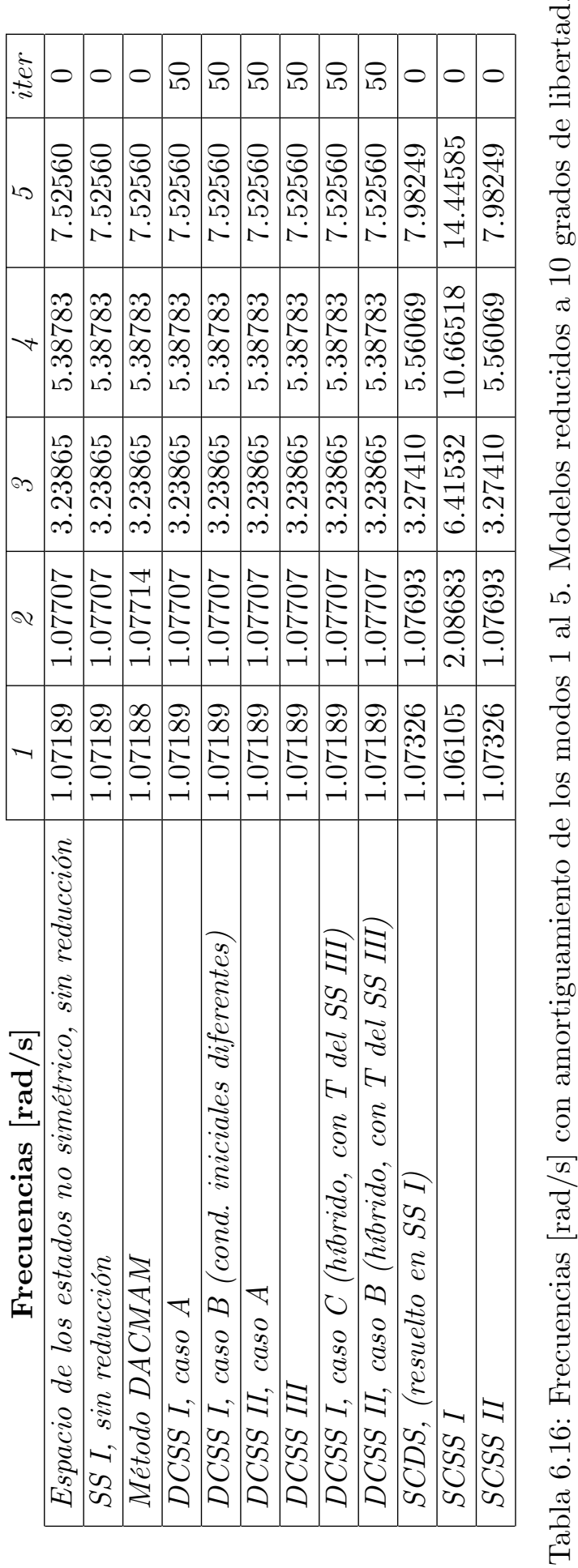

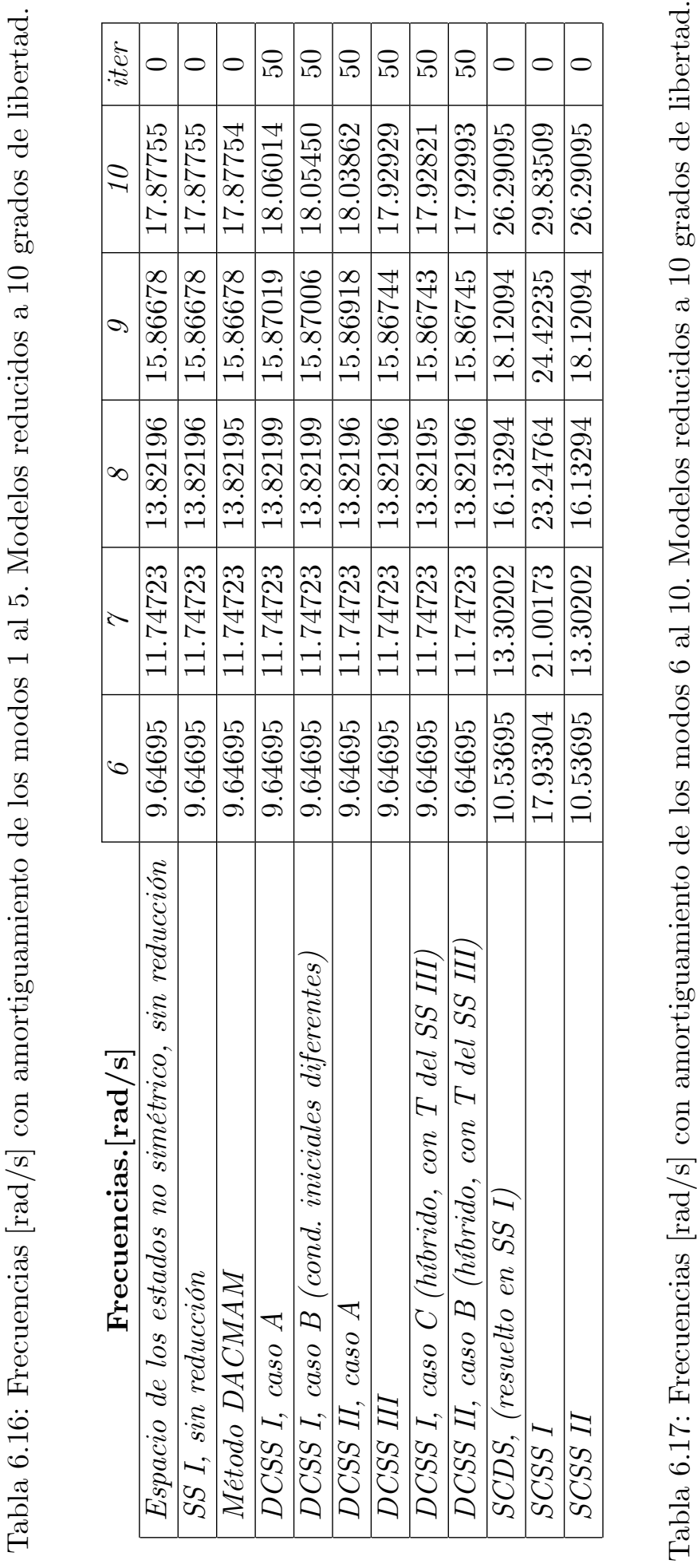




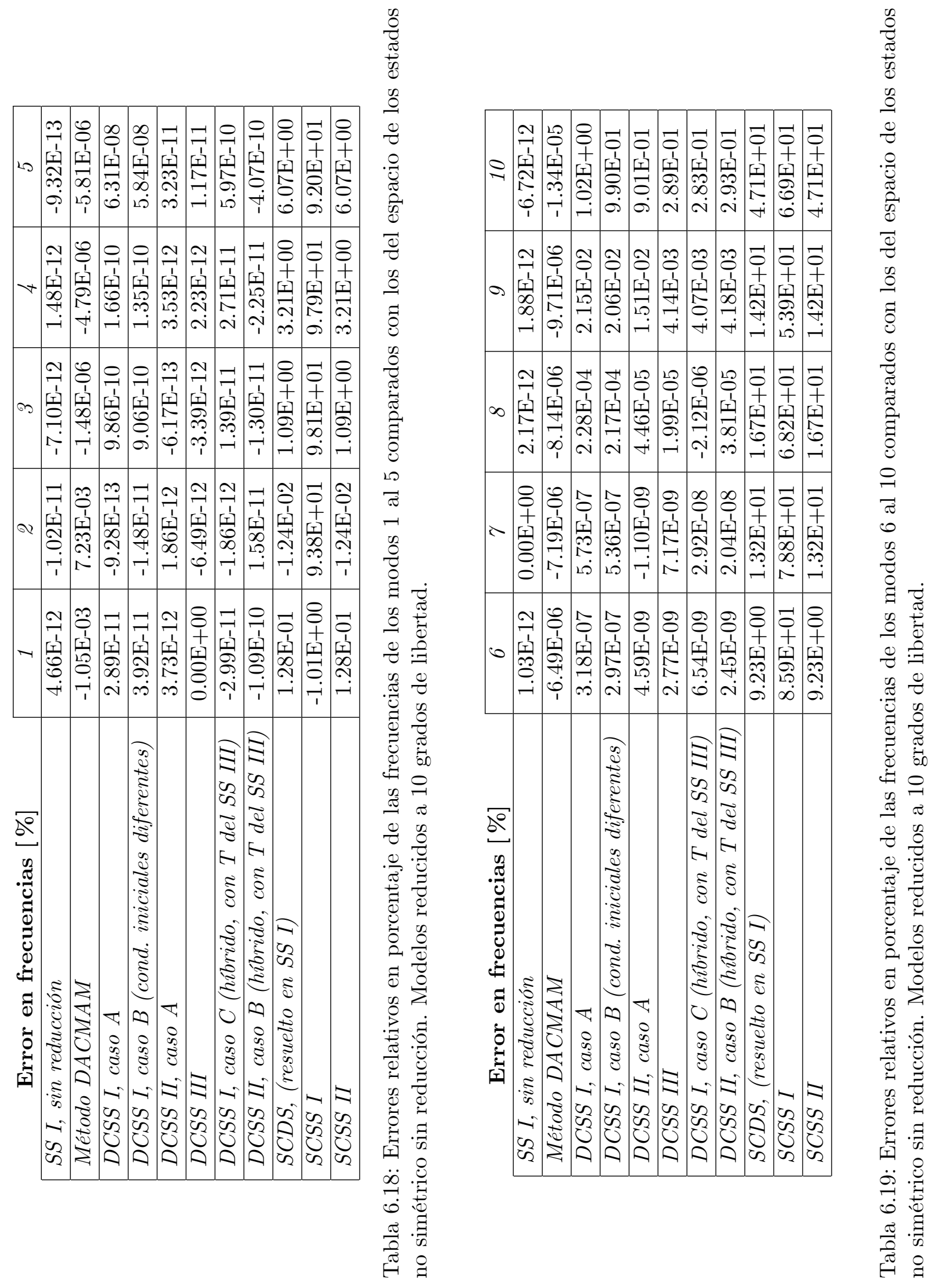




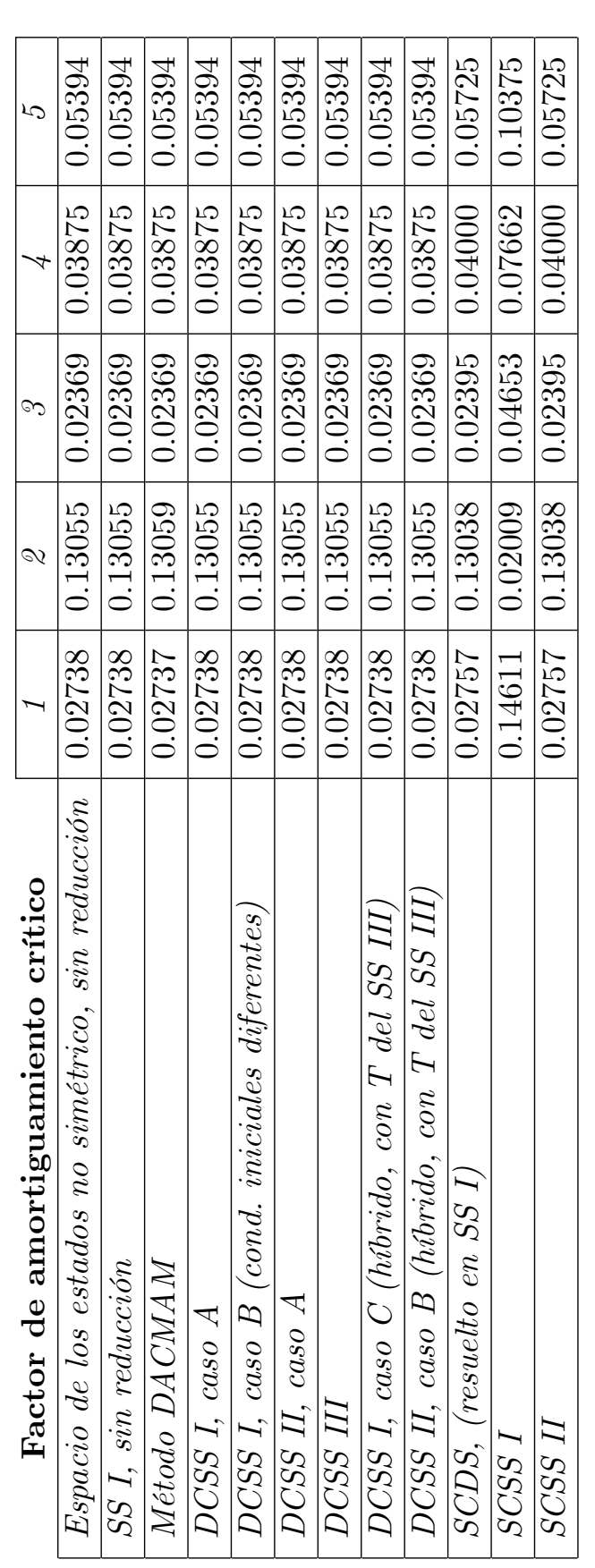

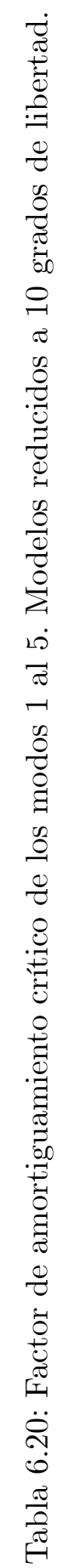

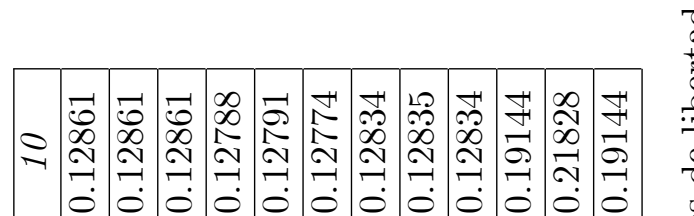

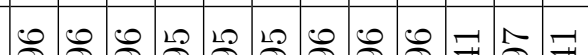
\&

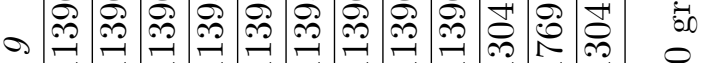

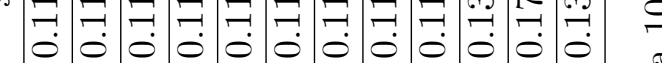

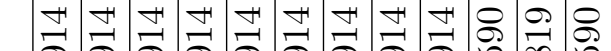

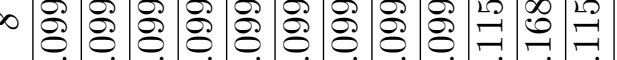

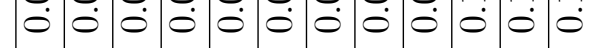
$\infty \infty \infty \infty \infty \infty \infty \infty \infty \cdots$

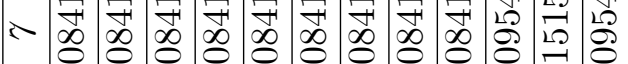
$\begin{array}{lllllllllllll}0 & 0 & 0 & 0 & 0 & 0 & 0 & 0 & 0 & 0 & 0 & 0 \\ 0\end{array}$

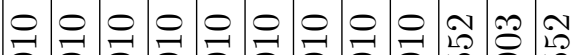

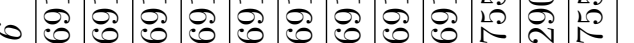

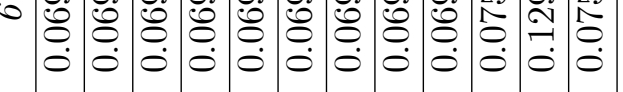

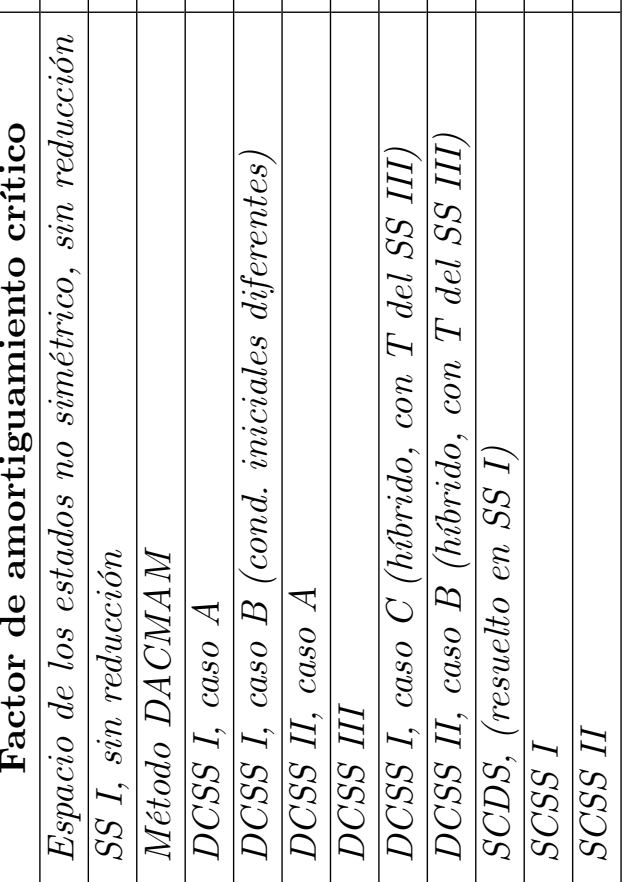

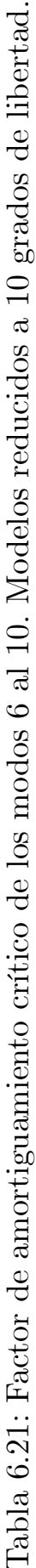




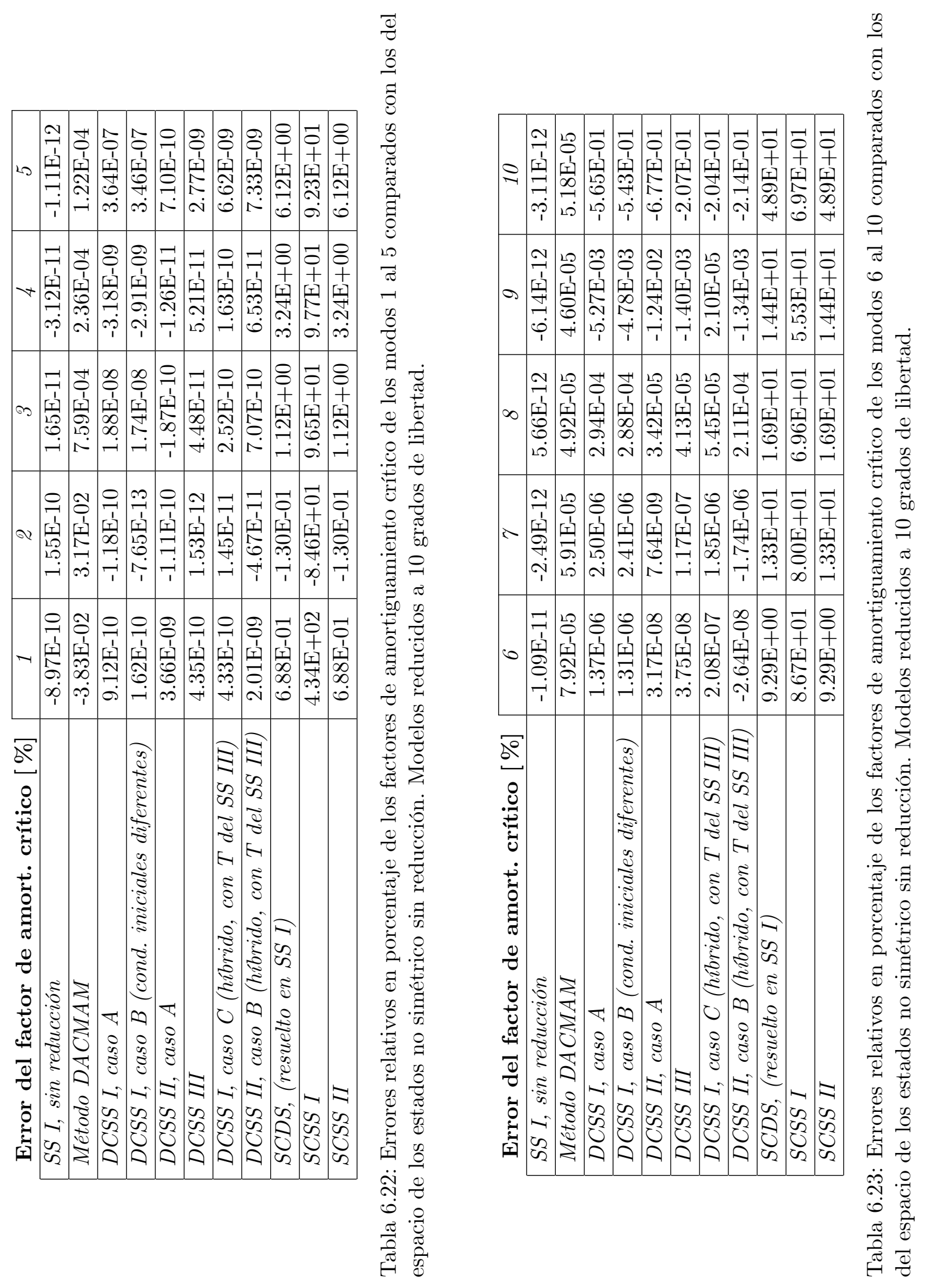



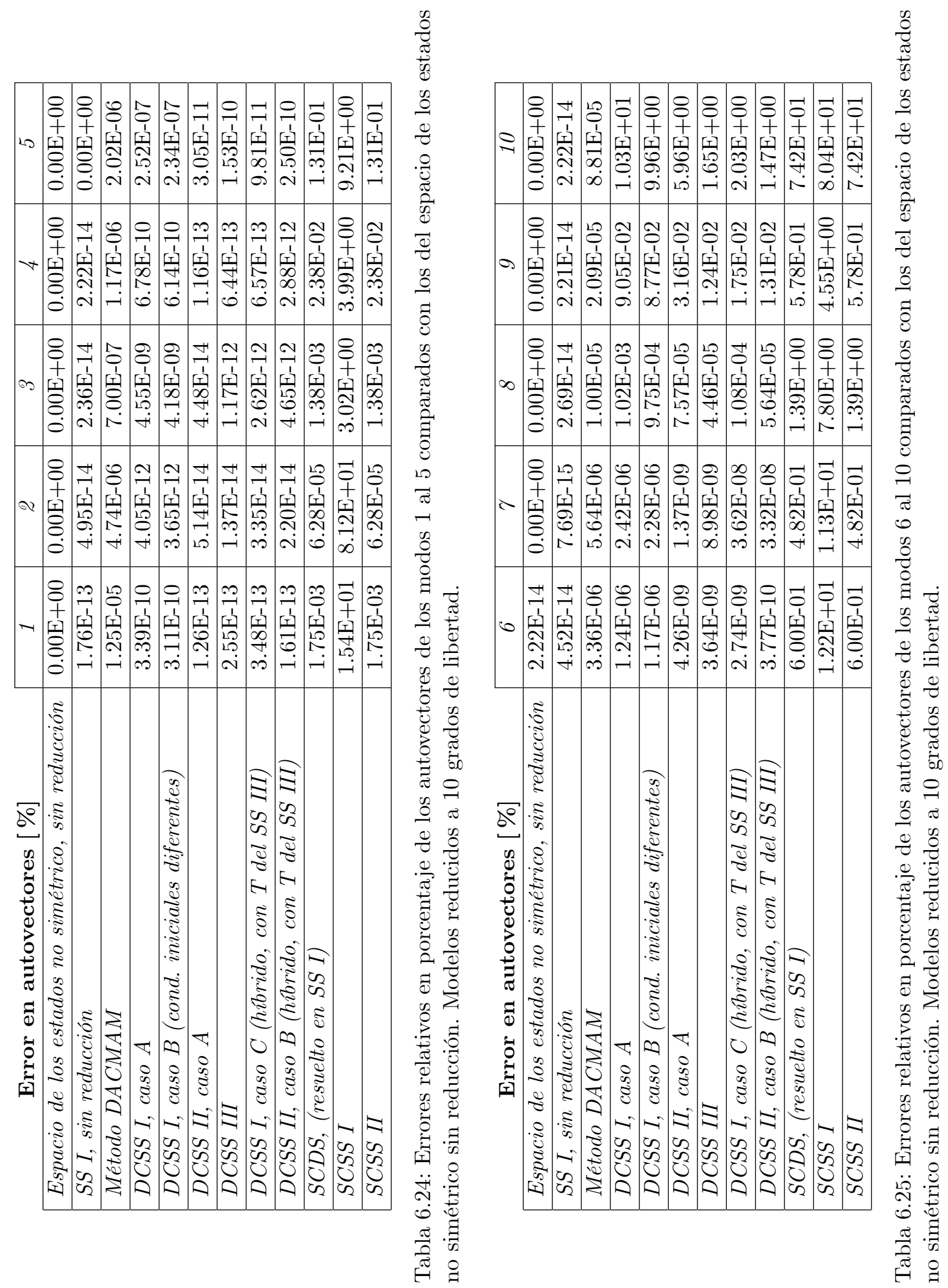
Se puede observar, a partir de los resultados, que a medida que aumenta el número de grados de libertad maestros, son necesarias más iteraciones para cumplir con la tolerancia impuesta para los métodos iterativos. Se muestran las iteraciones correspondientes al equipo más nuevo y potente de los 3 con los que se han realizado las pruebas; las diferencias entre el número de iteraciones entre equipos se atribuye al error de coma flotante de los procesadores, ya que los resultados muestran un número de iteraciones levemente mayor en los equipos más antiguos, pero el resultado numérico final coincide en todos los casos (los códigos y el software empleado para los análisis es el mismo en todos los equipos).

Por contra, en el método $D A C M A M$ con truncatura modal, aunque la precisión es algo peor para los primeros modos, la precisión se mantiene constante independientemente del número de modos que se empleen. Por tanto, para grandes modelos, donde el número de grados de libertad que se retienen habitualmente con los métodos de condensación es sensiblemente mayor a unas pocas unidades, el método muestra su superioridad en la calidad de los resultados.

También se observa que los métodos de condensación estática, aunque proporcionan tiempos de computación menores, producen resultados con errores mucho mayores, por lo que son útiles únicamente como primera aproximación o para problemas sometidos a cargas casi estáticas.

De los métodos de condensación dinámica considerados, no todos muestran los mismos resultados, siendo los correspondientes al espacio de los estados III o los métodos híbridos con la matriz de transformación de coordenadas del espacio III, los que proporcionan menores errores.

La calidad de los resultados y el tiempo de computación también dependen de la tolerancia impuesta para la condición de convergencia. La empleada para esta tesis atiende sólo a la diferencia relativa de los autovalores entre iteraciones, pero se pueden establecer condiciones sobre los autovectores, cuya convergencia es más lenta ya que los autovalores convergen a un ritmo cuadrático frente a los autovectores (Montáns y Muñoz [2013]), o condiciones mixtas sobre ambos. Se ha elegido un valor ( tolerancia $=10^{-5}$ ) que proporciona resultados de buena calidad sin penalizar en exceso el tiempo de computación. Este valor permite que el error producido por el mejor de los métodos de condensación y el método DACMAM sean comparables cuando el número de grados de libertad maestros que se seleccionan son 5 o 10 del total de los 41 grados de libertad del modelo. Si la tolerancia fuese mayor, los métodos iterativos proporcionarían re- 
sultados peores en un tiempo menor. Por contra, si la tolerancia fuese menor, proporcionarían mejores resultados pero con tiempos de proceso mayores. 


\subsection{Parametrizaciones del problema.}

Para mostrar la potencia y bondades del método se realizarán varias parametrizaciones sobre la estructura mencionada.

En las parametrizaciones consideradas se buscará minimizar la respuesta de la estructura, ya sea en desplazamientos, velocidades o aceleraciones, ante la carga del terremoto descrita anteriormente o estudiar su valor máximo. En dicha búsqueda del óptimo, el nodo seleccionado para supervisar la respuesta es la planta 40 del edificio.

Otro motivo para realizar este procedimiento en la búsqueda del óptimo es que la dimensión del problema permite de un modo muy sencillo validar los resultados con un método de integración numérico. Se integran las ecuaciones de equilibrio mediante un algoritmo implícito Newmark-beta con la regla trapezoidal de integración (dando tiempos de computación por el tamaño del problema un orden menor al correspondiente a la obtención de los resultados modales). Este método es incondicionalmente estable. En el caso de un modelo real, cuyo número de grados de libertad es varios órdenes superior al ejemplo propuesto, emplear el algoritmo Newmark-beta para el problema completo haría muy costosa la resolución del problema en términos de tiempos de computación y de volumen de datos generados, más aún al estar parametrizado. El paso de tiempo seleccionado ha sido el correspondiente a la frecuencia de muestreo de la señal de la carga.

En primer lugar, se obtienen por los métodos de reducción descritos la amplitud de la respuesta permanente en cada punto de la transformada discreta de Fourier de la señal del terremoto. Se introducen las amplitudes de la transformada de Fourier para cada frecuencia en un rango que va desde $0,01 \mathrm{~Hz}$ hasta $25 \mathrm{~Hz}$ con un paso en frecuencia de $0,012 \mathrm{~Hz}$.

Adicionalmente, emplear la transformada discreta de Fourier como entrada para el estudio de la respuesta en frecuencia de la estructura implica tener en cuenta ciertos aspectos. Si la densidad de puntos de dicha transformada discreta no es suficiente para captar los picos de la respuesta máxima en frecuencia, añadir puntos no es trivial. La solución habitual es aumentar el número de puntos de la señal temporal añadiendo ceros al final como paso previo a la evaluación de la transformada, pero esto implica una densidad uniforme de puntos, con muchos puntos en zonas con poca información relevante.

Los datos del terremoto disponibles tienen una frecuencia de muestreo de $50 \mathrm{~Hz}$, lo que hace 
que por el teorema de Nyquist no tengan sentido físico las respuestas con frecuencias superiores a $25 \mathrm{~Hz}$. Las amplitudes de las frecuencias altas son en general muy pequeñas y en muchas ocasiones se emplea una frecuencia de corte a partir de la cual se desprecia la excitación.

En el caso de realizar parametrizaciones, si la sensibilidad de la respuesta del sistema al dato parametrizado es pequeña, se debe tener una precaución adicional al introducir una frecuencia de corte, puesto que la pequeña contribución de las frecuencias altas puede influir determinantemente en la identificación del parámetro óptimo. En cualquier caso, esta situación no debe ser habitual, ya que el parámetro seleccionado para modificar la respuesta dinámica no sería adecuado.

Se han empleado tres modos de combinar los datos que se muestran a continuación:

- Aunque el método DACMAM y los métodos de condensación permiten obtener la respuesta transitoria por superposición modal usando como datos de partida las amplitudes y las fases de la transformada discreta de Fourier, se propone un método alternativo para encontrar los parámetros óptimos pero que no permite conocer la respuesta real (los resultados obtenidos deben ser considerados desde el punto de vista cualitativo pero no cuantitativo). Este método, que sólo emplea la información de las amplitudes, proporciona rapidez en la parametrización y sencillez en la implementación, y emplea una herramienta propia de análisis espectral para la combinación de las respuestas permanentes obtenidas por los métodos de reducción comentados sin necesidad de evaluar la solución en el tiempo o realizar una transformada inversa de Fourier. En análisis espectrales se emplean para sumar la respuesta de cada modo, mientras que aquí se usan para sumar las respuestas en frecuencias a la transformada discreta de Fourier de la carga. Se insiste en que el resultado no tiene valor desde el punto de vista cuantitativo, pero permite la búsqueda del óptimo. Se han contemplado 4 combinaciones comunes de entre la gran variedad disponible: el valor máximo en frecuencias (MAX, no es propiamente una combinación pero se incluye aquí a modo de comparación), la raíz de la suma de cuadrados (SRSS), la suma absoluta (ASM) y la raíz de la suma correlada (TPM) (Montáns y Muñoz [2013]):

$$
M A X: A_{c o m b}=\operatorname{máx}\left(A_{i}\right)
$$




$$
\begin{gathered}
S R S S: A_{\text {comb }}=\sqrt{\sum_{i=1}^{M} A_{i}^{2}} \\
A S M: A_{\text {comb }}=\sum_{i=1}^{M} A_{i} \\
T P M: A_{\text {comb }}=\sqrt{\sum_{i=1}^{M} A_{i}^{2}+\sum_{i=1}^{M} \sum_{j=1}^{M} A_{i} A_{j}}
\end{gathered}
$$

Se muestran en la tabla (6.26) los resultados que ofrecen estos métodos comparados con el valor máximo de la respuesta temporal integrando las ecuaciones con un método implícito, Newmark-beta. Se ha comprobado que los resultados cuyo valor se aproxima más a los resultados del algoritmo Newmark-beta se obtienen con el método SRSS, aunque lo que interesa son los resultados que cualitativamente son mejores para ser usados como herra-

\begin{tabular}{|c|c|c|c|c|c|}
\hline Método combinación análisis espectral & $M A X$ & $A S M$ & $S R S S$ & $T P M$ & Newmark-beta \\
\hline Desplazamiento en planta $05[\mathrm{~m}]$ & 0.0379 & 0.2698 & 0.0475 & 0.2698 & 0.0858 \\
\hline Desplazamiento en planta $40[\mathrm{~m}]$ & 0.2081 & 0.9321 & 0.2487 & 0.9321 & 0.4062 \\
\hline Aceleración en planta $05\left[\mathrm{~m} / \mathrm{s}^{2}\right]$ & 0.0579 & 15.6819 & 0.5189 & 15.6819 & 4.3123 \\
\hline Aceleración en planta $40\left[\mathrm{~m} / \mathrm{s}^{2}\right]$ & 0.2232 & 15.5627 & 0.5887 & 15.5627 & 3.4989 \\
\hline
\end{tabular}
mienta para la búsqueda de los parámetros óptimos, y se ve en los siguientes apartados que depende de la parametrización elegida.

Tabla 6.26: Valores máximos de desplazamientos y aceleraciones obtenidos por métodos de combinación de análisis espectral.

También se ha comprobado que el método sugerido, aunque aproximado, es un método consistente, i.e. independiente del número de puntos que tenga la transformada de Fourier en el rango de frecuencia considerado.

- En segundo lugar se ha utilizado la transformada inversa de Fourier, en su implementación 
de MATLAB: transformada inversa rápida de Fourier (iFFT). Este método es mucho más adecuado que el anterior al proporcionar valores que aproximan los reales aunque se obvia la parte transitoria de la respuesta, pero requiere tiempos de computación, que aunque razonables (los algoritmos que emplea son muy eficientes), son mayores.

Se ha implementado de modo que permita obtener la inversa de datos en un rango de frecuencias truncado, procedimiento habitual en el cálculo estructural. Para ello se debe tener especial cuidado, añadiendo ceros en las frecuencias truncadas cercanas al cero y escalando adecuadamente la señal en función de la relación del número de términos que se tienen en cuenta.

También hay que señalar que es necesario adaptar los dos sumatorios de la Ec. (2.55), uno en senos y otro en cosenos, para realizar la transformada inversa y obtener la respuesta temporal. Un método es convertir el sumatorio de senos en otro sumatorio de cosenos equivalente sumando un desfase de $\frac{\pi}{2}$ al ángulo.

Se muestran a modo de ejemplo en una misma figura, para las plantas 5 y 40 del edificio, los resultados en desplazamientos y en aceleraciones que ofrece la transformada inversa con el modelo completo, con el método DACMAM (estas dos primeras son coincidentes como se ha comentado en la descripción del método), con una condensación dinámica de 10 nodos retenidos (la que mejor resultados ofrece: DCSS II, caso B (híbrido, con T del SS III)), e integrando las ecuaciones en el tiempo con el algoritmo Newmark-beta (figuras 6-1, 6-2, 6-3, 6-4). Se ha empleado en estos ejemplos una frecuencia de corte de $14 H z$, un $\Delta f_{\text {análisis }}=0,006 \mathrm{~Hz} \quad \mathrm{y}$ un amortiguamiento del TMD $\quad c_{T M D}=5095 \mathrm{Ns} / \mathrm{m}$.

Este método presenta errores debido al hecho de que la transformada es discreta. También introduce error no poder aplicar la transformada inversa al término transitorio por no ser posible despejar el término del tiempo en el sumatorio, Ec. (2.56). En la presente tesis no se ha profundizado en los diversos métodos para la mejora de los resultados que proporciona la transformada inversa de Fourier, pero es importante señalar que la señal se puede tratar para reducir los errores mencionados, y se muestra un ejemplo de la mejora de los resultados en la figura (6-5), para la cual se han añadido ceros al final de la señal del terremoto, pero que implica un aumento en el tiempo de ejecución.

- Por último, también se ha implementado la combinación de resultados mediante la eva- 


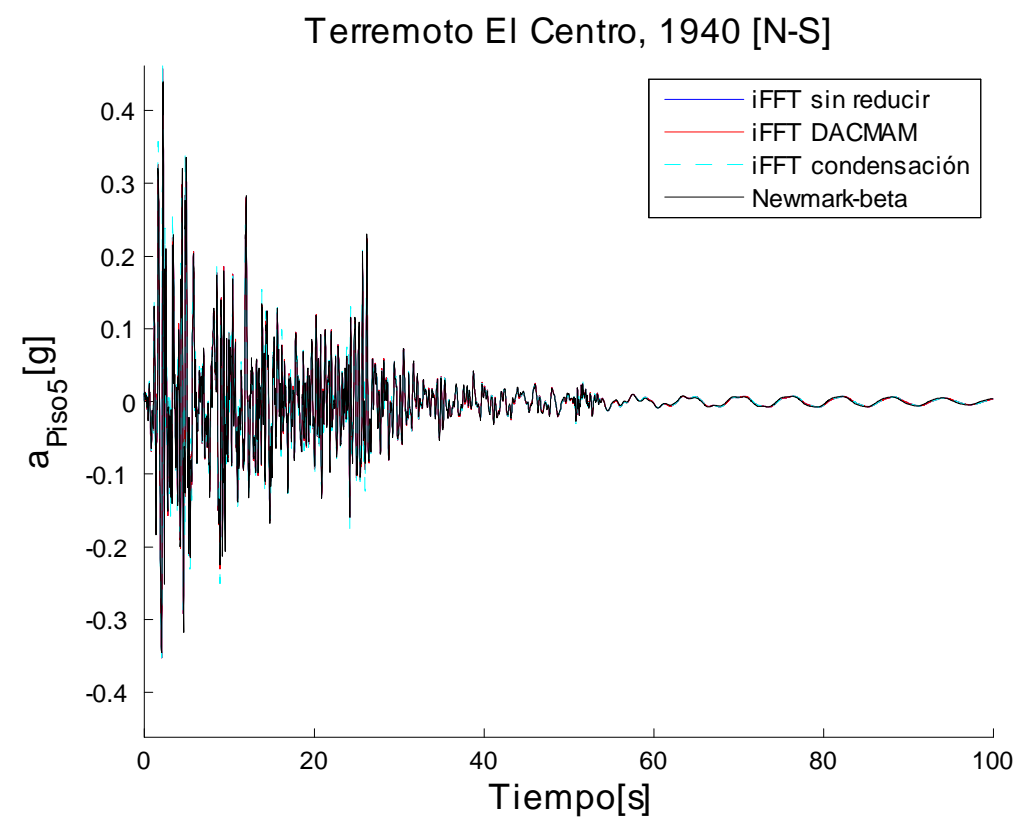

Figura 6-1: Respuesta en aceleración de la planta 5 del edificio a la carga del terremoto. Comparativa de resolución por diversos métodos.

luación temporal de los sumandos de los términos en frecuencia. Este método, aunque más exacto que el anterior al permitir evaluar la componente transitoria, implica tiempos de proceso mucho mayores incluso para un problema reducido como el que se emplea en la tesis. Sólo se muestran resultados de este método a modo de comparación en este apartado, en las figuras (6-6, 6-7, 6-8 y 6-9), donde se puede ver la contribución diferenciada de las parte transitoria y permanente de la solución (ecuaciones 2.55 y 2.56).

Se observa en los ejemplos mostrados que los datos de las combinaciones por métodos espectrales ofrecen los resultados de peor calidad, la evaluación temporal del sumatorio de términos en frecuencia ofrece resultados excelentes, y la transformada inversa de Fourier da lugar a resultados también excelentes siempre y cuando el término transitorio despreciado no sea relevante. Que el término transitorio sea relevante o no depende de varios factores: la respuesta que estemos considerando (desplazamientos, velocidades o aceleraciones), el piso en el que se mida la respuesta, y el amortiguamiento presente en la estructura (como es lógico, a menor amortiguamiento mayor importancia de los términos transitorios). En el caso de la respuesta en 


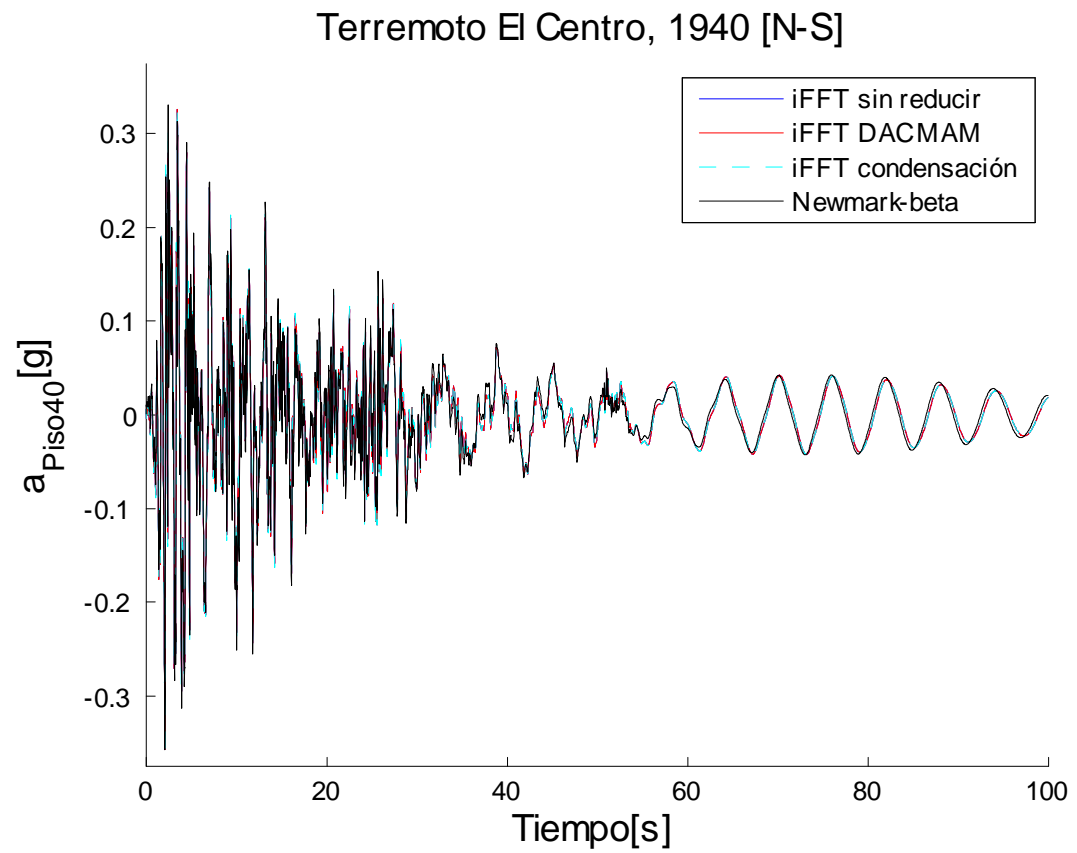

Figura 6-2: Respuesta en aceleración de la planta 40 del edificio a la carga del terremoto. Comparativa de resolución por diversos métodos.

desplazamientos, los resultados se pueden considerar coincidentes a excepción del error que se produce en la evolución temporal debido a la truncatura de los términos en frecuencia con un máximo de $14 \mathrm{~Hz}$ en lugar de los $25 \mathrm{~Hz}$ disponibles en la transformada discreta de Fourier de la carga y debido a la densidad de puntos de la transformada. En el caso de las aceleraciones, se comprueba por las figuras (6-2 y 6-9) que el error que se aprecia entre la solución a partir de la transformada inversa de Fourier y la solución obtenida por integración numérica se debe principalmente a los términos transitorios de la respuesta. De todos modos, a la vista del pequeño error en los resultados, la respuesta para la carga del terremoto se puede considerar básicamente como respuesta permanente y los términos transitorios se pueden obviar, por lo que los resultados proporcionados por la transformada inversa de Fourier serán óptimos en el presente caso.

Cabe destacar que no se ha aplicado una corrección de base cero a los desplazamientos para compensar el hecho de que habitualmente la integral de las aceleraciones de un terremoto no suele ser nula. El registro temporal de la carga del terremoto que se emplea comienza con un 
Terremoto El Centro, 1940 [N-S]

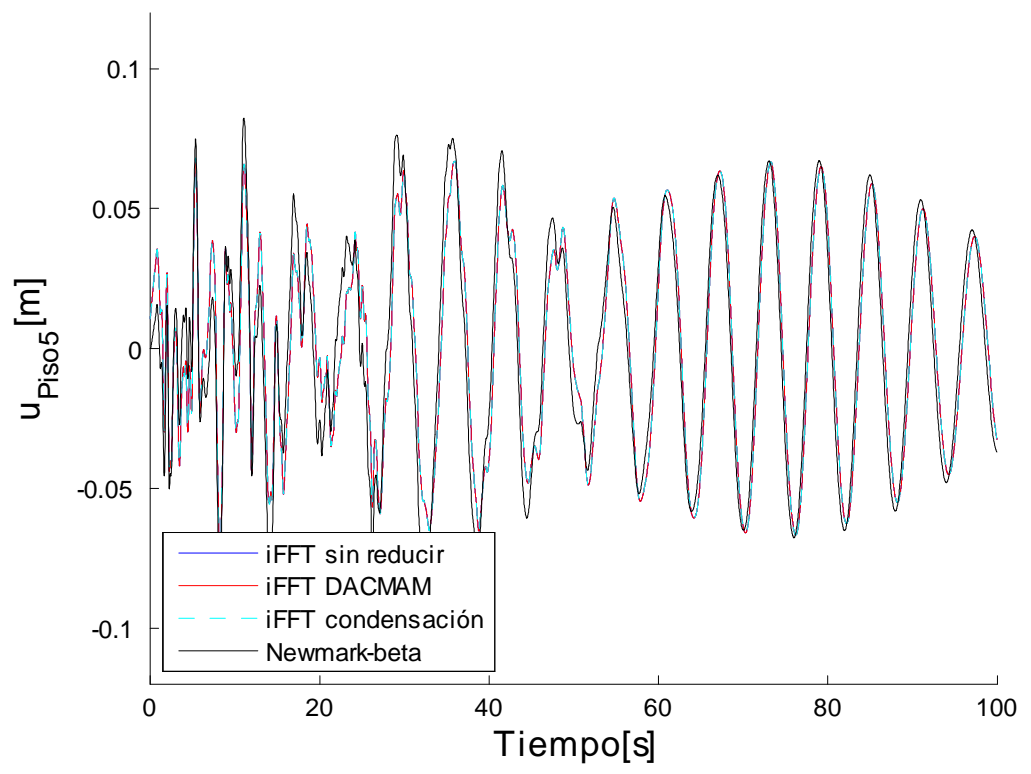

Figura 6-3: Respuesta en desplazamientos de la planta 5 del edificio a la carga del terremoto. Comparativa de resolución por diversos métodos.

valor de $a_{g}=-1,41 \cdot 10^{-2} \mathrm{~m} / \mathrm{s}^{2}$. 
Terremoto El Centro, 1940 [N-S]

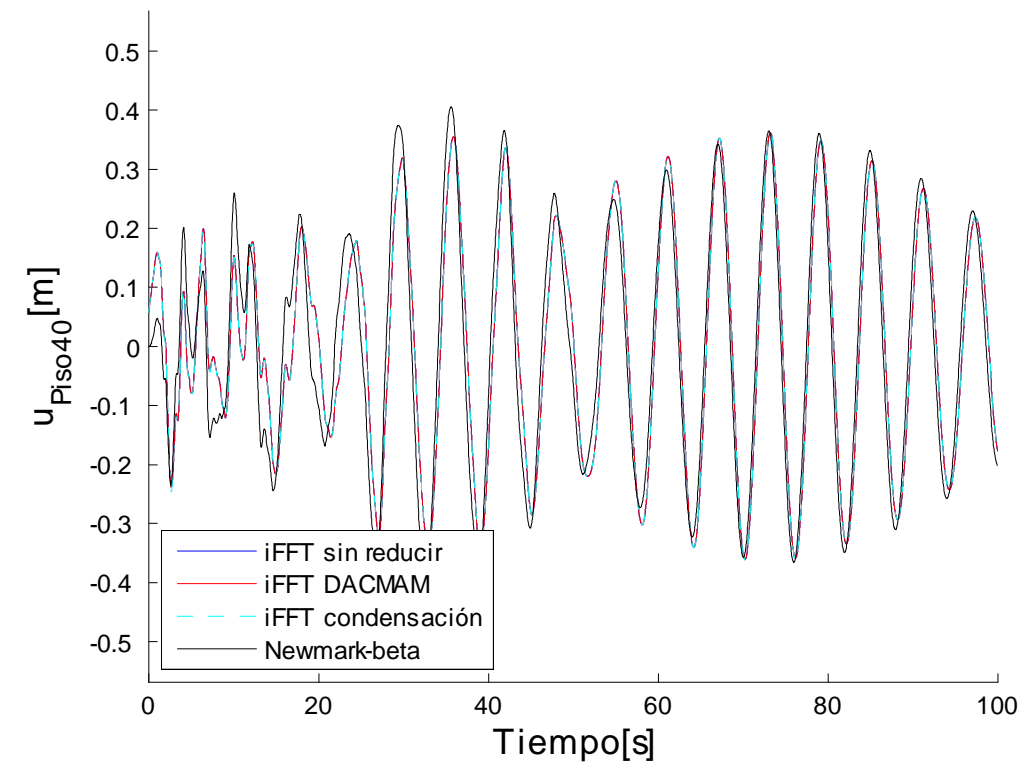

Figura 6-4: Respuesta en desplazamientos de la planta 40 del edificio a la carga del terremoto. Comparativa de resolución por diversos métodos. 


\section{Terremoto El Centro, 1940 [N-S]}

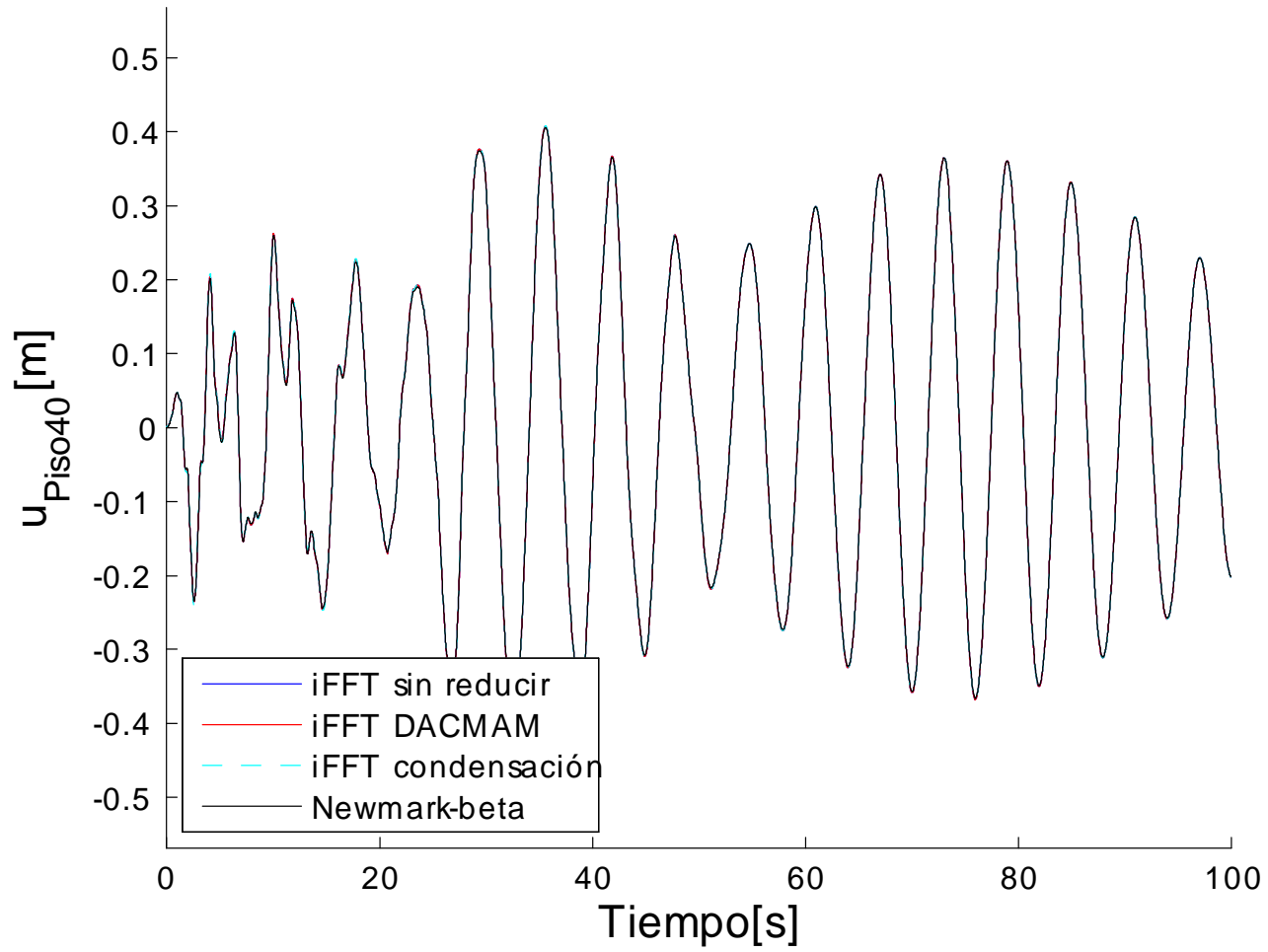

Figura 6-5: Respuesta en desplazamientos de la planta 40 del edificio a la carga del terremoto (prolongada en el tiempo con valor nulo para mejorar resultados de la transformada de Fourier). Comparativa de resolución por diversos métodos. 


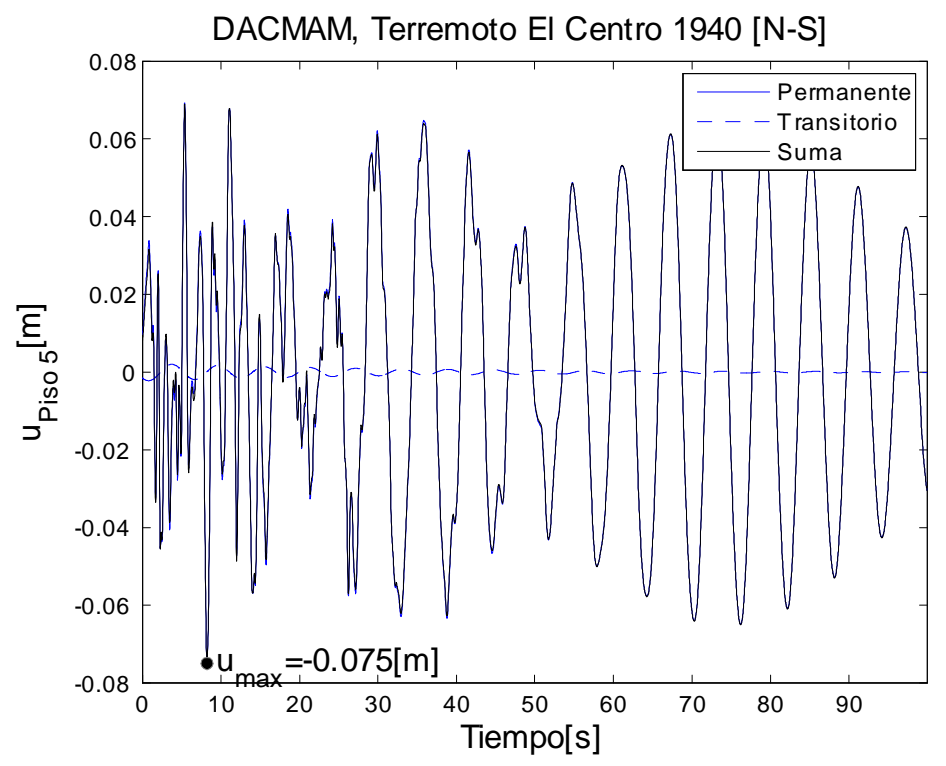

Figura 6-6: Respuesta en desplazamientos transitoria y permanente a la carga del terremoto para la planta 5 del edificio, evaluando la solución en sumatorio de senos con el método DACMAM.

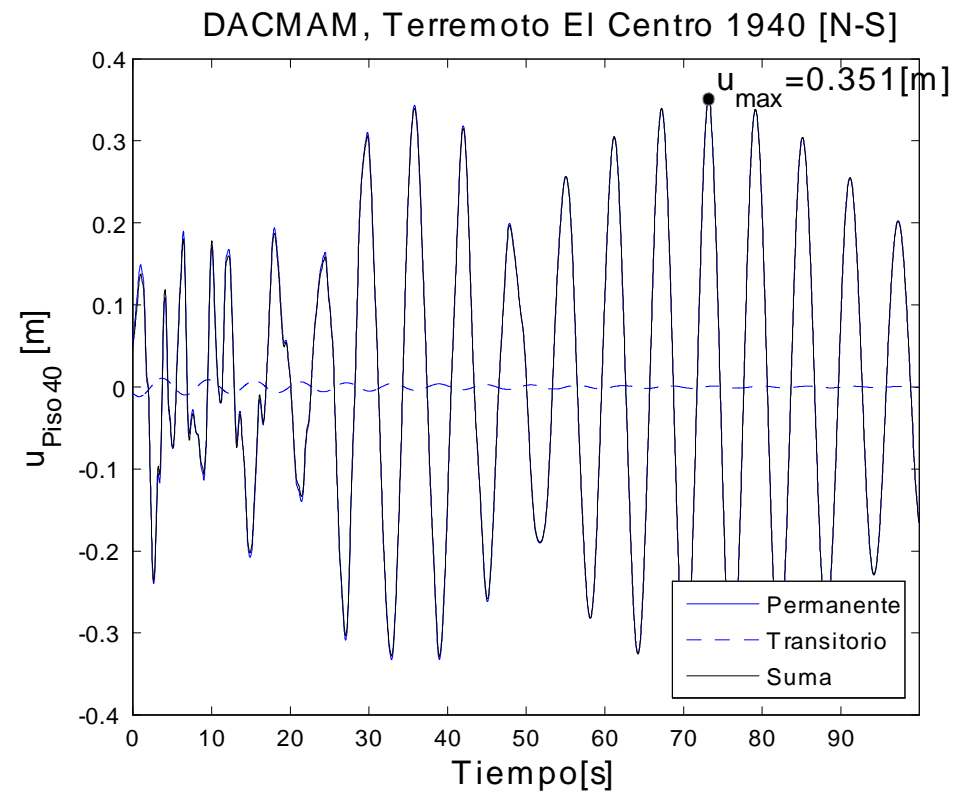

Figura 6-7: Respuesta en desplazamientos transitoria y permanente a la carga del terremoto para la planta 40 del edificio, evaluando la solución en sumatorio de senos con el método DACMAM. 


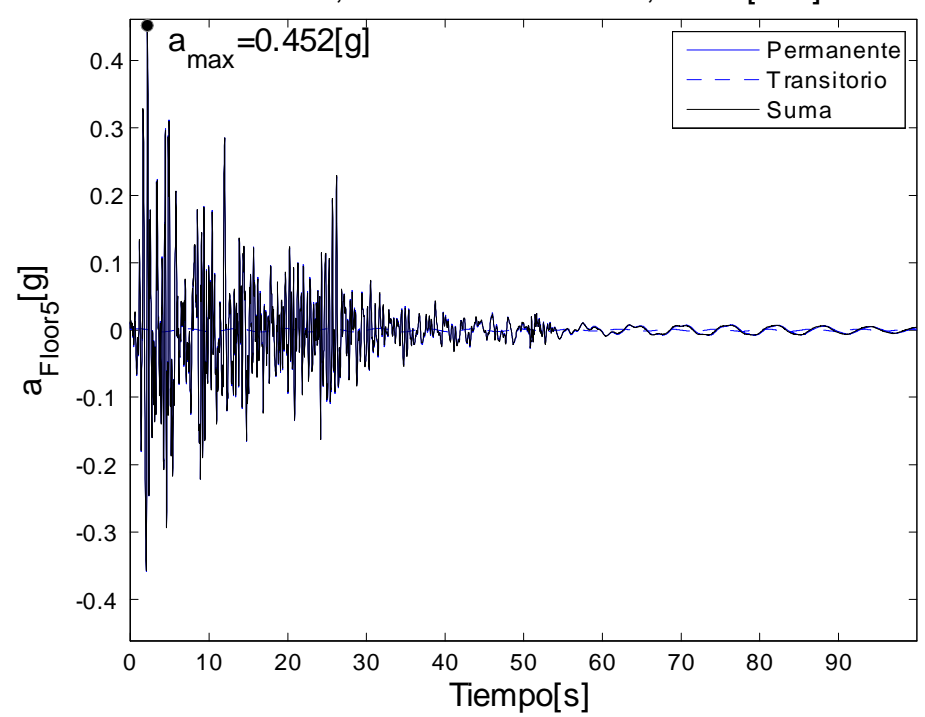

Figura 6-8: Respuesta en aceleraciones transitoria y permanente a la carga del terremoto para la planta 5 del edificio, evaluando la solución en sumatorio de senos con el método DACMAM.

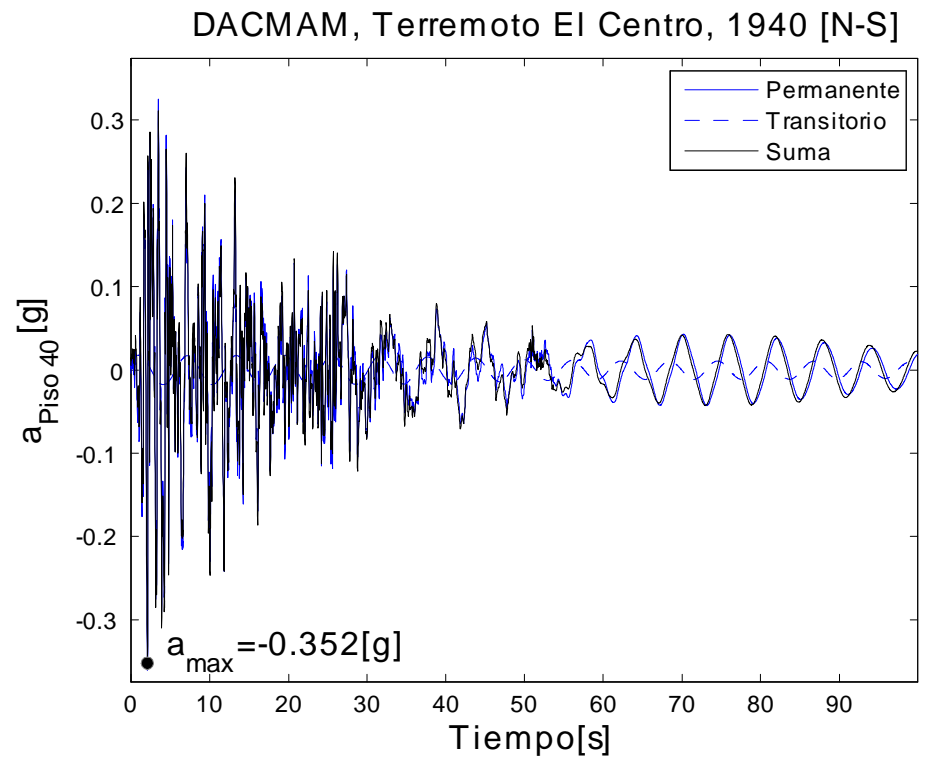

Figura 6-9: Respuesta en aceleraciones transitoria y permanente a la carga del terremoto para la planta 40 del edificio, evaluando la solución en sumatorio de senos con el método DACMAM. 


\subsubsection{Parametrización de rigidez del TMD para minimizar desplazamientos}

La primera parametrización considerada es la búsqueda de la rigidez que hace mínima la respuesta en desplazamientos en la última planta del edificio ante la carga del terremoto. Se trata de validar que el TMD sintonizado a la primera frecuencia del edificio es el valor óptimo para el terremoto. Como se ha comentado, se parte de la respuesta permanente en frecuencias tomando como excitación las amplitudes dadas por la transformada discreta de Fourier del terremoto, que se ha refinado añadiendo ceros al final de la señal original para obtener un $\Delta f_{\text {análisis }}=0,006 \mathrm{~Hz} \quad$ (se puede ver en la tabla (6.30) cómo se incrementan los tiempos de computación por añadir puntos al análisis modal). Para esta parametrización, el método de mezcla de respuestas en frecuencias que proporciona el mejor resultado es el SRSS, aunque el método TPM da resultados similares (se insiste en que sólo se emplea esta mezcla de modo cualitativo para determinar el parámetro óptimo).

Se muestran superpuestas en la figura (6-10) las respuestas de desplazamientos en frecuencia a la carga del terremoto con el problema completo en el espacio no simétrico y con el método $D A C M A M$ para la planta $40 \mathrm{y}$ el valor óptimo de rigidez, $k_{T M D}=260570 \mathrm{~N} / \mathrm{m}$.

Se muestra asimismo la respuesta en desplazamientos (figura 6-11), en el mismo punto, calculado con el algoritmo implícito mencionado, para el parámetro de amortiguamiento óptimo y calculado empleando la transformada inversa de Fourier con el modelo completo, con el método $D A C M A M$ y con una condensación a $5 \mathrm{gdl}$ (en todos los casos se ha empleado, a la vista de las pruebas de respuesta en frecuencia, una frecuencia de corte de $f_{\text {máx }}=8 \mathrm{~Hz}$ ).

Se ha variado la rigidez del TMD entre $k_{T M D}=1 \cdot 10^{3} \mathrm{~N} / \mathrm{m} \quad$ y $\quad k_{T M D}=6 \cdot 10^{5} \mathrm{~N} / \mathrm{m}$. Se han tomado en dicho rango 31 valores equiespaciados. En la figura (6-12) se muestra cómo en el rango seleccionado se encuentra un mínimo de la respuesta buscada y dicho mínimo.se capta de manera adecuada con la inversa de Fourier de los métodos modales.

El valor de rigidez que para la carga del terremoto proporciona un menor valor en desplazamientos en la planta 40 del edificio, $k_{T M D}^{\text {óptimo }}=260570 \mathrm{~N} / \mathrm{m}$ no se corresponde con el valor del ejemplo inicial, $\quad k_{T M D}=300900 N / m$, que corresponde al valor óptimo para otro tipo de carga (por ejemplo una carga de viento con contenido en frecuencia a $f=0,172 \mathrm{~Hz}$ ).

En la tabla (6.27) se muestran los valores óptimos del parámetro $k_{T M D}$ en función del método empleado con las herramientas mencionadas. Los métodos de reducción retienen sólo 5 


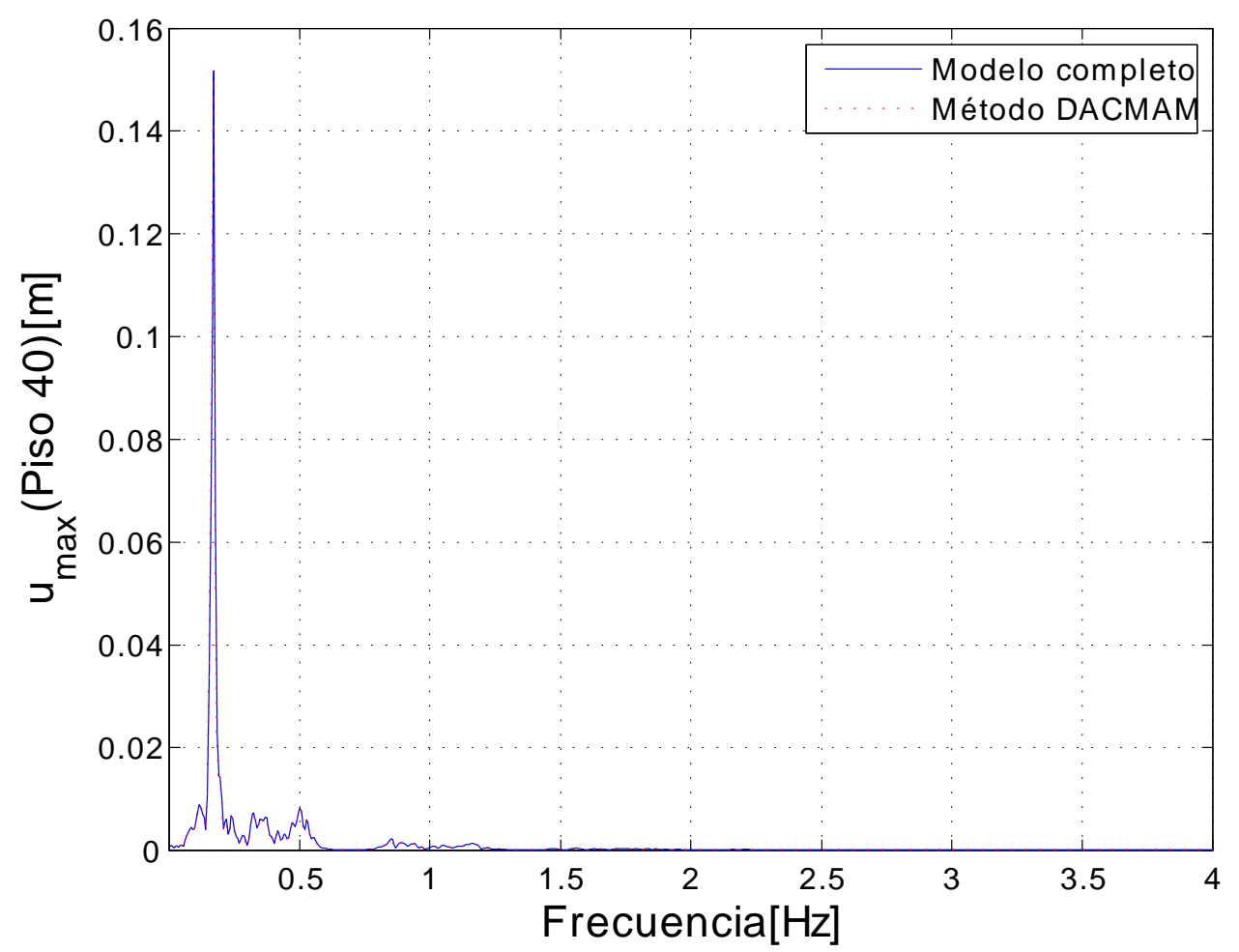

Figura 6-10: Resultados de desplazamiento de la planta 40, respuestas en frecuencia. Comparación de métodos: modelo completo en el espacio de lo estados no simétrico y método DACMAM sin truncatura modal.

grados de libertad, correspondientes a los grados de libertad de las plantas 10, 20, 30, 40 y al TMD.

Se observa en la tabla que los tres métodos de combinación espectral, aunque no proporcionan el valor de amortiguamiento óptimo de los 31 valores considerados, sí proporcionan un valor muy próximo (un valor de rigidez adyacente al óptimo de los 31 considerados), por lo que se puede considerar que los resultados son aceptables.

Se muestra por último en la tabla (6.28) los tiempos requeridos en función del método.

Se observa que el método DACMAM proporciona tiempos de computación del mismo orden que las condensaciones, pero proporcionando resultados con la información del modelo completo. La diferencia con los métodos de condensación es levemente perceptible en este ejemplo debido al reducido contenido en frecuencia de la respuesta (figura 6-10), por lo que los métodos de 


\section{Terremoto ElCentro 1940[N-S]}

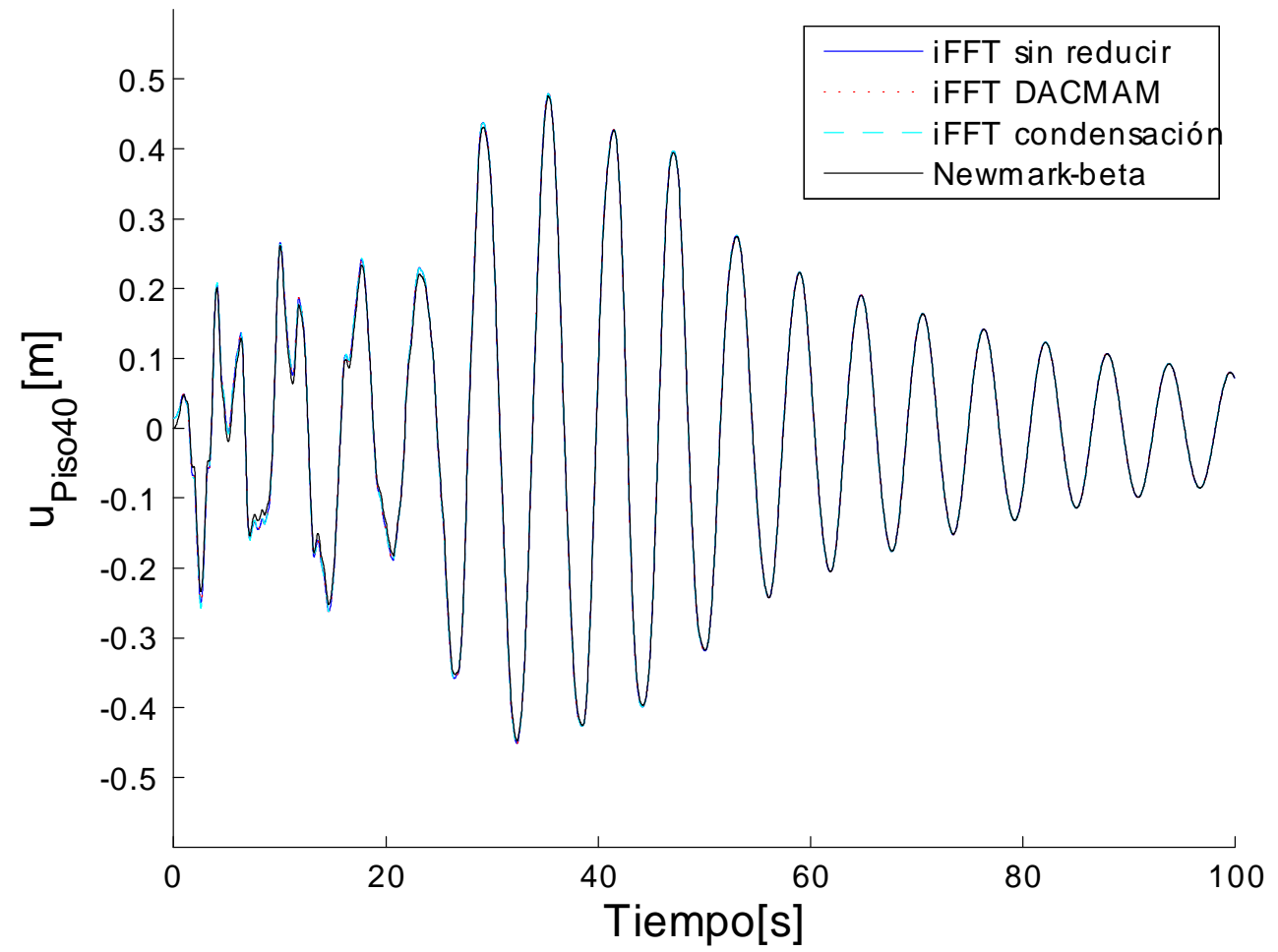

Figura 6-11: Respuesta temporal en desplazamientos de la planta 40 a la carga del terremoto. Caso óptimo de rigidez del TMD para minimizar desplazamientos. Comparación de diversos métodos.

condensación dan muy buenos resultados. Cabe destacar que para grandes estructuras, donde el contenido de la respuesta en frecuencias para la variable supervisada puede ser mucho más disperso, emplear todos los modos proporcionará resultados considerablemente de mayor calidad para los mismos tiempos de proceso. 


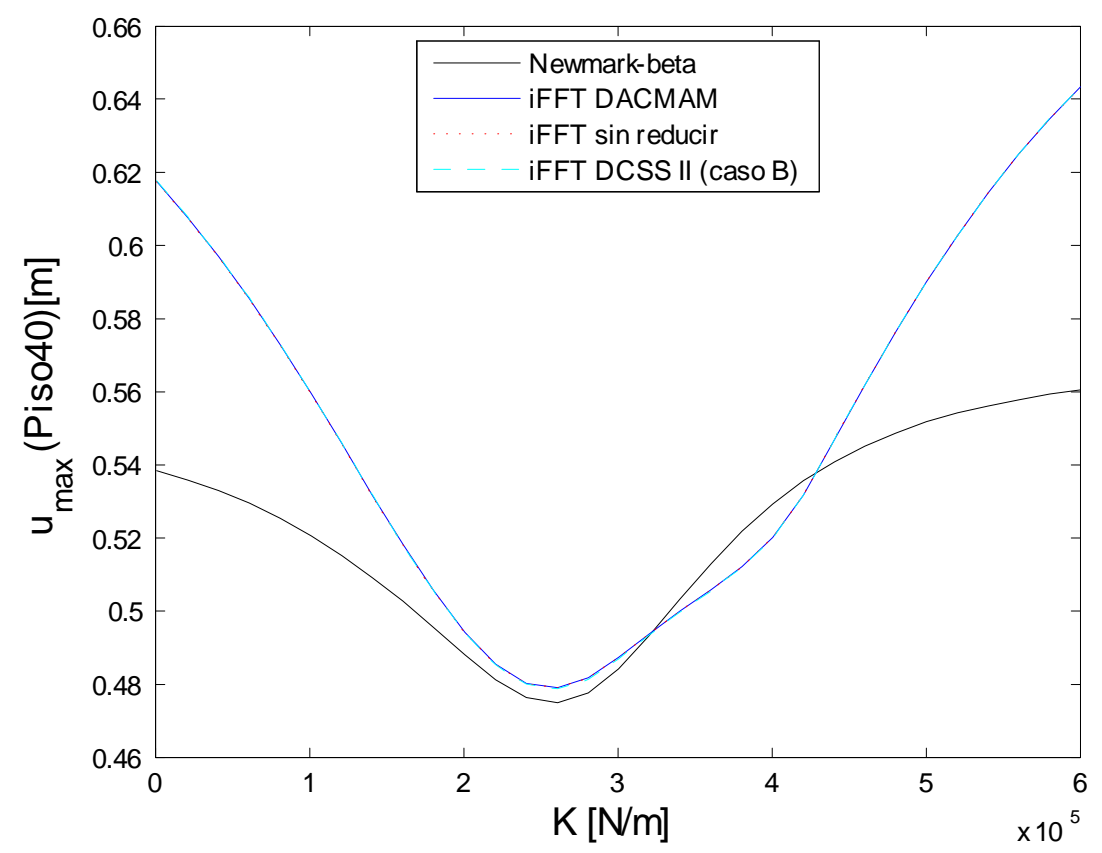

Figura 6-12: Respuesta máxima en desplazamientos de la planta 40 en función del parámetro de rigidez del TMD. Comparación de métodos: algoritmo de integración Newmark-beta, inversa de Fourier con el método DACMAM, inversa de Fourier del modelo completo, y con la condensación DCSS II, caso B (híbrido, con T del SS III).

\begin{tabular}{|c|c|c|c|c|c|}
\hline$k_{T M D}^{\text {óptimo }}[N / m]$ & $i F F T$ & $M A X$ & $A S M$ & $S R S S$ & $T P M$ \\
\hline Espacio de los estados no simétrico, sin reducc. & 260570 & 300500 & 280530 & 280530 & 280530 \\
\hline$S S I$, sin reducción & 260570 & 300500 & 280530 & 280530 & 280530 \\
\hline Método DACMAM con truncatura a 5 modos & 260570 & 300500 & 280530 & 280530 & 280530 \\
\hline Método DACMAM sin truncatura & 260570 & 300500 & 280530 & 280530 & 280530 \\
\hline DCSS I, caso $A$ & 260570 & 300500 & 280530 & 280530 & 280530 \\
\hline DCSS I, caso B (cond. iniciales diferentes) & 260570 & 300500 & 280530 & 280530 & 280530 \\
\hline$D C S S I I$, caso $A$ & 260570 & 300500 & 280530 & 280530 & 280530 \\
\hline DCSS III & 260570 & 300500 & 280530 & 280530 & 280530 \\
\hline DCSS I, caso C (híbrido, con T del SS III) & 260570 & 300500 & 280530 & 280530 & 280530 \\
\hline DCSS II, caso B (híbrido, con T del SS III) & 260570 & 300500 & 280530 & 280530 & 280530 \\
\hline SCDS, (resuelto en $S S I)$ & 260570 & 280530 & 260570 & 260570 & 260570 \\
\hline SCSS I & 600000 & 120800 & 600000 & 600000 & 600000 \\
\hline$S C S S I I$ & 260570 & 280530 & 260570 & 260570 & 260570 \\
\hline Algoritmo de integración Newmark-beta & 260570 & $\mathrm{n} / \mathrm{a}$ & $\mathrm{n} / \mathrm{a}$ & $\mathrm{n} / \mathrm{a}$ & $\mathrm{n} / \mathrm{a}$ \\
\hline
\end{tabular}

Tabla 6.27: Valores óptimos de amortiguamiento del TMD para minimizar la respuesta en aceleraciones ante la carga del terremoto. 


\begin{tabular}{|l|c|c|c|}
\cline { 2 - 4 } \multicolumn{1}{c|}{ tiempos [s] } & Portátil & Estación 2013 & Estación 2007 \\
\hline Espacio de los estados no simétrico, sin reducc. & 858 & 502 & 557 \\
\hline SS I, sin reducción & 814 & 495 & 541 \\
\hline Método DACMAM con truncatura a 5 modos & 127 & 64 & 76 \\
\hline Método DACMAM sin truncatura & 182 & 96 & 183 \\
\hline DCSS I, caso A & 110 & 63 & 157 \\
\hline DCSS I, caso B (cond. iniciales diferentes) & 110 & 63 & 89 \\
\hline DCSS II, caso A & 112 & 64 & 90 \\
\hline DCSS III & 113 & 63 & 86 \\
\hline DCSS I, caso C (híbrido, con T del SS III) & 110 & 63 & 85 \\
\hline DCSS II, caso B (híbrido, con T del SS III) & 110 & 64 & 85 \\
\hline SCDS, (resuelto en SS I) & 106 & 62 & 60 \\
\hline SCSS I & 99 & 62 & 60 \\
\hline SCSS II & 104 & 62 & 60 \\
\hline
\end{tabular}

Tabla 6.28: Tiempos de computación de los diferentes métodos para el caso de parametrización del amortiguamiento del TMD en busca de minimizar aceleraciones. 


\subsubsection{Parametrización de amortiguamiento del TMD para minimizar acele- raciones}

Otra parametrización considerada es la búsqueda del amortiguamiento que hace mínima la respuesta en aceleraciones en la última planta del edificio ante la carga del terremoto. Se parte de la respuesta permanente en frecuencias tomando como excitación las amplitudes dadas por la transformada discreta de Fourier del terremoto, que se ha refinado añadiendo ceros al final de la señal original para obtener un $\Delta f_{\text {análisis }}=0,001 \mathrm{~Hz} \quad$ (se pueden ver en la tabla (6.30) los tiempos de computación resultantes).

Se muestran en la misma figura (6-13) las respuestas en frecuencia de aceleraciones para la planta 40 a la carga del terremoto con el problema completo en el espacio de los estados no simétrico y con el método DACMAM, para el valor óptimo de amortiguamiento: $c=20080 N s / m$.

Se muestra asimismo la respuesta temporal en aceleraciones de la misma planta en la figura (6-14). Están sobreimpresas las curvas correspondientes al método implícito de integración Newmark-beta y empleando la transformada inversa de Fourier con el modelo completo, con el método DACMAM y con una condensación de 5 gdl maestros.

Se ha variado el amortiguamiento del TMD entre $c_{T M D}=1 \cdot 10^{2} \mathrm{Ns} / \mathrm{m} \quad$ y $\quad c_{T M D}=1 \cdot 10^{5} \mathrm{Ns} / \mathrm{m}$. Se han tomado en dicho rango 21 valores equiespaciados. En la figura (6-15) se muestra cómo en el rango seleccionado se encuentra un mínimo de la respuesta buscada, aunque la variación de la respuesta máxima es muy reducida. Esta diferencia no alcanza un $0.03 \%$ entre el máximo y mínimo en el rango considerado de acuerdo a los resultados de la integración numérica; en la figura (6-16) se pueden comparar los resultados obtenidos empleando la transformada inversa de Fourier a partir de la respuesta en frecuencias y los correspondientes a la integración numérica. En las figuras (6-17 y 6-18) se muestra también la misma gráfica para el método DACMAM con las combinaciones SRSS y TPM respectivamente, empleando todos los modos de la estructura.

En la tabla (6.29) se muestran los valores óptimos del parámetro $c_{T M D}$ en función del método empleado con las herramientas mencionadas. En los métodos de condensación se han retenido sólo 5 nodos maestros, correspondientes a los grados de libertad de las plantas 10, 20, 30, 40 y al TMD.

Los métodos SRSS y TPM, aunque no proporcionan el valor de amortiguamiento óptimo 


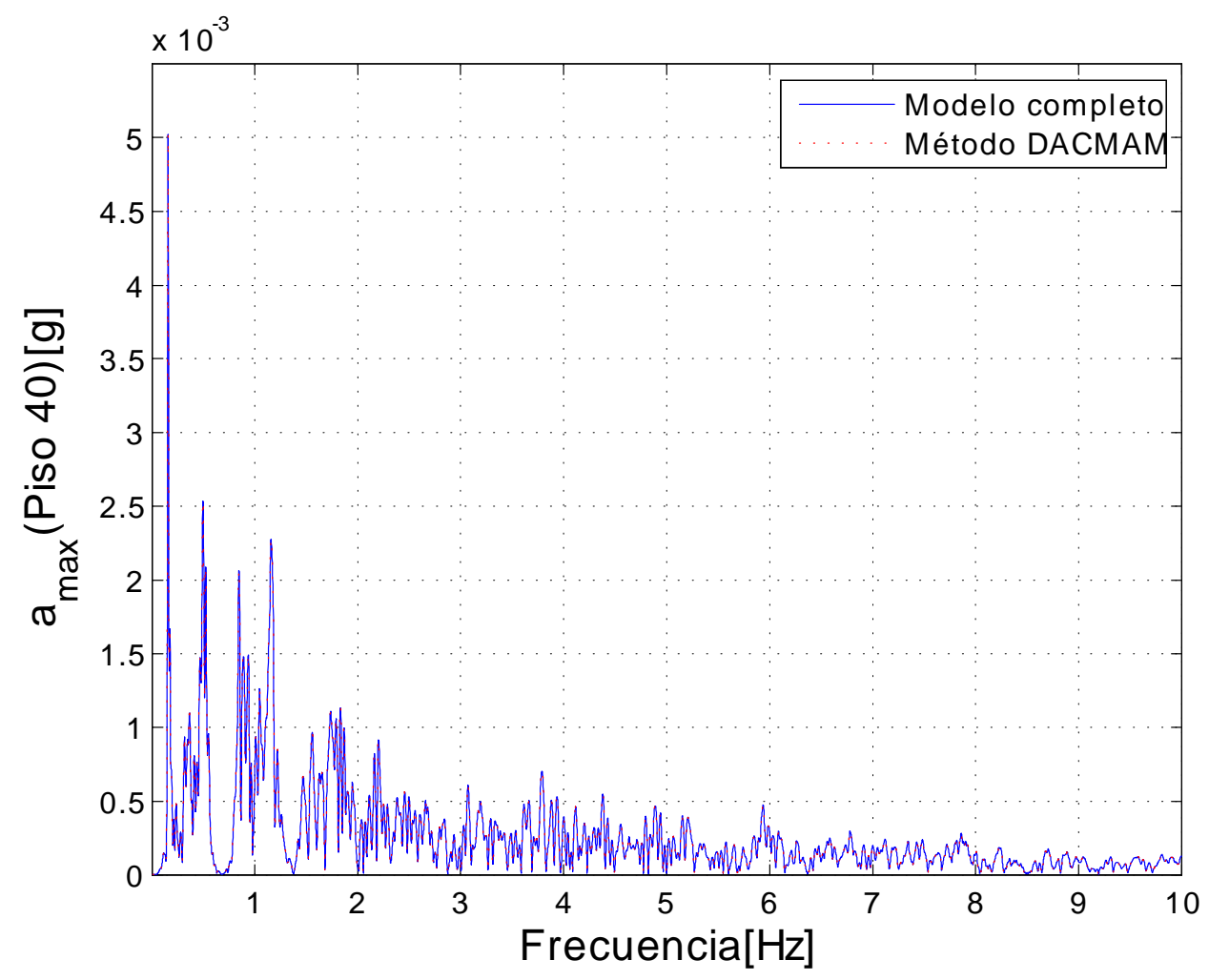

Figura 6-13: Resultados de aceleración de la planta 40, respuestas en frecuencia. Comparación de métodos: modelo completo en el espacio de lo estados no simétrico y método DACMAM sin truncatura modal.

de los 21 valores considerados, sí proporcionan para los métodos de condensación dinámicos y los modelos sin reducción un valor muy próximo, por lo que se puede considerar que los resultados son aceptables. La forma de las figuras (6-17 y 6-18) muestran que también presentan un mínimo en el rango de amortiguamiento considerado, aunque su forma tiene diferencias relevantes respecto a la respuesta máxima real (figura 6-15).

Se muestran por último en la tabla (6.30) los tiempos requeridos en función del método.

Se observa que el método DACMAM proporciona tiempos de computación del mismo orden que las condensaciones, pero proporcionando resultados con la información del modelo completo. En este caso, por la baja sensibilidad de la estructura a cambios de amortiguamiento en el TMD y por los errores debidos a no considerar los términos transitorios de la respuesta (este error es más importante cuanto menor es el amortiguamiento), los resultados no son aceptables para la 


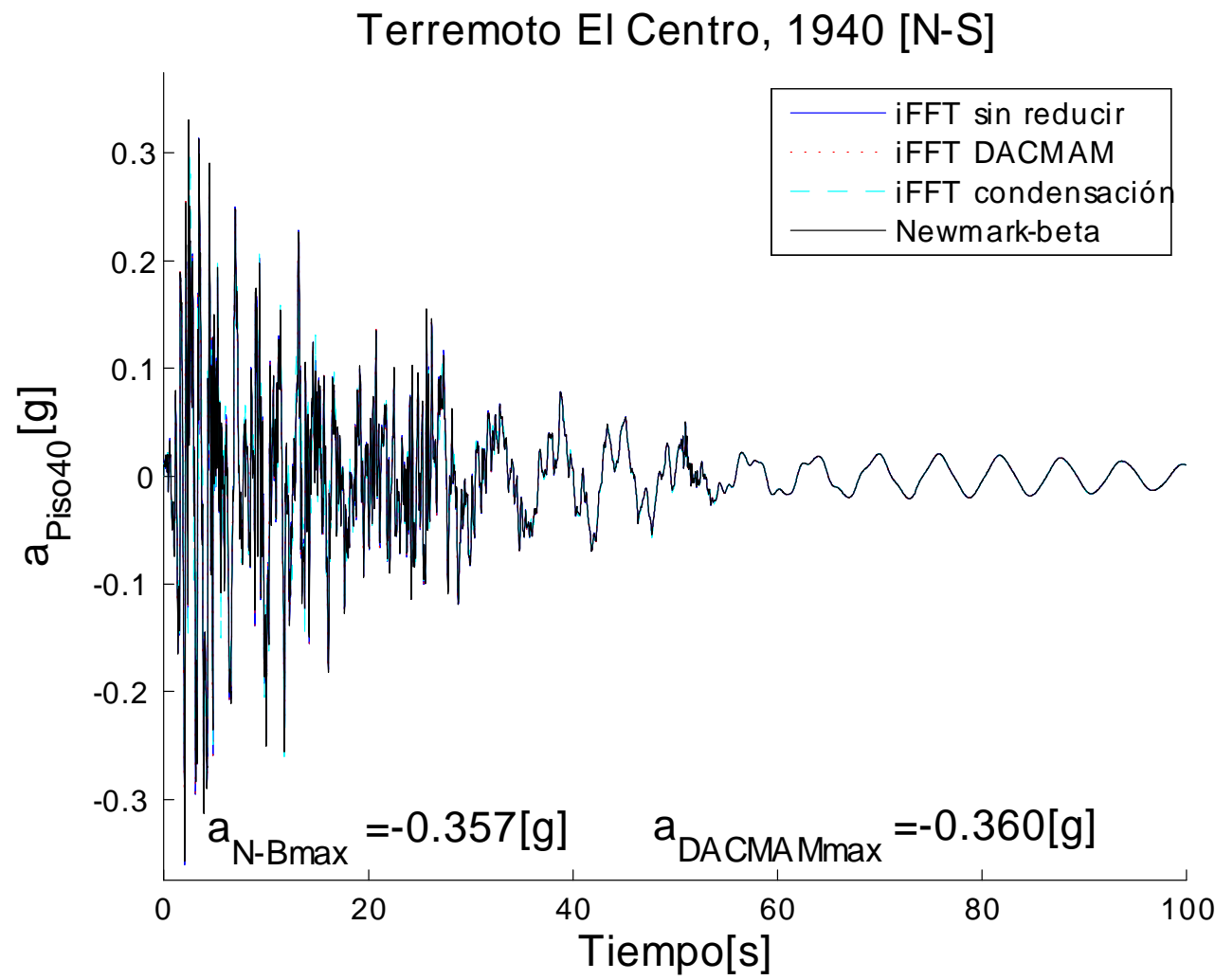

Figura 6-14: Respuesta temporal en aceleraciones de la planta 40 a la carga del terremoto. Caso óptimo de amortiguamiento del TMD para minimizar aceleraciones. Comparación de diversos métodos.

búsqueda del óptimo. Se puede ver en la figura (6-14) que, a pesar de ello, la respuesta temporal es casi idéntica en todos los métodos considerados. 


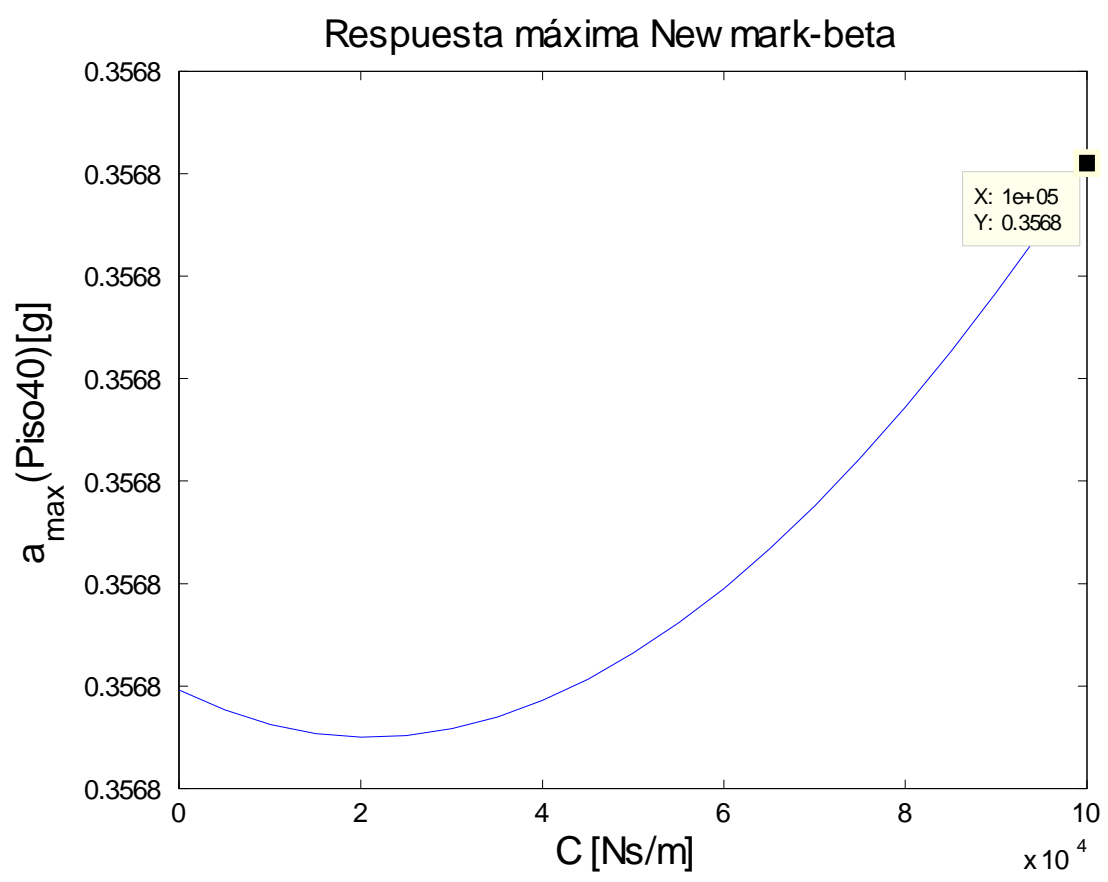

Figura 6-15: Respuesta máxima en aceleraciones de la planta 40 en función del parámetro de amortiguamiento del TMD. Algoritmo de integración Newmark-beta.

\begin{tabular}{|l|c|c|c|c|c|}
\cline { 2 - 6 } \multicolumn{1}{c|}{$c_{T M D}^{\text {óptimo }}[\mathrm{Ns} / \mathrm{m}]$} & iFFT & MAX & ASM & SRSS & TPM \\
\hline Espacio de los estados no simétrico, sin reducc. & 100 & 40060 & 25075 & 30070 & 25075 \\
\hline SS I, sin reducción & 100 & 40060 & 25075 & 30070 & 25075 \\
\hline Método DACMAM con truncatura a 5 modos & 100 & 40060 & 25075 & 30070 & 25075 \\
\hline Método DACMAM sin truncatura & 100 & 40060 & 25075 & 30070 & 25075 \\
\hline DCSS I, caso A & 100 & 40060 & 30070 & 30070 & 30070 \\
\hline DCSS I, caso B (cond. iniciales diferentes) & 100 & 40060 & 25075 & 30070 & 25075 \\
\hline DCSS II, caso A & 100 & 40060 & 25075 & 30070 & 25075 \\
\hline DCSS III & 100000 & 40060 & 25075 & 30070 & 25075 \\
\hline DCSS I, caso C (híbrido, con T del SS III) & 100 & 40060 & 25075 & 30070 & 25075 \\
\hline DCSS II, caso B (híbrido, con T del SS III) & 100 & 40060 & 30070 & 30070 & 30070 \\
\hline SCDS, (resuelto en SS I) & 100 & 40060 & 25075 & 30070 & 25075 \\
\hline SCSS I & 100000 & 100000 & 100000 & 100000 & 100000 \\
\hline SCSS II & 100 & 40060 & 25075 & 30070 & 25075 \\
\hline Algoritmo de integración Newmark-beta & 20080 & $\mathrm{n} / \mathrm{a}$ & $\mathrm{n} / \mathrm{a}$ & $\mathrm{n} / \mathrm{a}$ & $\mathrm{n} / \mathrm{a}$ \\
\hline
\end{tabular}

Tabla 6.29: Valores óptimos de amortiguamiento del TMD para minimizar la respuesta en aceleraciones ante la carga del terremoto. 


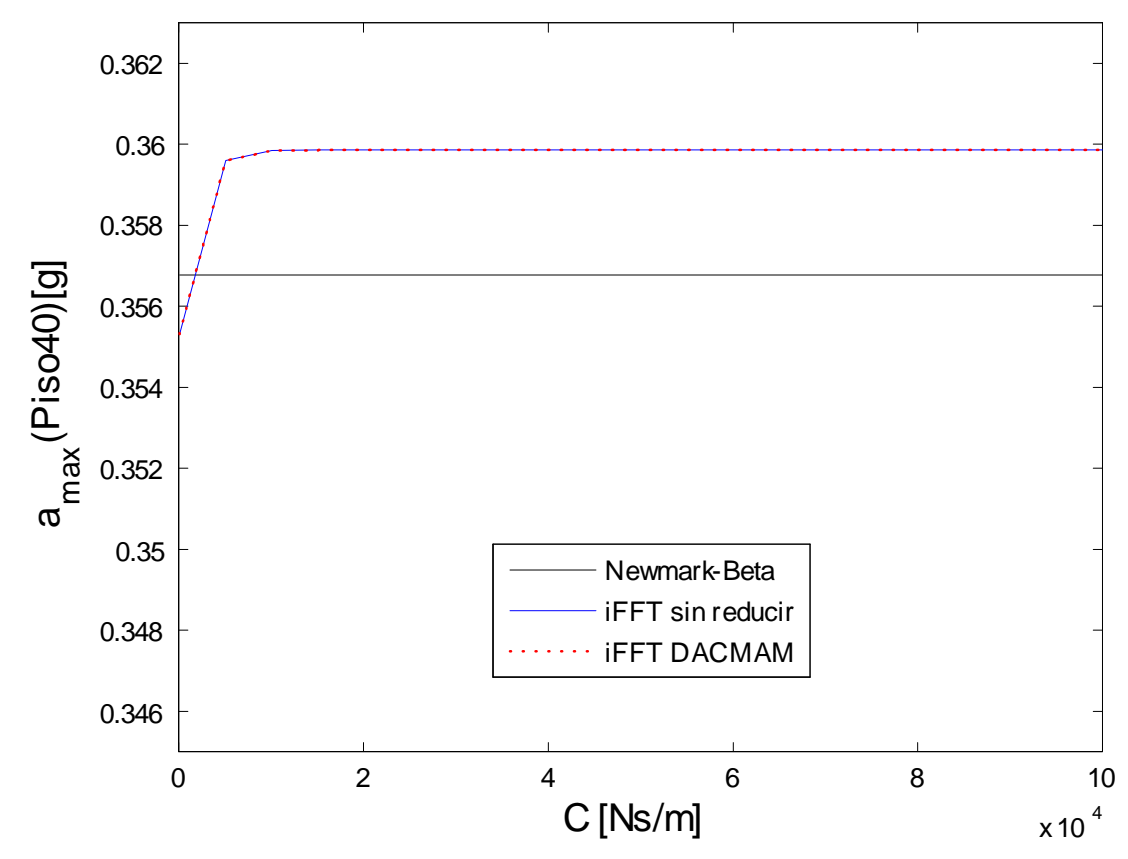

Figura 6-16: Respuesta máxima en aceleraciones de la planta 40 en función del parámetro de amortiguamiento del TMD. Comparación de métodos: algoritmo de integración Newmark-beta, inversa de Fourier con método DACMAM, e inversa de Fourier del modelo completo.

\begin{tabular}{|l|c|c|c|}
\cline { 2 - 4 } \multicolumn{1}{c|}{ tiempos [s] } & Portátil & Estación 2013 & Estación 2007 \\
\hline Espacio de los estados no simétrico, sin reducc. & 7086 & 5370 & 5194 \\
\hline SS I, sin reducción & 7063 & 5354 & 5181 \\
\hline Método DACMAM con truncatura a 5 modos & 1203 & 709 & 719 \\
\hline Método DACMAM sin truncatura & 1212 & 852 & 1012 \\
\hline DCSS I, caso A & 868 & 667 & 699 \\
\hline DCSS I, caso B (cond. iniciales diferentes) & 849 & 664 & 661 \\
\hline DCSS II, caso A & 886 & 664 & 661 \\
\hline DCSS III & 872 & 662 & 660 \\
\hline DCSS I, caso C (híbrido, con T del SS III) & 867 & 663 & 661 \\
\hline DCSS II, caso B (híbrido, con T del SS III) & 846 & 663 & 654 \\
\hline SCDS, (resuelto en SS I) & 845 & 663 & 641 \\
\hline SCSS I & 886 & 663 & 641 \\
\hline SCSS II & 868 & 667 & 640 \\
\hline
\end{tabular}

Tabla 6.30: Tiempos de computación de los diferentes métodos para el caso de parametrización del amortiguamiento del TMD en busca de minimizar aceleraciones. 


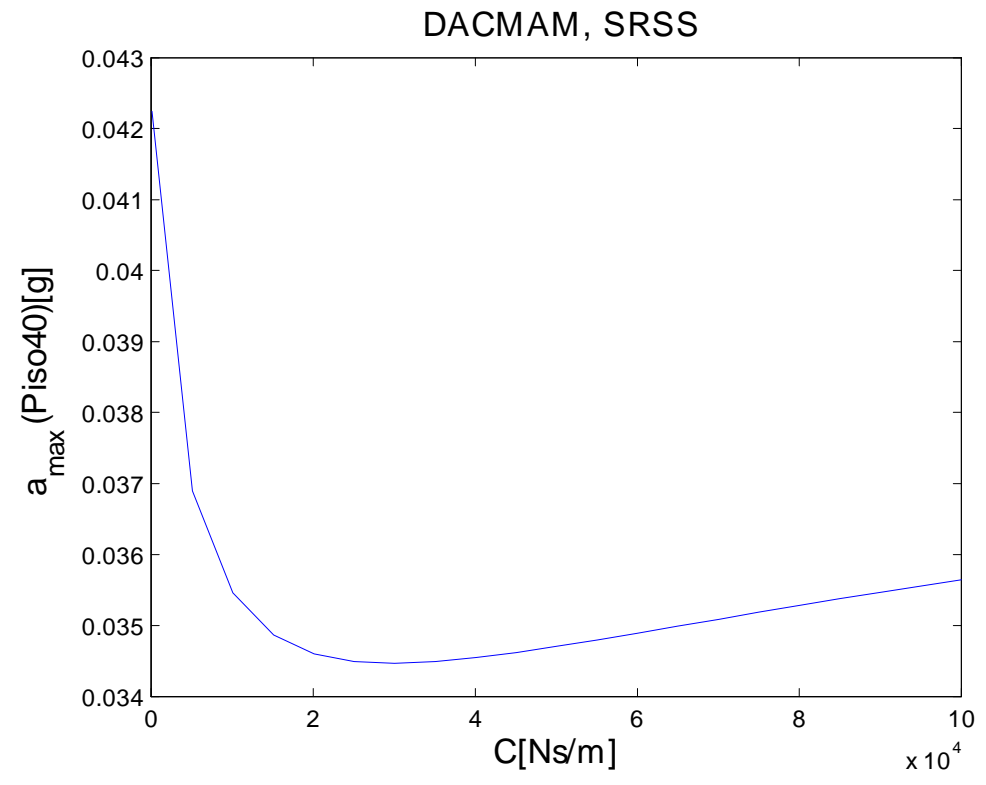

Figura 6-17: Respuesta máxima en aceleraciones de la planta 40 en función del parámetro de amortiguamiento del TMD. Método DACMAM, combinación SRSS.

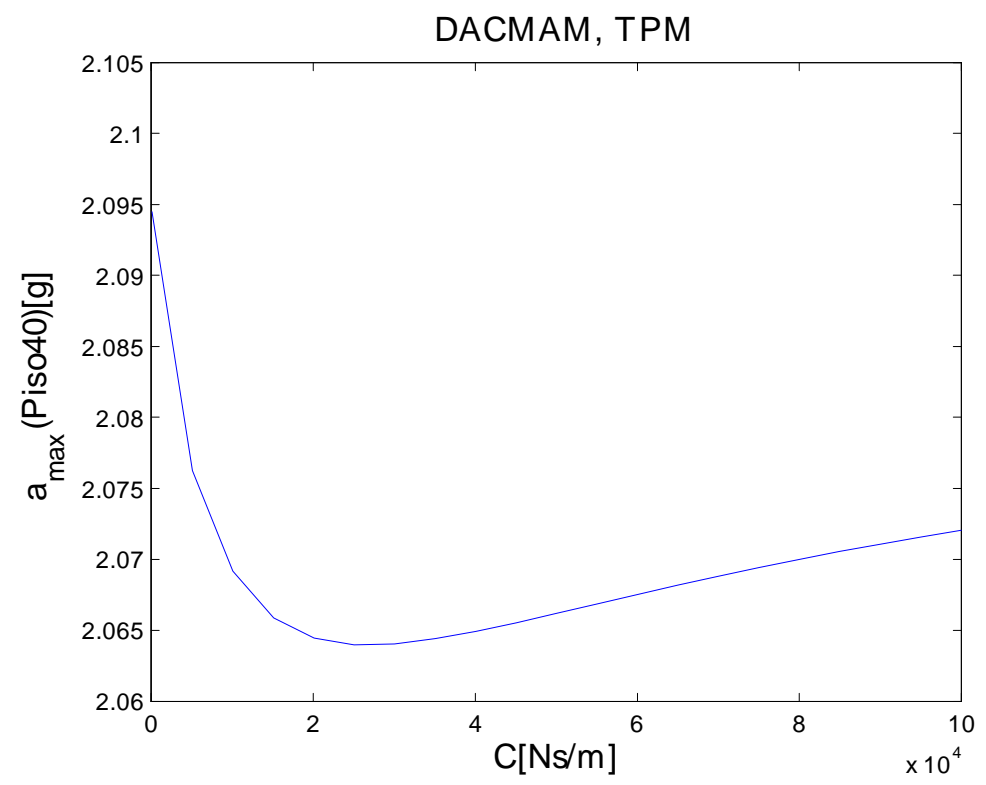

Figura 6-18: Respuesta máxima en aceleraciones de la planta 40 en función del parámetro de amortiguamiento del TMD. Método DACMAM, combinación TPM. 


\subsubsection{Parametrización de amortiguamiento del TMD para minimizar des- plazamientos}

Se ha considerado en este apartado una parametrización para la búsqueda del amortiguamiento del TMD que hace mínima la respuesta en desplazamientos en la última planta del edificio ante la carga del terremoto. Se parte igualmente de la respuesta permanente en frecuencias tomando como excitación las amplitudes dadas por la transformada discreta de Fourier del terremoto, que se ha refinado añadiendo ceros al final de la señal original para obtener un $\Delta f_{\text {análisis }}=0,0031 \mathrm{~Hz}$ (se pueden ver los tiempos de computación obtenidos en la tabla (6.32)).

Se muestran las respuestas de desplazamientos en frecuencia a la carga del terremoto con el problema completo y con el método DACMAM en la figura (6-19), para la planta 40, y para el valor óptimo de amortiguamiento.

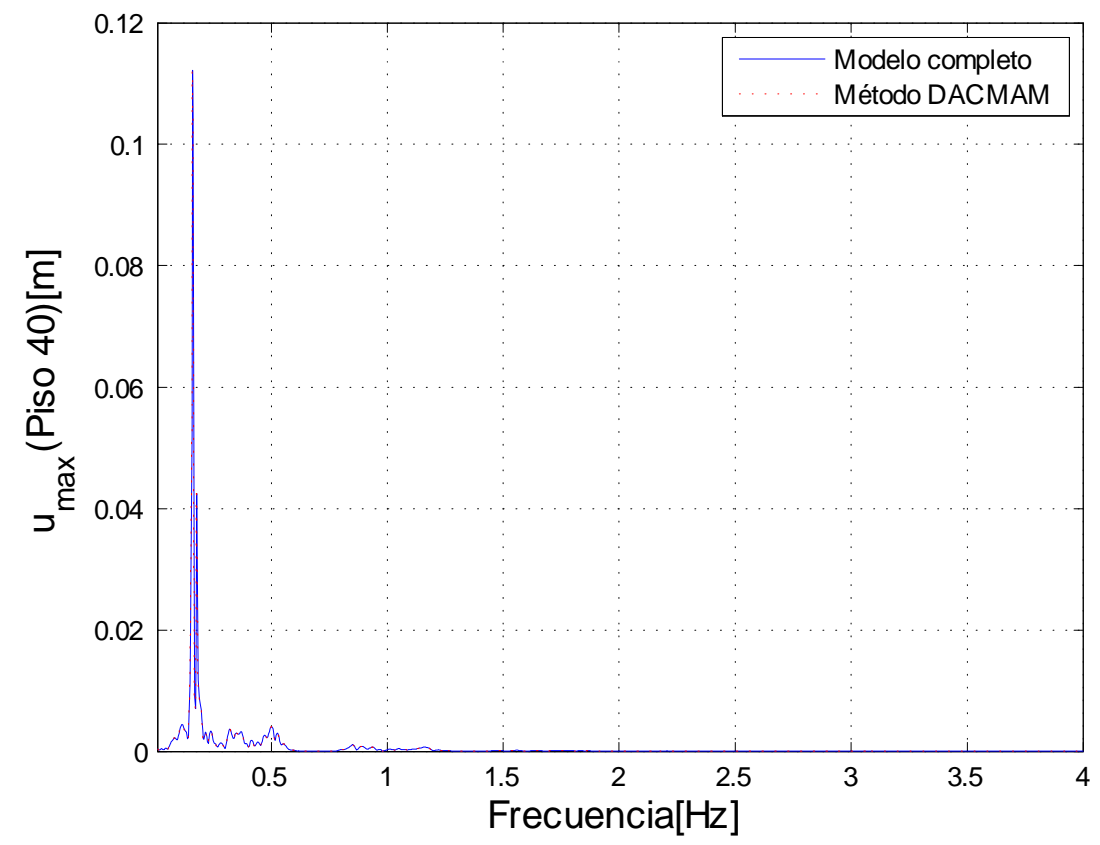

Figura 6-19: Resultados de desplazamiento de la planta 40, respuesta en frecuencia. Comparación de métodos: modelo completo en el espacio de los estados no simétrico y método DACMAM sin truncatura modal. 
En la figura (6-20) se muestra la respuesta temporal en desplazamientos calculada con el algoritmo implícito mencionado para el parámetro de amortiguamiento óptimo, y calculada empleando la transformada inversa de Fourier sobre la solución modal de respuesta permanente para el método $D A C M A M$, para el modelo sin reducción y para la condensación dinámica DCSSI, caso B (híbrido, con $T$ del SS III). Las diferencias entre los resultados se deben a la frecuencia de corte: y sobre todo a la densidad de puntos de la transformada discreta, ya que aumentando el número de puntos se pueden mejorar los resultados.

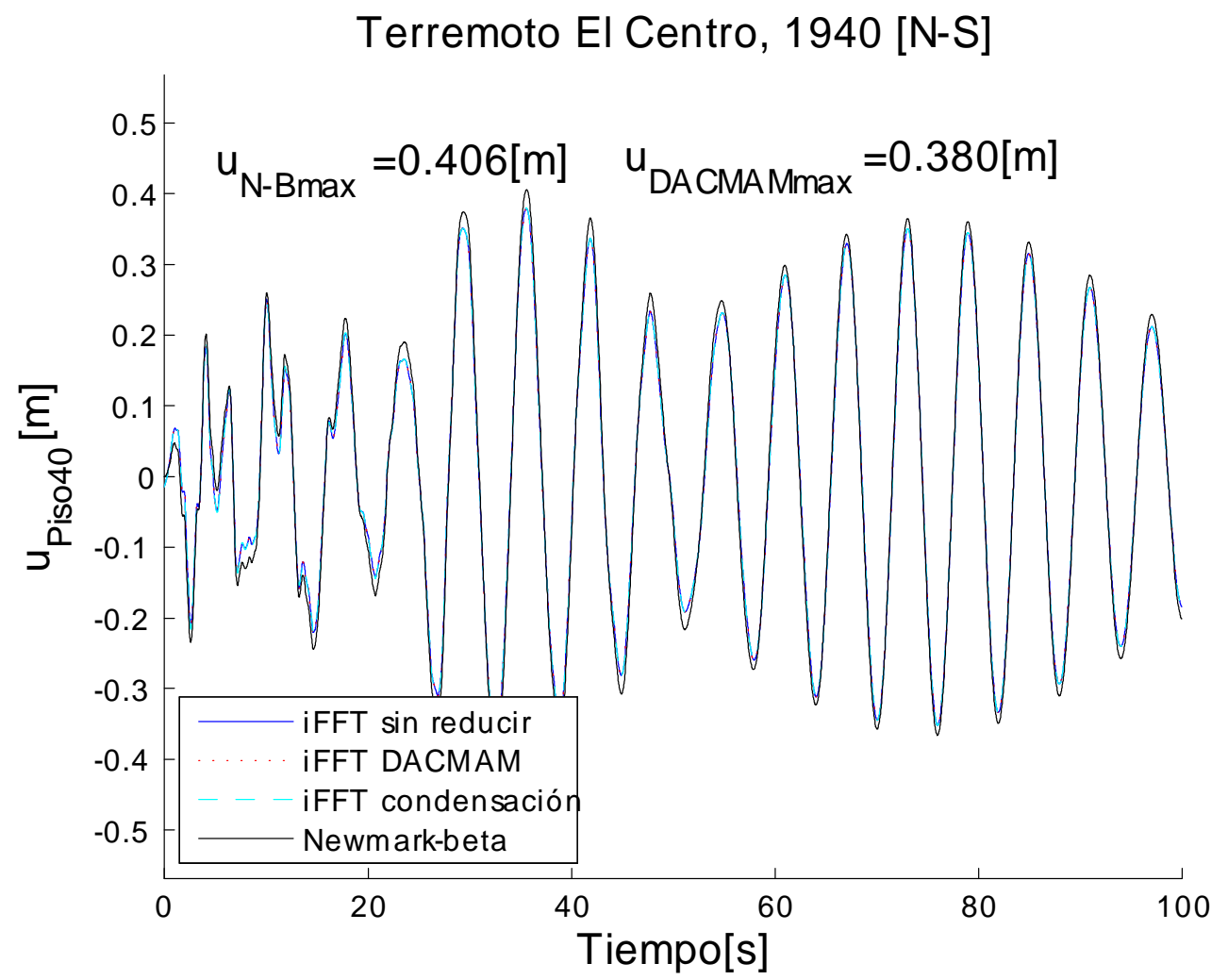

Figura 6-20: Respuesta temporal en desplazamientos de la planta 40 a la carga del terremoto. Caso óptimo de amortiguamiento del TMD para minimizar desplazamientos. Comparación de diversos métodos.

Se ha variado el amortiguamiento del TMD entre $\quad c_{T M D}=1 \cdot 10^{2} \mathrm{Ns} / \mathrm{m} \quad$ y $\quad c_{T M D}=1 \cdot 10^{5} \mathrm{Ns} / \mathrm{m}$. Se han tomado en dicho rango 21 valores equiespaciados. En la figura (6-21) se muestra cómo en el rango seleccionado se encuentra un mínimo de la respuesta buscada, $c_{T M D}=5095 \mathrm{Ns} / \mathrm{m}$, que es un $6 \%$ del valor inicial del ejemplo de estudio. En dicha figura se muestran los resultados 
de diferentes métodos: integración numérica con el métodos Newmark-beta y a partir de la transformada inversa de Fourier aplicada sobre los resultados del modelo completo, del método $D A C M A M$ y de la condensación DCSS II, caso B. Se muestra también para comparar, en las figuras (6-22 y 6-23), la misma gráfica para el método DACMAM con las combinaciones SRSS y TPM respectivamente, con truncatura modal empleando únicamente la información de los 5 primeros modos.

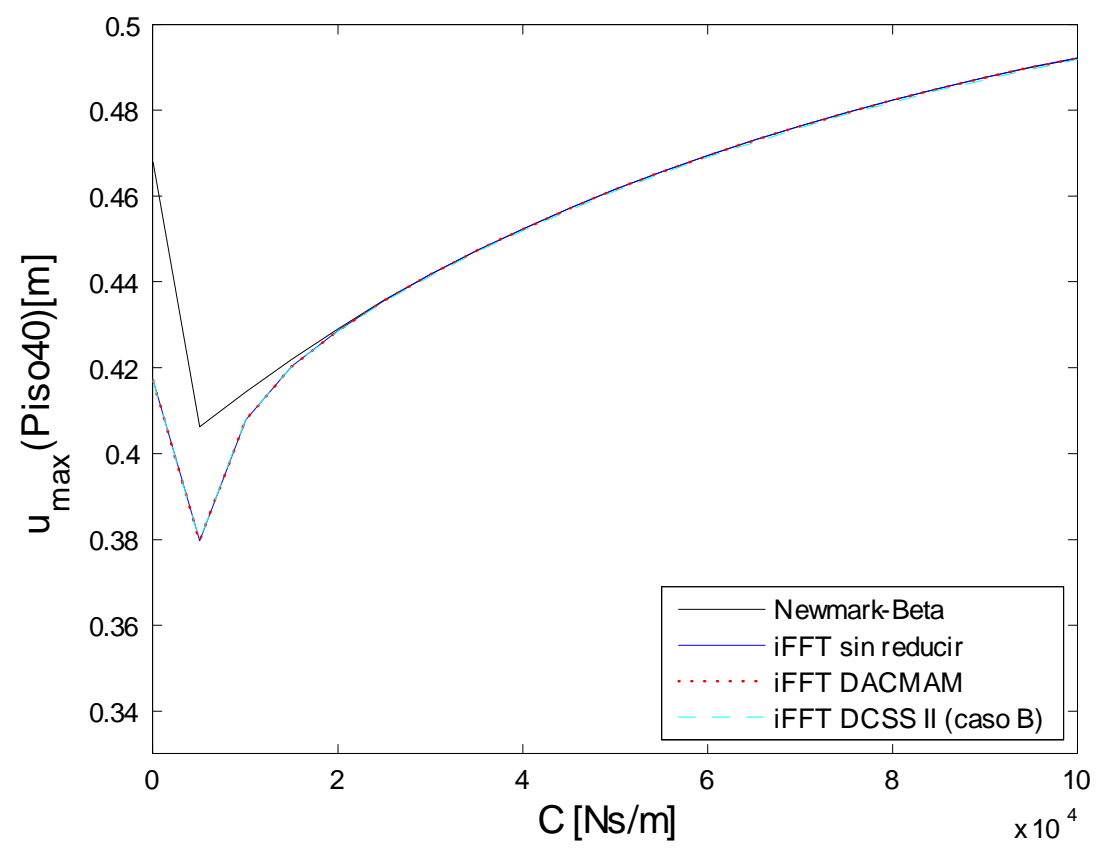

Figura 6-21: Respuesta máxima en desplazamientos de la planta 40 en función del parámetro de amortiguamiento del TMD. Comparación de métodos: algoritmo de integración Newmark-beta, e inversas de Fourier del método DACMAM, del modelo sin reducir y de la condensación DCSS II, caso B (híbrido, con T del SS III).

Como se ha comentado, las diferencias entre la transformada inversa de Fourier y el método de integración Newmark-beta se deben a dos motivos: por un lado, con valores bajos de amortiguamiento el error por no considerar la respuesta transitoria en la solución modal es mayor, y por otro lado la respuesta en frecuencias está truncada con una frecuencia de corte de $f_{\text {máx }}=20 H z$ y la transformada, al ser discreta, introduce también un error dependiente de la densidad de puntos $\left(\Delta f_{\text {análisis }}\right)$. Este segundo motivo del error sucede sobre todo para amor- 


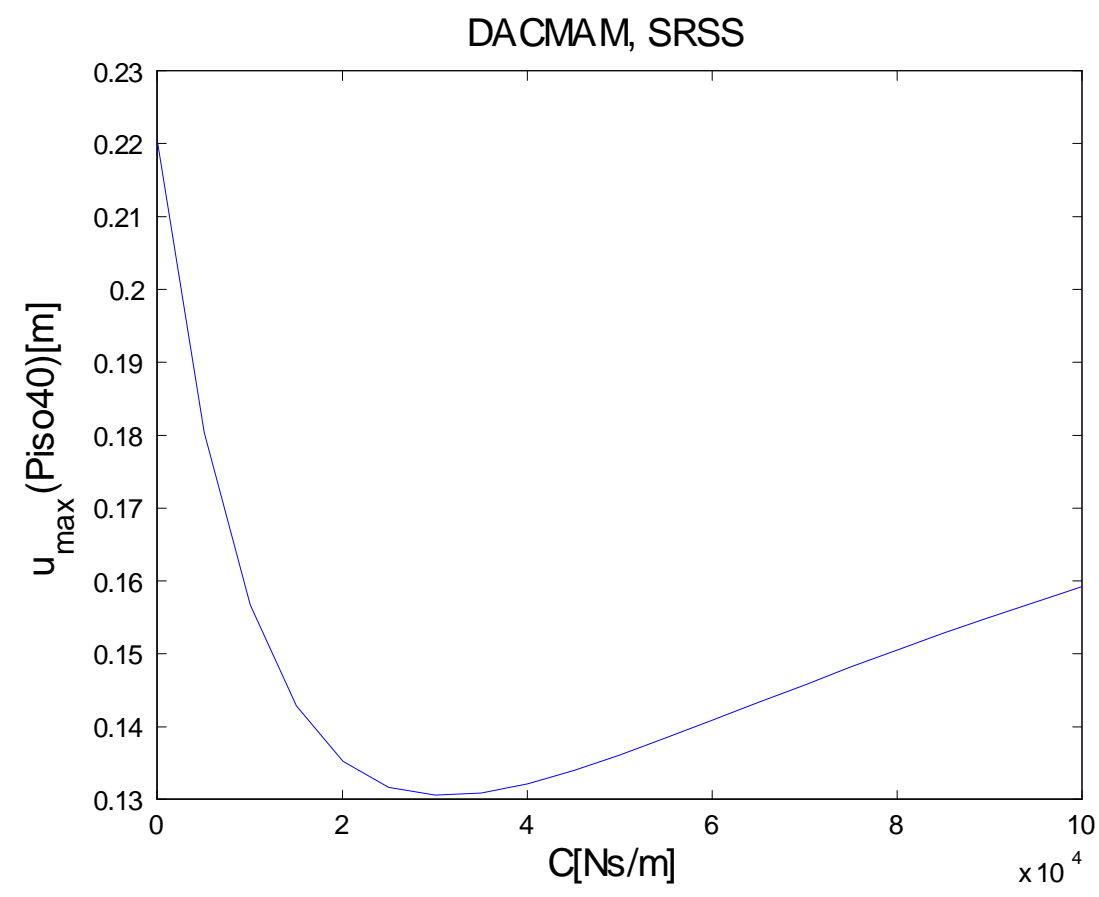

Figura 6-22: Respuesta máxima en desplazamientos de la planta 40 en función del parámetro de amortiguamiento del TMD. Método DACMAM, combinación SRSS.

tiguamientos pequeños, donde los picos en frecuencia son más estrechos y por tanto requieren de una densidad de frecuencias muy fina al evaluar la respuesta en frecuencias de la estructura. A pesar de las diferencias, se puede observar que el valor óptimo se obtiene de modo exacto dentro de los 21 valores considerados.

En la tabla (6.31) se muestran los valores óptimos del parámetro $c_{T M D}$ en función del método empleado con las herramientas mencionadas anteriormente. Los métodos de reducción han tomado sólo 5 nodos maestros, correspondientes a los grados de libertad de las plantas 10, 20, 30, 40 y al TMD.

En esta parametrización se puede observar que los resultados de las combinaciones espectrales no son tan buenos como en el caso de la rigidez, siendo el método TPM el que ofrece mejores resultados en este caso como herramienta cualitativa para la búsqueda del amortiguamiento óptimo.

Para una correcta interpretación de los resultados se debe tener en cuenta que el rango de 


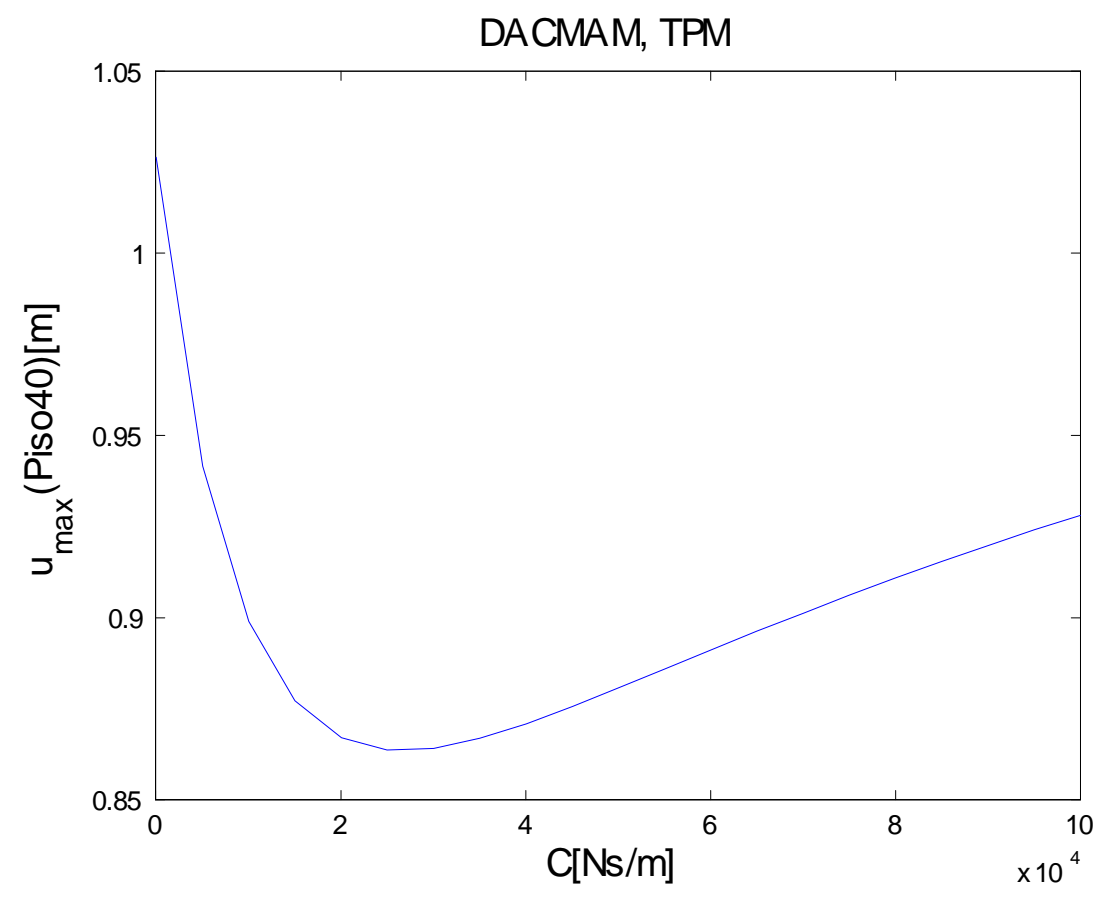

Figura 6-23: Respuesta máxima en desplazamientos de la planta 40 en función del parámetro de amortiguamiento del TMD. Método DACMAM, combinación TPM.

variación de la respuesta supervisada es pequeño en el rango de variación del parámetro de amortiguamiento. Estos datos se pueden ver en la figura (6-21). Se indican aquí adicionalmente unos valores de interés:

$$
\begin{array}{cc}
u_{\text {opt }}(c=5095 \mathrm{Ns} / \mathrm{m})=0,406 \mathrm{~m} & u(c=25075 \mathrm{Ns} / \mathrm{m})=0,436 \mathrm{~m} \\
u\left(c_{\text {mín }}=100 \mathrm{Ns} / \mathrm{m}\right)=0,470 \mathrm{~m} & u\left(c_{\text {máx }}=10^{5} \mathrm{Ns} / \mathrm{m}\right)=0,490 \mathrm{~m}
\end{array}
$$

Se observa que el error de las soluciones proporcionadas por los diversos métodos es aceptable exceptuando el método de condensación estática I.

En la tabla (6.32) se muestran los tiempos requeridos en función del método para esta parametrización:

Se observa que el método DACMAM (sin tener en cuenta el resultado del portátil, del que se hablará más adelante) proporciona tiempos de computación del mismo orden que las 


\begin{tabular}{|l|c|c|c|c|c|}
\multicolumn{1}{|c|}{$c_{\text {TMD }}^{\text {ótimo }}[\mathrm{Ns} / \mathrm{m}]$} & iFFT & MAX & ASM & SRSS & TPM \\
\hline Espacio de los estados no simétrico, sin reducc. & 5095 & 50050 & 25075 & 30070 & 25075 \\
\hline SS I, sin reducción & 5095 & 50050 & 25075 & 30070 & 25075 \\
\hline Método DACMAM con truncatura a 5 modos & 5095 & 50050 & 25075 & 30070 & 25075 \\
\hline Método DACMAM sin truncatura & 5095 & 50050 & 25075 & 30070 & 25075 \\
\hline DCSS I, caso A & 5095 & 50050 & 25075 & 30070 & 25075 \\
\hline DCSS I, caso B (cond. iniciales diferentes) & 5095 & 50050 & 25075 & 30070 & 25075 \\
\hline DCSS II, caso A & 5095 & 50050 & 25075 & 30070 & 25075 \\
\hline DCSS III & 5095 & 50050 & 25075 & 30070 & 25075 \\
\hline DCSS I, caso C (híbrido, con T del SS III) & 5095 & 50050 & 25075 & 30070 & 25075 \\
\hline DCSS II, caso B (híbrido, con T del SS III) & 5095 & 50050 & 25075 & 30070 & 25075 \\
\hline SCDS, (resuelto en SS I) & 5095 & 45055 & 25075 & 30070 & 25075 \\
\hline SCSS I & 100000 & 100000 & 100000 & 100000 & 100000 \\
\hline SCSS II & 5095 & 45055 & 25075 & 30070 & 25075 \\
\hline Algoritmo de integración Newmark-beta & 5095 & n/a & n/a & n/a & n/a \\
\hline
\end{tabular}

Tabla 6.31: Valores óptimos de amortiguamiento del TMD para minimizar la respuesta en desplazamientos ante la carga del terremoto.

condensaciones, pero proporcionando resultados con la información del modelo completo. De acuerdo a lo mostrado en apartados anteriores, al aumentar el número de nodos maestros, lo cual es inevitable para modelos de gran tamaño, los tiempos de proceso de los métodos de condensación aumentarán superando el tiempo de proceso muy por encima del requerido por el método DACMAM.

El tiempo proporcionado por el método DACMAM sin truncatura en el equipo portátil es anormalmente alto si se compara con los resultados que se obtienen en los otros ejemplos. Esto se debe a que se realiza la computación de los tiempos de todos los métodos a la vez, por lo que se ha requerido más memoria de acceso aleatorio que la disponible en el equipo. Aunque el modelo tiene un tamaño muy reducido, se almacenan durante el proceso un elevado número de variables, mucho mayor que las necesarias para obtener la solución, con el fin de controlar todos los aspectos del problema. Esto ha dado lugar a la alta demanda de memoria, y tras solicitar toda la memoria de acceso aleatorio disponible, se emplea memoria del disco duro, mucho más lenta. Por otro lado, este problema afecta de modo más importante al método $D A C M A M$ puesto que hace un uso más intensivo de los datos almacenados que el resto de métodos. Para corroborar que éste era el motivo, se ha repetido el problema de modo idéntico con la sola excepción de resolver únicamente el problema por medio del método DACMAM sin 


\begin{tabular}{|l|c|c|c|}
\cline { 2 - 4 } \multicolumn{1}{c|}{ tiempos [s] } & Portátil & Estación 2013 & Estación 2007 \\
\hline Espacio de los estados no simétrico, sin reducc. & 2533 & 2167 & 2133 \\
\hline SS I, sin reducción & 2540 & 2181 & 2133 \\
\hline Método DACMAM con truncatura a 5 modos & 369 & 275 & 284 \\
\hline Método DACMAM sin truncatura & 1332 & 366 & 460 \\
\hline DCSS I, caso A & 325 & 275 & 320 \\
\hline DCSS I, caso B (cond. iniciales diferentes) & 320 & 275 & 275 \\
\hline DCSS II, caso A & 319 & 275 & 277 \\
\hline DCSS III & 320 & 275 & 276 \\
\hline DCSS I, caso C (híbrido, con T del SS III) & 319 & 276 & 277 \\
\hline DCSS II, caso B (híbrido, con T del SS III) & 319 & 275 & 273 \\
\hline SCDS, (resuelto en SS I) & 314 & 276 & 260 \\
\hline SCSS I & 312 & 274 & 260 \\
\hline SCSS II & 313 & 271 & 260 \\
\hline
\end{tabular}

Tabla 6.32: Tiempos de computación de los diferentes métodos para el caso de parametrización del amortiguamiento del TMD en busca de minimizar desplazamiento.

truncatura, dando lugar a un tiempo de proceso de 496s. 


\subsubsection{Parametrización de masas del edificio. Búsqueda de respuesta máxima}

En este apartado se describe una parametrización de masas del edificio (figura 6-24) simulando la masa no estructural que puede variar en los mismos por diversos motivos: nivel de ocupación de personas, mobiliario, la instalación de elementos con una gran masa concentrada (una piscina de recreo, depósito de agua antiincendios, almacenes de diferente tipo, etc.). En este caso no se busca una combinación de parámetros óptima, sino validar diferentes configuraciones másicas aplicadas sobre la estructura para determinar limitaciones en su uso que puedan afectar a la seguridad. Se considera una limitación en el desplazamiento máximo que puede aparecer en la planta 40 del edificio durante la carga del terremoto, por lo que se supervisa su valor máximo.

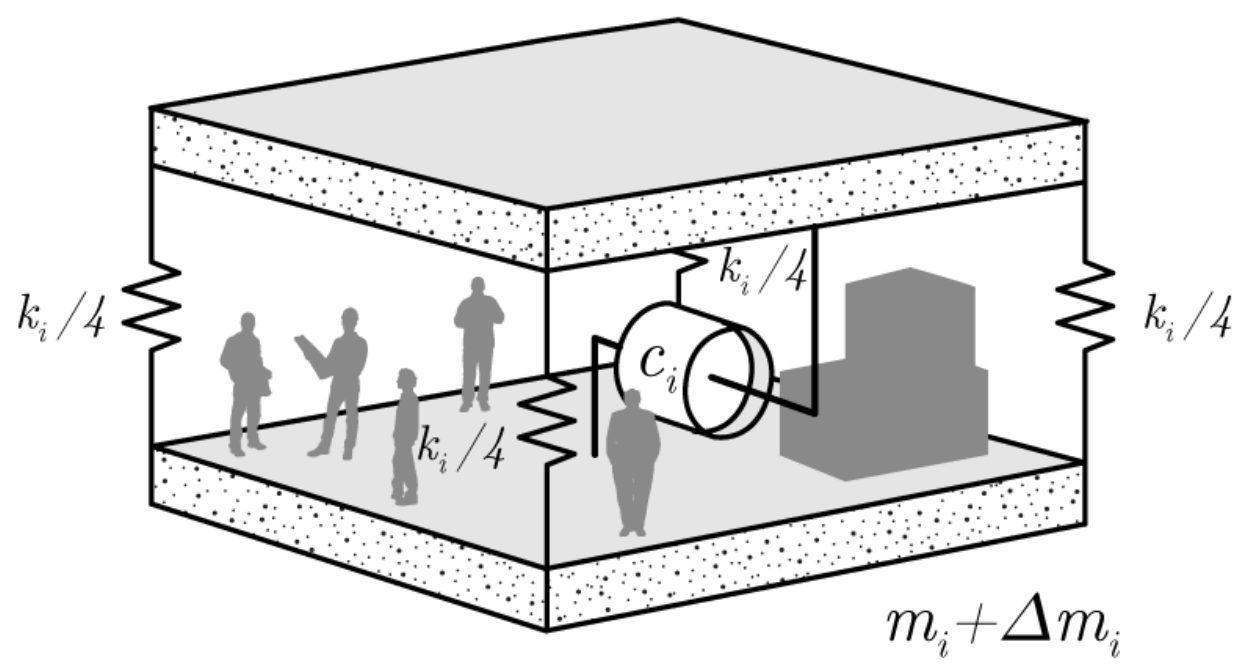

Figura 6-24: Esquema de la parametrización de masas en una planta del edificio.

Para el ejemplo en cuestión se considerarán 3 niveles de masa añadida en las 5 plantas superiores, que dan lugar a $3^{5}=243$ casos de análisis. No se contempla una variación de la masa de todas las plantas para hacer el problema abordable en un tiempo razonable y por escapar de las necesidades de comparación de la tesis. 
Se ha considerado el mismo rango de variación de masa adicional en cada planta: un $0 \%$, un $30 \%$ y un $80 \%$ de la masa original de la planta. Esta variación se puede plantear a medida para cada planta sin por ello modificar en ningún aspecto el esfuerzo de computación y de modo muy sencillo en el caso del método DACMAM. Para los métodos de condensación tradicionales dicha consideración no es tan sencilla y la parametrización implica tener la capacidad de poder automatizar la creación de modelos paramétricos específicos para el software comercial de elementos finitos que se emplee, lo que pone de relieve la gran versatilidad del método DACMAM.

En la figura (6-25) se puede ver la variación de la respuesta en desplazamientos para los parámetros considerados con varios métodos. En el eje de abscisas se indica el grupo de parámetros considerados (de 1 a 243, que son el total de parametrizaciones).

Como se puede ver, los resultados obtenidos a partir de la transformada inversa de Fourier proporcionan una aproximación muy buena, ya que se ha evaluado la respuesta a partir de la información modal de la respuesta permanente (la respuesta transitoria tiene poco peso en este problema y el error se debe al hecho de emplear transformadas discretas y que la señal del terremoto no es suave), calculada tomando como entrada los datos de la transformada discreta de Fourier de la carga del terremoto (figura 5-7). Los resultados de la respuesta con el método de condensación DCSS II, caso B (híbrido, con T del SS III), que es de todas las condensaciones la que mejores resultados modales mostraba, es el método que peores resultados proporciona de los mostrados en la figura.

En la tabla (6.33) se pueden ver los tiempos de análisis que requieren los diferentes métodos considerados (las condensaciones se han realizado con 10 gdl retenidos: plantas 5, 10, 15, 20, $25,30,34,38,40$ y el gdl correspondiente al TMD) pero sin tener en cuenta el tiempo de la transformada inversa de Fourier.

Para este caso, los métodos de reducción iterativos, especialmente los que emplean la matriz de transformación de coordenadas $T^{I I I}$, proporcionan unos resultados con un error muy pequeño y en tiempos muy reducidos. Esto se debe al pequeño tamaño del problema En modelos grandes, donde el número de grados de libertad es varios órdenes de magnitud superior, y donde en general se tiene un número de nodos maestros mayor, los tiempos que requieren los métodos de condensación serán mayores que el método DACMAM al ser necesario en general retener 


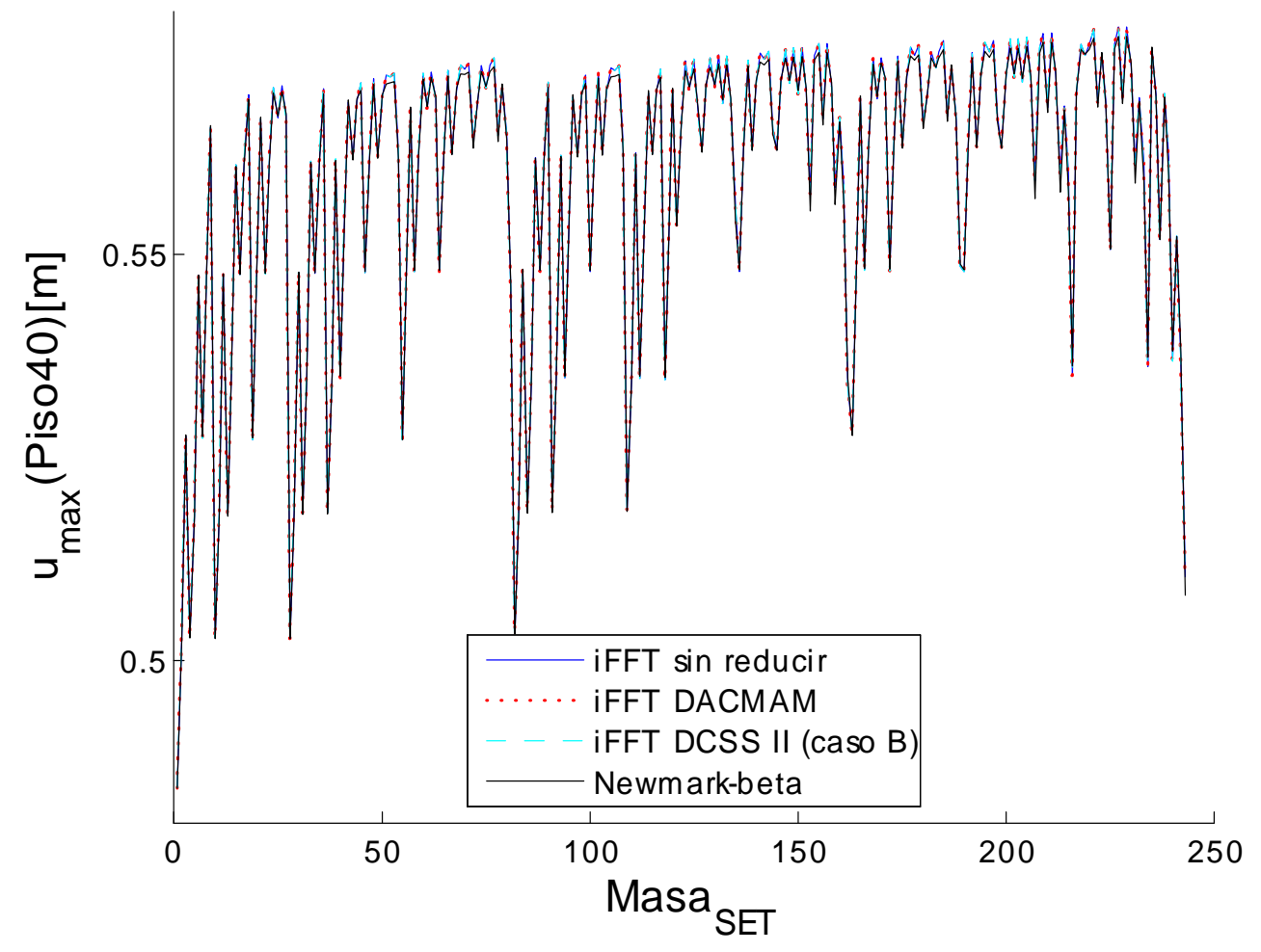

Figura 6-25: Respuesta máxima en desplazamientos de la planta 40 en función del grupo de masas adicionales. Comparación de métodos: algoritmo de integración Newmark-beta, e inversas de Fourier del método DACMAM, del modelo sin reducir y de la condensación DCSS II, caso $B$ (híbrido, con T del SS III).

más nodos para simular mejor la respuesta dinámica del sistema. En el caso de estructuras con una concentración de modos en el rango de frecuencias de interés los ahorros de tiempo que proporciona el método $D A C M A M$ son aún más importantes por la exigencia de retener más modos en el problema reducido. 


\begin{tabular}{|l|c|c|c|}
\cline { 2 - 4 } \multicolumn{1}{c|}{ tiempos [s] } & Portátil & Estación 2013 & Estación 2007 \\
\hline Espacio de los estados no simétrico, sin reducc. & 11335 & 7878 & 8497 \\
\hline SS I, sin reducción & 11300 & 7863 & 8435 \\
\hline Método DACMAM sin truncatura & 4040 & 2636 & 3750 \\
\hline DCSS I, caso A & 2666 & 1949 & 2640 \\
\hline DCSS I, caso B (cond. iniciales diferentes) & 2555 & 1948 & 2127 \\
\hline DCSS II, caso A & 2537 & 1950 & 2184 \\
\hline DCSS III & 2537 & 1949 & 2152 \\
\hline DCSS I, caso C (híbrido, con T del SS III) & 2553 & 1949 & 2153 \\
\hline DCSS II, caso B (híbrido, con T del SS III) & 2510 & 1950 & 2154 \\
\hline SCDS, (resuelto en SS I) & 2461 & 1961 & 1960 \\
\hline SCSS I & 2469 & 1931 & 1961 \\
\hline SCSS II & 2446 & 1931 & 1961 \\
\hline
\end{tabular}

Tabla 6.33: Tiempos de computación de los diferentes métodos para el caso de parametrización de masas adicionales en las últimas plantas del edificio.

\subsubsection{Parametrización de rigideces del edificio. Búsqueda de respuesta má- xima}

Se ha considerado también paramétricamente el efecto en el edificio de arriostramientos tipo cruz de San Andrés, instalados en distintas plantas (figura 6-26). La modificación sobre la rigidez de la planta a carga transversal que dichos refuerzos implican se puede aproximar, a partir de las propiedades físicas del material de las vigas que conforman la cruz y la dimensión según $x$ del edificio (ancho), por $\Delta k=\frac{4 E_{\text {acero }} A_{\text {refuerzo }}}{L_{\text {viga }}}$. Debido al carácter académico del ejemplo, se ha considerado la hipótesis simplificadora de que los arriostramientos contribuyen a la rigidez únicamente cuando están sometidos a tracción (hipótesis coherente con el hecho de que, en una estructura real de en un edificio, la configuración seleccionada daría lugar a vigas muy esbeltas), y por la simetría se puede considerar que son dos los refuerzos que contribuyen con una rigidez igual en ambos sentidos de la dirección $x$, el único grado de libertad considerado en el ejemplo. Los refuerzos se consideran de acero y el ancho del edificio por planta constante de longitud 80 metros: $E_{\text {acero }}=200 G P a$, y $L_{\text {refuerzo }}=80 \mathrm{~m}$.

Se consideran como plantas susceptibles de refuerzo las plantas pares inferiores: 2, 4, 6, 8, 10 y 12, y como refuerzo se consideran 3 supuestos: sin refuerzo, con refuerzo de arriostramientos de sección constante $A_{\text {refuerzo }}=20 \mathrm{~cm}^{2}$ o de sección $2 A_{\text {refuerzo }}$.

Se ha realizado un ejercicio análogo al del apartado anterior, pero buscando las respuestas 


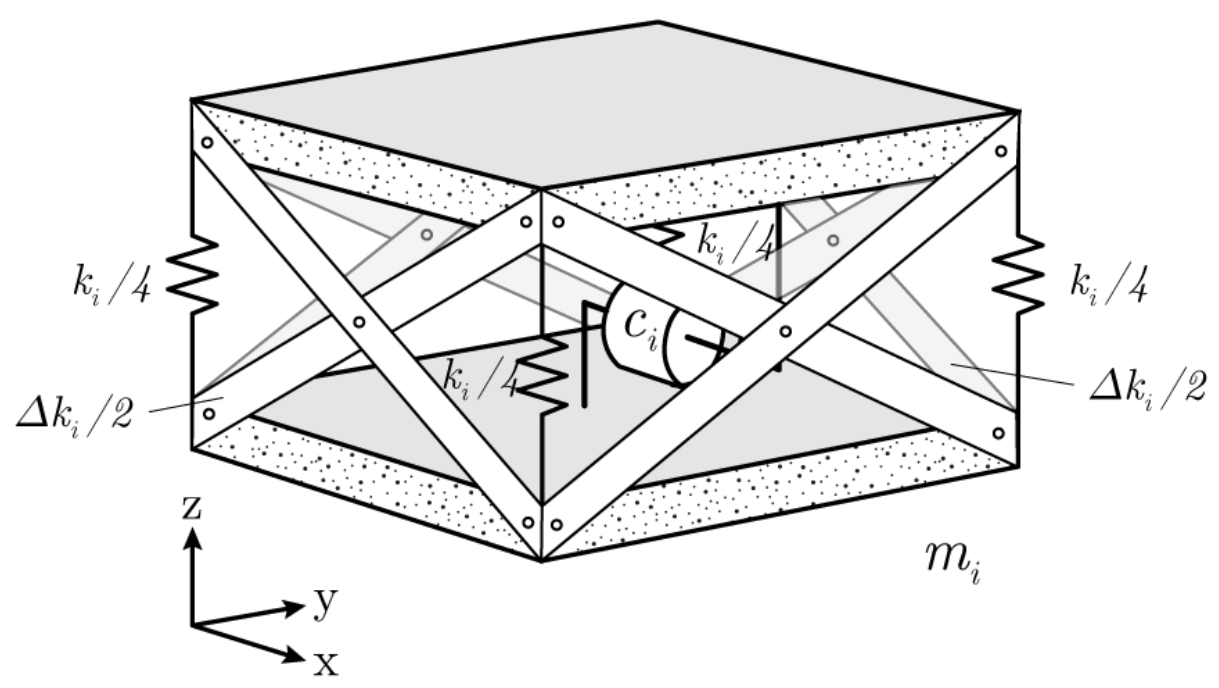

Figura 6-26: Esquema de la parametrización de rigideces en una planta del edificio.

máximas en aceleraciones con las diferentes configuraciones.

En la figura (6-27) se puede ver la variación de la respuesta en aceleraciones para los parámetros considerados obtenida por integración numérica con el método Newmark-beta. En el eje de abscisas se indica el grupo de parámetros considerados (de 1 a 729, que son el total de parametrizaciones).

También se muestra la respuesta del método DACMAM con toda la información de los modos en la figura (6-28). Como se puede ver, los resultados tienen una forma idéntica y sólo se diferencian en el valor (un $4 \%$ de error que se debe a la frecuencia de corte elegida, $f_{\text {máx }}=12 \mathrm{~Hz}$, y la baja densidad de puntos en el dominio de la frecuencia, $\left.\Delta f_{\text {análisis }}=0,0061 \mathrm{~Hz}\right)$. En este caso se ha evaluado la respuesta a partir de la información modal para la respuesta permanente (la parte transitoria se ha obviado en este problema), que se obtiene a su vez de los datos de la transformada discreta de Fourier de la carga del terremoto.

Se muestra asimismo una última figura (6-29) en la que se comparan los resultados obtenidos a partir de la transformada inversa de Fourier, y en la que se observa que el método DACMAM y el modelo completo proporcionan los mismos resultados mientras que la condensación dinámica presenta errores apreciables. 


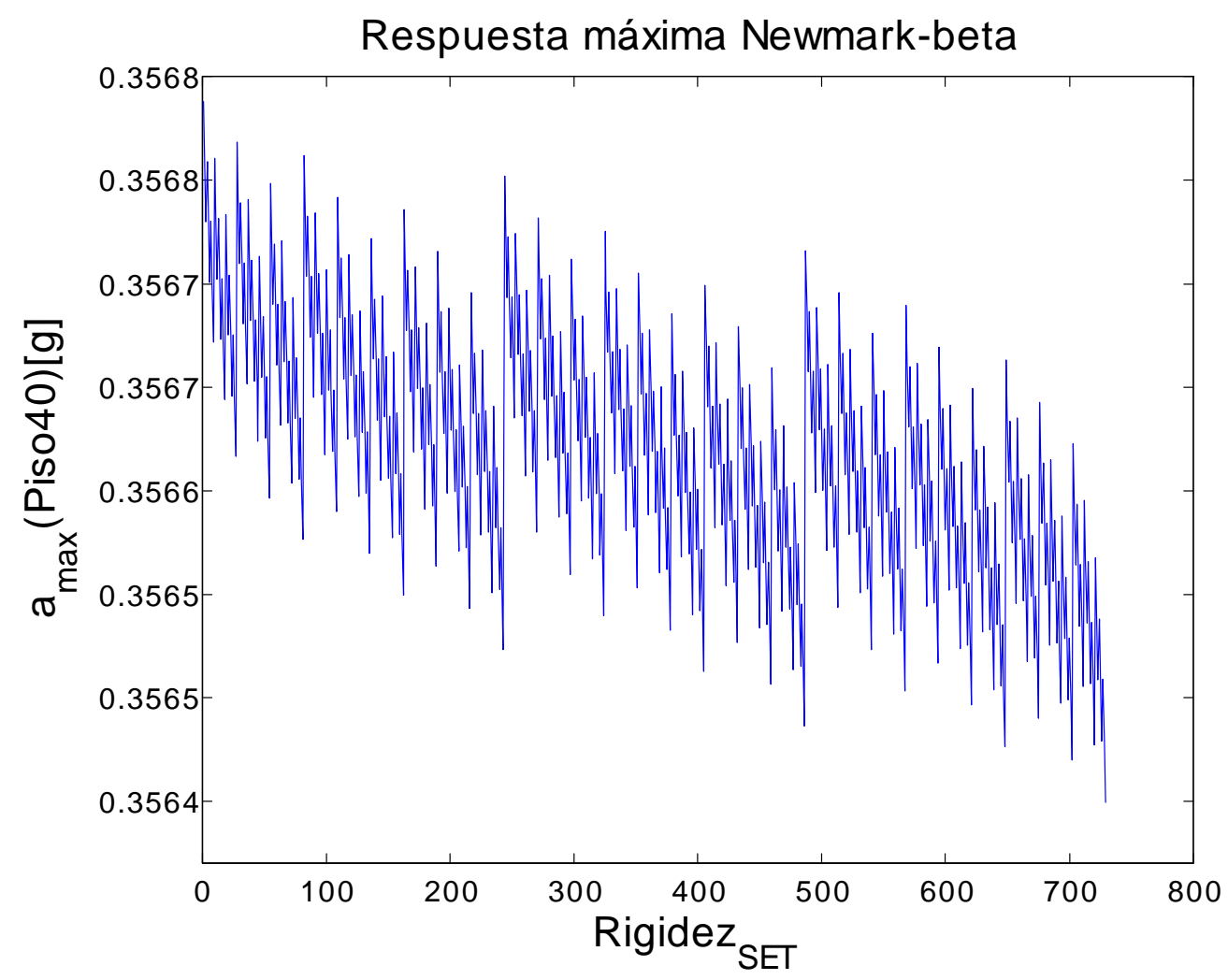

Figura 6-27: Respuesta máxima en aceleraciones de la planta 40 en función de grupo de rigideces adicionales consideradas. Algoritmo de integración Newmark-beta.

En la tabla (6.34) se pueden ver los tiempos de análisis que requieren los diferentes métodos considerados, teniendo las condensaciones los mismos 10 nodos maestros que en la parametrización de masas, e igual que en dicho caso sin tener en cuenta el tiempo de la transformada inversa de Fourier.

Respecto a la calidad de los resultados se repite lo descrito en los apartados anteriores, pero en este caso, debido al mayor número de las parametrizaciones, se pone más de relieve el potencial ahorro en tiempo que proporciona el método DACMAM.

Como se observa en las figuras de las respuestas del sistema, se tiene toda la información necesaria en los puntos de interés para determinar el efecto en la respuesta dinámica de la estructura ante estas modificaciones. Aunque no se tratarán en más profundidad los resultados, cabe destacar que esta parametrización se podría unir a la anterior de masas en una segunda 


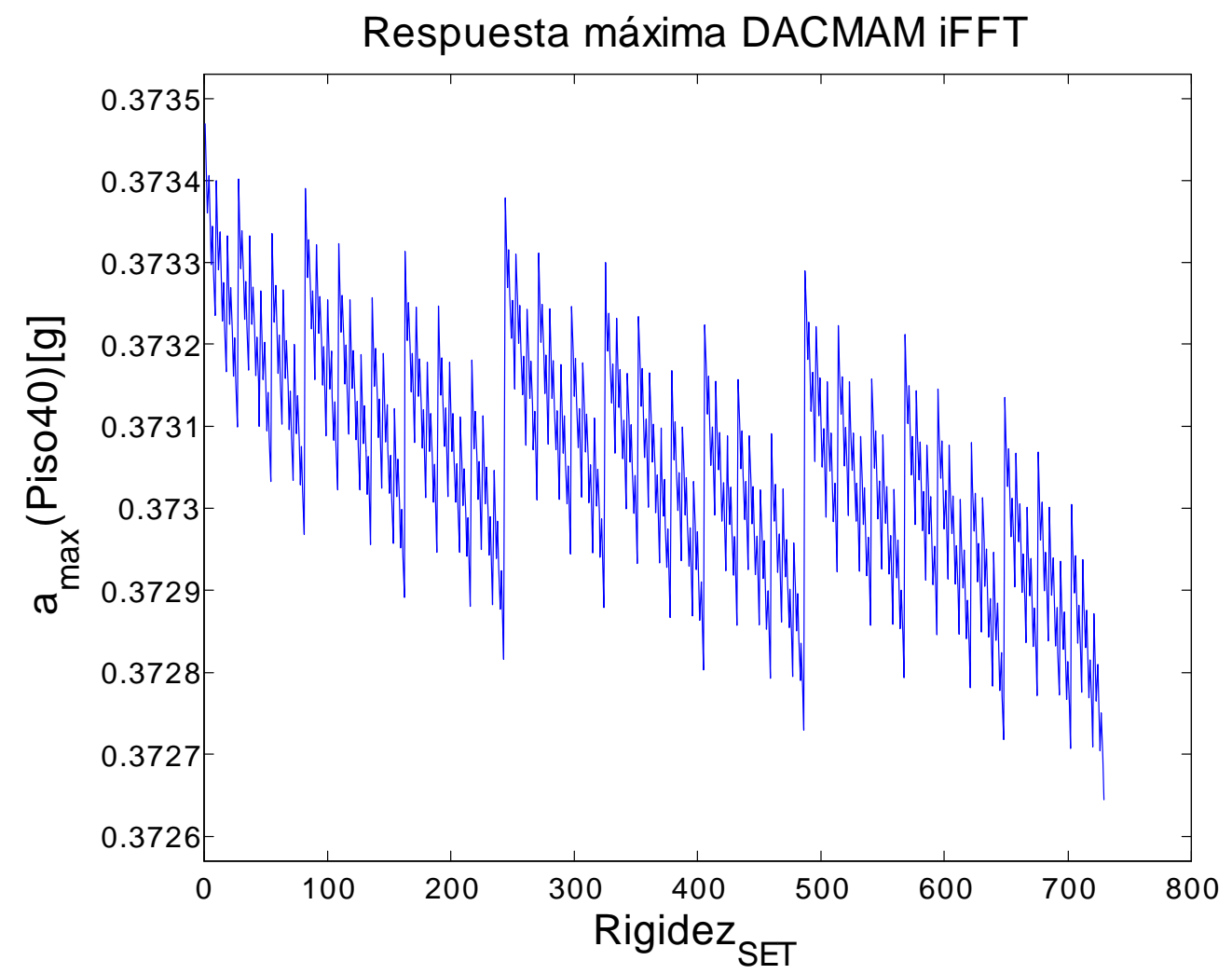

Figura 6-28: Respuesta máxima en aceleraciones de la planta 40 en función de grupo de rigideces adicionales consideradas. Método DACMAM.

fase con el fin de aceptar configuraciones másicas descartadas porque producían una elevada respuesta, de modo que se obtuviera una respuesta dinámica dentro del rango aceptable con la adición de refuerzos, o la instalación de TMD adicionales si fueran necesarios. 


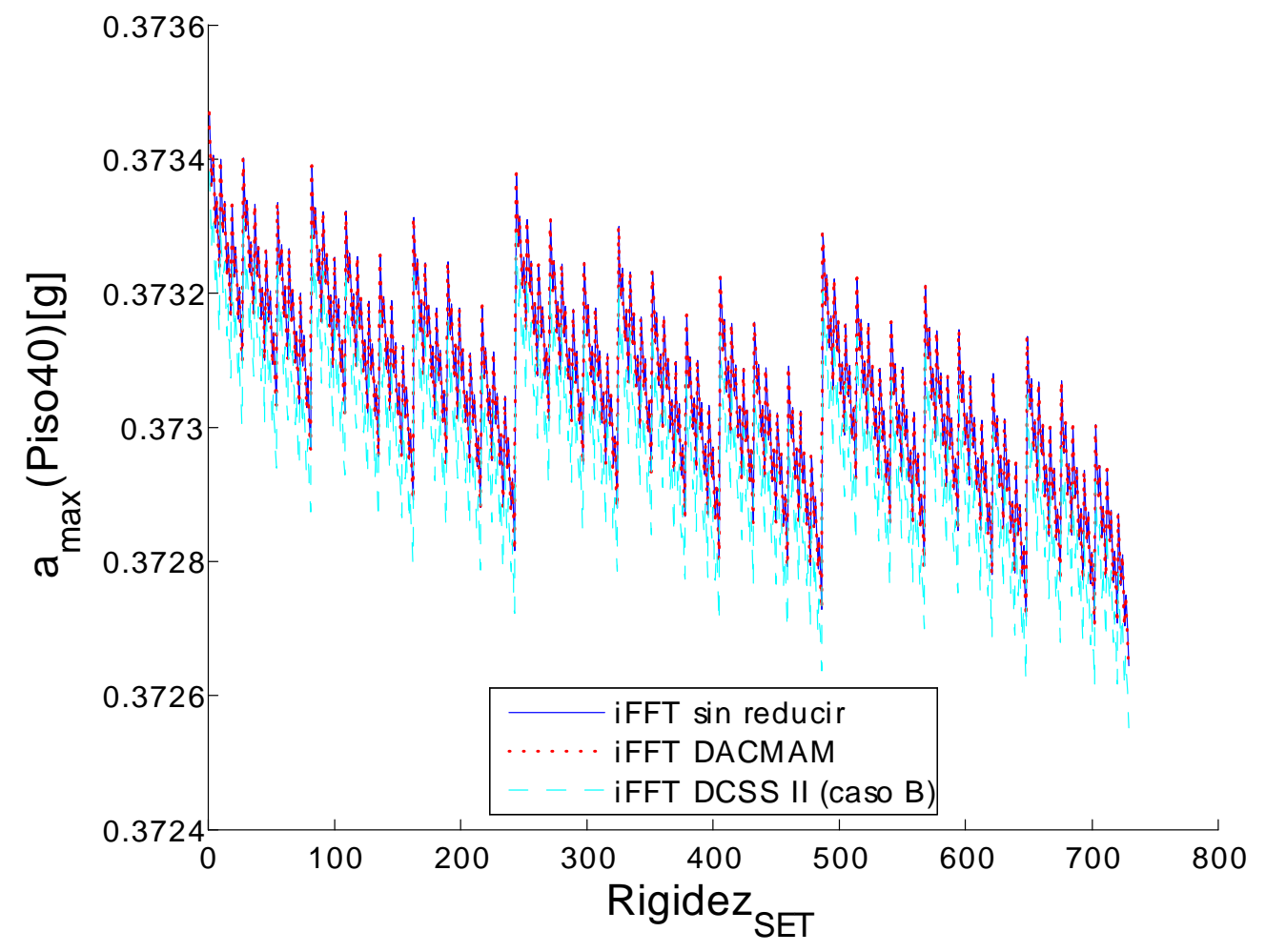

Figura 6-29: Respuesta máxima en aceleraciones de la planta 40 en función del grupo de rigideces adicionales. Comparación de métodos modales: modelo sin reducir, método DACMAM, y condensación DCSS II, caso B (híbrido, con T del SS III).

\begin{tabular}{|l|c|c|c|}
\cline { 2 - 4 } \multicolumn{1}{c|}{ tiempos [s] } & Portátil & Estación 2013 & Estación 2007 \\
\hline Espacio de los estados no simétrico, sin reducc. & 25187 & 16798 & 19026 \\
\hline SS I, sin reducción & 23601 & 16789 & 18897 \\
\hline Método DACMAM con todos los modos & 11465 & 5876 & 8831 \\
\hline DCSS I, caso A & 5879 & 4220 & 6581 \\
\hline DCSS I, caso B (cond. iniciales diferentes) & 5608 & 4171 & 5137 \\
\hline DCSS II, caso A & 5686 & 4190 & 5136 \\
\hline DCSS III & 5705 & 4186 & 5046 \\
\hline DCSS I, caso C (híbrido, con T del SS III) & 5768 & 4185 & 5066 \\
\hline DCSS II, caso B (híbrido, con T del SS III) & 5642 & 4187 & 4921 \\
\hline SCDS, (resuelto en SS I) & 5522 & 4093 & 4292 \\
\hline SCSS I & 5560 & 4094 & 4290 \\
\hline SCSS II & 5514 & 4089 & 4289 \\
\hline
\end{tabular}

Tabla 6.34: Tiempos de computación de los diferentes métodos para el caso de parametrización de rigideces adicionales en las plantas inferiores del edificio. 


\section{Capítulo 7}

\section{CONCLUSIONES}

Ante la necesidad de disponer de métodos de aceleración para los análisis dinámicos sobre grandes estructuras, y que no afecten a la calidad de los resultados, en la tesis se ha realizado una comparación de diferentes métodos de reducción de modelos de análisis estructurales dinámicos y se han aplicado a un caso de estudio sencillo: un edificio de 40 plantas con amortiguamiento no clásico. Se ha presentado la base teórica del método DACMAM (Dynamic Analysis in Complex Modal space Acceleration Method), un método reciente de aceleración de análisis dinámicos para grandes estructuras que proporciona resultados exactos, y se ha demostrado su eficiencia. Se ha comparado tanto la calidad de los resultados obtenidos por este método con los del problema completo y otros métodos de condensación estática y dinámica, como los tiempos de computación que requiere cada uno para la obtención de la respuesta de la estructura del edificio en frecuencias con la presencia de un dispositivo masa-amortiguador sintonizado o tunned mass damper (TMD) en la última planta. En todos los métodos aquí considerados se hace uso de álgebra compleja para obtener la solución. Adicionalmente se han validado los resultados obtenidos con los de la resolución del problema completo integrando numéricamente la ecuación de equilibrio dinámico, empleando para ello un algoritmo Newmark-beta incondicionalmente estable.

También se han comparado los métodos para diversos ejemplos de parametrizaciones estudiando la respuesta de los mismos ante una carga sísmica. Se han validado de nuevo dichos resultados comparándolos con los de un método de integración implícito Newmark-beta con un paso de integración que proporciona resultados correctos. 
Las parametrizaciones consideradas han sido: la parametrización de un TMD para minimizar respuesta en aceleraciones o respuesta en desplazamientos, y la parametrización de las masas no estructurales o de rigideces añadidas para identificar los límites admisibles de ocupación de modo que no los supere la respuesta dinámica de la estructura.

En este trabajo se ha comprobado que el método $D A C M A M$, que se compara con métodos de condensación estáticos clásicos y con métodos de condensación dinámicos iterativos (todos ellos compatibles con amortiguamientos estructurales no clásicos), proporciona ahorros de tiempos de computación importantes y ofrece resultados exactos.

Se ha elegido en la tesis un ejemplo de pequeña dimensión que permite resolver el modelo, con los distintos métodos de reducción mencionados y también sin reducción, en tiempos razonables y cuyos datos son extrapolables a un modelo de grandes dimensiones. Los ahorros de tiempo que se obtienen en el ejemplo son de un orden de magnitud para el método DACMAM frente al modelo completo, y es del mismo orden que el que ofrecen los métodos de reducción con una solución aproximada en lugar de exacta. Este ahorro ya es importante, pero hay que tener en cuenta que para modelos grandes el ahorro es aún más significativo, como muestra el ejemplo de aplicación en Herrada-Martín [2016], donde se obtienen relaciones de 1200 a 1 entre el tiempo de proceso requerido por un código comercial y el empleado por el método DACMAM. Esto pone de relieve que los resultados aquí obtenidos, correctamente extrapolados a un modelo de grandes dimensiones para obtener una estimación realista de los mismos, dan lugar a ahorros de tiempo de varios órdenes de magnitud.

También se ha mostrado en la tesis que los métodos de condensación ofrecen resultados con errores muy bajos para un número reducido de grados de libertad maestros. A medida que dicho número crece, la calidad de los resultados decrece si se limita el número de iteraciones máximo. Visto de otro modo, para mantener la calidad de los resultados es necesario aumentar el número máximo de iteraciones de las condensaciones, lo que implica tiempos de computación mayores, superando aquellos del método DACMAM.

Dicho ahorro de tiempos es especialmente importante para el caso de análisis paramétricos, en los que adicionalmente, según se puede extraer de la metodología del método y del ejemplo de aplicación en Herrada-Martín [2016], los volúmenes de datos que genera son mucho menores, alcanzando una relación de 10000 a 1 en los ficheros de resultados del ejemplo de aplicación. 
Las limitaciones que presenta el método son pequeñas y en todo caso comparables con las del resto de métodos. Sólo cabe destacar que el método no muestra ventajas tan evidentes cuando es necesario supervisar la respuesta dinámica de un número muy elevado de localizaciones de la estructura original.

Se puede concluir, por lo tanto, que el método presentado es particularmente útil para el análisis paramétrico de grandes estructuras con modificaciones locales y discretas, proporcionando ahorros en tiempos de computación de hasta varios órdenes de magnitud en función del tamaño del problema.

$\mathrm{Su}$ implementación con cualquier software comercial de elementos finitos es muy sencilla por sólo requerir de estos la información de los modos propios que se consideren en los puntos de la estructura que se quieran supervisar y la información de los nodos donde se introducen las cargas. Adicionalmente, al poder implementarlo el ingeniero de cálculo gracias a su sencillez, permite realizar las parametrizaciones que sean necesarias de un modo muy simple, tanto las correspondientes a parámetros físicos de la estructura como son masas, rigideces y amortiguamientos, o incluso añadir grados de libertad adicionales, como parametrizar las cargas, densidad de puntos del rango de frecuencias donde se estudia la respuesta, número de modos a tener en consideración a partir de los nodos proporcionados al método, etc.

Durante la tesis se ha realizado la implementación del método de modo que permite realizar: análisis de modos propios, análisis de respuesta en frecuencia, y análisis transitorios con dos variantes: con evaluación de la respuesta temporal, lo que permite la incorporación de los términos transitorios de la respuesta, y empleando la transformada inversa de Fourier, que no tiene en cuenta la parte transitoria de la respuesta pero da tiempos de proceso menores. Atendiendo a las capacidades de parametrización, éstas no están limitadas, ya que al tener un control absoluto de la herramienta se puede adaptar a las necesidades del problema; las opciones ya implementadas corresponden a lo descrito en el método, modificaciones de rigidez, masa, amortiguamiento en cualquier grado de libertad del problema y grados de libertad adicionales. Asimismo, como se puede ver en las figuras de la tesis, el método permite obtener los resultados en gráficas y tablas con control absoluto sobre las variables de interés para el analista, ya sea de los resultados de los mencionados tipos de análisis o de valores máximos, mínimos o dentro de un rango específico. Aunque la implementación en esta tesis no parte de datos de modelos 
de elementos finitos obtenidos de un software comercial, ya se ha comentado que todas estas capacidades son aprovechables de modo muy sencillo para usarse con los resultados de cualquier código comercial, habiendo ya sido probado el método con modelos de los softwares ANSYS y MSC.Nastran.

Como ventajas adicionales también cabe señalar que la base teórica del método DACMAM que aquí se presenta admite el uso de matrices de rigidez y amortiguamiento no simétricas de modo inmediato, sin añadir ninguna complejidad al problema. Esta opción es deseable para estructuras que tengan un control activo o al emplear matrices de amortiguamientos de resultados experimentales.

Por último se debe destacar, que aunque se ha hecho hincapié en la comparación del método $D A C M A M$ con los métodos de condensación descritos, ambos tipos de métodos pueden ser aplicados al mismo tiempo de modo que se obtengan las ventajas de ambos y se eviten sus limitaciones.

\subsection{Otras consideraciones y trabajos futuros}

Durante la realización de esta tesis han surgido varias líneas de investigación posibles relacionadas con la aplicación del método DACMAM que se indican a continuación.

Se ha comentado que el método puede usarse en conjunción con otros métodos de reducción y sería interesante estudiar las posibilidades en este aspecto con métodos de subestructuración, ya que permitiría una reducción adicional en los tiempos de computación proporcionados por aquel en el caso descrito de considerar parametrizaciones y tener interés en su efecto sobre un número reducido de puntos de control.

Una línea de investigación que se ha remarcado en la introducción de la tesis es la aplicación de este método a simulación de daño. El método DACMAM es un candidato óptimo para la generación de resultados necesarios en la implementación y entrenamiento de redes neuronales que requieren una base de datos ingente de resultados muy fiables de comportamiento de la estructura en posiciones discretas, que luego son contrastados con los datos medidos por sensores instalados en la estructura real. La parametrización de la rigidez permite la simulación de diferentes tamaños de daño de manera sencilla, por lo que la validación de los resultados en 
un caso real no requeriría de un esfuerzo elevado en términos de costes de implementación e ingeniería.

Como se ha descrito, las características del método hacen deseable la búsqueda de colaboraciones con empresas que desarrollen modelos de muchos grados de libertad (varios cientos de miles) para continuar con la validación del método en la búsqueda de una respuesta óptima de la estructura ante cargas dinámicas. Un ejemplo podría ser la instalación de TMD individuales en las estructuras de los asientos de vehículos de transporte de personas que ante cargas dinámicas, como puede ser el motor del vehículo, las reacciones del terreno, etc. producen una falta de confort apreciable. El método permitiría, de manera sencilla, la búsqueda del valor óptimo de dichos TMD ante los muy diversos casos de carga variables que soporta la estructura como son la masa de pasajeros, tipos de terrenos por los que circule, frecuencias de vibración del motor, etc.

Aunque en la tesis se ha hablado de las ventajas del método en el caso de parametrizaciones, no se ha profundizado en los métodos para su implementación. En la búsqueda de picos en las respuestas, es sabido que es más eficiente emplear funciones de distribución que asignen valores aleatorios a las variables en lugar del método aquí empleado que ha consistido en valores equiespaciados de los parámetros para un rango dado. Este modo de trabajar es común en el método de Montecarlo y similares, y su implementación influiría positivamente en la calidad de los resultados obtenidos, por lo que se sugiere también seguir la línea de investigación incluyendo dichas técnicas.

En la tesis sólo se ha introducido la capacidad de los métodos para resolver problemas con matrices de rigidez o amortiguamiento no simétricas, pero sería conveniente realizar comparativas adicionales de los métodos aplicados a este tipo de problemas.

Por último, aunque no se ha tratado mucho la aplicación de los resultados de ensayos para refinar las propiedades de los modelos matemáticos que simulan la realidad, este es un campo en el que se continúa investigando actualmente (Jian et al. [2015]) y en el que el método también promete ventajas, pudiendo acelerar los procesos de actualización de modelos a partir de los resultados empíricos. 


\section{Referencias}

Allemang, R. J. y Brown, D. L. 1982. A Correlation Coefficient for modal vector analysis. Pages 110-116 of: $1^{\text {st }}$ International Modal Analysis Conference.

Allemang, R. J. y Brown, D. L. 1987. Experimental Modal Analysis and Dynamic Component Synthesis. Tech. rept. Air Force Wright Aeronautical Laboratories.

ANSYS Mechanical. 2013. ANSYS Mechanical User's Guide 15.0. Ansys, Inc.

Argyris, J. H. y Kelsey, S. 1954,1955. Energy Theorems and Structural Analysis. Aircraft Engineering, 26,27.

Armendáriz, I., Olarrea, J. y García-Martínez, J. 2015. Parametric analysis of a highly dynamical phenomena caused by a propeller blade loss. Engineering Failure Analysis, 57(November), $528-543$.

Aryana, F. y Bahai, H. 2003. Sensitivity analysis and modification of structural dynamic characteristics using second order approximation. Engineering Structures, 25(1279-1287).

Avitabile, P. 2003. Twenty years of Structural Dynamic Modification - A Review. Journal of Sound and Vibration, January, 14-27.

Bahai, H., Farahani, K. y Djoudi, M. S. 2002. Eigenvalue inverse formulation for optimising vibratory behaviour of truss and continuous structures. Computers and Structures, 80(27-30), $2397-2403$.

Baldwind, J. F. y Hutton, S. G. 1985. Natural Modes of Modified Structures. AIAA Journal, 23(11), 1737-1742. 
Basseville, M. 2005 (September 12-14). Lessons Learned from the Theory and practice of Change Detection. En: Proceedings of the $5^{\text {th }}$ International Workshop on Structural Health Monitoring.

Bathe, K. J. 1996. Finite Element Procedures. Prentice Hall.

Besselink, B. 2013. A comparison of model reduction techniques from structural dynamics, numerical mathematics and systems and control. Journal of Sound and Vibration, 332, $4403-4422$.

Bilbao, A., Avilés, R., Agirrebeitia, J. y Ajuria, G. 2006. Proportional damping approximation for structures with added viscoelastic dampers. Finite Elements in Analysis and Design, 42, 492-502.

B.Irons. 1965. Structural eigenvalue problems - elimination of unwanted variables. AIAA Journal, 3(5), 961-962.

Caughey, T. K. 1960. Classical Normal Modes in Damped Linear Dynamic Systems. Journal of Applied Mechanics, 27(2), 269-271.

Caughey, T. K. y O'Kelly, M. E. J. 1965. Classical Normal Modes in Damped Linear Dynamic Systems. Journal of Applied Mechanics, 32, 583-588.

Chen, X., Wang, H. y Ding, Y. 2011. Multi-Domain Parallel Computing for Strength Analysis of Whole Aircraft Model. Journal of Software Engineering and Applications, 4(9), 546-549.

Chopra, A. K. 1995. Dynamic of Structures. Prentice Hall.

Clough, R. W. 1960 (September). The Finite Element Method in Plane Stress Analysis. En: $2^{\text {nd }}$ ASCE Conference On Electronic Computation.

Craig Jr., R. R. y Bampton, M. C. C. 1968. Coupling of Substructures for Dynamic Analyses. AIAA Journal, 6(7), 1313-1319.

Den Hartog, J. P. 1984. Mechanical Vibrations. Dover Publications.

Farrar, C. R., Park, G., Puckett, A. D. y Flynn, E. B. 2006 (October 10-12). Sensing and Sensor Optimization Issues for Structural Health Monitoring. $23^{\text {rd }}$ Aerospace Testing Seminar. 
Flippen Jr., L. D. 1994. A Theory of Condensation Model Reduction. Computers $\& 3$ Mathematics with Applications, 27(2), 9-40.

Friswell, M. I., Garvey, S. D. y Penny, J. E. T. 1995. Model Reduction Using Dynamic and Iterative IRS Techniques. Journal of Sound and Vibration, 2(186), 311-323.

Garatani, K. 1999. Feasibility Study of GeoFEM for Solving 100 millions DOF Problems. Pages 331-336 of: $1^{\text {st }}$ ACES Workshop Proceedings.

García-Hiernaux, A., Casal, J. y Jerez, M. 2007. Detección de raíces unitarias y cointegración mediante métodos de subespacios. Revista Colombiana de Estadística, 30(1), 77-96.

García-Martínez, J., San Millán, F. J., et al. . 2008. FEM simulation of delamination and debonding growth in composite structures. En: International Conference on Simulation Based Engineering and Sciences.

Goldman, R. L. 1969. Vibration analysis by dynamic partitioning. AIAA Journal, 7(6), 11521154.

Golub y van der Vorst. 2000. Eigenvalue computation in the $20^{\text {th }}$ century. Journal of Computational and Applied Mathematics, 123, 35-65.

González, R., Armendáriz, I., García-Martínez-, J. y San Millán, F. J. 2008. Simulación con elementos finitos de propagación de grietas en materiales compuestos. En: XXV Encuentro del Grupo Español de Fractura.

Guyan, R. J. 1965. Reduction of Stiffness and Mass Matrices. AIAA Journal, 3(2), 380.

Harris, Piersol, et al. . 2002. Harris' Shock and Vibration Handbook. McGraw-Hill.

Hays, W. W. 1980. Procedures for Estimating Earthquake Ground Motions. U.S. Geological Survey professional paper; 1114. $77 \mathrm{p}$.

Herrada-Martín, F. J. 2016. Método Computacional para la Aceleración de Análisis Paramétricos de Modificaciones Locales en Estructuras Complejas sometidas a Cargas Dinámicas. Ph.D. thesis, E.T.S.I. Aeronáuticos, U.P.M. 
Hibbit, Karlsson y Sorensen. 2007. Abaqus Analysis User's Manual. Dassault Systemes.

Hou, S. 1969. Review of modal synthesis techniques and a new approach. The Shock and Vibration Bulletin, 40(4), 25-39.

Housner, G. W. 1941. Calculating the response of an oscillator to arbitrary ground motion. Bulletin of the Seismological Society of America, 31(April), 143-149.

Hurty, W. C. 1965. Dynamic Analysis of Structural Systems Using Component Modes. AIAA Journal, 3(4), 678-685.

Jian, H., Sizhu, Z., Lei, H. y Xinmei, Y. 2015. Structural dynamics modification for derrick of deep well drilling rig based on experimental modal test and frequency sensitivity analysis. Advances in Mechanical Engineering, 7(9), 1-7.

Kim, J. y Lee, P. 2014. An Accurate Error Estimator for Guyan Reduction. Computer Methods in Applied Mechanics and Engineering, 278, 1-19.

Kim, K. 1996 (April). Improved Hybrid Dynamic Condensation for Eigenproblems. Pages 756765 of: $37^{\text {th }}$ AIAA/ASME/ASCE/AHS/ASC Structures, Structural Dynamics, and Materials Conference and Exhibit, vol. Technical Papers. AIAA, Salt Lake City, UT.

Lanczos, C. 1950. An iteration method for the solution of the eigenvalue problem of linear differential and integral operators. Journal of Research of the National Bureau of Standards, 45(4), 255-282.

Lázaro, M. y Pérez-Aparicio, J. L. 2013. Multiparametric computation of eigenvalues for linear viscoelastic structures. Computers and Structures, 117, 67-81.

Lázaro, M., Pérez-Aparicio, J. L. y Epstein, M. 2012. Computation of eigenvalues in proportionally damped viscoelastic structures based on the fixed-point iteration. Applied Mathematics and Computation, 219(8), 3511-3529.

Lepidi, M., Gattulli, V. y Vestroni, F. 2007. Static and dynamic response of elastic suspended cables with damage. International Journal of Solids and Structures, 44(25-26), 8194-8212. 
Li, T. y He, J. 1999. Local Structural Modification using mass and stiffness changes. Engineering Structures, 21, 1028-1037.

Liu, Z. y Wu, Z. 2011. Iterative-Order-Reduction Substructuring Method for Dynamic Condensation of Finite Element Models. AIAA Journal, 49(1).

Majkut, L. 2010. Eigenvalue based inverse model of beam for structural modification and diagnosis. Part I: Theoretical formulation. Latin American Journal of Solids and Structures, $7(4), 423-436$.

Martín de La Escalera, F., García-Martínez, J., et al. . 2007. Ensayos de progreso de grieta en probetas de aluminio de espesor delgado, correlación con modelo matemático. En: XXIV Encuentro del Grupo Español de Fractura.

Martínez-Aguirre, M. y Elejabarrieta, M. J. 2011. Higher order eigensensitivities-based numerical method for the harmonic analysis of viscoelastically damped structures. International Journal for Numerical Methods in Engineering, 88(12), 1280-1296.

MATLAB. 2014. Version 6.1 (R2014a). Natick, Massachusetts, United States: The MathWorks Inc.

Montáns, F. J. y Muñoz, I. 2013. Dynamic Analysis of Structures for the Finite Element Method. UNED.

Moore, B. 1981. Principal component analysis in linear systems: Controllability, Observability, and Model Reduction. IEEE Transactions on Automatic Control, 26(1).

Mourelatos, Z. P., Angelis, D. y Skarakis, J. 2012. Advances in Vibration Engineering and Structural Dynamics. InTech, Edited by Francisco Beltran-Carbajal.

MSC.Nastran Ad. Dynamics. 2004. MSC.Nastran Version 70. Advanced Dynamics Analysis Users's Guide. MacNeal-Schwendler Software Corporation.

MSC.Nastran Dynamics. 2014. MSC Nastran 2014 Dynamic Analysis Users's Guide. MacNealSchwendler Software Corporation. 
MSC.Nastran Superelements. 2012. MSC.Nastran Superelements Users's Guide. MacNealSchwendler Software Corporation.

Nad, M. 2007. Structural Dynamic Modification of vibrating systems. Applied Mathematics and Computation, 1, 203-214.

O'Callahan, J. 2003. System Matrix Optimization Using a Complex Analytical Model Improvement (CAMI). En: 21 ${ }^{\text {st }}$ International Modal Analysis Conference.

O'Callahan, J. y Li, P. 1995. The Effect of Modal Vector Expansion on Finite Element Model Updating. University of Massachusetts Lowell, 599-607.

O'Callahan, J., Avitabile, P. y Riemer, R. 1989 (February). System Equivalent Reduction Expansion Process (SEREP). En: $7^{\text {th }}$ International Modal Analysis Conference.

Paz, M. 1984. Dynamic Condensation. AIAA Journal, 22(5), 724-727.

Paz, M. 1989. Modified Dynamic Condensation Method. Journal of Structural Engineering, 115(1), 234-238.

Paz, M. 1992. Dinámica Estructural. Editorial Reverté, S.A.

Pomazal, R. J. y Snyder, V. W. 1971. Local Modifications of Damped Linear Systems. AIAA Journal, 9(11), 2216-2221.

Qu, Z. 2004. Model Order Reduction Techniques. Springer.

Rao. 2002. Dynamic Condensation and Synthesis of Unsymmetric Structural Systems. Journal of Applied Mechanics, 69, 610-616.

Rathinam, M. y Petzold, L. R. 2003. A new look at Proper Orthogonal Decomposition. SIAM Journal on Numerical Analysis, 41(5), 1893-1925.

Rayleigh, J. W. S. 1945. The Theory of Sound. Dover Publications.

Rivera, Singh y Suarez. 1999. Dynamic Condensation Approach for Nonclassically Damped Structures. AIAA Journal, 37, 564-571. 
Rixen, D. J. 2001. Generalized Mode Acceleration Methods and Modal Truncation Augmentation. En: $19^{\text {th }}$ AIAA Applied Aerodynamics Conference, Fluid Dynamics and Co-located Conferences. AIAA 2001, no. 1300.

Rixen, D. J. 2009. Dual Craig-Bampton with Enrichment to Avoid Spurious Modes. En: $27^{\text {th }}$ International Modal Analysis Conference. Society for Experimental Mechanics Inc.

Rouleau, L., Deu, J. L. y Legay, A. 2014. Review of Reduction Methods based on Modal Projection for Highly Damped Structures. En: $11^{\text {th }}$ World Congress on Computational Mechanics.

Schwarz, B. y Richardson, M. 2013. Proportional Damping from Experimental Data. En: $31^{\text {st }}$ International Modal Analysis Conference.

Schwarz, B. J. y Richardson, M. H. 1997. Structural Modifications Using Higher Order Elements. En: $15^{\text {th }}$ International Modal Analysis Conference. Society for Experimental Mechanics Inc.

Schwarz, B. J., Richardson, M. H. y Formenti, D. L. 2007. FEA Model Updating using SDM. En: $25^{\text {th }}$ International Modal Analysis Conference. Society for Experimental Mechanics Inc.

Shabana, A. A. 1996. Theory of Vibration. An Introduction. Springer.

Suarez, L. E. y Singh, M. P. 1992. Dynamic Condensation Method for Structural Eigenvalue Analysis. AIAA Journal, 30(4), 1046-1054.

Sutter, T. R., Camarda, C. J., Walsh, J. L. y Adelman, H. M. 1988. A comparison of several methods for the calculation of vibration mode shape derivatives. AIAA Journal, 26(12), $1506-1511$.

Takeda, N. 2006 (July 5-7). Towards Damage and Structural Health Monitoring of Aerospace Composite Structures using Optical Fiber Sensors. En: Proceedings of the $3^{\text {rd }}$ European Workshop on Structural Health Monitoring,.

Tham, C., Christensen, E. R., Alldredge, D., Hahn, S. y Howsman, T. 2009. The Use of Mode Truncation Augmentation in the Ares I Liftoff Analysis. En: $50^{\text {th }}$ 
AIAA/ASME/ASCE/AHS/ASC Structures, Structural Dynamics, and Materials Conference, Structures, Structural Dynamics, and Materials and Co-located Conferences. AIAA 2009, no. 2679.

Trifunac y Brune. 1970. Complexity of Energy Release during the Imperial Valley, California, Earthquake of 1940. Bulletin of the Seismological Society of America, 60(1), 137-160.

Turner, M. J., Clough, R. W., Martin, H. C. y Topp, L. J. 1956. Stiffness and deflection analysis of complex structures. Journal of the Aeronautical Sciences, 23(9), 805-823.

Udwadia. 2009. A Note on Nonproportional Damping. Journal of Engineering Mechanics, 135(11), 1248-1256.

Valle, M., San Millán, F. J., González, R., García-Martínez, J., et al. . 2008. Simulación del comportamiento en tolerancia al daño de estructuras aeronáuticas. En: XXV Encuentro del Grupo Español de Fractura.

Vibrant Technology. 2014. Using a Tuned Absorber to Suppress Vibration. Tech. rept. Application note 11. Vibrant Technology.

Wallack, P., Skoog, P. y Richardson, M. 1988. Simultaneous Structural Dynamics Modification (S2DM). En: $6^{\text {th }}$ International Modal Analysis Conference. Society for Experimental Mechanics Inc.

Wallack, P., Skoog, P. y Richardson, M. 1989. Comparison of Analytical and Experimental Rib Stiffener Modifications to a Structure. En: $7^{\text {th }}$ International Modal Analysis Conference. Society for Experimental Mechanics Inc.

Williamson, C. y Fixter, L. 2006. State of the Art Review-Structural Health Monitoring. QinetiQ, NAMTEC and the Institute of Materials Minerals and Mining.

Wilson, E. L. 1985. A New Method of Dynamic Analysis for Linear and Nonlinear Systems. Finite Elements for Analysis and Design, 1(1), 21-23.

Wilson, E. L. 2000. Three Dimensional Static and Dynamic Analysis of Structures. Computers and Structures, Inc. 
Wu, J. C., Yang, J. N. y Schmitendorf, W. E. 1998. Reduced-order $\mathrm{H}^{\infty}$ and LQR control for wind-excited tall buildings. Engineering Structures, 20(3), 222-236.

Zeng, Q. 1995. Highly Accurate Modal Method for Calculating Eigenvector Derivatives in Viscous Damping Systems. AIAA Journal, 33(4), 746-751. 
